\title{
THE FERMI LARGE AREA TELESCOPE ON ORBIT: EVENT CLASSIFICATION, INSTRUMENT RESPONSE FUNCTIONS, AND CALIBRATION
}

M. Ackermann ${ }^{1}$, M. Ajello ${ }^{2}$, A. Albert ${ }^{3}$, A. Allafort $^{2}$, W. B. Atwood ${ }^{4}$, M. Axelsson ${ }^{5,6,7}$, L. Baldini $^{8}$, J. Ballet $^{9}$, G. Barbiellini ${ }^{10,11}$, D. Bastieri ${ }^{12,13}$, K. Bechtol ${ }^{2}$, R. Bellazzini ${ }^{14}$, E. Bissaldi ${ }^{15}$, R. D. Blandford ${ }^{2}$, E. D. Bloom ${ }^{2}$, J. R. Bogart ${ }^{2}$, E. Bonamente ${ }^{16,17}$, A. W. Borgland ${ }^{2}$, E. Bottacini ${ }^{2}$, A. Bouvier ${ }^{4}$, T. J. Brandt ${ }^{18}$, J. Bregeon ${ }^{14}$, M. Brigida ${ }^{19,20}$, P. Bruel ${ }^{21}$, R. Buehler ${ }^{2}$, T. H. BurnetT ${ }^{22}$, S. Buson ${ }^{12,13}$, G. A. Caliandro ${ }^{23}$, R. A. Cameron ${ }^{2}$, P. A. Caraveo ${ }^{24}$, J. M. Casandian ${ }^{9}$, E. Cavazzuti ${ }^{25}$, C. Cecchi ${ }^{16,17}$, Ö. Çelik ${ }^{18,26,27}$, E. Charles $^{2}$, R. C. G. Chaves ${ }^{9}$, A. Chekhtman ${ }^{28,67}$, C. C. Cheung ${ }^{29,67,68}$, J. Chiang ${ }^{2}$, S. Ciprini ${ }^{17,25}$, R. Claus ${ }^{2}$, J. Cohen-Tanugi ${ }^{30}$, J. Conrad ${ }^{6,31,69}$, R. Corbet ${ }^{18,27}$, S. Cutini ${ }^{25}$, F. D’Ammando ${ }^{16,32,33}$, D. S. Davis ${ }^{18,27}$, A. De Angelis ${ }^{34}$, M. DeKlotz ${ }^{35}$, F. De Palma ${ }^{19,20}$, C. D. Dermer ${ }^{36}$, S. W. Digel ${ }^{2}$, E. do Couto e Silva ${ }^{2}$, P. S. Drell ${ }^{2}$, A. Drlica-Wagner ${ }^{2}$, R. Dubois ${ }^{2}$, C. Favuzzi ${ }^{19,20}$, S. J. Fegan ${ }^{21}$, E. C. Ferrara ${ }^{18}$, W. B. FocKe ${ }^{2}$, P. Fortin ${ }^{21}$, Y. Fukazawa ${ }^{37}$, S. FunK ${ }^{2}$, P. Fusco ${ }^{19,20}$, F. GarganO ${ }^{20}$, D. Gasparrini ${ }^{25}$, N. Gehrels ${ }^{18}$, B. Giebels ${ }^{21}$, N. Giglietto ${ }^{19,20}$, F. Giordano ${ }^{19,20}$, M. Girolettio ${ }^{38}$, T. GlanZman ${ }^{2}$, G. Godfrey ${ }^{2}$, I. A. Grenier ${ }^{9}$, J. E. Grove ${ }^{36}$, S. Guiriec ${ }^{18}$, D. Hadasch ${ }^{23}$, M. HaYashida ${ }^{2,39}$, E. Hays ${ }^{18}$, D. Horan ${ }^{21}$, X. Hou $^{40}$,

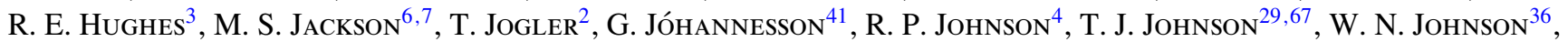

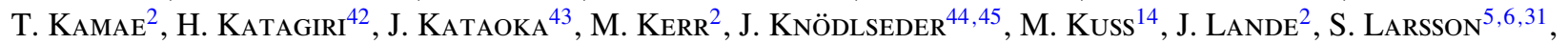
L. Latronico ${ }^{46}$, C. Lavalley ${ }^{30}$, M. Lemoine-Goumard ${ }^{47,70}$, F. Longo ${ }^{10,11}$, F. LoparCo ${ }^{19,20}$, B. LotT $^{47}$, M. N. Lovellette ${ }^{36}$, P. Lubrano ${ }^{16,17}$, M. N. Mazziotta ${ }^{20}$, W. McConville ${ }^{18,48}$, J. E. McEnery ${ }^{18,48}$, J. Mehault ${ }^{30}$, P. F. Michelson ${ }^{2}$, W. Mitthumsiri ${ }^{2}$, T. Mizuno ${ }^{49}$, A. A. Moiseev ${ }^{26,48}$, C. Monte ${ }^{19,20}$, M. E. Monzani ${ }^{2}$, A. Morselli ${ }^{50}$, I. V. Moskalenko ${ }^{2}$, S. Murgia ${ }^{2}$, M. Naumann-Godo ${ }^{9}$, R. Nemmen ${ }^{18}$, S. Nishino ${ }^{37}$, J. P. Norris ${ }^{51}$, E. Nuss ${ }^{30}$, M. Ohno ${ }^{52}$, T. Ohsugi ${ }^{49}$,

A. Okumura ${ }^{2,53}$, N. Omodei ${ }^{2}$, M. Orienti ${ }^{38}$, E. Orlando ${ }^{2}$, J. F. Ormes ${ }^{54}$, D. Paneque ${ }^{2,55}$, J. H. Panetta ${ }^{2}$, J. S. Perkins ${ }^{18,26,27,56}$, M. Pesce-Rollins ${ }^{14}$, M. Pierbattista ${ }^{9}$, F. Piron ${ }^{30}$, G. Pivato ${ }^{13}$, T. A. Porter ${ }^{2}$, J. L. Racusin ${ }^{18}$, S. Rainò ${ }^{19,20}$, R. Rando ${ }^{12,13}$, M. Razzano ${ }^{4,14}$, S. Razzaque ${ }^{28,67}$, A. Reimer ${ }^{2,15}$, O. Reimer ${ }^{2,15}$, T. RePoseur ${ }^{47}$, L. C. Reyes ${ }^{57}$,

S. Ritz ${ }^{4}$, L. S. Rochester ${ }^{2}$, C. Romoli ${ }^{13}$, M. Roth ${ }^{22}$, H. F.-W. SAdrozinski ${ }^{4}$, D. A. SANChEZ ${ }^{58}$, P. M. SAz PARKINSON ${ }^{4}$,

C. Sbarra ${ }^{12}$, J. D. Scargle ${ }^{59}$, C. Sgrò ${ }^{14}$, J. Siegal-Gaskins ${ }^{60}$, E. J. Siskind ${ }^{61}$, G. SPandre ${ }^{14}$, P. Spinellit ${ }^{19,20}$,

T. E. StePhens ${ }^{18,62}$, D. J. SusOn ${ }^{63}$, H. TAJima ${ }^{2,53}$, H. TAKAhashi ${ }^{37}$, T. TANAKA ${ }^{2}$, J. G. ThaYer ${ }^{2}$, J. B. ThaYeR ${ }^{2}$,

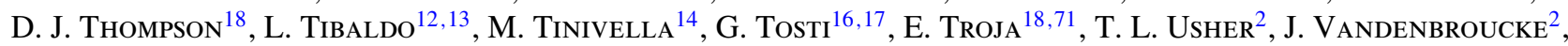
B. Van Klaveren ${ }^{2}$, V. Vasileiou ${ }^{30}$, G. Vianello ${ }^{2,64}$, V. Vitale ${ }^{50,65}$, A. P. Waite ${ }^{2}$, E. Wallace ${ }^{22}$, B. L. Winer ${ }^{3}$,

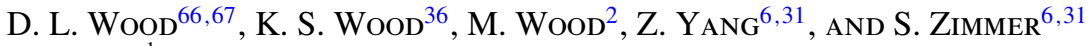

${ }^{1}$ Deutsches Elektronen Synchrotron DESY, D-15738 Zeuthen, Germany

${ }^{2}$ W. W. Hansen Experimental Physics Laboratory, Kavli Institute for Particle Astrophysics and Cosmology,

Department of Physics and SLAC National Accelerator Laboratory, Stanford University, Stanford, CA 94305, USA; echarles@ slac.stanford.edu

${ }^{3}$ Department of Physics, Center for Cosmology and Astro-Particle Physics, The Ohio State University, Columbus, OH 43210, USA

${ }^{4}$ Santa Cruz Institute for Particle Physics, Department of Physics and Department of Astronomy and Astrophysics, University of California at Santa Cruz, Santa Cruz, CA 95064, USA

${ }^{5}$ Department of Astronomy, Stockholm University, SE-106 91 Stockholm, Sweden

${ }^{6}$ The Oskar Klein Centre for Cosmoparticle Physics, AlbaNova, SE-106 91 Stockholm, Sweden

${ }^{7}$ Department of Physics, Royal Institute of Technology (KTH), AlbaNova, SE-106 91 Stockholm, Sweden

${ }^{8}$ Università di Pisa and Istituto Nazionale di Fisica Nucleare, Sezione di Pisa, I-56127 Pisa, Italy; luca.baldini@pi.infn.it

${ }^{9}$ Laboratoire AIM, CEA-IRFU/CNRS/Université Paris Diderot, Service d'Astrophysique, CEA Saclay, F-91191 Gif sur Yvette, France

${ }^{10}$ Istituto Nazionale di Fisica Nucleare, Sezione di Trieste, I-34127 Trieste, Italy

${ }^{11}$ Dipartimento di Fisica, Università di Trieste, I-34127 Trieste, Italy

12 Istituto Nazionale di Fisica Nucleare, Sezione di Padova, I-35131 Padova, Italy; rando@pd.infn.it

${ }^{13}$ Dipartimento di Fisica e Astronomia "G. Galilei," Università di Padova, I-35131 Padova, Italy

${ }^{14}$ Istituto Nazionale di Fisica Nucleare, Sezione di Pisa, I-56127 Pisa, Italy

${ }^{15}$ Institut für Astro- und Teilchenphysik and Institut für Theoretische Physik, Leopold-Franzens-Universität Innsbruck, A-6020 Innsbruck, Austria

${ }^{16}$ Istituto Nazionale di Fisica Nucleare, Sezione di Perugia, I-06123 Perugia, Italy

${ }^{17}$ Dipartimento di Fisica, Università degli Studi di Perugia, I-06123 Perugia, Italy

18 NASA Goddard Space Flight Center, Greenbelt, MD 20771, USA

${ }^{19}$ Dipartimento di Fisica "M. Merlin” dell'Università e del Politecnico di Bari, I-70126 Bari, Italy

${ }^{20}$ Istituto Nazionale di Fisica Nucleare, Sezione di Bari, I-70126 Bari, Italy

${ }^{21}$ Laboratoire Leprince-Ringuet, École polytechnique, CNRS/IN2P3, F-91128 Palaiseau, France

${ }^{22}$ Department of Physics, University of Washington, Seattle, WA 98195-1560, USA

${ }^{23}$ Institut de Ciències de l'Espai (IEEE-CSIC), Campus UAB, E-08193 Barcelona, Spain

${ }^{24}$ INAF-Istituto di Astrofisica Spaziale e Fisica Cosmica, I-20133 Milano, Italy

${ }^{25}$ Agenzia Spaziale Italiana (ASI) Science Data Center, I-00044 Frascati (Roma), Italy

${ }^{26}$ Center for Research and Exploration in Space Science and Technology (CRESST) and NASA Goddard Space Flight Center, Greenbelt, MD 20771, USA

${ }^{27}$ Department of Physics and Center for Space Sciences and Technology, University of Maryland Baltimore County, Baltimore, MD 21250, USA

${ }^{28}$ Center for Earth Observing and Space Research, College of Science, George Mason University, Fairfax, VA 22030, USA

${ }^{29}$ National Academy of Sciences, Washington, DC 20001, USA

${ }^{30}$ Laboratoire Univers et Particules de Montpellier, Université Montpellier 2, CNRS/IN2P3, F-34095 Montpellier, France

${ }^{31}$ Department of Physics, Stockholm University, AlbaNova, SE-106 91 Stockholm, Sweden

${ }^{32}$ IASF Palermo, I-90146 Palermo, Italy

${ }^{33}$ INAF-Istituto di Astrofisica Spaziale e Fisica Cosmica, I-00133 Roma, Italy

${ }^{34}$ Dipartimento di Fisica, Università di Udine and Istituto Nazionale di Fisica Nucleare, Sezione di Trieste, Gruppo Collegato di Udine, I-33100 Udine, Italy 


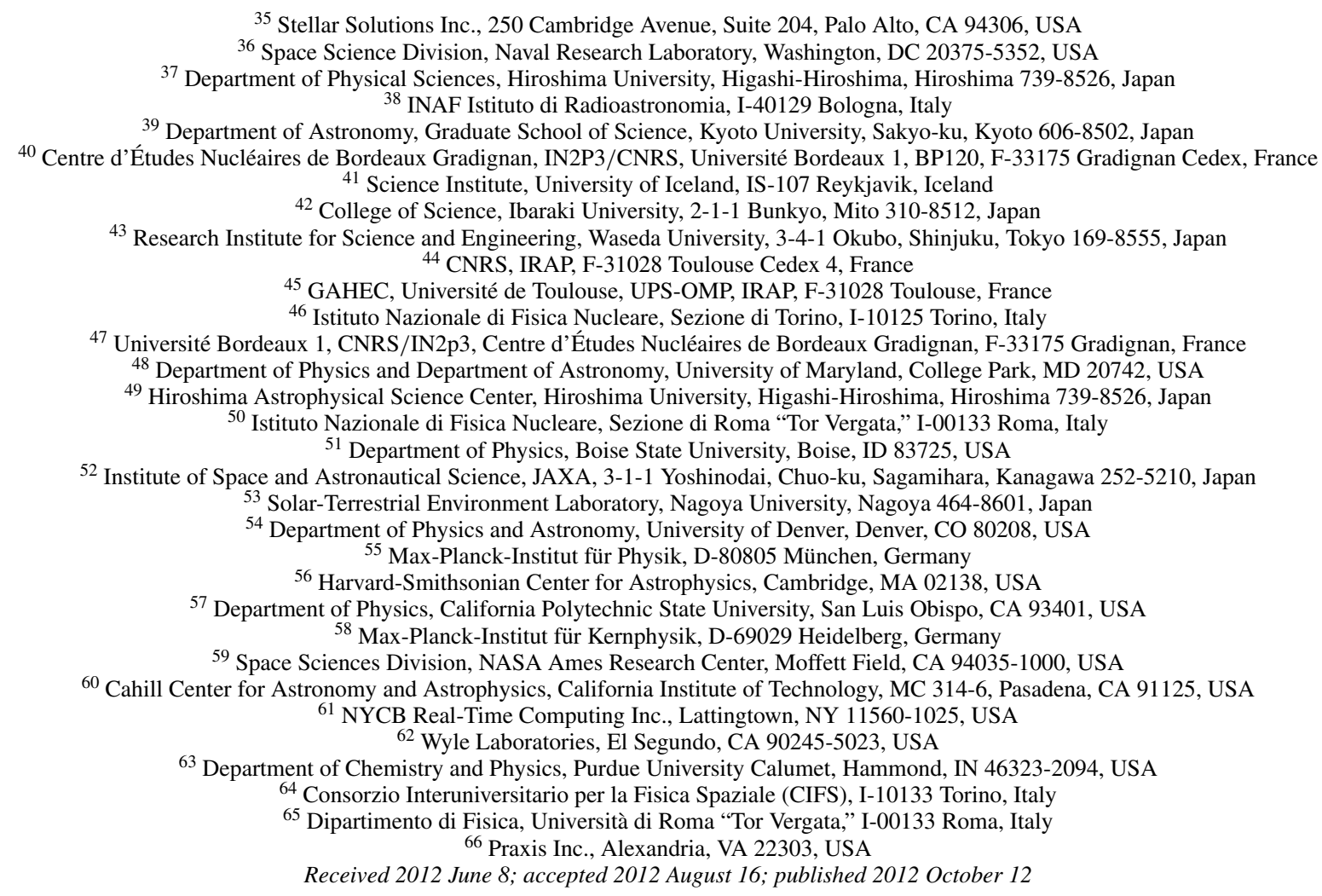

\begin{abstract}
The Fermi Large Area Telescope (Fermi-LAT, hereafter LAT), the primary instrument on the Fermi Gamma-ray Space Telescope (Fermi) mission, is an imaging, wide field-of-view, high-energy $\gamma$-ray telescope, covering the energy range from $20 \mathrm{MeV}$ to more than $300 \mathrm{GeV}$. During the first years of the mission, the LAT team has gained considerable insight into the in-flight performance of the instrument. Accordingly, we have updated the analysis used to reduce LAT data for public release as well as the instrument response functions (IRFs), the description of the instrument performance provided for data analysis. In this paper, we describe the effects that motivated these updates. Furthermore, we discuss how we originally derived IRFs from Monte Carlo simulations and later corrected those IRFs for discrepancies observed between flight and simulated data. We also give details of the validations performed using flight data and quantify the residual uncertainties in the IRFs. Finally, we describe techniques the LAT team has developed to propagate those uncertainties into estimates of the systematic errors on common measurements such as fluxes and spectra of astrophysical sources.
\end{abstract}

Key words: instrumentation: detectors - instrumentation: miscellaneous - methods: data analysis - methods: observational - telescopes

Online-only material: color figures

\section{INTRODUCTION}

The Fermi Gamma-ray Space Telescope (Fermi) was launched on 2008 June 11. Commissioning of the Fermi Large Area Telescope (LAT) began on 2008 June 24 (Abdo et al. 2009a). On 2008 August 4, the LAT began nominal science operations. Approximately one year later the LAT data were publicly released via the Fermi Science Support Center (FSSC). ${ }^{72}$

The LAT is a pair-conversion telescope; individual $\gamma$ rays convert to $e^{+} e^{-}$pairs, which are recorded by the instrument.

\footnotetext{
${ }^{67}$ Resident at Naval Research Laboratory, Washington, DC 20375, USA.

${ }^{68}$ National Research Council Research Associate.

${ }^{69}$ Royal Swedish Academy of Sciences Research Fellow, funded by a grant from the K. A. Wallenberg Foundation.

${ }^{70}$ Funded by contract ERC-StG-259391 from the European Community.

71 NASA Postdoctoral Program Fellow.

72 http://fermi.gsfc.nasa.gov/ssc
}

By reconstructing the $e^{+} e^{-}$pair we can deduce the energy and direction of the incident $\gamma$ ray. Accordingly, LAT data analysis is entirely event-based: we record and analyze each incident particle separately.

In the first three years of LAT observations (from 2008 August 4 to 2011 August 4 ), the LAT read out over $1.8 \times 10^{11}$ individual events, of which $\sim 3.4 \times 10^{10}$ were transmitted to the ground and subsequently analyzed in the LAT data processing pipeline at the LAT Instrument Science Operations Center (ISOC). Of those, $\sim 1.44 \times 10^{8}$ passed detailed $\gamma$-ray selection criteria and entered the LAT public data set.

The LAT team and the FSSC work together to develop, maintain, and publicly distribute a suite of instrument-specific science analysis tools (hereafter ScienceTools ${ }^{73}$ ) that can be used to perform standard astronomical analyses. A critical

\footnotetext{
73 http://fermi.gsfc.nasa.gov/ssc/data/analysis/software
} 
component of these tools is the parameterized representations of instrument performance: the instrument response functions (IRFs). In practice, the LAT team assumes that the IRFs can be factorized into three parts (the validity of this assumption is studied in Section 7.1.4).

1. Effective area, $A_{\mathrm{eff}}(E, \hat{v}, s)$, the product of the crosssectional geometrical collection area, $\gamma$-ray conversion probability, and the efficiency of a given event selection (denoted by $s$ ) for a $\gamma$ ray with energy $E$ and direction $\hat{v}$ in the LAT frame.

2. Point-spread function (PSF), $P\left(\hat{v}^{\prime} ; E, \hat{v}, s\right)$, the probability density to reconstruct an incident direction $\hat{v}^{\prime}$ for a $\gamma$ ray with $(E, \hat{v})$ in the event selection $s$.

3. Energy dispersion, $D\left(E^{\prime} ; E, \hat{v}, s\right)$, the probability density to measure an event energy $E^{\prime}$ for a $\gamma$ ray with $(E, \hat{v})$ in the event selection $s$.

The IRFs described above are designed to be used in a maximum likelihood analysis ${ }^{74}$ as described in Mattox et al. (1996). Given a distribution of $\gamma$ rays $S(E, \hat{p})$, where $\hat{p}$ refers to the celestial directions of the $\gamma$ rays, we can use the IRFs to predict the distribution of observed $\gamma$ rays $M\left(E^{\prime}, \hat{p}^{\prime}, s\right)$ :

$$
\begin{aligned}
& M\left(E^{\prime}, \hat{p}^{\prime}, s\right)=\iiint S(E, \hat{p}) A_{\mathrm{eff}}(E, \hat{v}(t ; \hat{p}), s) \\
& \times P\left(\hat{v}^{\prime}\left(t, \hat{p}^{\prime}\right) ; E, \hat{v}(t ; \hat{p}), s\right) D\left(E^{\prime} ; E, \hat{v}(t ; \hat{p}), s\right) d E d \Omega d t
\end{aligned}
$$

The integrals are over the time range of interest for the analysis, the solid angle in the LAT reference frame and the energy range of the LAT.

Note that the IRFs can change markedly across the LAT field of view (FoV). Therefore, we define the exposure for any given energy and direction in the sky $\mathcal{E}(E, \hat{p})$ as the integral over the time range of interest of the effective area for that particular direction:

$$
\mathcal{E}(E, \hat{p}, s)=\int A_{\mathrm{eff}}(E, \hat{v}(t, \hat{p}), s) d t .
$$

Another important quantity is the distribution of observing time in the LAT reference frame of any given direction in the sky (henceforth referred to as the observing profile, and written $\left.t_{\mathrm{obs}}\right)$, and which is closely related to the exposure:

$$
\mathcal{E}(E, \hat{p}, s)=\int A_{\mathrm{eff}}(E, \hat{v}, s) t_{\mathrm{obs}}(\hat{v} ; \hat{p}) d \Omega .
$$

The absolute timing performance of the LAT has been described in detail in Abdo et al. (2009a) and Smith et al. (2008) and will not be discussed in this paper.

To allow users to perform most standard analyses with minimum effort, the LAT team also provides, via the FSSC, a spatial and spectral model of the Galactic diffuse $\gamma$-ray emission and a spectral template for isotropic $\gamma$-ray emission. ${ }^{75}$ In this prescription, contamination of the $\gamma$-ray sample from residual charged cosmic rays (CRs) is included in the isotropic spectral template. Although not part of the IRFs, this background contamination is an important aspect of the instrument performance.

\footnotetext{
$\overline{74}$ http://fermi.gsfc.nasa.gov/ssc/data/analysis/scitools/ likelihood_tutorial.html

75 http://fermi.gsfc.nasa.gov/ssc/data/access/lat/BackgroundModels.html
}

From the instrument design to the high-level source analysis, the LAT team has relied heavily on Monte Carlo (MC) simulations of $\gamma$-ray interactions with the LAT to characterize performance and develop IRFs. The high-quality data produced since launch have largely validated this choice. However, unsurprisingly, the real flight data exhibited unanticipated features that required modifications to the IRFs. After years of observations, the LAT data set itself is by far the best source of calibration data available to characterize these modifications.

LAT event analysis has substantially improved since launch. We have applied the accumulated updates in occasional rereleases of data, corresponding to reprocessing the entirety of the LAT data to make use of the latest available analysis. In addition to being a resource-consuming task, re-releases require that users download the newly available data and replace all science analysis products. In addition, during the mission, we also made minor improvements in the IRFs based on a better understanding of the properties of the event analysis and an improved description of the LAT performance. These incremental IRFs can be computed and released without modifying existing data, and many of the analysis products remain valid.

We have released two major iterations of the data analysis since launch.

1. Pass 6 indicates the event analysis scheme designed prior to launch. As such, it was based exclusively on our informed estimates of the CR environment at the orbit of Fermi and an MC-based evaluation of the LAT performance. After the commissioning phase, as data started accumulating, we observed phenomena that were not reproduced in the MC simulations (see Sections 2.5 and 5.2). Without modifying the event analysis in any way, we opted to reduce systematic errors by adding these effects to the MC simulations, and we re-evaluated the LAT performance (in particular we calculated new IRFs; see Section 5.2). While this did not allow us to recover any of the lost LAT performance, it ensured that real and simulated data were subject to the same effects and the MC-estimated performance was therefore adequate for science analysis. We have described the initial Pass 6 release (P6_V1) in Atwood et al. (2009), and the corrected IRFs (P6_V3) in Rando \& The Fermi LAT Collaboration (2009). We will discuss some improvements that were incorporated into the later P6_V11 IRFs in Sections 5.4 and 6.2.

2. Pass 7 indicates an improved version of the event analysis, for which we updated parts of the data reduction process to account for known on-orbit effects by making use of the large number of real events the LAT collected in two years of operation. The event reconstruction and the overall analysis design were not modified, but the event classification was re-optimized on simulated data sets including all known on-orbit effects. Large samples of real events were used to assess the efficiency of each step and the systematics involved. Particular attention was paid to increasing the effective area below $\sim 300 \mathrm{MeV}$ where the impact of on-orbit effects was large, while maintaining tolerable rates of $\mathrm{CR}$ contamination at those energies. Event class definitions were optimized based on comparisons of MC events and selected samples of real LAT data. See Section 3 for a description of Pass 7.

All data released prior to 2011 August 1 were based on Pass 6. On 2011 August 1 we released Pass 7 data for 


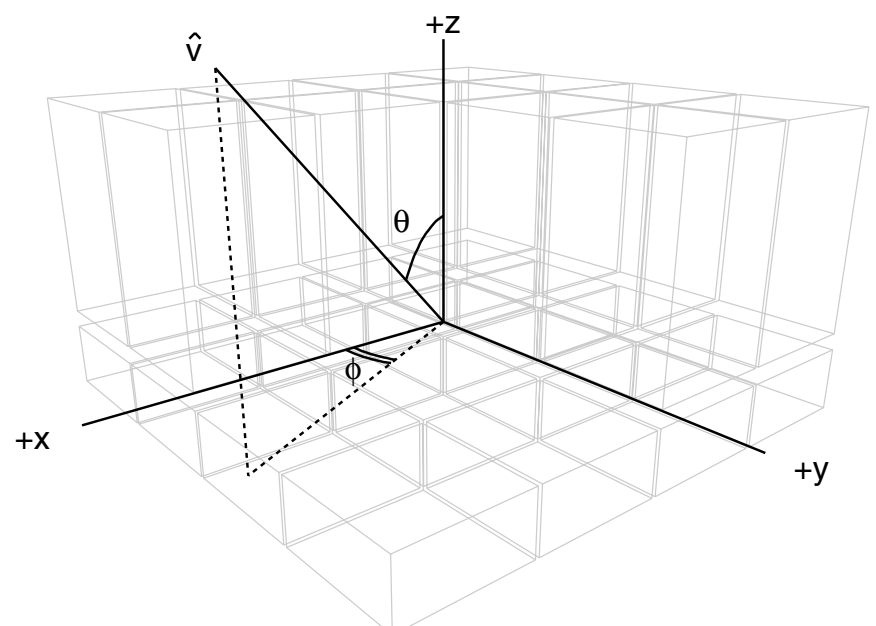

Figure 1. Schematic of the LAT, including the layout of the 16 CAL modules and 12 of the 16 TKR modules (for graphical clarity the ACD is not shown). This figure also defines the $(\theta, \phi)$ coordinate system used throughout the paper.

the entire mission to date, and since then all data have been processed only with Pass 7.

This paper has two primary purposes. The first is to describe Pass 7 (Section 3), quantifying the differences with respect to Pass 6 when necessary. The second is to detail our understanding of the LAT, and toward that end we describe how we have used flight data to validate the generally excellent fidelity of our simulations of particle interactions in the LAT, as well as the resulting IRFs and residual charged particle contamination. In particular, we describe the methods and control data samples we have used to study the residual charged particle contamination (Section 4), effective area (Section 5), PSF (Section 6), and energy dispersion (Section 7) of the LAT. Furthermore, we quantify the uncertainties in each case, and discuss how these uncertainties affect high-level scientific analyses (Section 8).

For convenience, we have included lists of the acronyms and abbreviations (Appendix B) and notation conventions (Appendix C) used in this paper.

\section{LAT INSTRUMENT, ORBITAL ENVIRONMENT, DATA PROCESSING, AND SIMULATIONS}

In this paper, we focus primarily on those aspects of the LAT instrument, data, and analysis algorithms that are most relevant for the understanding and validation of LAT performance. Additional discussion of these subjects was provided in a dedicated paper (Atwood et al. 2009). The calibrations of the LAT subsystems are described in a second paper (Abdo et al. 2009a).

\subsection{LAT Instrument}

The LAT consists of three detector subsystems. A tracker/ converter (TKR), comprising 18 layers of paired $x-y$ silicon strip detector (SSD) planes with interleaved tungsten foils, which promote pair conversion and measure the directions of incident particles (Atwood et al. 2007). A calorimeter (CAL), composed of 8.6 radiation lengths of $\mathrm{CsI}(\mathrm{Tl})$ scintillation crystals stacked in eight layers, provides energy measurements as well as some imaging capability (Grove \& Johnson 2010). An anticoincidence detector (ACD), featuring an array of plastic scintillator tiles and wavelength-shifting fibers, surrounds the TKR and rejects CR backgrounds (Moiseev et al. 2007).

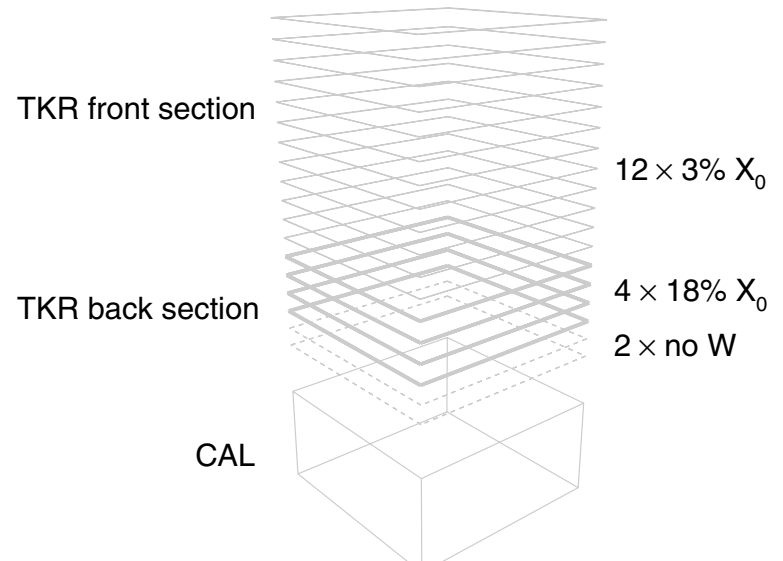

Figure 2. Schematic of a LAT tower (including a TKR and a CAL module). The layout of the tungsten conversion planes in the TKR is illustrated.

In addition to these three subsystems, a triggering and data acquisition system selects and records the most likely $\gamma$-ray candidate events for transmission to the ground. Both the CAL and TKR consist of 16 modules (often referred to as towers) arranged in a $4 \times 4$ grid. Each tower has a footprint of $\sim 37 \mathrm{~cm} \times 37 \mathrm{~cm}$ and is $\sim 85 \mathrm{~cm}$ high (from the top of the TKR to the bottom of the CAL). A schematic of the LAT is shown in Figure 1, and defines the coordinate system used throughout this paper. Note that the $z$-axis corresponds to the LAT boresight, and the incidence $(\theta)$ and azimuth $(\phi)$ angles are defined with respect to the $z$ - and $x$-axes, respectively.

\subsubsection{Silicon Tracker}

The TKR is the section of the LAT where $\gamma$ rays ideally convert to $e^{+} e^{-}$pairs and their trajectories are measured. A full description of the TKR can be found in Atwood et al. (2007, 2009). A simplified schematic of the TKR is shown in Figure 2. Starting from the top (farthest from the CAL), the first 12 paired layers are arranged to immediately follow converter foils, which are composed of $\sim 3 \%$ of a radiation length of tungsten. Minimizing the separation of the converter foils from the following SSD planes, and hence the lever arm between the conversion point and the first position measurements, is critical to minimize the effects of multiple scattering. This section of the TKR is referred to as the thin or front section. The next four layers are similar except that the tungsten converters are $\sim 6$ times thicker; these layers are referred to as the thick or back section. The last two layers have no converter; this is dictated by the TKR trigger, which requires hits in three $x-y$ paired adjacent layers (see Section 3.1.1) and is therefore insensitive to $\gamma$ rays that convert in the last two layers.

Thus, the TKR effectively divides into two distinct instruments with notable differences in performance, especially with respect to the PSF and background contamination. This choice was suggested by the need to balance two basic (and somewhat conflicting) requirements: simultaneously obtaining good angular resolution and a large conversion probability. The tungsten foils were designed such that there are approximately the same number of $\gamma$ rays (integrated over the instrument FoV) converted in the thin and thick sections. In addition to these considerations, experience on-orbit has also revealed that the aggregate of the thick layers ( $\sim 0.8$ radiation lengths) limits the amount of backscattered particles from the CAL returning into the TKR and $\mathrm{ACD}$ in high-energy events (i.e., the CAL backsplash) and reduces tails of showers in the TKR from events entering the back 

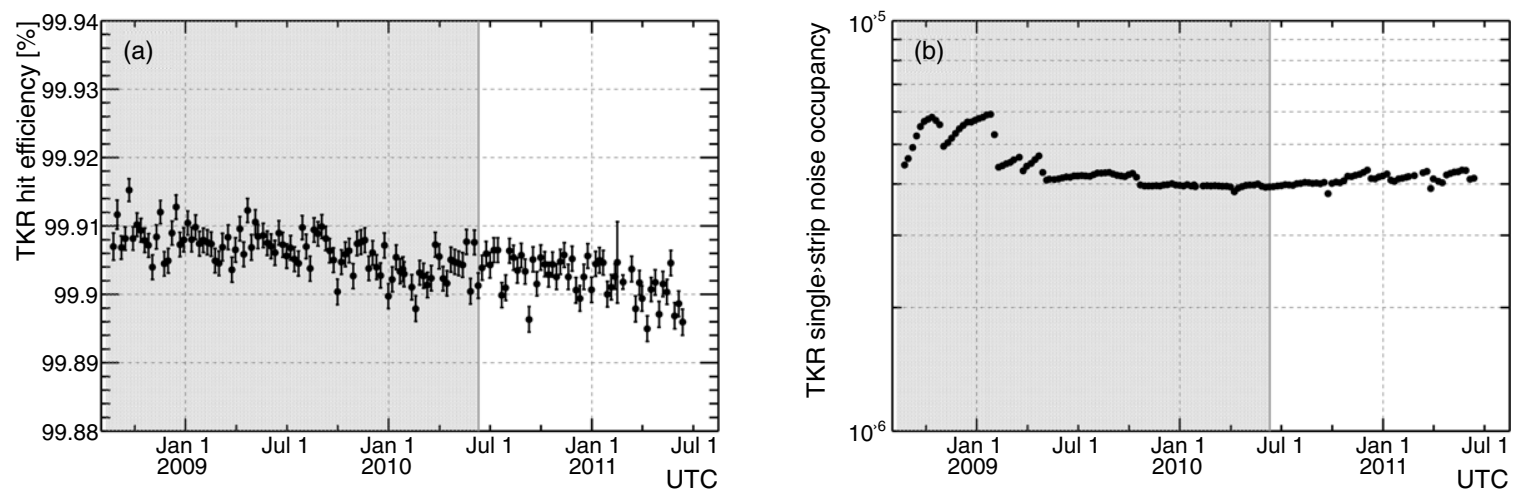

Figure 3. (a) Average TKR hit efficiency and (b) single-strip noise occupancy through the first three years of the mission. Each data point is the average value for the full LAT over a week of data taking. The shaded background regions mark the first two years of operation, corresponding to the data selection used to calibrate the instrument performance.

of the CAL. These two effects help to decrease the background contamination in front-converting events.

After three years of on-orbit experience with the TKR we can now assess the validity of our design decisions. The choice of the solid-state TKR technology has resulted in negligible down time and extremely stable operation, minimizing the necessity for calibrations. Furthermore, the very high signal-to-noise ratio of the TKR analog readout electronics has resulted in a single hit efficiency, averaged over the active silicon surface, greater than $99.8 \%$, with a typical noise occupancy smaller than $10^{-5}$ for a single readout channel. (We note for completeness that the fraction of non-active area presented by the TKR is $\sim 11 \%$ at normal incidence.) As discussed below, this has yielded extremely high efficiency for finding tracks and has been key to providing the information necessary to reject backgrounds.

The efficiency and noise occupancy of the TKR over the first three years of operation are shown in Figure 3. The variations in the average single-strip noise occupancy are dominated by one or a few noisy strips, which have been disabled at different times during the mission. The baseline of $4 \times 10^{-6}$ is dominated by accidental coincidences between event readouts and charged particle tracks (see below and Section 2.1.4) and corresponds to an upper limit of $\sim 3$ noise hits per event in the full LAT on average. Since these noise hits are distributed across 16 towers and 36 layers per tower, their effect on the event reconstruction is insignificant.

The TKR readout is digital, i.e., the readout is binary, with a single threshold discriminator for each channel, and no pulse height information is collected at the strip level. The individual electronic chains connected to each SSD strip consist of a charge-sensitive preamplifier followed by a simple CR-RC shaper with a peaking time of $\sim 1.5 \mu \mathrm{s}$. Due to the implementation details, the baseline restoration tends to be current-limited, and the signal at the output of the shaper is far from the exponential decay characteristic of a linear network, with the discriminated output being high for $\sim 10 \mu \mathrm{s}$ for minimum ionizing particles (MIPs) at the nominal $\sim 1 / 4$ MIP threshold setting. As a consequence, the latched TKR strip signals are typically present for that amount of time after the passage of an MIP. If the LAT is triggered within this time window, these latent signals will be read out and become part of the TKR event data. The rate of occurrence of these ghost signals (which may result in additional tracks when the events are reconstructed) depends directly on the charged particle background rate. Mitigation against this contamination in the data is discussed in Sections 2.1.4, 3, and 5.2.

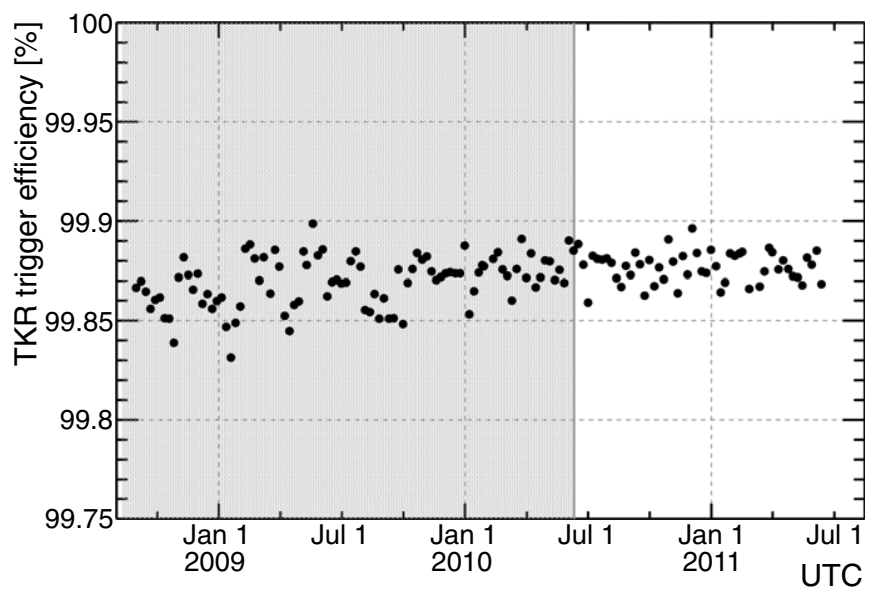

Figure 4. Average efficiency of the TKR three-in-a-row trigger through the first three years of the mission. Each data point is the average value for the full LAT over a week of data taking. The shaded background region marks the first two years of operation, corresponding to the data selection used to calibrate the instrument performance.

Each detector subsystem contributes one or more trigger primitive signals that the LAT trigger system uses to adjudicate whether to read out the LAT detectors (see Section 3.1). The TKR trigger is a coincidence of three $x-y$ paired adjacent layers within a single tower (hence a total of six consecutive SSD detector planes). Due primarily to power constraints, the TKR electronic system does not feature a dedicated fast signal for trigger purposes. Rather, the logical OR of the discriminated strip signals from each detector plane is used to initiate a nonretriggerable one-shot pulse of fixed length (typically 32 clock ticks or $1.6 \mu \mathrm{s}$ ) that is the basic building block of the three-in-arow trigger primitive (see Section 3.1.1). In addition, the length (or time over threshold) of this layer-OR signal is recorded and included in the data stream. Since the time over threshold depends on the magnitude of the ionization in the SSD, which in turn depends on the characteristics of the ionizing particle, it provides useful information for the background rejection stage.

The efficiency of the three-in-a-row trigger is $>99 \%$. This is due in part to the redundancy of this trigger for the vast majority of events (i.e., by passing through many layers of $\mathrm{Si}$, most events have multiple opportunities to form a three-in-arow). The trigger efficiency, measured in flight using MIP tracks crossing multiple towers, is shown to be very stable in Figure 4. 
Perhaps the most important figure of merit for the TKR is the resulting PSF for reconstructed $\gamma$-ray directions. At low energy, the PSF is dominated by multiple scattering, primarily within the tungsten conversion foils (tungsten accounts for $\sim 67 \%$ of the material in the thin section and $\sim 92 \%$ of the material in the thick section). At high energy, the combination of the strip pitch of $228 \mu \mathrm{m}$, the spacing of the planes, and the overall height of the TKR result in a limiting precision for the average conversion of $\sim 0$. 1 at normal incidence. MC simulations predict that the transition to this measurement-precision-dominated regime should occur between $\sim 3 \mathrm{GeV}$ and $\sim 20 \mathrm{GeV}$. We will discuss the PSF in significantly more detail in Section 6.

\subsubsection{Electromagnetic Calorimeter}

Details of the CAL can be found in Atwood et al. (2009) and Grove \& Johnson (2010). Here, we highlight some key aspects for understanding the LAT performance. The CAL is a three-dimensional (3D) imaging calorimeter. This is achieved by arranging the CsI crystals in each tower module in 8 layers, each with 12 crystal $\log s$ (with dimensions $326 \mathrm{~mm} \times 26.7 \mathrm{~mm} \times$ $19.9 \mathrm{~mm}$ ) spanning the width of the layer. The logs of alternating layers are rotated by $90^{\circ}$ about the LAT boresight, and aligned with the $x$ - and $y$-axes of the LAT coordinate system.

Each $\log$ is read out with four photodiodes, two at each end: a large photodiode covering low energies $(<1 \mathrm{GeV}$ per crystal), and a small photodiode covering high energies $(<70 \mathrm{GeV}$ per crystal). Each photodiode is connected to a charge-sensitive preamplifier whose output drives (1) a slow $(\sim 3.5 \mu$ s peaking time) shaping amplifier for spectroscopy and (2) a fast shaping amplifier $(\sim 0.5 \mu$ s peaking time $)$ for trigger discrimination. In addition, the output of each slow shaper is connected to two separate track-and-hold stages with different gains $(\times 1$ and $\times 8)$.

The outputs of the four track-and-hold stages are multiplexed to a single analog-to-digital converter. The four gain ranges (two photodiodes $\times$ two track-and-hold gains) span an enormous dynamic range, from $<2 \mathrm{MeV}$ to $70 \mathrm{GeV}$ deposited per crystal, which is necessary to cover the full energy range of the LAT. A zero-suppression discriminator on each crystal reduces the CAL data by eliminating the signals from all crystals with energies $<2 \mathrm{MeV}$. To minimize CAL data volume, each log end reports only a single range, the lowest gain unsaturated range (the onerange, best-range readout) for most events. For likely heavy ions, each $\log$ end reports all four ranges (the four-range readout) for calibrating the energy scale across the different ranges (see Section 3.1.1 for details of how the readout mode is selected).

The CAL provides two inputs to the global LAT trigger. At each log end the output of each fast shaper (for both the large and the small diode) is compared to an adjustable threshold by a discriminator to form two separate trigger request signals. In the standard science configuration, the discriminator thresholds are set at $100 \mathrm{MeV}$ and $1 \mathrm{GeV}$ energy deposition. The $1 \mathrm{GeV}$ threshold is $>90 \%$ efficient for incident $\gamma$ rays above $20 \mathrm{GeV}$.

For each log with deposited energy, two position coordinates are derived simply from the geometrical location of the log within the CAL array, while the longitudinal position is derived from the ratio of signals at opposite ends of the log: the crystal surfaces were treated to provide monotonically decreasing scintillation light collection with increasing distance from a photodiode. Thus, the CAL provides a 3D image of the energy deposition for each event.

Since the CAL is only 8.6 radiation lengths thick at normal incidence, for energies greater than a few GeV shower leakage becomes the dominant factor limiting the energy resolution, in particular because event-to-event variations in the early shower development cause fluctuations in the leakage out the back of the CAL. Indeed, by $\sim 100 \mathrm{GeV}$ about half of the total energy in showers at normal incidence escapes out the back of the LAT on average. The intrinsic 3D imaging capability of the CAL is key to mitigating the degradation of the energy resolution at high energy through an event-by-event 3D fit to the shower profile. This was demonstrated both in beam tests and in simulations (Baldini et al. 2007; Ackermann et al. 2010), achieving better than $10 \%$ energy resolution well past $100 \mathrm{GeV}$. The imaging capability also plays a critical role in the rejection of hadronic showers (see Section 3.3.7). Furthermore, for events depositing more than $\sim 1 \mathrm{GeV}$ in the CAL, imaging in the CAL can be exploited to aid in the TKR reconstruction and in determining the event direction (see Section 3.2).

We have monitored the performance of the CAL continuously over its three years of operation. We observe a slow $(\sim 1 \%$ per year) decrease in the scintillation light yield of the crystals due to radiation damage in low Earth orbit, as we anticipated prior to launch (see also Section 7.3.2). Although we do not yet correct for this decreased light yield, we have derived calibration constants on a channel-by-channel basis for the mission to date. In 2012 January we started reprocessing the full data set with these updated calibrations, and in the future expect to maintain a quarterly cadence of updates to ensure that the calibrated values do not drift by more than $0.5 \%$.

\subsubsection{Anticoincidence Detector}

The third LAT subsystem is the ACD, critically important for the identification of LAT-entering charged CRs. Details of its design can be found in Moiseev et al. (2007) and Atwood et al. (2009). From the experience of the LAT predecessor, the Energetic Gamma-Ray Experiment Telescope (EGRET), came the realization that a high degree of segmentation was required in order to minimize self-veto due to hard X-ray back-scattering (often referred to as backsplash) from showers in the CAL (Esposito et al. 1999).

The ACD consists of 25 scintillating plastic tiles covering the top of the instrument and 16 tiles covering each of the four sides ( 89 in all). The dimensions of the tiles range between 561 and $2650 \mathrm{~cm}^{2}$ in geometrical surface and between 10 and $12 \mathrm{~mm}$ in thickness. By design, the segmentation of the ACD does not match that of the LAT tower modules, to avoid lining up gaps between tiles with gaps in the TKR and CAL. The design requirements for the $\mathrm{ACD}$ specified the capability to reject entering charged particles with an efficiency $>99.97 \%$. To meet the efficiency requirement, careful design of light collection using wavelength-shifting fibers, and meticulous care in the fabrication to maintain the maximum light yield were needed. The result was an average light yield of $\sim 23$ photoelectrons (p.e.) for a normally incident MIP for each of the two redundant readouts for each tile.

The required segmentation inevitably led to less than complete hermeticity, with construction and launch survival considerations setting lower limits on the sizes of the gaps between tiles. Tiles overlap in one dimension, leaving gaps between tile rows in the other. The gaps are $\sim 2.5 \mathrm{~mm}$ and coverage of these gaps is provided by bundles of scintillating fibers (called ribbons), read out at each end by a photomultiplier tube (PMT). The light yield for these ribbons, however, is considerably less than for the tiles: it is typically $\sim 8$ p.e./MIP and varies considerably along the length of a bundle. Therefore, along the gaps the efficiency for detecting the passage of charged particles is 


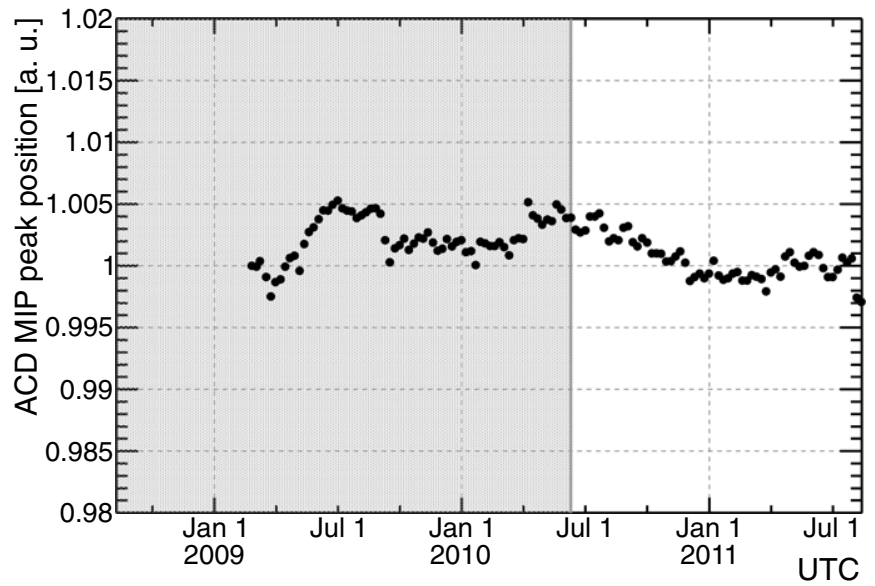

Figure 5. Relative position of the MIP peak, calibrated for the tile response and corrected for the incidence angle, through the first three years of the mission. Each data point is the average value for the 89 ACD tiles over a week of data taking, with the value of the first point being arbitrarily set to 1 . The shaded background region marks the first two years of operation, corresponding to the data selection used to calibrate the instrument performance. Data from the first several months of the mission are missing because this quantity had not yet been included as part of the automated data monitoring and trending.

lower. However, the gaps comprise a small fraction of the total area $(<1 \%)$ and accommodating them did not require compromising the design requirements. In addition to the gaps between ACD tile rows, the corners on the sides of the ACD have gaps that are not covered by ribbons and must be accounted for in the reconstruction and event classification (see Section 3.4).

As with the TKR and CAL, the ACD provides information used in the hardware trigger as well as in the full reconstruction of the event. The output of each PMT is connected to (1) a fast shaping amplifier (with $\sim 400 \mathrm{~ns}$ shaping time) for trigger purposes and (2) two separate slow electronics chains (with $\sim 4 \mu$ s shaping time and different gains) to measure the signal amplitude. The use of the fast signal in the context of the LAT global trigger will be discussed in more detail in Section 3. Although the main purpose of the ACD is to signal the passage of charged particles, this subsystem also provides pulse height information. The two independent slow signals and the accompanying circuitry for automatic range selection accommodate a large dynamic range, from $\sim 0.1$ MIP to hundreds of MIPs.

As for both the TKR and the CAL, the performance of the ACD has been continuously monitored over the past three years. The stability of the MIP signal is shown in Figure 5 and in summary shows very little degradation. Note that slight changes in the selection criteria and spectra of the MIP calibration event sample cause small $(<0.5 \%)$ variations in mean deposited energy per event.

\subsubsection{Trigger and Data Acquisition}

The LAT hardware trigger collects information from the LAT subsystems and, if certain conditions are fulfilled, initiates event readout. Because each readout cycle produces a minimum of $26.5 \mu \mathrm{s}$ of dead time (even more if the readout buffers fill dynamically), the trigger was designed to be efficient for $\gamma$ rays while keeping the total trigger rate, which is dominated by charged CRs, low enough to limit the dead-time fraction to less than about $10 \%$ (which corresponds to a readout rate of about $3.8 \mathrm{kHz}$ ). The triggering criteria are programmable to allow additional, prescaled event streams for continuous in-
Table 1

Characteristic Readout Time Windows for LAT Subsystems

\begin{tabular}{lcc}
\hline \hline Subsystem & $\begin{array}{c}\text { Fast Signal } \\
\text { (Trigger) }\end{array}$ & $\begin{array}{c}\text { Slow Signal } \\
\text { (Event Data) }\end{array}$ \\
\hline ACD & $0.4 \mu \mathrm{s}$ & $4 \mu \mathrm{s}$ \\
CAL & $0.5 \mu \mathrm{s}$ & $3.5 \mu \mathrm{s}$ \\
TKR & $1.5 \mu \mathrm{s}$ & $10 \mu \mathrm{s}$ \\
\hline
\end{tabular}

Notes. The TKR subsystem does not provide a dedicated fast signal for the trigger: the peaking time for the shaped TKR signal is $\sim 1.5 \mu$ s (which is the relevant number for the trigger) but the decay time is much longer and the average time over threshold for the discriminated output is of the order of $10 \mu$ s for an MIP at normal incidence.

strument monitoring and calibration during normal operation. We will defer discussion of the actual configuration used in standard science operations and of the corresponding performance to the more general discussion of event processing in Section 3.1.

To limit the data volume to the available telemetry bandwidth, collected data are passed to the on-board filter. The on-board filter (see Section 3.1.2) consists of a few event selection algorithms running in parallel, each independently able to accept a given event for inclusion in the data stream to be downlinked. Buffers on the input to the on-board filter can store on the order of 100 events awaiting processing. Provided that the onboard filter processes at least the average incoming data rate no additional dead time will be accrued because the on-board filter is busy. The processors used for the on-board filter must also build and compress the events for output, and the time required to make a filter decision varies widely between events. Therefore, the event rate that the on-board filter can handle depends on the number of events passing the filter. In broad terms, the processors will saturate for output rates between $1 \mathrm{kHz}$ and $2.5 \mathrm{kHz}$, depending on the configuration of the on-board filter. In practice, the average output rate is about $350 \mathrm{~Hz}$, and the amount of dead time introduced by the on-board filter is negligible.

Soon after launch, it became apparent that the LAT was recording events that included an unanticipated background: remnants of electronic signals from particles that traversed the LAT a few $\mu$ s before the particle that triggered the event. We refer to these remnants as ghosts. An example of such an event is shown in Figure 6.

It is important to re-emphasize a point made in the previous subsections: many of the signals that are passed to the hardware trigger and the on-board filter are generated by dedicated circuits whose shaping times are significantly shorter than for the circuits that read out the data from the same sensors for transmission to the ground. Although the signals with longer shaping times used for the ground event processing measure the energy deposited in the individual channels far more precisely, they also suffer the adverse consequence of being more susceptible to ghost particles. Table 1 gives the characteristic times for both the fast signals (i.e., those used in the trigger) and the slow signals (i.e., those used in the event-level analysis) for each of the subsystems.

The ghost events have been the largest detrimental effect observed in flight data. They affect all of the subsystems, significantly complicate the event reconstruction and analysis, and can cause serious errors in event classification. 


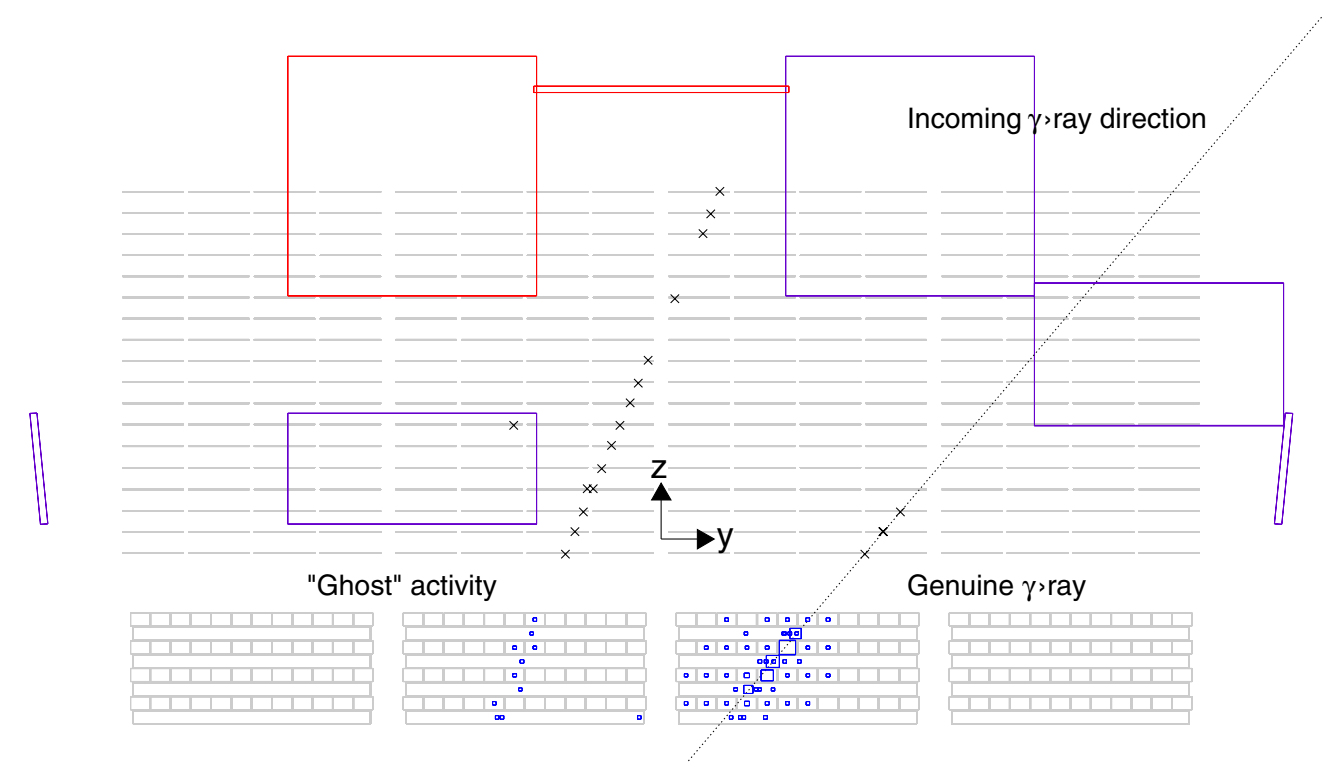

Figure 6. Example of a ghost event in the LAT ( $y-z$ orthogonal projection). In addition to an $8.5 \mathrm{GeV}$ back-converting $\gamma$-ray candidate (on the right) there is additional activity in all three LAT subsystems, with the remnants of a charged particle track crossing the ACD, TKR, and CAL. The small crosses represent the clusters (i.e., groups of adjacent hit strips) in the TKR, while the variable-size squares indicate the reconstructed location of the energy deposition for every hit crystal in the CAL (the side of the square being proportional to the magnitude of the energy release). The dashed line indicates the $\gamma$-ray direction. For graphical clarity, only the ACD volumes with a signal above the zero suppression level are displayed.

(A color version of this figure is available in the online journal.)

1. They can leave spurious tracks in the TKR that do not point in the same direction as the incident $\gamma$ ray and might not be associated with the correct ACD and CAL information due to the different time constants of the subsystems.

2. They can leave sizable signals in the CAL that can confuse the reconstruction of the electromagnetic shower, degrading the measurement of the shape and direction of the shower itself, and that can cause the energy reconstruction to misestimate the energy of the incident $\gamma$ ray.

3. They can leave significant signals in the ACD that can be much larger than ordinary backsplash from the CAL and that can cause an otherwise reconstructable $\gamma$ ray to be classified as a CR.

We will discuss the effects on the LAT effective area in more detail in Section 5. In brief, above $\sim 1 \mathrm{GeV}$, where the average fractional ghost signal in the CAL is small, the loss of effective area is of the order of $10 \%$ or less. This loss is largely due to the fact that valid $\gamma$ rays can be rejected in event reconstruction and classification if ghost energy depositions in the CAL cause a disagreement between the apparent shower directions in CAL and TKR. At lower $\gamma$-ray energies ghost signals can represent the dominant contribution to the energy deposition in the instrument, and the corresponding loss of the effective area can exceed $20 \%$. We also emphasize that, while these figures are averaged over orbital conditions, the fraction of events that suffer from ghost signals, as well as the quantity of ghost signals present in the affected events, varies by a factor of 2-3 at different points in the orbit due to variation in the geomagnetic cutoff and resulting CR rates. Recovering the effective area loss due to ghost signals is one of the original and main motivations of ongoing improvements to the reconstruction (Rochester et al. 2010).

Finally, during extremely bright solar flares (SFs) the pileup of several $>10 \mathrm{keV}$ X-rays within a time interval comparable to the ACD signal shaping time can also cause $\gamma$ rays to be classified as CRs (see Appendix A).

\subsection{Orbital Environment and Event Rates}

Fermi is in a $565 \mathrm{~km}$ altitude orbit with an inclination of $25^{\circ} .6$. The orbit has a period of $\sim 96$ minutes, and its pole precesses about the celestial pole with a period of $\sim 53.4$ days. At this inclination, Fermi spends about $15 \%$ of the time inside the South Atlantic Anomaly (SAA). Science data taking is suspended while Fermi is within the SAA because of the high flux of trapped particles (for details see Abdo et al. 2009a).

When Fermi is in standard sky-survey mode, the spacecraft rocks north and south about the orbital plane on alternate orbits. Specifically, the LAT boresight is offset from the zenith toward either the north or south orbital poles by a characteristic rocking angle. On 2009 September 3, this rocking angle was increased from $35^{\circ}$ to $50^{\circ}$ in order to lower the temperature of the spacecraft batteries and thus extend their lifetime. As a result of this change, the amount of the Earth limb that is subtended by the FoV of the LAT during survey-mode observations increased substantially. The most noticeable consequence is a much larger contribution to the LAT data volume from atmospheric $\gamma$ rays. This will be discussed more in Section 4, and further details about atmospheric $\gamma$ rays can be found in Abdo et al. (2009c).

The flux of charged particles passing through the LAT is usually several thousand times larger than the $\gamma$-ray flux. Accordingly, several stages of event selection are needed to purify the $\gamma$-ray content (see Sections 3.1, 2.4, and 3) and some care must be taken to account for contamination of the $\gamma$-ray sample by charged particles that are incorrectly classified as $\gamma$ rays (see Section 4). Here we distinguish four stages of the event classification process.

1. Hardware trigger request. The LAT detects some traces of particle interaction and starts the triggering process (Section 3.1).

2. Hardware trigger accept. The event in question generates an acceptable trigger pattern and is read out and passed to the on-board filter (Section 3.1). 


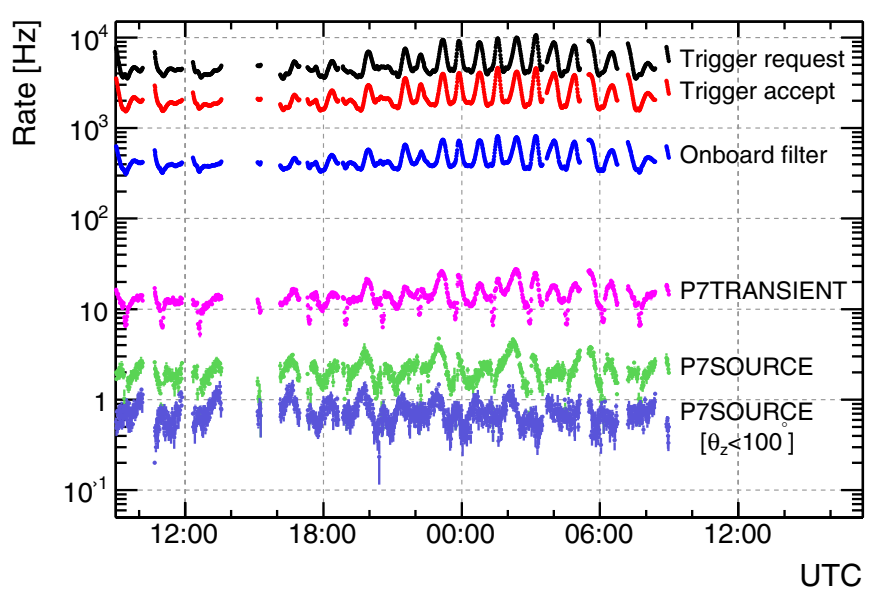

Figure 7. Rates at several stages of the data acquisition and reduction process on a typical day (2011 August 17). Starting from the highest, the curves shown are for the rates (1) at the input of the hardware trigger process (trigger request), (2) at the output of the hardware trigger (trigger accept), (3) at the output of the on-board filter, (4) after the loose P7TRANSIENT $\gamma$-ray selection, (5) after the tighter P7SOURCE $\gamma$-ray selection, and (6) the P7SOURCE $\gamma$-ray selection with an additional cut on the zenith angle $\left(\theta_{z}<100^{\circ}\right)$. See Section 3 for more details about the event selection stages.

(A color version of this figure is available in the online journal.)

3. On-board filter. The event passes the on-board $\gamma$-ray filter criteria (Section 3.1).

4. Standard $\gamma$-ray selection. The event passes more stringent selection criteria (Section 3.4), such as P7SOURCE, which is the selection currently being recommended for analysis of individual point sources, or P6_DIFFUSE, the selection recommended for analysis of point sources in the Pass 6 iteration of the event selections.

Figure 7 shows an example of both the orbital variations of the charged particle flux, and how the initially overwhelming CR contamination is made tractable while maintaining high efficiency for $\gamma$ rays by several stages of data reduction and analysis. Figure 8 shows how the charged particle rate varies with spacecraft position. In particular, data taken during the parts of the orbit with the highest background rates are more difficult to analyze for two reasons: (1) there are simply more non- $\gamma$-ray events that must be rejected and (2) the increased ghost signals complicate the analysis of the $\gamma$-ray events (see Section 2.5.1).

A model of the particle backgrounds for the region outside the SAA was compiled before launch as documented in Atwood et al. (2009): above the geomagnetic cutoff rigidity ( $\sim 10 \mathrm{GV})$ the most abundant species is primary galactic CR protons; at lower energies particle fluxes are dominated by trapped protons and electrons, in addition to the $\gamma$ rays due to interaction of CRs with the atmosphere of the Earth. Since launch, the particle model has been updated to include new measurements (see, e.g., Ackermann et al. 2010, for the electron population).

\subsection{Observing Strategy and Paths of Sources across the LAT Field of View}

Fermi has spent over $95 \%$ of the mission in sky-survey mode, with most of the remainder split between pointed observations and calibrations. Furthermore, in sky-survey mode, the azimuthal orientation of the LAT is constrained by the need to keep the spacecraft solar panels pointed toward the Sun and the radiators away from the Sun. Specifically, in sky-survey mode Fermi completes one full rotation in $\phi$ every orbit.

Therefore, during an orbital precession period the LAT boresight will cross a range of declinations \pm 25.6 relative to the rocking angle, while the LAT $x$-axis will track the direction toward the Sun. As a result, during the sky-survey mode, each point in the sky traces a complicated path across the LAT FoV, the details of which depend on the declination and ecliptic latitude. Figure 9 shows examples of these paths for two of the brightest LAT sources. Furthermore, in sky-survey mode operations the path that the LAT boresight traces across the sky during any two orbit period is only slightly different than during the two previous or subsequent orbits.

\subsection{Ground-based Data Processing}

Reconstructing the signals in the individual detector channels into a coherent picture of a particle interaction with the LAT for each of the several hundred events collected every second is a formidable task. We will defer detailed discussion of the event reconstruction and classification to Section 3; here we describe just the steps to give a sense of the constraints.
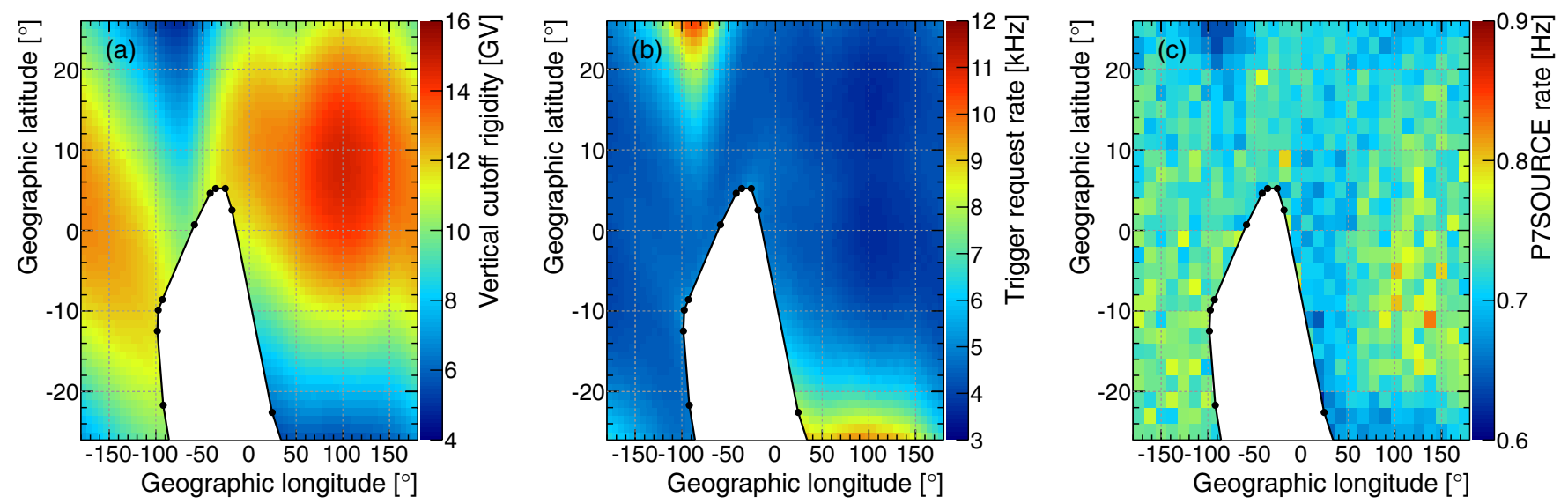

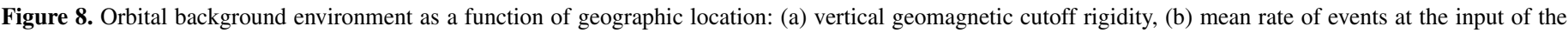

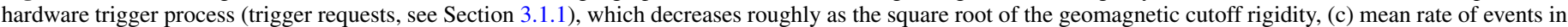

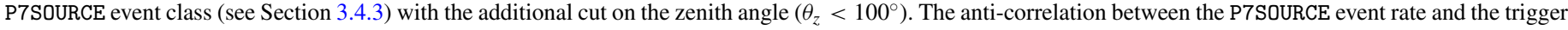

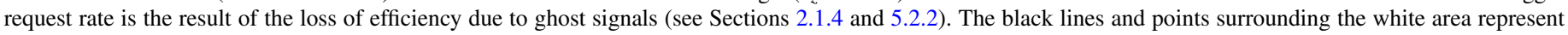
the sides and vertices of the SAA polygon as defined for LAT operations. The LAT does not acquire science data while inside the SAA polygon.

(A color version of this figure is available in the online journal.) 

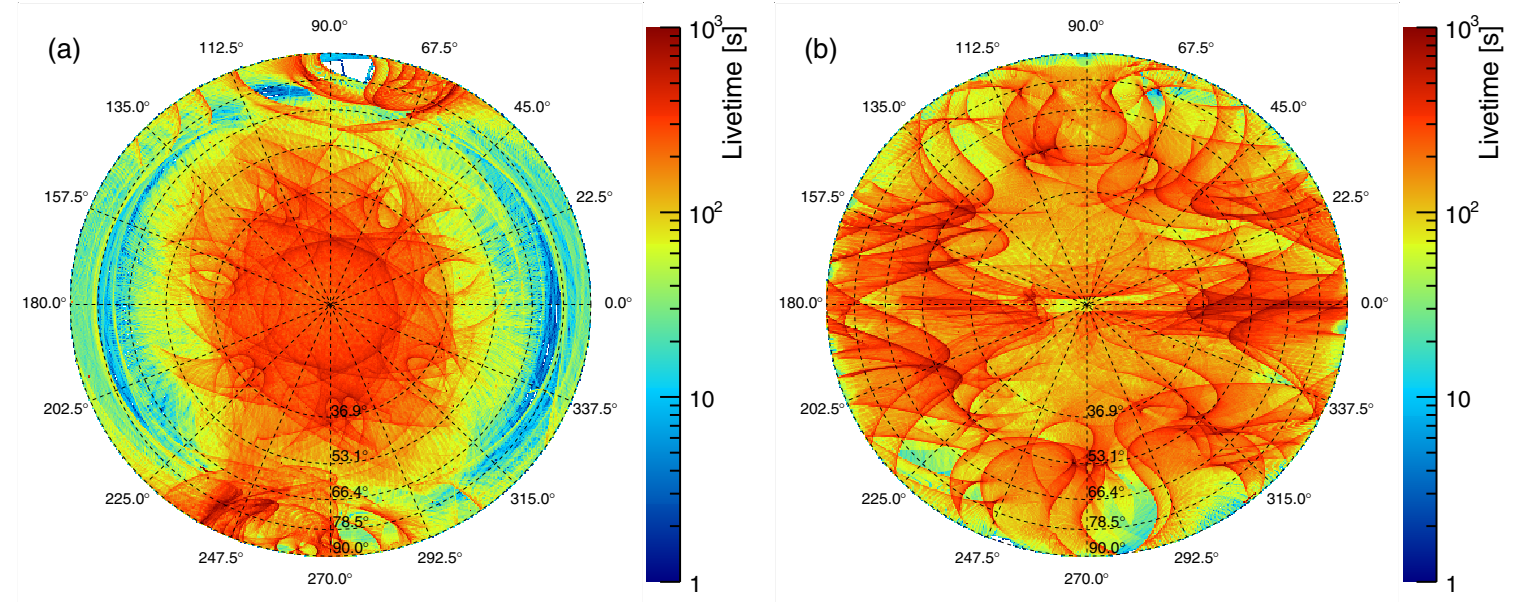

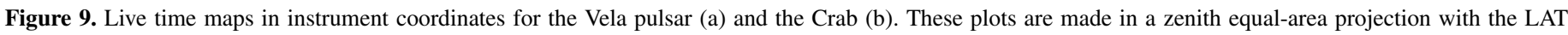

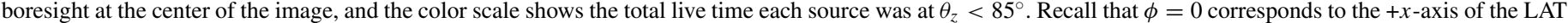
(Figure 1). These plots cover the same two-year time range that defines the standard calibration samples (see Section 3.6).

(A color version of this figure is available in the online journal.)

1. Digitization. We decompress the data and convert the information about signals in individual channels from the schema used in the electronics readout to more physically motivated schema-such as grouping signals in the ACD by tile, rather than by readout module.

2. Reconstruction. We apply pattern recognition and fitting algorithms commonly used in high-energy particle physics experiments to reconstruct the event in terms of individual TKR tracks and energy clusters in the CAL and to associate those objects with signals in the ACD (see Section 3.2).

3. Event analysis. We evaluate quantities that can be used as figures of merit for the event from the collections of tracks, clusters, and associated ACD information. Once we have extracted this information, we apply multivariate analysis techniques to extract measurements of the energy and direction of the event and to construct estimators that the event is in fact a $\gamma$-ray interaction (see Section 3.3).

4. Event selection. We apply the selection criteria for the various $\gamma$-ray event classes (Section 3.4).

In addition to these procedures, the processing pipeline is responsible for verifying the data integrity at each step and for producing and making available all the ancillary data products related to calibration and performance monitoring of the LAT.

On the whole, the LAT data processing pipeline utilizes approximately $130 \mathrm{CPU}$ years and $300 \mathrm{~TB}$ of storage each year.

The ISOC can reprocess data with updated algorithms from any stage in the process. However, given the quantities of data involved, reprocessing presents challenges both to the available pool of computing power and storage space. Table 2 gives a rough idea of the resources used by each stage in the process. Given the available resources, reprocessing from the Reconstruction step is practical only once a year or less often, and reprocessing from the Digitization step is feasible only every few years.

\subsection{Simulated Data}

In order to develop the filtering, reconstruction, and event selection algorithms described in Section 3, we used a detailed simulation of particle interactions with Fermi written in the Geant4 (Agostinelli et al. 2003) framework. This simulation includes particle interactions with a detailed material model
Table 2

Various Stages of the Data Processing Pipeline

\begin{tabular}{lcc}
\hline \hline Stage & $\begin{array}{c}\text { CPU Time } \\
\left(\mathrm{yr} \mathrm{yr}^{-1}\right)\end{array}$ & $\begin{array}{c}\text { Disk Usage } \\
\left(\mathrm{TB} \mathrm{yr}^{-1}\right)\end{array}$ \\
\hline Digitization & 1.2 & 26 \\
Reconstruction & 85 & 167 \\
Event analysis & 5 & 10 \\
Event selection & 1.3 & 60 \\
\hline Data monitoring & 25 & 12 \\
Calibration & 5 & 72 \\
\hline
\end{tabular}

Note. The numbers in the CPU time and disk usage columns refer to a typical year of data taking.

of Fermi, as well as simulations of the uncalibrated signals produced in the various sensors in the three subsystems.

The fact that the simulation is detailed enough to produce uncalibrated signals for each channel allows the simulations to be truly end-to-end.

1. We maintain fidelity to the analysis of the flight data by processing simulated data with the same reconstruction and analysis algorithms as the flight data.

2. On a related but slightly different note, we simulate the data as seen by the trigger and the on-board software, and process the data with a simulation of the hardware trigger and exactly the same on-board filter algorithms as used on the LAT.

3. We can merge the signals from two events into a single event. As described in Section 2.5.1, we rely on this feature to add an unbiased sample of ghost signals to simulated events.

\subsubsection{Ghosts and Overlaid Events}

The presence of ghost signals in the LAT data reduced the efficiency of the event selections tuned on simulated data lacking this effect, so we developed a strategy to account for the ghosts. One of the triggers implemented in the LAT data acquisition system, the PERIODIC trigger (see Section 3.1.1), provides us with a sample of ghost events: twice per second the LAT subsystems are read out independently of any other trigger 
condition. For the CAL and the ACD, all the channels, even those below threshold, are recorded (this is not possible for the TKR). Since these triggers occur asynchronously with the particle triggers, the ghost signals collected are a representative sample of the background present in the LAT.

We merge the signals channel-by-channel from a randomly chosen periodic trigger event into each simulated event. In more detail, we analyze the signals in each periodic trigger, converting the instrument signals to physical units; analogto-digital converter channels in the ACD and CAL, and time over threshold signals in the TKR, are converted into deposited energy. Since the intensity and spectrum of CRs seen by the LAT depend on the position of the Fermi satellite in its orbit, the ghost events are sorted by the value of McIlwain L (Walt 2005) at the point in the orbit where the event was recorded. Then, during the simulation process, for each simulated event we randomly select a periodic trigger event with similar McIlwain L, and add the energy deposits in this "overlay event" to those of the original simulated event, after which the combined event is digitized and reconstructed in the usual way.

We have used the improved simulations made with this overlay technique to more accurately evaluate the effective area of the LAT, and to better optimize the event selection criteria when developing Pass 7. Furthermore, we are studying ways to identify and tag the ghost signals and remove them from consideration in the event analysis (Rochester et al. 2010).

\subsubsection{Specific Source $\gamma$-Ray Signal Simulation}

We have developed interfaces between the software libraries that implement the flux generation and coordinate transformation used by the ScienceTools and our detailed Geant4-based detector simulation. This allows us to re-use the same source models that we use with the ScienceTools within our detailed Geant4-based detector simulation, insuring consistent treatment of Fermi pointing history and the source modeling. One application for the present work was simulating atmospheric $\gamma$ rays above $10 \mathrm{GeV}$ for comparison with our Earth limb calibration sample (see Section 3.6.3).

\subsubsection{Generic $\gamma$-Ray Signal Simulation}

In addition to simulating individual sources, we also simulate uniform $\gamma$-ray fields that we can use to explore the instrument response across the entire FoV and energy range of the LAT. These will henceforth be referred to as allGamma simulations, and they have the following features.

1. The $\gamma$ rays are generated with an $E^{-1}$ number spectrum (where $E$ is the $\gamma$-ray energy), i.e., the same number of $\gamma$ rays are generated in each logarithmic energy bin.

2 . The individual $\gamma$ rays are randomly generated on a sphere with $6 \mathrm{~m}^{2}$ cross-sectional area (i.e., large enough to contain the entire LAT and most of the spacecraft) centered at the origin of the LAT reference frame, i.e., the center of the TKR/CAL interface plane.

3. The directions of the $\gamma$-rays are sampled randomly across $2 \pi$ of downward-going (in the LAT reference frame) directions, so as to represent a semi-isotropic incident flux.

For defining the IRFs we simulate 200 million $\gamma$ rays over the range $\log _{10}(E / 1 \mathrm{MeV}) \in[1.25,5.75]$ and a further 200 million $\gamma$ rays over the range $\log _{10}(E / 1 \mathrm{MeV}) \in[1.25,2.75]$. The net result is to produce a data set that adequately samples the LAT energy band and FoV and that far exceeds the statistics of any single point source.

\subsubsection{Simulation of Particle Backgrounds}

In order to develop our event classification algorithms, and to quantify the amount of residual CR background that remains in each $\gamma$-ray event class, we require accurate models of both the CR background intensities and the interactions of those particles with the LAT. Accordingly, we use the same Geant4based detector simulation described in the previous section to simulate fluxes of the CR backgrounds.

There are three components to the CR-induced background in the LAT energy range.

1. Primary CRs. Protons, electrons, and heavier nuclei form the bulk of charged CRs. Due to the shielding by the magnetic field of the Earth, only particles with rigidities above a few GV (spacecraft position dependent) can reach the LAT orbit. Therefore, the background from primary CRs is relevant above a few GeV. The LAT event classification provides a very effective rejection of charged particles, up to an overall suppression factor of $10^{6}$ for CR protons (with a prominent contribution from the ACD). However, due to the intense flux of primary protons, a significant number of misclassified CRs enter even the P7SOURCE, P7CLEAN, and P7ULTRACLEAN $\gamma$-ray classes. Minimumionizing primary protons above a few $\mathrm{GeV}$ produce a background around $100-300 \mathrm{MeV}$ in the LAT. However, protons that inelastically scatter in the passive material surrounding the ACD (e.g., the micro-meteoroid shield) without entering the ACD can produce secondary $\gamma$ rays at lower energies, which are then indistinguishable from cosmic $\gamma$ rays, and which we refer to as irreducible background (see also Section 4.4).

2. Charged secondaries from $C R$ interactions. CRs entering the atmosphere produce a number of secondaries in interactions with the atmosphere itself. Charged particles produced in these interactions can spiral back out, constrained by the magnetic field of the Earth, and eventually re-enter the atmosphere. These particles are predominantly protons, electrons, and positrons and are an important backgrounds below the geomagnetic cutoff rigidity. Due to the high efficiency of the ACD in rejecting charged particles, the dominant contribution are to this background in the P7CLEAN and P7ULTRACLEAN event classes positron annihilations in the passive material around the ACD. The resulting $\gamma$ rays are again indistinguishable from cosmic $\gamma$ rays.

3. Neutral secondaries from CR interactions. $\gamma$ rays and neutrons are also produced in local CR interactions. Unaffected by the magnetic field, they approach the LAT from the direction of the Earth with intensities peaking at the Earth limb. Neutrons are easily suppressed by the event classification scheme and do not significantly contribute to the background. The $\gamma$-ray background is suppressed by pointing away from the Earth and excluding events that originate from near or below the Earth limb. Due to the long tails of the LAT PSF (see Section 6), however, a small fraction of the events originating from the Earth limb are reconstructed with directions outside the exclusion regions. Since the Earth limb is extremely bright (Abdo et al. 2009c), even this small fraction is an important residual background for celestial $\gamma$ rays. Since the PSF tails broaden with decreasing energy, the main contribution is at energies below a few hundred MeV (see Section 4.5). Similarly, a small fraction of $\gamma$ rays entering the LAT with incidence angles greater than $90^{\circ}$ are mischaracterized as downward-going 


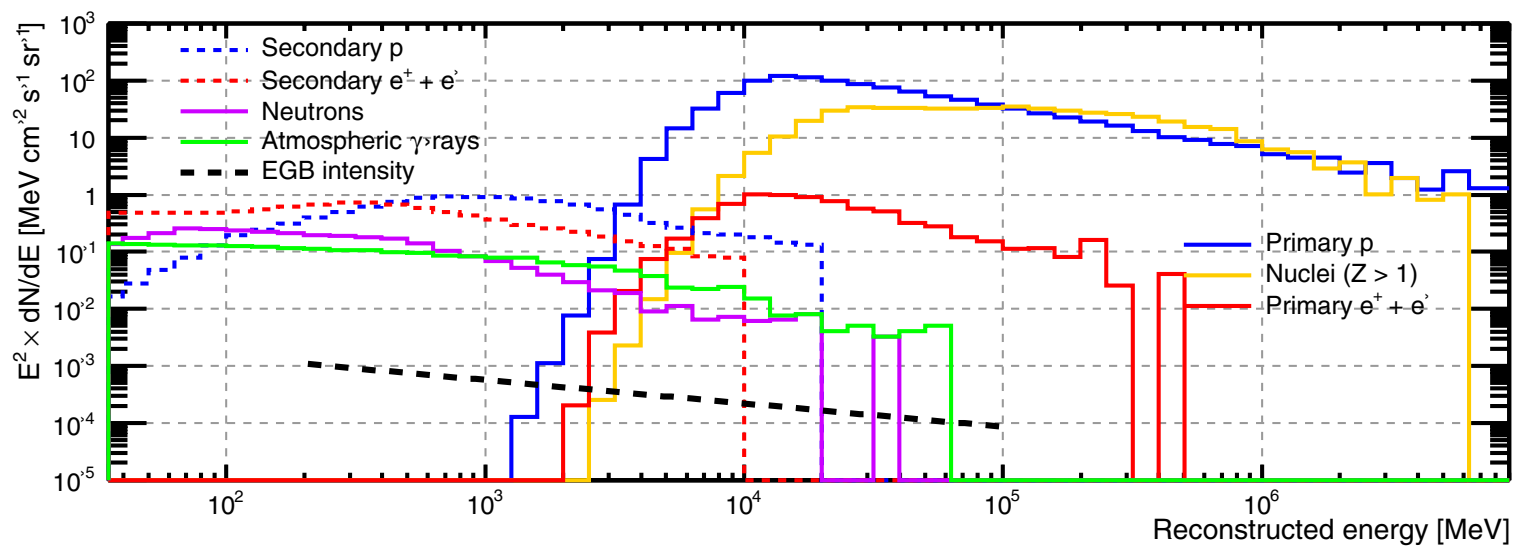

Figure 10. Model of the LAT orbital position and particle direction-averaged CR-induced particle intensities (Mizuno et al. 2004) sampled from a $64 \mathrm{~s}$ live-time background-simulation run. The intensity of the extragalactic diffuse background emission measured by the LAT (Abdo et al. 2010e) is shown for comparison. Note that the event energy is reconstructed under the hypothesis of a downward-going $\gamma$ ray and in general does not represent the actual energy for hadrons.

(A color version of this figure is available in the online journal.)

( $\sim 5 \%$ to $\sim 0.01 \%$, depending on the energy, incidence angle, and the event sample). Because the Earth limb is extremely bright and some part of it is almost always behind the LAT, $\gamma$ rays from the limb are the dominant component of "back-entering" background contamination. Furthermore, since we misestimate the directions of these back-entering $\gamma$ rays by $>90^{\circ}$, they are often reconstructed outside the Earth exclusion region.

Figure 10 shows the average $\mathrm{CR}$-induced particle intensities at the orbit of the LAT in the model that we use. For comparison the intensity of the extragalactic diffuse $\gamma$-ray emission measured by the LAT (Abdo et al. 2010e) is overlaid to demonstrate the many orders of background suppression necessary to distinguish it from particle background. The model was developed prior to launch based on data from satellites in similar orbits and balloon experiments (Mizuno et al. 2004).

As the particle rates are strongly dependent on location in geomagnetic coordinates, the details of the orbit model are also important. For tuning the event analysis, or for estimating the background rates for typical integration times of months or years, the simulated time interval must be at least equal to the precession period of the Fermi orbit (53.4 days). Simulating these high particle rates for such a long time interval is quite impractical, in terms of both CPU capacity and disk storage requirements. For studies of background rejection we usually simulate an entire precession period to ensure a proper sampling of the geomagnetic history, but to limit the particle counts we generate events for only a few seconds of simulated time every several minutes, e.g., a typical configuration requires event generation for $4 \mathrm{~s}$ every 4 minutes of time in orbit. This partial sampling is a compromise between the limited CPU and disk usage, and the requirement of having good statistics. Considering the LAT background rejection power, in order to have sizable statistics after even the first stages of the event analysis are performed, we must start with a simulated background data set of over $10^{9} \mathrm{CRs}$.

\section{EVENT TRIGGERING, FILTERING, ANALYSIS, AND CLASSIFICATION}

In this section, we describe the analysis steps that determine which events make it into our final $\gamma$-ray data sample, starting with the triggering and filtering performed by the on-board data acquisition system (Section 3.1), moving on to the reconstruction of particle interactions in the event (Section 3.2), the analysis of the event as a whole (Section 3.3), and finally the definition of the $\gamma$-ray classes (Section 3.4). The overall logical structure of this process is illustrated in Figure 11.

The event analysis requires knowledge of the LAT, the physics of particle interactions within its volumes, and of the particle backgrounds in the Fermi orbit. As described in Section 2.5, we use large MC samples of $\gamma$ rays and of CRs to devise the best procedures to extract estimates of energies and incident directions, and to classify events as either $\gamma$ rays or charged particle backgrounds.

Finally, in Section 3.5 we describe the publicly available LAT event samples, while in Section 3.6 we describe the calibration sources, event samples and methods we use to validate and characterize the performance of the LAT using flight data.

\subsection{Trigger and On-board Filter}

In this section, we review the event triggering, the readout of the LAT, and the filtering performed on board in order to reduce the data volume downlinked to ground.

\subsubsection{Triggering the LAT Readout}

Each subsystem provides one or more trigger primitives (or trigger requests) as detailed in the following list.

1. TKR (also known as "three-in-a-row"). Issued when three consecutive $x-y$ silicon layer pairs-corresponding to six consecutive silicon planes-have a signal above threshold (nominally $0.25 \mathrm{MIPs}$ ). This signals the potential presence of a track in a tower. Since many tracks cross between towers and/or have more than three $x-y$ layers within a tower, the TKR trigger request is very efficient.

2. CAL_LO. Issued when the signal in any of the CAL crystal ends crosses the low-energy trigger threshold (nominally $100 \mathrm{MeV}$ ).

3. CAL_HI. Issued when the signal in any of the CAL crystal ends crosses the high-energy trigger threshold (nominally $1 \mathrm{GeV})$.

4. VETO. Issued when the signal in any of the ACD tiles is above the veto threshold (nominally $0.45 \mathrm{MIP}$ ). It signals a charged particle crossing the tile. The trigger system has a programmable list of ACD tiles associated with each TKR 


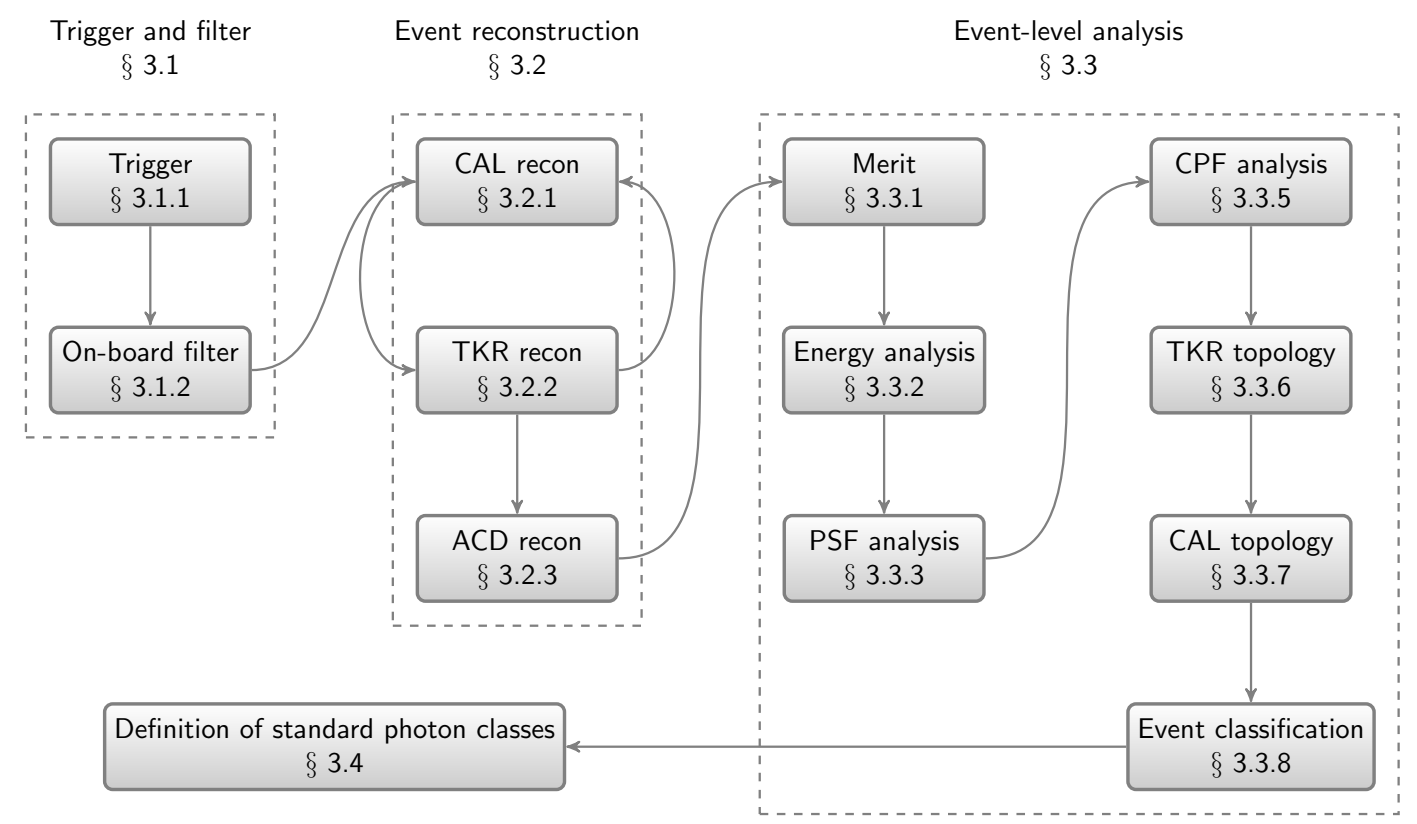

Figure 11. Logical structure of the analysis steps determining which events are selected for a given class. The references to section numbers are meant to help the reader navigate the content of Section 3.

tower; if ACD shadowing is enabled (which is the case for the nominal configuration) an additional ROI primitive is assembled when a TKR primitive in a tower happens in coincidence with a VETO primitive in the ACD tiles associated to the same tower.

5. CNO. Issued when the signal in any of the ACD tiles is above the CNO threshold (nominally 25 MIPs). It indicates the passage of a heavily ionizing nucleus (CNO stands for "carbon, nitrogen, oxygen") and it is primarily used for the calibration of the CAL (see Section 7.3.2).

The LAT has the ability to generate three other trigger primitives. We will refer to these as special primitives. Two of these are unused in flight and will not be discussed here. The third, the PERIODIC trigger, runs at a nominal frequency of $2 \mathrm{~Hz}$ during all science data taking and is used for diagnostic and calibration purposes.

We emphasize that although the trigger primitives provided by the TKR and the CAL are tower-based, a trigger initiates a readout of the whole LAT.

Trigger primitives are collected by the Central Trigger Unit. All 256 possible combinations of the eight trigger primitives are mapped into so-called trigger engines. In brief, some trigger requests are allowed to open a trigger window of fixed duration (nominally $700 \mathrm{~ns}$ ) and the set of primitives collected in this time interval is compared to a table of allowed trigger conditions. In the case where a trigger condition is satisfied, a global trigger (or trigger accept) is issued and event acquisition commences. The LAT readout mode (i.e., enabling the CAL and ACD zero suppression and selecting the CAL one-range or four-range readout) can be individually set for each trigger engine. In addition, trigger engines are scalable: for each trigger condition a prescale is specified, corresponding to the number of valid trigger requests necessary to issue a single global trigger (obviously no prescale is applied to engines intended for $\gamma$-ray collection).

The standard trigger engine definitions are listed in Table 3. Trigger engine 7 is particularly important for $\gamma$-ray events: it requires no special primitives, a three-in-a-row signal in the TKR (TKR), an absence of ROI shadowing and CNO from the
$\mathrm{ACD}$, and no CAL_HI. Engine 6 handles CAL_HI events and engine 9 handles events with enough energy deposition in the CAL to potentially cause ROI shadowing.

Trigger engine 4 is typical for calibration data (e.g., heavy ion collection): it requires no special primitives, three-in-a-row in the TKR (TKR), a highly ionizing passage in the ACD (CNO) in close correspondence (ROI), and at least a moderate energy deposition in the CAL (CAL_LO). Engines 5 and 10 process very few $\gamma$ rays and are used primarily for calibration and monitoring, so they are prescaled to limit the readout rate and/or to match the downlink bandwidth allocation.

\subsubsection{Event Filtering}

As mentioned before, since limited telemetry bandwidth is available for data downlink, some event filtering is required. The on-board filter allows the coexistence of different filtering algorithms and, in fact, in the nominal science data taking configuration, all the events are presented to three different filters.

1. The GAmma filter, designed to accept $\gamma$ rays (with an output average rate of approximately $350 \mathrm{~Hz}$ ).

2. The HIP filter, designed to select heavy ion event candidates, primarily for CAL calibration (with an output average rate of approximately $10 \mathrm{~Hz}$ ).

3. The Diagnostic filter, designed to enrich the downlinked data sample in events useful to monitor sensor performance and selection biases: specifically $\sim 2 \mathrm{~Hz}$ of periodic triggers and an unbiased sample of all trigger types prescaled by a factor 250 (with an output average rate of approximately $20 \mathrm{~Hz}$ ).

The GAMmA filter consists of a hierarchical sequence of veto tests, with the least CPU-intensive tests performed first. If an event fails a test, it is marked for rejection and not passed on for further processing (however, a small subset of events that are marked for rejection are downlinked through the DIAGNOSTIC filter). Some of the tests utilize rudimentary, two-dimensional tracks found by the on-board processing. The GAMMA filter has several steps (listed in order of processing). 
Table 3

Definition of the Standard Trigger Engines

\begin{tabular}{|c|c|c|c|c|c|c|c|c|}
\hline Engine & PERIODIC & CAL_HI & CAL_LO & TKR & ROI & CNO & Prescale & $\begin{array}{c}\text { Average Rate } \\
(\mathrm{Hz})\end{array}$ \\
\hline 3 & 1 & $\times$ & $\times$ & $x$ & $\times$ & $\times$ & 0 & 2 \\
\hline 4 & 0 & $x$ & 1 & 1 & 1 & 1 & 0 & 200 \\
\hline 5 & 0 & $x$ & $x$ & $x$ & $x$ & 1 & 250 & 5 \\
\hline 6 & 0 & 1 & $x$ & $x$ & $x$ & 0 & 0 & 100 \\
\hline 7 & 0 & 0 & $\times$ & 1 & 0 & 0 & 0 & 1500 \\
\hline 8 & 0 & 0 & 1 & 0 & 0 & 0 & 0 & $400^{\mathrm{a}}$ \\
\hline 9 & 0 & 0 & 1 & 1 & 1 & 0 & 0 & 700 \\
\hline 10 & 0 & 0 & 0 & 1 & 1 & 0 & 50 & 100 \\
\hline
\end{tabular}

Notes. The table lists the primitives used (1: required; 0: excluded; $\times$ : either), prescale factors, and typical rates for each of the trigger engines. In this short-hand representation, engines are defined with the highest precedence first: each combination of trigger primitives is mapped to the engine corresponding to the first condition it matches. Trigger engines 0,1 , and 2 are used by the LAT software for bookkeeping and to catch conditions that should not happen on orbit.

${ }^{a}$ In the nominal configuration for science data taking the CAL_LO condition is inhibited from opening a trigger window, and therefore engine 8 is effectively disabled.

1. Reject events that have patterns of ACD tile hits that are consistent with CRs and do not have the CAL_LO trigger primitive asserted, making it unlikely that the ACD hits were caused by backsplash.

2. Accept all events for which the total energy deposited in the CAL is greater than a programmable threshold, currently set to $20 \mathrm{GeV}$.

3. Reject events that have ACD hit tile patterns that are spatially associated with the TKR towers that caused the trigger, provided that the energy deposited in the CAL is less than a programmable threshold (currently set to $350 \mathrm{MeV}$ ).

4. Reject events for which a significant energy deposition in the CAL (typically $>100 \mathrm{MeV}$ ) is present but the pattern of hits in the TKR is unlikely to produce any track.

5. Reject events for which rudimentary tracks match with individual ACD tiles that were hit, provided the energy deposited in the CAL is less than some programmable amount (typically $5 \mathrm{GeV}$ ).

6. Reject events that do not have at least one rudimentary track.

Although it may seem strange to apply the requirement that there be any tracks after cutting due to matches between tracks and the ACD, recall that the on-board filter software is highly optimized for speed, and terminates processing of each event as soon as it is possible to reach a decision. Thus, the testing for track matches is performed during the track-finding stage, at the time the tracks are constructed.

\subsection{Reconstruction Algorithms}

Event reconstruction translates the raw event information from the LAT subsystems into a high-level event description under the assumption of a $\gamma$ ray impinging on the LAT volumes within $90^{\circ}$ of the boresight (see Figure 12 for an illustrative event display).

Here we will briefly summarize the event reconstruction algorithms underlying both Pass 6 and Pass 7 flavors of the event analysis; a more detailed description is given in Atwood et al. (2009). We want to stress that Pass 6 and Pass 7 use exactly the same reconstruction algorithms with the exception of the energy unbiasing (described in Section 3.2.1), which was only applied to Pass 7 data.

\subsubsection{Calorimeter Reconstruction}

The starting point for the energy evaluation is the measured energy depositions in the crystals. The centroid of the energy deposition is determined and the principal axes of the shower are evaluated by means of a principal moment analysis. In the Pass 6 and Pass 7 event reconstruction procedure, the energy deposition is treated as a single quantity, with no attempt to identify contamination from ghost signals. Work to develop an algorithm to separate the CAL energy deposition into multiple clusters and to disentangle ghost signals is ongoing (Rochester et al. 2010). The amount of energy deposited in the TKR is evaluated by treating the tungsten-silicon detector as a sampling calorimeter; this contribution is an important correction at low energies.

We apply three algorithms to estimate the actual energy of an event: a parametric correction (PC), a fit of the shower profile (SP), and a maximum likelihood (LH) approach. The energy assigned to any given event is the energy from one or the other of these algorithms. The three methods were designed to provide the best performance in different parts of the energy and incidence angle phase space (in fact, the LH algorithm was only tuned up to $300 \mathrm{GeV}$, while the SP algorithm does not work well for events below $\sim 1 \mathrm{GeV}$ ). Accordingly, they provide different energy resolutions and their distributions have slightly different biases (i.e., the most probable values are slightly above or below the true energy) for different energies and incidence angles; more details can be found in Atwood et al. (2009).

In fact, the only significant change in the Pass 7 event reconstruction relative to Pass 6 is to apply separate corrections for the biases of each energy estimation algorithms. We used MC simulations to characterize the deviations of the most probable value of the energy dispersion from the true energy across the entire LAT phase space for the three methods separately. Such deviations were found to be typically of the order of a few percent (with a maximum value of $\sim 10 \%$ ) and always significantly smaller than the energy resolution-with LH displaying a negative bias and PC and SP displaying a positive bias in most of the phase space.

We generated correction maps (as functions of $\gamma$-ray energy and zenith angle) and in Pass 7 the residual average bias for all the inputs of the final energy assignment (discussed in Section 3.3.2) is less than $1 \%$ in the entire LAT phase space. 

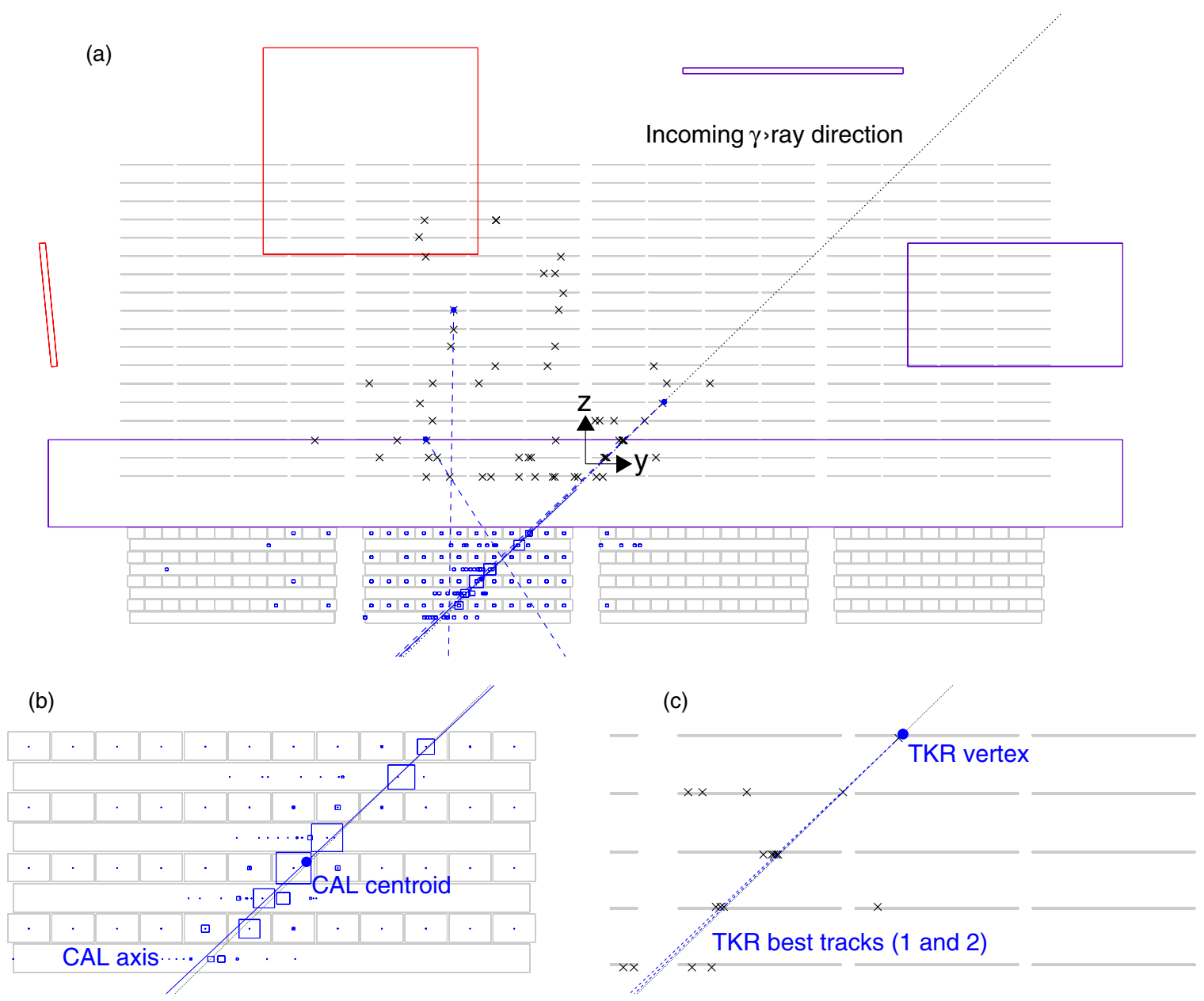

Figure 12. Event display of a simulated $27 \mathrm{GeV} \gamma$ ray (a) and zoom over the CAL (b) and TKR (c) portions of the event. The small crosses represent the clusters in the TKR, while the variable-size squares indicate the reconstructed location and magnitude of the energy deposition for every hit crystal in the CAL. The dotted line represents the true $\gamma$-ray direction, the solid line is the CAL axis (Section 3.2.1), and the dashed lines are the reconstructed TKR tracks (Section 3.2.1). The backsplash from the CAL generates tens of hits in the TKR, with two spurious tracks reconstructed in addition to the two associated with the $\gamma$ ray (note that they extrapolate away from the CAL centroid and do not match the CAL direction). It also generates a few hits in the ACD, which, however, are away from the vertex direction extrapolation and therefore do not compromise our ability to correctly classify the event as a $\gamma$ ray.

(A color version of this figure is available in the online journal.)

\subsubsection{Tracker Reconstruction}

For the TKR we merge and assemble clusters of adjacent hit strips into track candidates by combinatorial analysis. We have developed two methods: for CAL-seeded pattern recognition (CSPR) the trajectory of the original $\gamma$ ray is assumed to point at the centroid of the energy released in the CAL; the blind search pattern recognition (BSPR) can be used when there is little or no energy deposit in the CAL sensitive volumes.

Both the CSPR and BSPR algorithms start by considering nearby pairs of TKR clusters in adjacent layers as candidate tracks (the CSPR algorithm limits the candidate tracks to those pointing toward the CAL energy centroid). Both algorithms then proceed by using a Kalman filtering technique (see Kalman 1960; Fruhwirth 1987, for details about Kalman filtering and its use in particle tracking) which tests the hypotheses that additional TKR clusters were generated by the same incident particle and should be associated with each of the candidate tracks, and adds any such clusters to the appropriate candidate tracks. Furthermore, as each cluster is added to candidate tracks the Kalman filter updates the estimated direction and associated covariance matrix of those tracks. Both the CSPR and BSPR algorithms are weighted to consider the best candidate track to be the one that is both pointing toward the CAL centroid and is the longest and straightest. In the CSPR, the main axis of the CAL energy deposition is also considered, candidate tracks for which the TKR and CAL estimated directions differ significantly are disfavored starting at $\sim 1 \mathrm{GeV}$, and increasingly so at higher energies. At the completion of the CSPR algorithm the best candidate track is selected and confirmed as a track, and the clusters in it are flagged as used. We iterate the CSPR algorithm until no further tracks can be assembled from the unused TKR clusters, then proceed with the BSPR.

If more than one track is found in a given event, we apply a vertexing algorithm that attempts to compute the most likely common origination point of the two highest quality (i.e., longest and straightest) tracks, and, more importantly, to use that point as a constraint in combining the momenta of the two tracks to obtain a better estimate of the direction of the incoming $\gamma$ ray.

\subsubsection{ACD Reconstruction}

The ACD phase of the event reconstruction starts by estimating the energy deposited in each of the tiles and ribbons. Subsequently, these energy depositions are associated with each 
of the tracks found in the TKR. More specifically, each track is projected onto the ACD, and we calculate whether that track intersects each ACD tile or ribbon with non-zero energy deposition. Furthermore, if the track projection does not actually cross an ACD element with non-zero energy deposition, we calculate the distance of closest approach between the track projection and the nearest such ACD element. Finally, we use the distance calculation and the amount of energy deposited to sort all the possible ACD-TKR associations by how likely they are to represent a charged particle passing through the ACD and into the TKR. This ranking is used when considering whether the event should be rejected in later analysis stages.

\subsection{Event Analysis}

The first step of the event-level analysis procedure is to extract simpler representations of the event characteristics from the complex structures assembled during the reconstruction phase. These high-level quantities are then used for the final assignment of the event energy and direction (among the outputs of the reconstruction algorithms described in Section 3.2) and for the background rejection. The final product is an event-by-event array of simple quantities relevant for scientific analysis: energy, direction, and estimates of the probability a given event is a $\gamma$ ray $\left(P_{\text {all }}\right)$.

\subsubsection{Extraction of "Figure-of-merit" Quantities}

For each event, we reduce the output of the TKR, CAL, and ACD reconstruction to a set of a few hundred figure-ofmerit quantities whose analyzing power has been studied and optimized with MC simulations.

It is important to note that the best track (and to a lesser extent the second best track) plays a particularly important role in later analyses. Specifically, although we calculate figure-of-merit variables - such as the agreement between the track direction and the axis of the CAL shower, or the distance between the track extrapolation and the nearest ACD energy deposition-for all the tracks in the event, many of the multivariate analyses described in the rest of this section consider only those figureof-merit variables associated with the two best tracks in the event. Furthermore, the figure-of-merit variables associated with the best track tend to carry significantly more weight in the multivariate analysis - which is of primary importance, as most ghost tracks come from protons and heavy ions and tend to be longer and straighter than tracks from $e^{+}$and $e^{-}$from a $\gamma$-ray conversion.

Early iterations of the event analysis split the events by energy and then applied different selections in the various energy bands. We found that this approach led to large spectral features at the bin boundaries. Therefore, we chose instead to scale many of our figure-of-merit variables with energy so as to remove most of the energy dependence. This allowed us to have a single set of event selection criteria spanning the entire LAT energy range.

\subsubsection{Event Energy Analysis}

The second step in the event analysis is selecting one energy estimate among those available for the event (see Section 3.2.1). We apply a classification tree (CT) analysis (Breiman et al. 1984; Breiman 1996) to select which of the energy reconstruction methods is most likely to provide the best estimate of the event energy.

Because of the numerous edges and gaps between the logs in the CAL, and because of the huge energy range and large FoV of the LAT, the quality of the energy reconstruction can vary significantly from event to event. Therefore, for each event we also apply a second CT analysis to estimate the probability that the estimated energy is within the nominal core of the energy dispersion. Specifically, we define a scaled energy deviation, as described in more detail in Section 7.1.1, from which most of the energy and angular dependence is factored (i.e., the energy dispersion in the scaled deviation is largely energy and angle independent). We then train CTs that provide probability estimates this event is less than $2 \sigma\left(P_{2 \sigma}\right)$ or $3 \sigma\left(P_{3 \sigma}\right)$ away from the most probable value of the energy dispersion. Finally, we define a reconstruction quality estimator $P_{\mathrm{E}}$ by combining $P_{2 \sigma}$ and $P_{3 \sigma}$ :

$$
P_{\mathrm{E}}=\sqrt{P_{2 \sigma} P_{3 \sigma}} .
$$

Large values of $P_{\mathrm{E}}$ indicate that the event is likely to be in the core of the energy dispersion, and so have an accurate energy estimate.

In the Pass 7 analysis we did not use the energy estimates from the LH algorithm: by construction it is a binned estimator, and energy assignments tended to pile up at the bin boundaries (see Section 7.2 for a more detailed discussion of the effects of removing the $\mathrm{LH}$ algorithm from consideration in the energy assignment). The removal of the LH energies causes a somewhat degraded energy resolution for those events where it would have been selected; we compensate for the loss of this energy estimator by requiring a slightly more stringent cut on the energy reconstruction quality when defining the standard event classes (e.g., see Section 3.4.3).

\subsubsection{Analysis of the Event Direction}

The third step selects the measured direction of the incoming $\gamma$ ray from the available options. Those options are the directions as estimated from (1) the best track, (2) the best vertex, and (3) and (4) the same two options using the centroid of the energy deposition in the CAL as an additional constraint on the direction of the incident $\gamma$ ray. Again, we use a CT analysis to combine the information about the event and determine which of the methods is most likely to provide the best direction measurement.

As with the energy analysis, the quality of the direction reconstruction can vary significantly from one event to the next. In this case, we have the additional complication that $\gamma$ rays can convert at different heights in the TKR, giving us anywhere between 6 and 36 track position measurements. Therefore, we use an additional CT analysis to estimate the probability $P_{\text {core }}$ that the reconstructed direction falls within the nominal $68 \%$ containment angle as defined by the simplified analytical model:

$$
C_{68}(E)=\sqrt{\left[c_{0} \cdot\left(\frac{E}{100 \mathrm{MeV}}\right)^{-\beta}\right]^{2}+c_{1}^{2}}
$$

(where the values of the coefficients for front- and backconverting events are listed in Table 4). The reader will notice the similarity with the functional expression used for the PSF prescaling described in Section 6.1.

\subsubsection{Differences in Event Energy and Direction Analyses between Pass 6 and Pass 7}

There is a particular subtlety to the event reconstruction that merits a brief discussion. Specifically, the different event reconstruction algorithms we use provide a set of choices for the best energy and direction of each event. The stages of the eventlevel analysis described in the previous two subsections select 

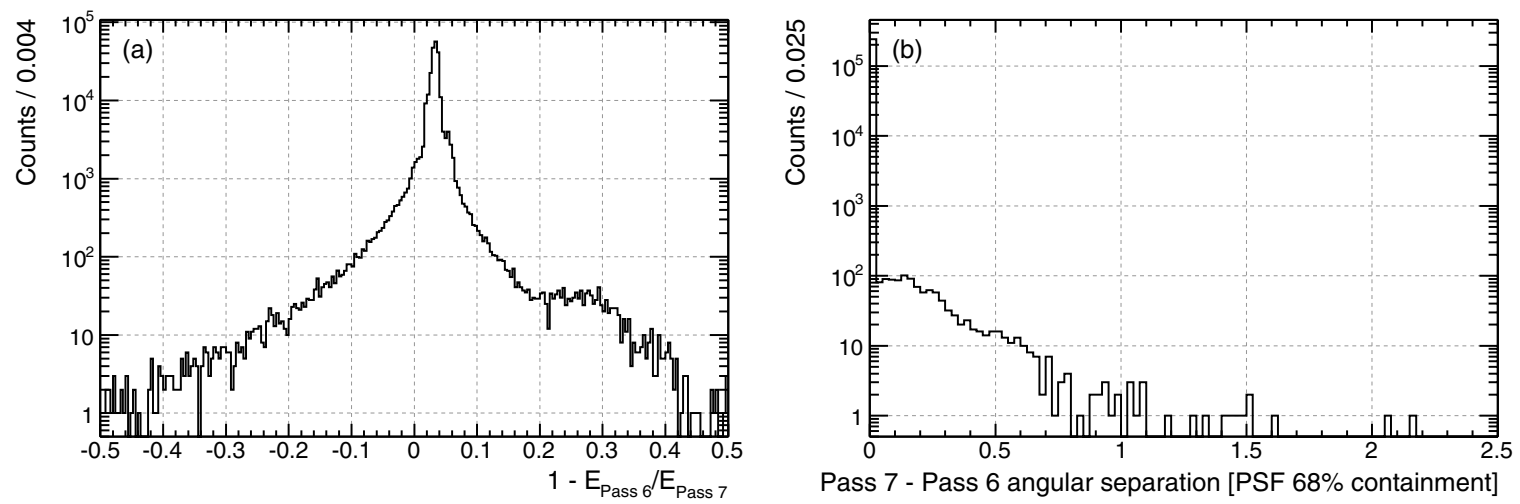

Figure 13. Event-by-event differences in the reconstructed energy (a) and direction (b) between Pass 6 and Pass 7 for a sample of P7SOURCE events above 100 MeV. The two histograms are based on a week of data, with a zenith angle cut at $100^{\circ}$ to remove the bright Earth limb emission, typically viewed at a large angle in LAT coordinates. In order to factor out the energy dependence of the PSF, the angular separation is measured in units of nominal 68\% containment at the energy of the event. Note that in the vast majority of the cases the same direction is assigned in Pass 6 and Pass 7. On the other hand, the systematic offset in energy is due to the energy unbiasing introduced in Pass 7 (Section 3.2.1).

Table 4

Coefficients of the Analytical Model for the 68\% Containment Angle for the PSF from Equation (5) Used for the Multivariate Analysis Used to Evaluate the Quality $p_{\mathrm{CORE}}$ of the Direction Reconstruction

\begin{tabular}{llll}
\hline \hline Conversion Type & $c_{0}$ & $c_{1}$ & $\beta$ \\
& $\left(^{\circ}\right.$ & $\left(^{\circ}\right.$ & \\
\hline Front & 3.3 & 0.1 & 0.78 \\
Back & 6.6 & 0.2 & 0.78 \\
\hline
\end{tabular}

the algorithms that are more likely to provide the best estimates. Therefore, although the event reconstruction was unchanged except for the energy unbiasing, changes in the event-level analysis can result in individual events being assigned slightly different directions and/or energy estimates in Pass 7 with respect to Pass 6. Figure 13 illustrates such differences for the P7SOURCE events above $100 \mathrm{MeV}$ that were also included in the P6_DIFFUSE $\gamma$-ray class.

\subsubsection{Rejection of Charged Particles in the Field of View}

The next step of the event analysis starts the process of classifying events as $\gamma$ rays or particle backgrounds, specifically identifying events for which evidence clearly indicates that a charged particle entered the LAT from inside the FoV. We refer to this stage as the charged particle in the FoV (CPF) analysis. To accomplish this, we first apply a series of filters that classify as background those events for which the best track has hits all the way to the edge of the TKR and (1) points to an active region of the ACD that has significant deposited energy or (2) points directly to less sensitive areas in the ACD, such as the corners or gaps between ACD tiles.

After applying these filters we attempt to account for cases where the best reconstructed track does not represent the incoming particle well. This can happen for a variety of reasons, for example, when the bias of the track-finding algorithm toward the longest, straightest track causes us to incorrectly assign a backsplash particle from the CAL as the best track, or when the incoming particle passes largely in the gaps between the TKR towers and we incorrectly select some secondary particle as the best track. We classify as background the events for which the total energy deposited in the ACD is too large to be accounted for as backsplash from the CAL. It is important to note that this particular requirement loses the benefit of the ACD segmentation, so we apply a conservative version to all events, and a tighter version to events that have a track that passes within an energy-dependent distance of significant energy deposition in the ACD. During extremely bright SFs this total ACD energy requirement can cause us to misclassify many $\gamma$ rays as CRs because of energy deposition in the ACD from SF X-rays (see Appendix A).

All the events that are classified as charged particles at this stage are removed from further consideration as $\gamma$-ray candidates and are passed to separate analyses to identify various species of charged particles (see, e.g., Abdo et al. 2009b; Ackermann et al. 2010, 2012a, 2012b).

Finally, we perform another CT analysis to combine all the available information into an estimate of the probability that the event is from charged particle backgrounds $\left(P_{\mathrm{CPF}}\right)$. Although the performance of this selection depends on energy and angle, roughly speaking more than $95 \%$ of the background in the telemetered data is removed by means of these cuts, and about $10 \%$ of the $\gamma$-ray sample is lost.

We note in passing that electrons and positrons cause electromagnetic showers that look extremely similar to $\gamma$-ray interactions, and the remaining stages of the event analysis are based on the topology (e.g., the shape, density, and smoothness) of the energy deposition in the TKR (Section 3.3.6) and CAL (Section 3.3.7), and have very little additional discriminating power against such backgrounds.

\subsubsection{TKR Topology Analysis}

In the next stage of the event analysis, we use information from the TKR to identify CR backgrounds that were not identified as such by the CPF analysis. These events are not immediately removed from the $\gamma$-ray analysis but only flagged to allow for removal from the higher purity event classes. In this part of the analysis, we flag events saturating the energy deposition in the TKR planes with a pattern expected for heavily ionizing particles as well as events with very high energy deposition in the first hit layers of the TKR, which is a signature of a particle coming from the CAL that ranges out in the middle of the TKR.

After these two flags are applied, we divide the events among five branches for evaluating their topologies in the TKR: the first one collects events with a vertex solution in the TKR, the remaining ones separate events with one track and events with many tracks, treating separately events converting in the thin and thick section of the TKR. This is done because each of these 
different topologies has significantly different ratios of $\gamma$-ray signal-to-background contamination, and presents accordingly different difficulty for selecting $\gamma$ rays. For each branch we apply a different initial selection to remove many CR background events and then pass the remaining events to a CT analysis, which estimates the probability $\left(P_{\mathrm{TKR}}\right)$ the event comes from $\gamma$-ray interactions as opposed to charged particle backgrounds (and more specifically hadronic charged particle backgrounds).

The variables used in the CT analysis were designed to emphasize differences between the characteristics of $\gamma$-ray conversions and hadronic CRs. This includes distinguishing between electromagnetic and hadronic showers by counting extra hits near the track, quantifying the complexity of the event in the TKR and how collimated the overall track structure is, looking how deep into the fiducial volume the event starts, and requiring that the ionization along the track is consistent with $e^{ \pm}$pairs.

\subsubsection{CAL Topology Analysis}

Next we apply a CAL topology analysis that is similar in design to the TKR topology analysis. The first part of this analysis consists of few general cuts flagging events coming from the bottom and sides of the LAT. As with the TKR topology analysis, we then split the events into five branches depending on the topology; for each branch, we apply a cut and a CT stage. Again, CTs are trained to select $\gamma$ rays versus hadronic charged particle backgrounds, and provide an estimate of the probability that the event is a $\gamma$ ray $\left(P_{\mathrm{CAL}}\right)$.

The variables used in the CAL topology analysis are not only CAL-derived quantities, but also involve comparisons between what is observed in the CAL and what is seen in the TKR. Among the important discriminants are how well the track solution points toward the CAL energy centroid, how well the direction derived from the CAL (via the moments analysis, see Section 3.2.1) agrees with the track direction, the root mean square (rms) width of the CAL shower, and the ratio of the highest energy in a single crystal to the total energy in all crystals.

\subsubsection{Event Classification}

After the analyses related to the three LAT subsystems, the last stage of our event analysis applies a final CT analysis utilizing all available information, notably including the output of the CT analyses from previous phases of the event analysis. This second-order CT analysis is particularly important for defining event classes with high $\gamma$-ray purity, as discussed in Section 3.4.

At this point, we have a number of specifiers of event reconstruction and classification quality on an event-by-event basis.

1. Energy reconstruction quality $P_{\mathrm{E}}$ (see Section 3.3 .2 ).

2. Direction reconstruction quality $P_{\text {core }}$ (see Section 3.3.3).

3. $\gamma$-ray probabilities from the $\mathrm{CPF}$ analysis $\left(P_{\mathrm{CPF}}\right.$, see Section 3.3.5) and the TKR $\left(P_{\mathrm{TKR}}\right.$, see Section 3.3.6) and CAL $\left(P_{\mathrm{CAL}}\right.$, see Section 3.3.7) topology analysis.

4. Overall $\gamma$-ray probability $P_{\text {all }}$ from the final classification step of the event analysis.

These are the basic ingredients for defining the standard $\gamma$-ray classes described in Section 3.4.

\subsection{Standard Event Classes for $\gamma$ Rays}

Making use of the classification quantities described in the preceding subsections, we define event classes optimized for a

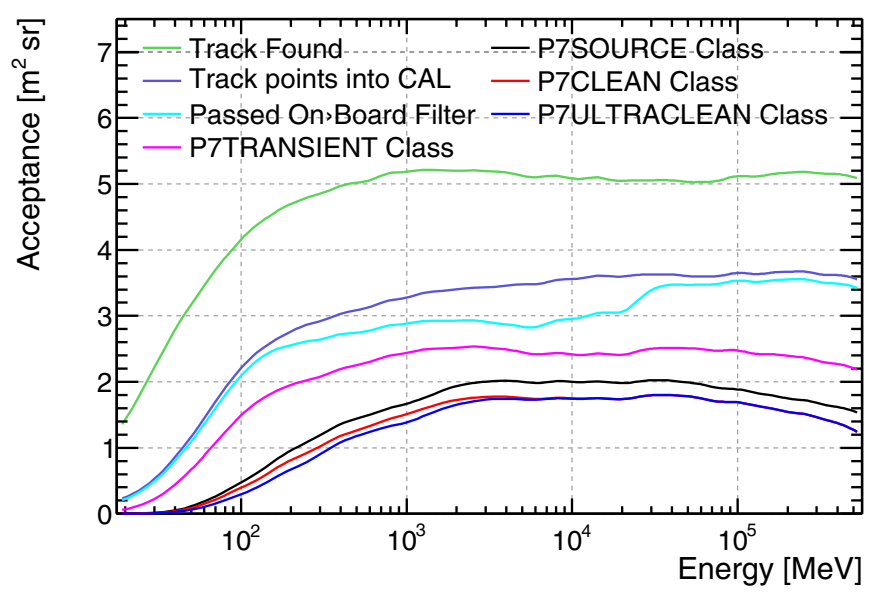

Figure 14. LAT $A_{\text {eff }}$ integrated over the FoV as a function of energy at successive stages of the event filtering as estimated with simulated data. Since we require a direction and energy to use a $\gamma$ ray for science analysis, we consider only events with at least one track found and that pass the fiducial cuts (see Section 3.4.1). (A color version of this figure is available in the online journal.)

range of astrophysical source analyses. We note that these event classes are nested: each succeeding selection is a strict subset of the previous one. The relative selection efficiencies for the event classes as well as previous stages of the event selection process are shown in Figure 14.

\subsubsection{Track-finding and Fiducial Cuts}

For the definition of the standard $\gamma$-ray classes we confine ourselves to events that have some chance to be useful for most standard science analysis, namely events for which we have enough information to reconstruct an energy and a direction. Therefore, we require that the event has a reconstructed track to allow for a direction estimation. Furthermore, we require that the track points into the CAL and crosses at least 4 radiation lengths of material in the CAL and that at least $5 \mathrm{MeV}$ of energy is deposited in the CAL. This second requirement reduces the fiducial volume of the detector by rejecting off-axis $\gamma$ rays that pass near the top of the TKR and miss the CAL. Events with no tracks in the TKR or with less energy deposited in the CAL are not considered further as candidate $\gamma$ rays. The remaining data set (i.e., the events that are passed along as potential candidates for the standard $\gamma$-ray classes) is still composed almost entirely of background events.

We note that these cuts remove from consideration two classes of events that might be useful for specific, non-standard, analyses. The first class consists of events that deposit all their energy in the TKR, either because they range out before they reach the CAL or simply because they miss the CAL entirely. In general, the energy resolution is much poorer for these events, though at low energies $(<100 \mathrm{MeV})$ the CAL does not improve the energy resolution significantly. These events have been used effectively in the analysis of both gamma-ray bursts (GRBs; Pelassa et al. 2010) and SFs (Ackermann et al. 2012c). The second class consists of events that do not have reconstructed tracks, but have enough information in the CAL to derive an estimate of the event direction (though without the TKR information the angular resolution for these events is highly degraded). These events can occur because the $\gamma$ ray entered the LAT at a large incidence angle and missed most of the TKR, or simply because the $\gamma$ ray passed through the TKR without converting. 


\subsubsection{P7TRANSIENT Class Selection}

For the analysis of brief transient sources (e.g., GRBs) a high purity is not required as the time selection itself limits the amount of background counts in the region of interest (ROI). Accordingly, we define a P7TRANSIENT event class with only a few cuts, with the aim of achieving a residual background rate of a few $\mathrm{Hz}$ while maintaining a large efficiency for $\gamma$ rays. The list of cuts is short.

1. The event must pass minimal cuts on the quality estimators $P_{\mathrm{E}}$ and $P_{\text {core }}\left(P_{\mathrm{E}}>0\right.$ and $\left.P_{\text {core }}>0\right)$.

2. The event energy after all corrections must be greater than $10 \mathrm{MeV}$.

3. The event energy after all corrections must not amount to more than five times the energy deposited in the CAL.

4. The CPF analysis must not flag the event as a charged particle $\left(P_{\mathrm{CPF}}>0.1\right)$.

5. The event must pass a relatively loose cut on $P_{\text {all }}\left(P_{\text {all }}>\right.$ $0.2)$.

It is worth noting that although the cuts are optimized somewhat differently in Pass 6, a similar series of cuts are applied to define the P6_TRANSIENT event class, which is roughly equivalent to the P7TRANSIENT event class.

\subsubsection{P7SOURCE Class Selection}

We have several additional considerations when defining the P7SOURCE event class, which is intended for the analysis of point sources. While the $\gamma$ rays from a point source are clustered on the sky near the true source position, residual CR background can be modeled as an isotropic component and be accounted for in the analysis of the spectrum or position of the source. Although the exact numbers depend on the details of the PSF and the spectrum of the source, in general we require a background rate of less than $\sim 1 \mathrm{~Hz}$ in the LAT FoV to ensure that we maintain a high enough signal-to-background ratio so that this has little impact on source detection and characterization. Furthermore, in contrast to studies of transient sources, for sources with an integral energy flux above $100 \mathrm{MeV}$ of $\sim 10^{-11} \mathrm{erg} \mathrm{cm}^{-2} \mathrm{~s}^{-1}$, the precision of spectral studies is often limited by systematic uncertainties below $1 \mathrm{GeV}$ (see Section 8.3), increasing the importance of the precision and accuracy of the event energy and direction reconstruction.

Additionally, we note that for the P7SOURCE event class and both higher purity event classes (P7CLEAN and P7ULTRACLEAN), we developed and optimized the cuts using on-orbit data samples (particularly the clean and dirty samples described in Section 3.6.4) as well as MC simulations. In particular, we performed a comparison of many event quality parameters and reconstruction variables between the clean and dirty samples to identify characteristics of the CR background, which is greatly enhanced in the dirty sample, and devised selection criteria to remove it. Some examples of this procedure are described in more detail in Section 4.3.

An important limitation of this technique is obviously that one needs a very low ratio of residual CR background to $\gamma$ rays in the clean sample to obtain large contrast between the clean and dirty samples. It can only be applied to data samples which already have a $\gamma$-ray-to-residual-background ratio that is comparable to 1 . Therefore, when optimizing these selections we only used events that are part of the P7TRANSIENT class (see Section 3.4.1) and additionally have a high value of $P_{\text {all }}$ estimator introduced in Section 3.3.8.
The P7SOURCE event class is defined starting from the P7TRANSIENT event class and includes tighter cuts on many of the same quantities. Specifically, the P7SOURCE event class selection requires the following.

1. The event must not have been flagged as background based on topological analysis of the CAL (Section 3.3.6) and TKR (Section 3.3.6) reconstruction, and must pass a tighter cut on $P_{\mathrm{E}}$; the cut on $P_{\mathrm{E}}$ does reject a noticeable number of events, though by definition these events have less accurate energy estimates on average $\left(P_{\mathrm{E}}>0.1\right.$ or $P_{\mathrm{E}}>0.3$ for events which originally used the LH energy estimate).

2. The event must pass cuts to reject MIPs based on the event topology in the CAL and the energy deposited in the TKR.

3. The event must pass a set of cuts on the agreement between the TKR and CAL direction reconstruction.

4. The event must pass a tighter, energy-dependent, cut on $P_{\text {core: }}$

$$
P_{\text {core }}>\max \left(0.025,0.025+0.175\left(3.0-\log _{10}(E / 1 \mathrm{MeV})\right)\right. \text {. }
$$

5. The event must pass a tighter cut on $P_{\text {all }}$ :

$$
\begin{aligned}
P_{\text {all }}> & \max \left(0.7,0.996-1.4 \times 10^{-4}\right. \\
& \left.\times\left(\max \left(5.4-\log _{10}(E / 1 \mathrm{MeV}), 0\right)^{5.3}\right)\right) .
\end{aligned}
$$

As with the TRANSIENT event classes, it is worth noting that although the cuts are optimized somewhat differently in Pass 6, a similar series of cuts are applied to define the P6_DIFFUSE event class, which is roughly equivalent to the P7SOURCE selection.

\subsubsection{P7CLEAN Class Selection}

For the analysis of diffuse $\gamma$-ray emission, we need to reduce the background contamination to a level of about $\sim 0.1 \mathrm{~Hz}$ across the LAT FoV, to keep it below the extragalactic $\gamma$-ray background at all energies. For comparison, the total Galactic diffuse contribution is $\sim 1 \mathrm{~Hz}$, depending on where the LAT is pointing, though most of that is localized along the Galactic plane.

The selection of P7CLEAN class events starts from the P7SOURCE event class and includes the following additional cuts.

1. The event must pass a series of cuts designed to reject specific backgrounds such as CRs that passed through the mounting holes in the ACD, or the gaps along the corners of the ACD, with minimal costs to the $\gamma$-ray efficiency (see Section 4.2).

2. The event must pass cuts on the topology of the event in the CAL designed to remove hadronic CRs.

As with the two previous event classes, a similar series of cuts was applied to define the P6_DATACLEAN event class, which is roughly equivalent to the P7CLEAN event class.

\subsubsection{P7ULTRACLEAN Class Selection}

For the analysis of extragalactic diffuse $\gamma$-ray emission, we need to reduce the background contamination even further below the extragalactic $\gamma$-ray background rate to avoid introducing artificial spectral features. As illustrated in Figure 28, the residual contamination for the P7ULTRACLEAN class is $\sim 40 \%$ lower than that of the P7CLEAN class around $100 \mathrm{MeV}$ (the residual levels becoming more similar to each other as the energy increases and becoming the same above $10 \mathrm{GeV}$ ). 
Table 5

Publicly Released Event Selections and IRFs

\begin{tabular}{lccr}
\hline \hline Event Class & Pass 7 IRF Set & Pass 6 Counterpart & Pass 6 IRF Set \\
\hline P7TRANSIENT & P7TRANSIENT_V6 & P6_TRANSIENT & $\begin{array}{l}\text { P6_V1_TRANSIENT } \\
\text { P6_V3_TRANSIENT }\end{array}$ \\
\hline P7SOURCE & P7SOURCE_V6 ${ }^{\mathrm{a}}$ & P6_DIFFUSE & $\begin{array}{l}\text { P6_V1_DIFFUSE } \\
\text { P6_V3_DIFFUSE } \\
\text { P6_V11_DIFFUSE }\end{array}$ \\
\hline P7CLEAN & & & P6_V3_DATACLEAN \\
\hline P7ULTRACLEAN & P7CLEAN_V6 & & P7ULTRACLEAN_V6 \\
\hline
\end{tabular}

Notes. Note the slight change in naming conventions between Pass 6 and Pass 7. The Pass 7 naming convention emphasizes the point that we may release multiple IRFs for the same event class as we improve the IRFs and background rejection.

a We have also released P7SOURCE_V6MC and P7CLEAN_V6MC IRF sets, which feature an MC-based PSF that includes $\theta$ dependence that we have used when we need to minimize the potential of instrument-induced variability (see Section 6).

The selection of P7ULTRACLEAN class events is relatively simple, consisting of a tighter, energy-dependent cut on $P_{\text {all }}$ :

$$
\begin{gathered}
P_{\text {all }}>0.996-0.0394\left(\max \left(3.26-\log _{10}(\mathrm{E} / 1 \mathrm{MeV}), 0\right)^{1.78}\right) \\
(\text { Front }) \\
P_{\text {all }}>0.996-0.006\left(\max \left(4.0-\log _{10}(\mathrm{E} / 1 \mathrm{MeV}), 0\right)^{3.0}\right) \\
(\text { Back }) .
\end{gathered}
$$

\subsection{Publicly Released Data}

At the time of this writing, the LAT team has published results and released data for both the Pass 6 and Pass 7 event analyses, as well as several event classes and the associated IRFs for each iteration of the event analysis. Furthermore, as our understanding of the LAT has improved, we have updated the IRFs for particular event classes. Table 5 summarizes these data sets and associated IRFs. This paper will focus in particular on the performance of the P7SOURCE event class and the validation of the associated P7SOURCE_V6 IRFs since this is the data set the LAT team currently recommends for most analyses. Of the other Pass 7 event classes, P7TRANSIENT is recommended for the analysis of short $(<1000 \mathrm{~s})$ transient events such as GRBs, P7CLEAN is recommended for analyses requiring low CR background contamination, such as the study of large-scale diffuse emission, and P7ULTRACLEAN is recommended when CR background contamination must be minimized, even at the cost of some loss of effective area, such as when studying the extragalactic background. Accordingly, all plots, figures, and tables will be made with the P7SOURCE event sample and the P7SOURCE_V6 set of IRFs unless stated otherwise.

One other very important point is that the excellent stability of the LAT subsystems (see Section 2.1) means that changes in the instrument performance over time are not a consideration in defining the event analyses or IRFs. The small changes in performance at the subsystem level are easily addressed by calibrations applied during the event reconstruction procedure (Abdo et al. 2009a). Accordingly, to date, the LAT team is able to produce IRFs that are valid for the entire mission. This in turn simplifies the data analysis task by removing the need to split the LAT data by time range.

\subsection{Calibration Sources and Background Subtraction Methods}

Because of the complexity of the LAT and of the physics simulations of particle interactions we cannot expect the MC to perfectly reproduce the flight data. For this reason we have developed validation data sets for the IRFs. Although no astrophysical source has perfectly known properties, in practice there are several sources for which accurate background subtraction allows one to extract a clean $\gamma$-ray sample that we can use to validate the MC predictions. Table 6 summarizes these calibration sources and associated background subtraction techniques and MC samples. The remainder of the section discusses the particulars of these samples.

\subsubsection{The Vela Pulsar}

The Vela pulsar (PSR J0835-4510) has the largest integral flux $>100 \mathrm{MeV}$ of any $\gamma$-ray source, and has been well studied in the LAT energy range (Abdo et al. 2009e, 2010c). Furthermore, the pulsed nature of high-energy $\gamma$-ray emission gives us an independent control on the background. In fact, off-pulse $\gamma$-ray emission is almost entirely absent. These factors combine to make the Vela pulsar an almost ideal calibration source. Unfortunately, the spectrum of Vela cuts off at $\sim 3 \mathrm{GeV}$ and Vela is nearly undetectable above $30 \mathrm{GeV}$.

The selection criteria we use to define our Vela calibration samples are listed in Table 6. Specific calibration samples used for particular studies may include additional requirements. For example, the "P7TRANSIENT Vela calibration sample" includes all events in the P7TRANSIENT event class that pass the Vela calibration sample criteria. We used the TEMPO2 package ${ }^{76}$ (Hobbs et al. 2006) and a pulsar timing model ${ }^{77}$ derived from data taken with the Parkes radio telescope (Abdo et al. 2009e; Weltevrede et al. 2010) to assign a phase to each $\gamma$ ray.

We can achieve excellent statistical background subtraction for any distribution (i.e., spectrum, spatial distribution, any discriminating variable used in the event classification) by subtracting the distribution of off-pulse events (defined as phases in the range $[0.7,1.0])$ from the distribution of on-peak events (defined as phases in $[0.125,0.175] \cup[0.5125,0.6125]$ ). Figure 15 shows the distribution of phases of $\gamma$ rays in the P7TRANSIENT Vela calibration sample, including our standard on-peak and off-pulse regions. (Note that the PSF analysis in Section 6.2.1 uses slightly different definitions of on-peak $([0.12,0.17] \cup[0.52,0.57])$ and off-pulse $[0.8,1.0]$ regions; the

\footnotetext{
76 http://www.atnf.csiro.au/research/pulsar/tempo2/

77 http://fermi.gsfc.nasa.gov/ssc/data/access/lat/ephems/
} 
Table 6

Summary Table of Calibration Data Samples

\begin{tabular}{|c|c|c|c|c|}
\hline Parameter & Vela Pulsar (Section 3.6.1) & AGN (Section 3.6.2) & Earth Limb (Section 3.6.3) & Galactic Ridge (Section 3.6.4) \\
\hline Mission elapsed time (MET) & 239414402-302486402 & 239414402-302486402 & $237783738-239102693,244395837-244401823$ & 239414402-302486402 \\
\hline Energy range & $30 \mathrm{MeV}-10 \mathrm{GeV}$ & $1-100 \mathrm{GeV}$ & $10-100 \mathrm{GeV}$ & $17 \mathrm{MeV}-300 \mathrm{GeV}$ \\
\hline Selection & $15^{\circ}$ region of interest $(\mathrm{ROI})$ around Vela & $6^{\circ} \mathrm{ROI}$ around each of the $\mathrm{AGNs}^{\mathrm{a}}$ & Zenith cut & Clean and dirty regions (Section 3.6.4) \\
\hline Zenith cut & $\theta_{z}<100^{\circ \mathrm{b}}$ & $\theta_{z}<100^{\circ}$ & $105^{\circ}<\theta_{z}<120^{\circ}$ & $\theta_{z}<100^{\circ}$ \\
\hline Rocking angle $\mathrm{cut}^{\mathrm{c}}$ & Yes & Yes & No & Yes \\
\hline Data quality cut ${ }^{\mathrm{d}}$ & Yes & Yes & Yes & Yes \\
\hline Bkg. subtraction & Phase-gated & Angular separation $\alpha$ to the nearest AGN & Zenith angle $\theta_{z}$ & Galactic latitude $^{\mathrm{e}}$ \\
\hline Signal region & $\phi \in[0.125,0.175], \phi \in[0.5125,0.6125]$ & $\alpha<0.5$ & $111.10<\theta_{z}<112.95$ & Clean region \\
\hline Background region & $\phi \in[0.7,1]$ & $3.87288<\alpha<4^{\circ}$ & $108.66<\theta_{z}<109.57,114.52<\theta_{z}<115.47$ & Dirty region \\
\hline MC sample & $\theta$-weighted allGamma & $\theta$-weighted allGamma & Limb flux model & None \\
\hline
\end{tabular}

Notes.

a The criteria used to select the AGN sample are described in Section 3.6.2.

${ }^{\mathrm{b}}$ The zenith angle cut for the Vela sample is applied over the entire $15^{\circ} \mathrm{ROI}$

${ }^{c}$ Standard rocking angle selection ABS (ROCK_ANGLE) $<52$ in gtmktime.

d Standard data quality selection DATA_QUAL $==1$ \&\& LAT_CONFIG $==1$ in gtmktime.

${ }^{\mathrm{e}}$ In the case of the Galactic ridge, we cannot subtract background accurately enough for detailed quantitative validations; however, we can distinguish between regions of higher and lower $\gamma$-ray-to-CR ratios. 


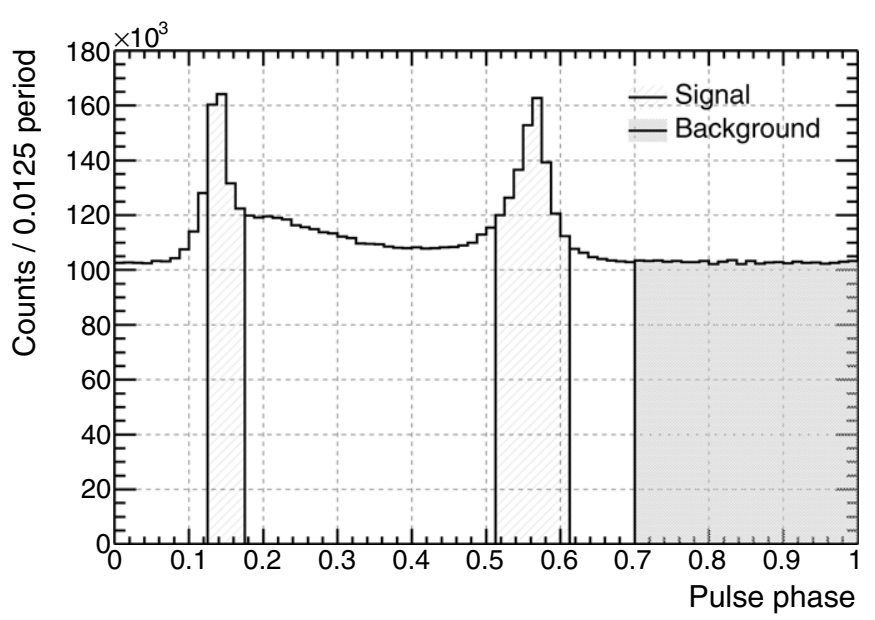

Figure 15. Phase-folded times for events in the P7TRANSIENT Vela calibration sample, which includes events between $30 \mathrm{MeV}$ and $10 \mathrm{GeV}$. The signal and background regions, as defined in the background subtraction analysis, are highlighted.

slight difference in definition causes no significant change in the results.)

Admittedly, all bright pulsars are potential candidates; nonetheless, for this analysis the consequent increase in event statistics does not warrant the procedural complication required to deal with a stacked sample of pulsars (see also Section 6.2).

\subsubsection{Bright Active Galactic Nuclei}

At $1 \mathrm{GeV}$ the $95 \%$ containment radius for the P7SOURCE event class is $\sim 1$. 4 for front-converting events and $\sim 2.5$ for backconverting events (see Section 6). Given the density of bright $\gamma$-ray sources in the sky, above this energy the instrument PSF becomes narrow enough that we can use the angular distance $\alpha$ between a $\gamma$ ray and the nearest celestial $\gamma$-ray source as a good discriminator for background subtraction, particularly at high Galactic latitudes where there are fewer sources and the interstellar diffuse emission is less pronounced. Unfortunately, no single source is bright enough to provide adequate statistics to serve as a good calibrator. However, by considering $\gamma$ rays from a sample of bright and/or hard spectrum active galactic nuclei (AGNs) that are isolated from other hard sources, we can create a good calibration sample for energies from $1 \mathrm{GeV}$ to $100 \mathrm{GeV}$.

Table 7 lists the AGNs that we use here, Figure 16 shows their positions, and Figure 17 shows a comparison of their spectral index $(\Gamma)$ and integral $\gamma$-ray flux between $1 \mathrm{GeV}$ and $100 \mathrm{GeV}\left(F_{35}\right)$ to other AGNs in the second LAT source catalog (2FGL; Nolan et al. 2012). Note that different but overlapping sets of AGNs are used for $A_{\text {eff- }}$ and PSF-related studies. More information about the source properties can be found in Abdo et al. (2010b) and Nolan et al. (2012).

The selection criteria we use to define AGN calibration samples are listed in Table 6. As with the Vela calibration samples, specific calibration samples used for particular studies may include additional requirements (e.g., the P7TRANSIENT AGN calibration sample).

To use this calibration sample to perform background subtraction, we define signal and background regions in terms of the angular distance $\alpha$ between the $\gamma$ ray and the closest AGN. Specifically, we use $\alpha<0.5$ for the signal region and the annulus $3.87288<\alpha<4^{\circ}$ for the background region. These ranges are chosen so that the background region contains four

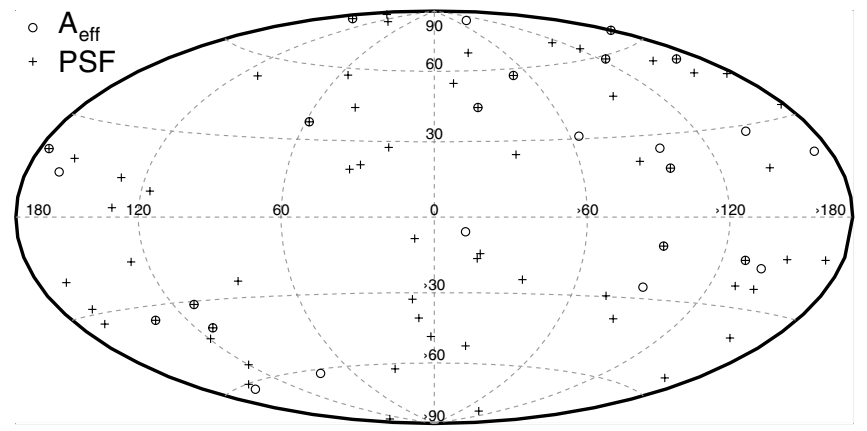

Figure 16. Positions of the AGNs in the calibration samples, shown in a Hammer-Aitoff projection in Galactic coordinates. Circles mark the AGNs used for $A_{\text {eff }}$ studies and crosses indicate those used in PSF studies.

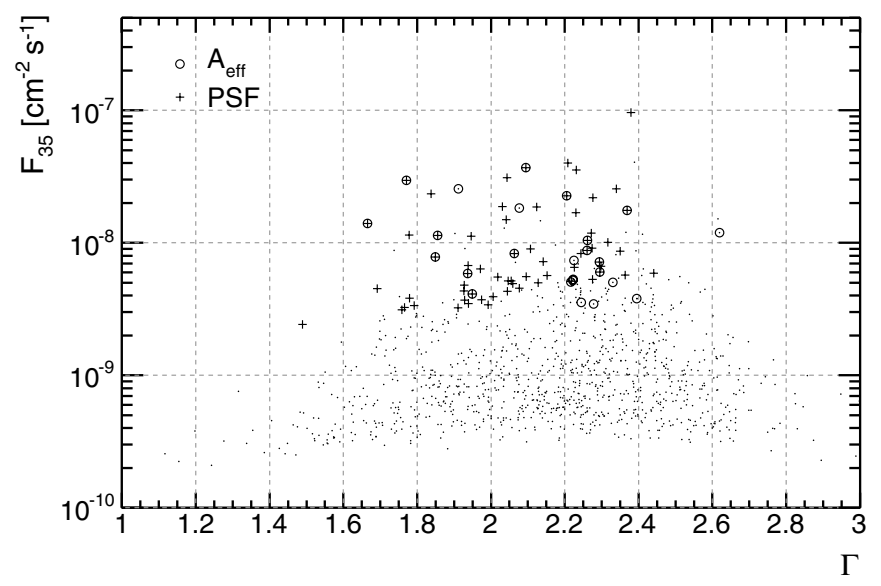

Figure 17. Integral $\gamma$-ray fluxes between $1 \mathrm{GeV}$ and $100 \mathrm{GeV}\left(F_{35}\right)$ and spectral indices $(\Gamma)$ of the AGNs in the calibration samples. Circles mark the AGNs used for $A_{\text {eff }}$ studies and crosses indicate those used in PSF studies. The dots mark all the other sources associated with AGNs in the 2FGL catalog.

times the solid angle of the signal region. We adopted the separation between the signal and background regions in order to minimize signal $\gamma$ rays from the tails of the PSF leaking into the background region. Figure 18 shows the squares of the angular separations between $\gamma$ rays and the nearest bright AGN for all $\gamma$ rays in the P7TRANSIENT event class in $6^{\circ}$ regions around the 25 AGNs listed as the $A_{\text {eff }}$ calibration sample in Table 7, including our definitions of source and background regions.

\subsubsection{The Earth Limb}

The Earth's atmosphere is a very bright $\gamma$-ray source. Furthermore, at energies above a few GeV the $\gamma$-ray flux seen by the LAT is dominated by $\gamma$ rays from the interactions of primary CR protons with the upper atmosphere. This consideration, together with the narrowness of the PSF at energies $>10 \mathrm{GeV}$, causes the Earth limb to appear as a very bright and sharp feature, which provides an excellent calibration source. Furthermore, we have selected data from 200 orbits during which the LAT was pointed near the Earth limb as the basis of the Earth limb calibration sample. The selection criteria we use to define the Earth limb calibration samples are listed in Table 6. When using the Earth limb as a calibration source we generally limit the energy range to energies $>10 \mathrm{GeV}$, primarily because at lower energies orbital variations in the geomagnetic field significantly affect the $\gamma$-ray fluxes (however see Section 7.5 for a counterexample).

For this calibration source, we define our signal region as $111.1002<\theta_{z}<112.9545$ and background regions just above and below the limb: $108.6629<\theta_{z}<109.5725$ and 
Table 7

List of the AGNs Used in the AGN Calibration Samples for $A_{\text {eff }}$ and PSF Studies

\begin{tabular}{|c|c|c|c|c|}
\hline Source & $\begin{array}{c}l \\
\left(^{\circ}\right)\end{array}$ & $\begin{array}{c}b \\
\left({ }^{\circ}\right)\end{array}$ & 2FGL & Used \\
\hline KUV 00311-1938 & 94.17 & -81.21 & J0033.5-1921 & PSF \\
\hline PKS 0118-272 & 213.58 & -83.53 & J0120.4-2700 & PSF \\
\hline B3 $0133+388$ & 132.43 & -22.95 & J0136.5+3905 & PSF \\
\hline OC 457 & 130.79 & -14.32 & J0136.9+4751 & $A_{\text {eff }}$ PSF \\
\hline PKS 0208-512 & 276.12 & -61.76 & J0210.7-5102 & $A_{\text {eff }}$ \\
\hline PKS 0215+015 & 162.20 & -54.41 & J0217.9+0143 & PSF \\
\hline S4 0218+35 & 142.60 & -23.49 & $\mathrm{~J} 0221.0+3555$ & PSF \\
\hline $3 \mathrm{C} 66 \mathrm{~A}$ & 140.14 & -16.77 & $\mathrm{~J} 0222.6+4302$ & $A_{\text {eff }}$ \\
\hline $\mathrm{AO} 0235+164$ & 156.78 & -39.10 & J0238.7+1637 & PSF \\
\hline PKS 0250-225 & 209.72 & -62.10 & J0252.7-2218 & $A_{\text {eff }}$ \\
\hline PKS 0301-243 & 214.64 & -60.17 & J0303.4-2407 & PSF \\
\hline NGC 1275 & 150.59 & -13.25 & J0319.8+4130 & PSF \\
\hline PMN J0334-3725 & 240.22 & -54.36 & J0334.3-3728 & PSF \\
\hline PKS 0420-01 & 195.28 & -33.15 & $\mathrm{~J} 0423.2-0120$ & PSF \\
\hline PKS 0426-380 & 240.70 & -43.62 & J0428.6-3756 & PSF \\
\hline MG2 J043337+2905 & 170.52 & -12.62 & J0433.5+2905 & PSF \\
\hline PKS 0440-00 & 197.21 & -28.44 & J0442.7-0017 & PSF \\
\hline PKS 0447-439 & 248.81 & -39.91 & J0449.4-4350 & $A_{\text {eff }}$ PSF \\
\hline PKS 0454-234 & 223.73 & -34.90 & J0457.0-2325 & $A_{\text {eff }}$ PSF \\
\hline 1ES 0502+675 & 143.80 & 15.90 & J0508.0+6737 & PSF \\
\hline TXS 0506+056 & 195.40 & -19.62 & J0509.4+0542 & PSF \\
\hline PKS 0537-441 & 250.08 & -31.09 & J0538.8-4405 & $A_{\text {eff }}$ PSF \\
\hline TXS 0628-240 & 232.68 & -15.00 & J0630.9-2406 & PSF \\
\hline B3 $0650+453$ & 171.20 & 19.36 & J0654.2+4514 & $A_{\text {eff }}$ \\
\hline PKS 0700-661 & 276.77 & -23.78 & J0700.3-6611 & PSF \\
\hline MG2 J071354+1934 & 197.68 & 13.61 & J0714.0+1933 & $A_{\text {eff }}$ \\
\hline B2 $0716+33$ & 185.06 & 19.85 & J0719.3+3306 & $A_{\mathrm{eff}} \mathrm{PSF}$ \\
\hline S5 $0716+71$ & 143.97 & 28.02 & $\mathrm{~J} 0721.9+7120$ & $A_{\mathrm{eff}}$ \\
\hline PKS 0727-11 & 227.77 & 3.13 & $\mathrm{~J} 0730.2-1141$ & PSF \\
\hline PKS 0735+17 & 201.85 & 18.06 & $\mathrm{~J} 0738.0+1742$ & PSF \\
\hline PKS 0805-07 & 229.04 & 13.16 & J0808.2-0750 & PSF \\
\hline S4 0814+42 & 178.21 & 33.41 & J0818.2+4223 & PSF \\
\hline PKS 0823-223 & 243.97 & 8.92 & J0825.9-2229 & PSF \\
\hline S4 0917+44 & 175.70 & 44.81 & J0920.9+4441 & PSF \\
\hline $4 C+55.17$ & 158.59 & 47.94 & J0957.7+5522 & PSF \\
\hline 1H $1013+498$ & 165.53 & 52.73 & $\mathrm{~J} 1015.1+4925$ & $A_{\text {eff }}$ PSF \\
\hline $4 \mathrm{C}+01.28$ & 251.50 & 52.77 & $\mathrm{~J} 1058.4+0133$ & PSF \\
\hline TXS $1055+567$ & 149.57 & 54.42 & $\mathrm{~J} 1058.6+5628$ & PSF \\
\hline Mkn 421 & 179.82 & 65.03 & $\mathrm{~J} 1104.4+3812$ & $A_{\mathrm{eff}} \mathrm{PSF}$ \\
\hline Ton 599 & 199.41 & 78.37 & J1159.5+2914 & $A_{\text {eff }}$ PSF \\
\hline W Comae & 201.69 & 83.28 & $\mathrm{~J} 1221.4+2814$ & PSF \\
\hline $4 C+21.35$ & 255.07 & 81.66 & $\mathrm{~J} 1224.9+2122$ & PSF \\
\hline PKS 1244-255 & 301.60 & 37.08 & J1246.7-2546 & $A_{\text {eff }}$ PSF \\
\hline PG 1246+586 & 123.74 & 58.77 & $\mathrm{~J} 1248.2+5820$ & $A_{\text {eff }}$ PSF \\
\hline S4 1250+53 & 122.36 & 64.08 & $\mathrm{~J} 1253.1+5302$ & PSF \\
\hline $3 C 279$ & 305.10 & 57.06 & $\mathrm{~J} 1256.1-0547$ & PSF \\
\hline OP 313 & 85.59 & 83.29 & $\mathrm{~J} 1310.6+3222$ & $A_{\text {eff }}$ \\
\hline GB $1310+487$ & 113.32 & 68.25 & $\mathrm{~J} 1312.8+4828$ & PSF \\
\hline PMN J1344-1723 & 320.48 & 43.67 & $\mathrm{~J} 1344.2-1723$ & PSF \\
\hline PKS $1424+240$ & 29.48 & 68.20 & $\mathrm{~J} 1427.0+2347$ & PSF \\
\hline PKS 1440-389 & 325.64 & 18.72 & J1443.9-3908 & PSF \\
\hline PKS 1454-354 & 329.89 & 20.52 & $\mathrm{~J} 1457.4-3540$ & PSF \\
\hline PKS 1502+106 & 11.37 & 54.58 & $\mathrm{~J} 1504.3+1029$ & PSF \\
\hline AP Librae & 340.70 & 27.58 & $\mathrm{~J} 1517.7-2421$ & PSF \\
\hline B2 $1520+31$ & 50.18 & 57.02 & $\mathrm{~J} 1522.1+3144$ & $A_{\text {eff }}$ PSF \\
\hline GB6 J1542+6129 & 95.38 & 45.40 & $\mathrm{~J} 1542.9+6129$ & PSF \\
\hline PG $1553+113$ & 21.92 & 43.95 & $\mathrm{~J} 1555.7+1111$ & $A_{\text {eff }}$ PSF \\
\hline $1 \mathrm{H} 1720+117$ & 34.11 & 24.47 & $\mathrm{~J} 1725.0+1151$ & PSF \\
\hline B2 $1732+38$ A & 64.04 & 31.02 & $\mathrm{~J} 1734.3+3858$ & $A_{\text {eff }}$ \\
\hline PMN J1802-3940 & 352.44 & -8.42 & J1802.6-3940 & PSF \\
\hline PKS 1830-211 & 12.15 & -5.72 & J1833.6-2104 & $A_{\text {eff }}$ \\
\hline S4 1849+67 & 97.50 & 25.03 & J1849.4+6706 & $A_{\text {eff }}$ \\
\hline TXS $1902+556$ & 85.96 & 20.51 & J1903.3+5539 & PSF \\
\hline 1H 1914-194 & 18.24 & -14.30 & J1917.6-1921 & PSF \\
\hline TXS 1920-211 & 17.17 & -16.26 & $\mathrm{~J} 1923.5-2105$ & PSF \\
\hline
\end{tabular}


Table 7

(Continued)

\begin{tabular}{|c|c|c|c|c|}
\hline Source & $\begin{array}{c}l \\
\left({ }^{\circ}\right)\end{array}$ & $\begin{array}{c}b \\
\left({ }^{\circ}\right)\end{array}$ & 2FGL & Used \\
\hline 1ES $1959+650$ & 98.02 & 17.67 & $\mathrm{~J} 2000.0+6509$ & $A_{\text {eff }}$ PSF \\
\hline PKS 2005-489 & 350.37 & -32.61 & $\mathrm{~J} 2009.5-4850$ & PSF \\
\hline PKS 2023-07 & 36.89 & -24.39 & J2025.6-0736 & PSF \\
\hline PKS 2052-47 & 352.58 & -40.38 & $\mathrm{~J} 2056.2-4715$ & PSF \\
\hline MH 2136-428 & 358.29 & -48.32 & $\mathrm{~J} 2139.3-4236$ & PSF \\
\hline PKS 2155-304 & 17.74 & -52.24 & $\mathrm{~J} 2158.8-3013$ & PSF \\
\hline BL Lacertae & 92.60 & -10.46 & $\mathrm{~J} 2202.8+4216$ & $A_{\text {eff }}$ PSF \\
\hline PKS 2201+171 & 75.68 & -29.63 & $\mathrm{~J} 2203.4+1726$ & PSF \\
\hline B2 2234+28A & 90.12 & -25.66 & $\mathrm{~J} 2236.4+2828$ & $A_{\text {eff }}$ \\
\hline $3 \mathrm{C} 454.3$ & 86.12 & -38.18 & $\mathrm{~J} 2253.9+1609$ & PSF \\
\hline PKS 2326-502 & 332.00 & -62.30 & $\mathrm{~J} 2329.2-4956$ & PSF \\
\hline
\end{tabular}

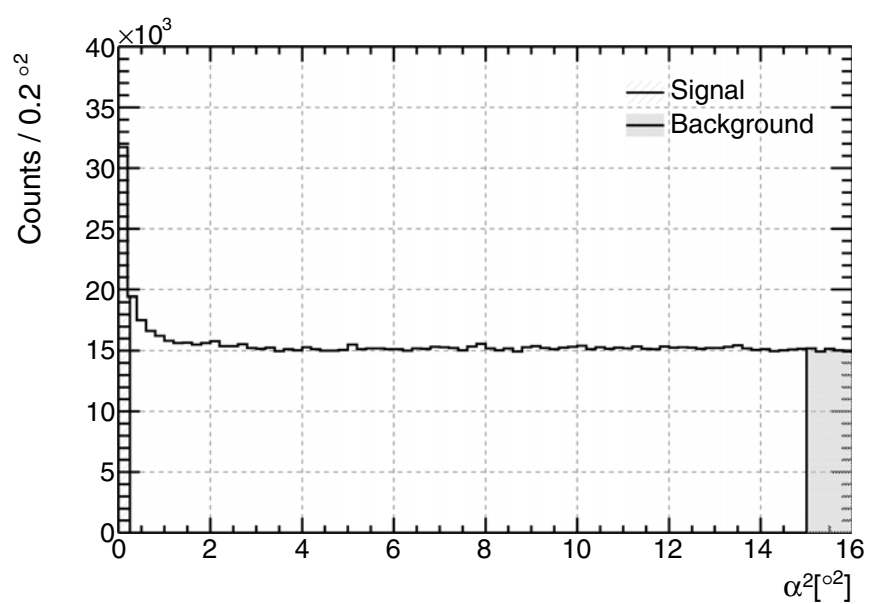

Figure 18. Square of the angular separation between reconstructed $\gamma$-ray directions and the AGNs for events in the P7TRANSIENT AGN calibration sample, which includes $\gamma$ rays in the energy range $1-100 \mathrm{GeV}$. The signal and background regions, as defined in the background subtraction analysis, are highlighted.

$114.5193<\theta_{z}<115.4675$. Note that these ranges are defined to give the same solid angle $(0.06 \pi$ sr $)$ in the signal and background regions.

Figure 19 shows the zenith angle distribution for all $\gamma$ rays in the P7TRANSIENT event class for the Earth limb calibration sample.

\subsubsection{The Galactic Ridge}

At energies above $\sim 30 \mathrm{GeV}$, no single source provides enough $\gamma$ rays for a good comparison between flight data and MC. However, the combination of bright Galactic sources and Galactic diffuse backgrounds means that there is a very large excess of $\gamma$ rays coming from the Galactic plane relative to high Galactic latitudes.

The intensity of the $\gamma$-ray emission at low latitudes in the inner Galaxy is more than an order of magnitude greater than at high latitudes in the outer Galaxy. In contrast, the intensity of the CR background is approximately isotropic for observation periods longer than the 53.4 day orbital precession period.

Unfortunately, since the Galaxy extends over much of the sky, and since the data set consists of several thousand orbits it is not practical to disentangle the variations of exposure from the spatial variations in Galactic diffuse emission without relying on detailed modeling of the Galactic diffuse emission. Accordingly, we use the Galactic ridge primarily when we are developing our

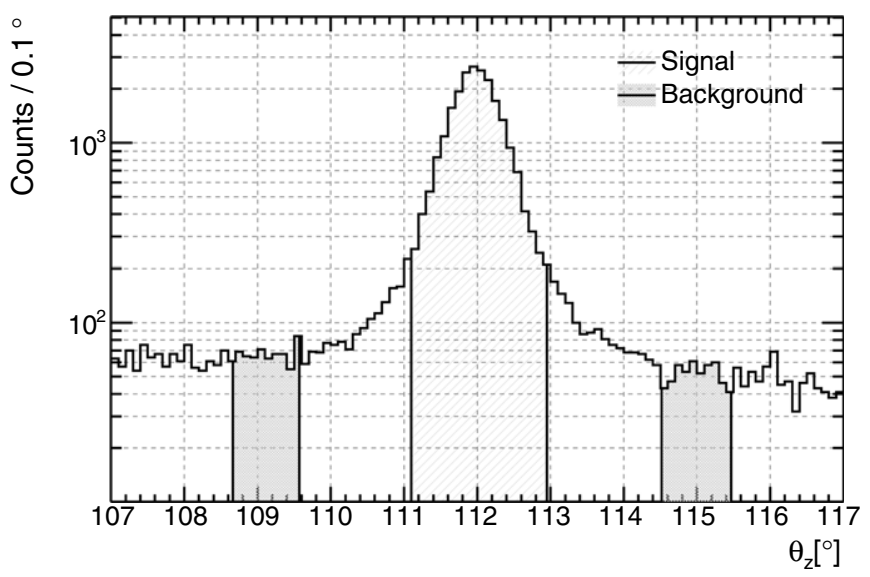

Figure 19. Angle with respect to the zenith for events in the P7TRANSIENT Earth limb calibration sample, which includes $\gamma$ rays with energies above $10 \mathrm{GeV}$. The signal and background regions, as defined in the background subtraction analysis, are highlighted.

event selections, rather than for precise calibration of the LAT performance.

Specifically, we developed the event classes that require a high $\gamma$-ray purity, i.e., the event classes used in the analysis of celestial point sources and diffuse emission (see Section 3.4), in part by tuning our selection criteria to maximize the contrast between regions in the bright Galactic plane and at high Galactic latitudes. This helped to mitigate the risk that insufficient statistics in the MC training sample or limited accuracy of the $\mathrm{MC}$ description of the geometry of the detector and the particle interactions in the LAT limited the discriminating power and accuracy of the event classification analysis.

The selection criteria we use to define the Galactic ridge calibration samples are listed in Table 6. In particular, we use the region $\left(|b|<1.5,-40^{\circ}<l<50^{\circ}\right.$, which was selected to maximize the total $\gamma$-ray flux) to define a clean data sample and the region $\left(|b|>50^{\circ}, 90^{\circ}<l<270^{\circ}\right)$ to define a dirty data sample. The ratio of $\gamma$ rays to $\mathrm{CR}$ background in the clean region is more than an order of magnitude higher than the same ratio in the dirty region. Figure 20 shows the count maps for the P7SOURCE samples for both regions.

Furthermore, to give a sense of the statistics of these samples at high energies, Figure 21 shows the Galactic latitude distribution for all $\gamma$ rays in the P7TRANSIENT event class with energies above $17783 \mathrm{MeV}$ (i.e., $\left.\log _{10}(E / 1 \mathrm{MeV})>4.25\right)$. 


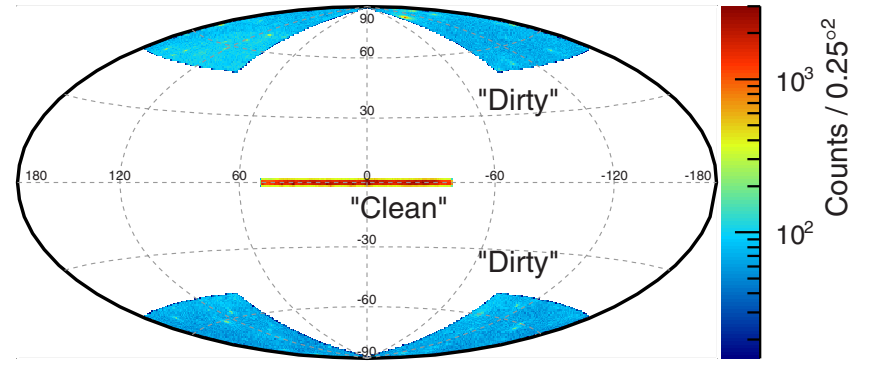

Figure 20. Definitions of the clean and dirty regions, showing the counts in both regions in a Hammer-Aitoff projection. This figure uses the data in the P7SOURCE Galactic ridge calibration sample.

(A color version of this figure is available in the online journal.)

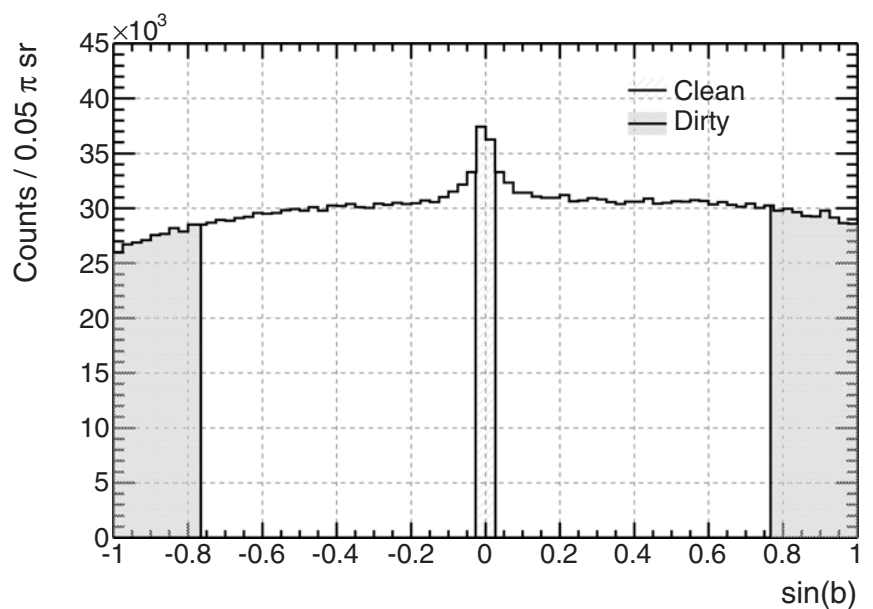

Figure 21. Sine of Galactic latitude for events above $17,783 \mathrm{MeV}$ in the P7TRANSIENT Galactic ridge calibration sample. The Galactic latitude selections for the clean and dirty regions are highlighted. Note that the definition of the clean and dirty regions also include selections on Galactic longitude.

\subsubsection{Summary of Astrophysical Calibration Sources}

As we will see in the next sections, the IRFs depend heavily on $\theta$ (and, to a lesser extent, on $\phi$ ). Therefore, any detailed comparison between flight data and $\mathrm{MC}$ simulations must account for the distribution of observing profile, particularly $t_{\mathrm{obs}}(\theta)$. How best to account for the observing profile depends on the particulars of the calibration samples.

For any point source, the observing profile is determined by the position of the source, the rocking angle of the LAT and the amount of time spent in survey mode relative to pointed observations. Figure 22 shows the observing profile for Vela for the first two years of the mission. Rather than produce a dedicated large statistics MC sample for Vela, we re-use our allGamma MC sample, re-weighting the events in that sample so as to match the Vela observing profile.

Similarly, we re-weight the allGamma MC to match the summed observing profiles of all of the AGNs of our sample, which is shown in Figure 23. Unfortunately, since AGNs are intrinsically variable, and since the AGNs in this sample span a range of fluxes, this re-weighting technique will not work as well with this sample. On the other hand, by taking a large set of AGNs, we reduce the bias due to the variability of any one particular source. In broad terms, our re-weighted MC sample reproduced the $\theta$ distribution of the AGN sample to better than $2 \%$ (see Section 5.5). Finally, we note that since the PSF is narrower above $1 \mathrm{GeV}$, and the ROI around each AGN is only

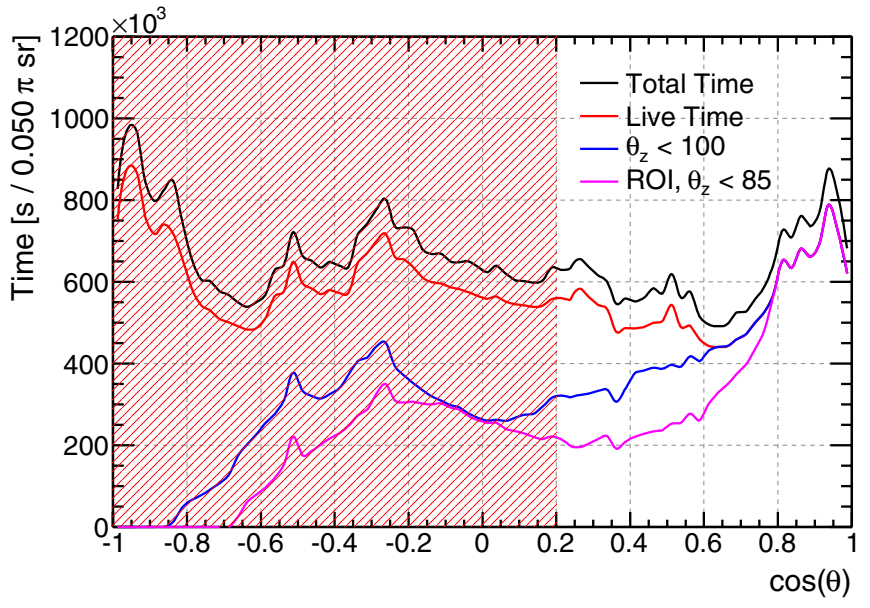

Figure 22. Vela observing profile: starting from the top, the curves show the accumulated time as a function of $\cos \theta$ for the first two years of the mission, the accumulated observing time (accounting for the dead time when the LAT triggers), the time during which Vela was less than $100^{\circ}$ from the zenith, and the time during which the entire $15^{\circ} \mathrm{ROI}$ around Vela was less than $100^{\circ}$ from the zenith (or, equivalently, that Vela was less than $85^{\circ}$ from the zenith). The shaded region corresponds to the area outside the LAT FoV.

(A color version of this figure is available in the online journal.)

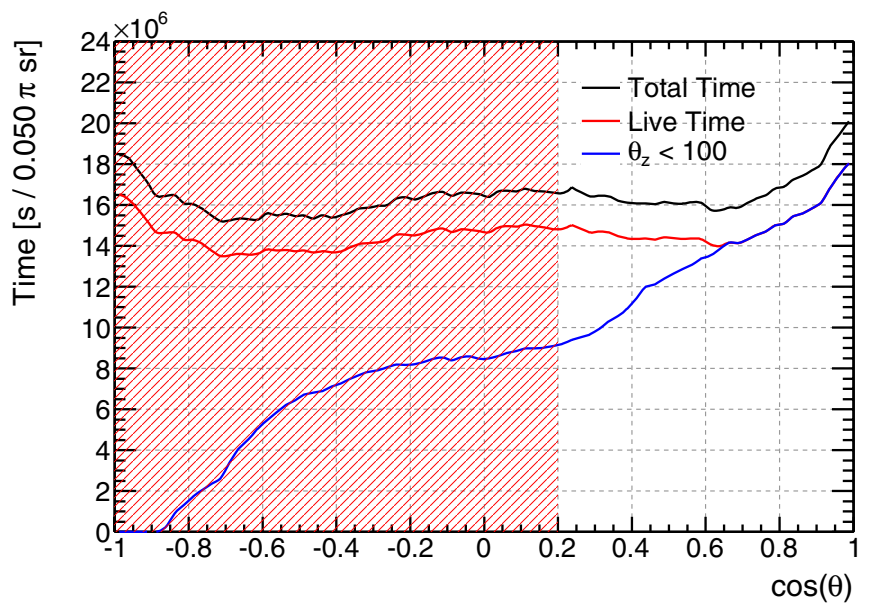

Figure 23. Sum of the observing profiles for the AGN sample: starting from the top, the curves show the sum of the accumulated time as a function of $\cos \theta$ for the first two years of the mission, the sum of the accumulated live time, and the sum of the accumulated live times during which each AGN was less than $100^{\circ}$ from the zenith. The shaded region corresponds to the area outside the LAT FoV.

(A color version of this figure is available in the online journal.)

$6^{\circ}$ we do not apply the ROI-based $\theta_{z}$ cut when building the AGN calibration samples.

Since the Earth limb is a spatially extended source, we cannot apply the re-weighting technique we used for the Vela and AGN samples to account for the observing profile. On the other hand, since the data set consists of only 200 orbits, and the Earth limb emission is well understood above $10 \mathrm{GeV}$ we can produce an MC simulation of the Earth limb emission for those orbits and compare it with the flight data (see Section 2.5.2).

Finally, Figure 24 shows the statistics available for each of the samples. This shows that the calibration sources span most of the LAT energy range, certainly from $30 \mathrm{MeV}$ up to at least $100 \mathrm{GeV}$. 


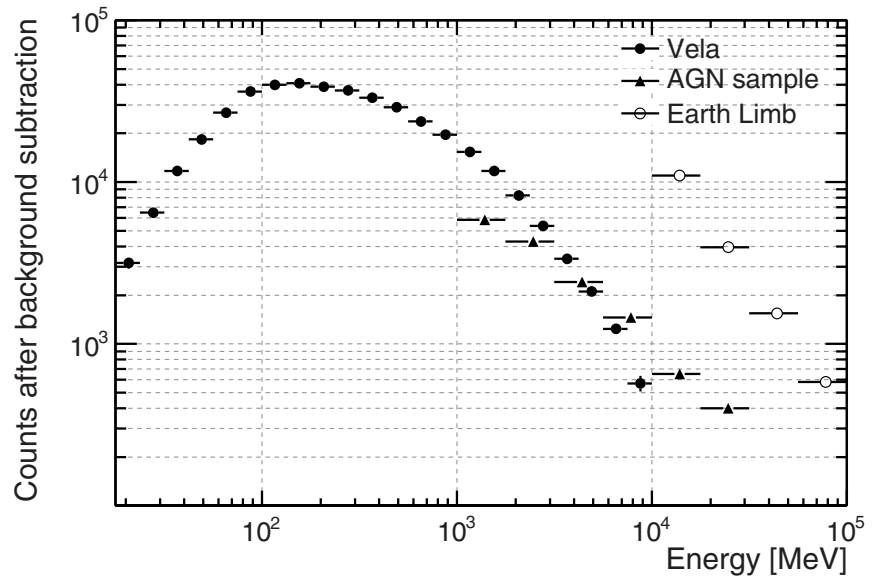

Figure 24. Excess counts in the signal regions as a function of energy for Vela, stacked AGN, and Earth limb calibration samples for the P7TRANSIENT event class. The horizontal error bars indicate the energy binning used with the different samples.

\section{BACKGROUND CONTAMINATION}

In this section we discuss the residual particle backgrounds, methods to estimate the contamination of LAT $\gamma$-ray samples by these backgrounds and how to treat such contamination in highlevel analysis. In this context, we define particle backgrounds as all events that are classified as $\gamma$ rays in a given LAT event class but originate from CRs or the interactions of CRs in the Earth's atmosphere. Therefore, the particle backgrounds include both charged and neutral particles-including secondary $\gamma$ rays.

The LAT background model is described in detail in Section 2.5.4. We focus here on describing the particle background contamination in the high-purity event classes, i.e., the ones used for single source, source population, and diffuse emission analysis (P7SOURCE and above). We also focus on average background contamination for long (few months or longer) observation periods. The CR-induced particle background is extremely variable throughout the orbit of the LAT; therefore, estimates of particle backgrounds for brief transient sources must be derived from a dedicated analysis of the periods of interest (e.g., as done in Abdo et al. 2009d).

\subsection{Residual Background Contamination in Monte Carlo Simulations}

We can estimate the residual background in the various event classes by propagating the LAT background model (see Section 2.5.4) through the full Geant4-based LAT detector simulation chain and applying the event classification analysis on the simulated data (see Sections 2.2, 2.4, and 2.5).

In comparison to the pre-launch particle background model shown in Figure 10, we have implemented substantial improvements in our model of the primary $\mathrm{CR}$ protons and electrons. The effects of the geomagnetic cutoff on the directional and energy dependence of the primary CR flux in the pre-launch were based on a dipole approximation of the geomagnetic field. Currently, we simulate an isotropic flux of CRs, and trace the trajectory of each particle backward through the geomagnetic magnetic field, eliminating any particles that intersect the Earth or lower atmosphere. We are using the current version of the International Geomagnetic Reference Field (IGRF-11), a high-order multipole model of the geomagnetic field (Finlay et al. 2010), and the publicly available trajectory tracing code described in Smart \& Shea (2005). We oversample the primary proton and electron spectrum at high energies to obtain sufficient statistics up to $\sim 600 \mathrm{GeV}$ in reconstructed energy, and obtain rate predictions by appropriately deweighting events at the oversampled energies.

A total of $2.2 \times 10^{12}$ primary protons and $1.6 \times 10^{8}$ primary electrons were generated. In addition, the equivalent of $80 \mathrm{ks}$ ( $\sim 1$ day) of instrument live time of background events from CR secondaries produced in the Earth atmosphere were simulated according to the spatial and spectral distributions in the prelaunch LAT particle background model (i.e., with no trajectory tracing). This intensive simulation effort was used to determine the CR-induced background between $100 \mathrm{MeV}$ and $\sim 600 \mathrm{GeV}$ in the P7SOURCE, P7CLEAN, and P7ULTRACLEAN event classes.

However, these simulations still have important shortcomings. For example, the modeling of inelastic interactions of alpha particles and heavier ions does not match our observations (see further discussion in Section 4.2, especially Figure 25(b)). The particle background model for secondaries produced in CR interactions in the atmosphere is derived from measurements from satellites with different orbits and during different parts of
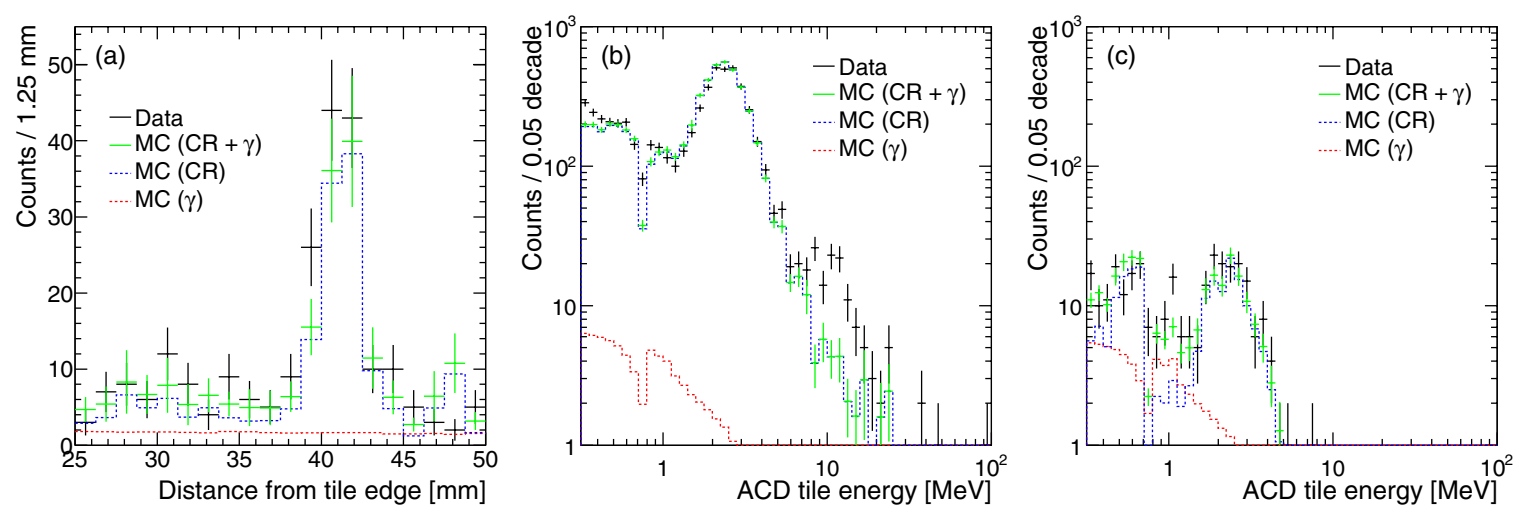

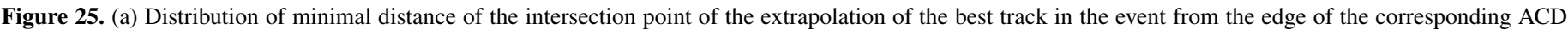

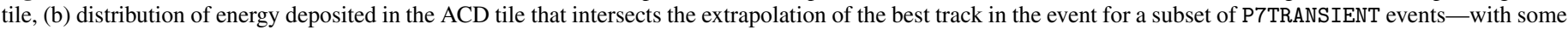

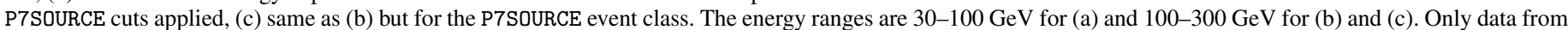

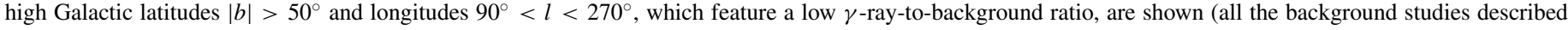

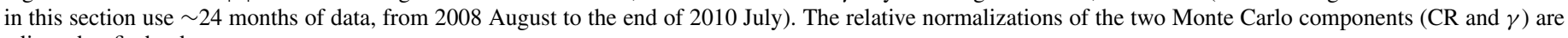
adjusted to fit the data.

(A color version of this figure is available in the online journal.) 
the solar cycle, and has been projected to the LAT orbit. Furthermore, due to our required background suppression factors of up to $10^{6}$, even small inaccuracies in our simulation of particle interactions with the LAT can potentially lead to large discrepancies between predicted and actual charged particle background rates.

As a consequence, we take several measures to account for any such shortcomings and minimize possible discrepancies. First, we compare a region on the sky with a high $\gamma$-rayto-residual-background ratio to a region with a low $\gamma$-ray-toresidual background ratio to isolate those contributions caused by the accumulation of background in the latter data set; and we compare those contributions to the predictions from the background simulations (see Section 4.2). Second, we compare several key event parameters between data and simulation for high-purity $\gamma$-ray samples to crosscheck the agreement between data and simulation, and we slightly adjust the intensities of our CR background model based on a fit of the shapes of two key event classification variables with signal and background component (see Section 4.3).

\subsection{Estimating and Reducing Residual Background Using Test Samples}

We already described the usage of data samples with different $\gamma$-ray-to-background ratios to define high-purity event classes in Section 3.6.4, and we detailed the resulting event classification cuts in Sections 3.4.3-3.4.5. A key part of the development of the event classes was using the same clean and dirty samples to search for and eliminate residual background that is either not simulated with sufficient accuracy or has passed the multivariate event selection that was trained on a limited-statistics sample of simulated events.

Two specific examples of such improvements to the event classification are described in this section. The first is designed to mitigate ACD inefficiencies around the mounting holes of the ACD tiles. Figure 25(a) shows the distribution of the closest distance of the extrapolation of the best track in the event from the edge of an ACD tile. Only events that are classified as $\gamma$ rays in the P7SOURCE class are shown. A peak starting $39 \mathrm{~mm}$ from the edge is clearly visible, corresponding to the closest distance of many mounting holes in the ACD from the edge of a tile, where charged particles entering the LAT often leave very small or undetectable signals due to inefficiencies in the ACD response near these mounting holes. A matching peak is visible in the simulation, showing a good example of the detailed description of the LAT detector model entering the MC simulation of the LAT. This particular source of residual background has not been removed by the event classification scheme but can be easily eliminated. For the P7CLEAN and P7UTLTRACLEAN classes we remove all events where the best track extrapolates to a range between 35 and $45 \mathrm{~mm}$ from the closest edge of an ACD tile that additionally produces a signal in the first TKR layer (see Section 3.4.4).

The second example demonstrates the removal of effects from poorly simulated interactions like the inelastic interactions of alpha particles and heavier nuclei. Figure 25(b) shows the distribution of the energy deposited in the ACD tile closest to the extrapolation of the best track in the event onto the ACD plane. The events included pass the P7TRANSIENT selection and additionally some of the cuts used to define the P7SOURCE class (omitting cuts that are effective for reducing heavy nuclei contamination). A peak at a few $\mathrm{MeV}$ is clearly visible in both data and simulation corresponding to residual protons traversing the ACD. The second peak above $10 \mathrm{MeV}$, corresponding to residual helium, is almost completely missing in the simulation. Figure 25(c) shows the same distribution after all selection criteria for the P7SOURCE class have been applied. The residual helium peak has been removed and data and simulation show good agreement.

\subsection{Estimating Residual Background from Distributions of Control Quantities}

There are limitations to the agreement achievable between simulated and experimental data with methods such as those shown in the previous section. In particular, the primary and secondary CR fluxes, which are important inputs for our simulation, are uncertainly known. Furthermore, efficiencies of the trigger and on-board filter might be underestimated or overestimated in the simulation. Therefore, we adjust the normalization of the total residual CR background independently in 15 energy bins between $100 \mathrm{MeV}$ and $\sim 600 \mathrm{GeV}$ to better describe the counts observed in the calibration data sample with a low $\gamma$-ray-to-background ratio defined in Section 3.6.4. The scaling is based on the events in the P7SOURCE class. For these events, the distributions of two event properties, the $P_{\text {all }}$ estimator (see Section 3.3.8) and the transverse size of the particle shower in the CAL, have different shapes for $\gamma$ rays and CRs. The shapes of the distributions are sufficiently distinct for extracting the contribution of both components by fitting a superposition of simulated $\gamma$ rays and CRs to the on-orbit data. Although the difference between the shapes of the distributions for $\gamma$ rays and CR background decreases with increasing energy for the $P_{\text {all }}$ estimator, it does increase for the transverse shower size. Therefore, the fit is performed on $P_{\text {all }}$ for energies $E \leqslant 3 \mathrm{GeV}$, and on the transverse shower size for $E>3 \mathrm{GeV}$. The CR background correction factors obtained by these fits are then used to adjust the residual background predicted by the simulations.

Figure 26 compares the distribution of the $P_{\text {all }}$ estimator and the transverse shower size between simulated and experimentally observed events after the normalizations of the predicted CR background and of the $\gamma$-ray simulation have been adjusted. Each plot refers to a representative energy band in which the fit was performed for the respective variable. Figure 27 shows the adjustment factors obtained in the fit as a function of energy. An adjustment factor of 1 corresponds to the CR background intensity predicted in the simulation. The adjustment factors vary between 0.7 and 1.6, depending on energy.

We use the predictions of the residual CR background from the MC simulation for the P7SOURCE, P7CLEAN, and P7ULTRACLEAN event classes multiplied by the adjustment factors in Figure 27 as our best estimate of the residual background. We use the largest adjustment factor (1.59), which is an indicator of the relative uncertainty of our determination of the residual background. This uncertainty is found to be $\sim 35 \%$, i.e., $(1.59-1.00) / 1.59=0.37$.

Figure 28 summarizes our best estimate of the differential particle background rates in the three high-purity event classes for the energy range between $100 \mathrm{MeV}$ and $600 \mathrm{GeV}$. Our background model is likely inaccurate below $100 \mathrm{MeV}$, and therefore the background contamination cannot be reliably determined by means of MC simulations in that energy range.

\subsection{Estimating Irreducible Backgrounds}

The term "irreducible" was introduced in Section 2.5.4 for CR-induced background with a well-reconstructed $\gamma$ ray inside 

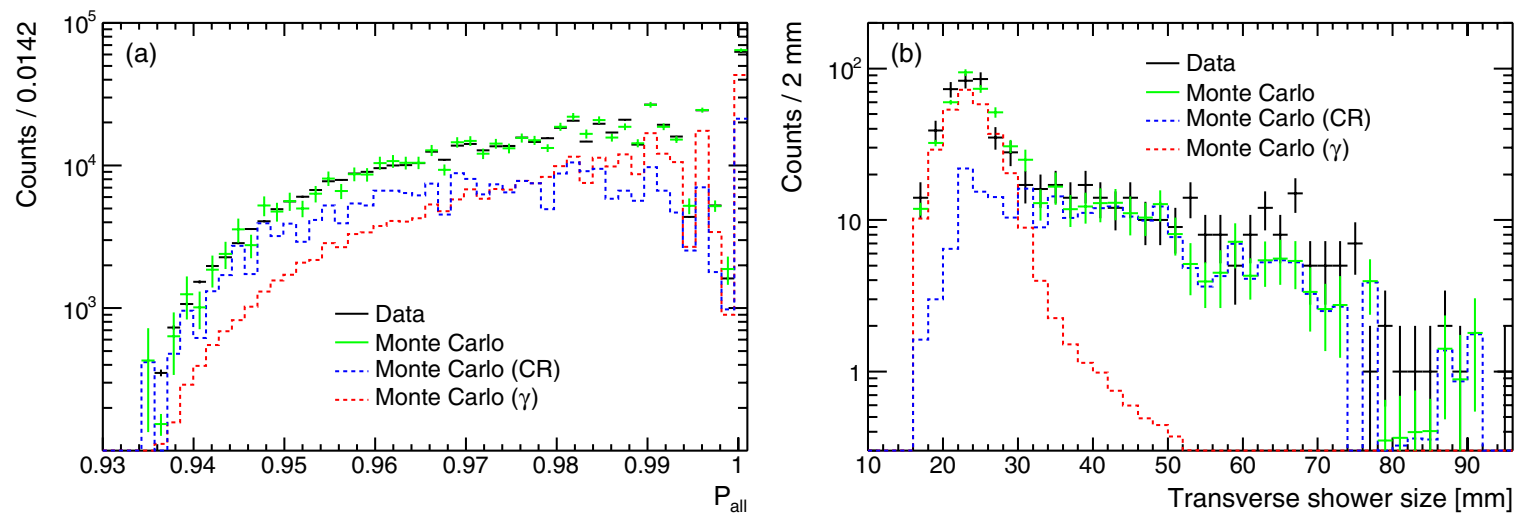

Figure 26. (a) Distribution of the $P_{\text {all }}$ estimator in the energy range $200-400 \mathrm{MeV}$ for $\gamma$ rays and residual CRs; (b) distribution of the transverse shower size in the energy range 32-64 GeV. The ranges are representative of the energy intervals in which the fit was performed for each of the variables. Only P7SOURCE events from high Galactic latitudes $|b|>50^{\circ}$ and longitudes $90^{\circ}<|l|<270^{\circ}$, which feature a low $\gamma$-ray-to-background ratio (i.e., the "dirty" calibration sample), are shown. The normalizations of the CR background simulation and the $\gamma$-ray simulation are adjusted to fit the data.

(A color version of this figure is available in the online journal.)

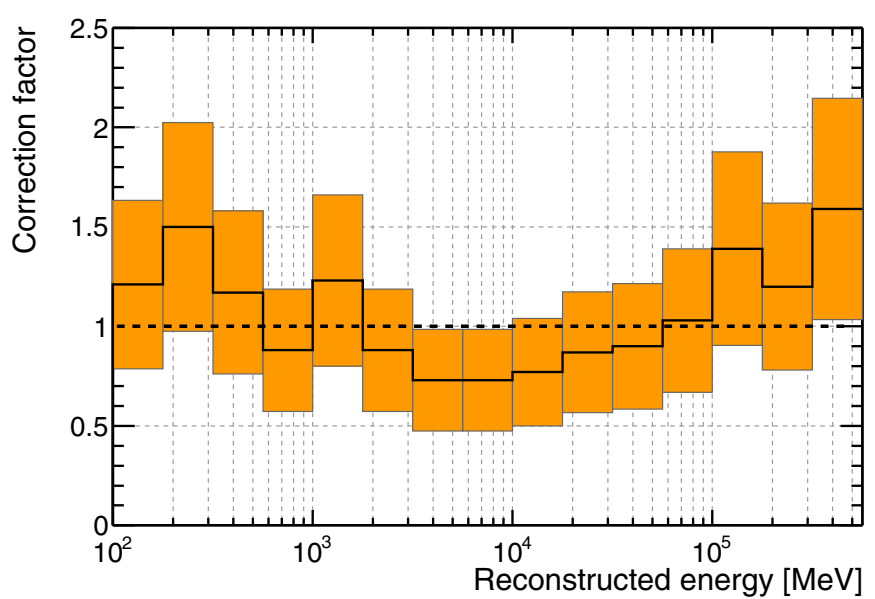

Figure 27. Correction factors for the CR background simulation as a function of energy, determined from a fit to the experimental data. The filled band shows the $35 \%$ systematic uncertainty. Note that the energies shown here are based on reconstruction under the hypothesis that the event is a $\gamma$ ray and that most high-energy protons deposit only a small fraction of their total energy in the LAT.

(A color version of this figure is available in the online journal.)

the LAT as its only signature and is therefore indistinguishable from $\gamma$ rays of cosmic origin. As the two main classes of irreducible backgrounds we listed the CR positrons that annihilate outside the detector, and the $\mathrm{CR}$ protons that inelastically scatter in the passive material surrounding the ACD. This irreducible background is mostly limited to energies below a few $\mathrm{GeV}$ due to its production mechanisms. We do not consider the $\gamma$-ray emission from the Earth's atmosphere as irreducible, as only $\gamma$ rays with large errors in reconstructed directions enter the samples usually chosen for high-level analysis. The contamination can be reduced by both a stricter selection on event quality to reject badly reconstructed $\gamma$ rays and by larger exclusion regions around the Earth.

An estimate of the fraction of irreducible background in the cleanest event class (i.e., P7ULTRACLEAN) is informative as it represents a lower limit to the achievable background rejection. Since the irreducible background cannot be separated from the data, we can deduce its amount only based on available MC information.

To determine the irreducible background from positron annihilations, one can compare the relative fractions of electrons and positrons surviving at different stages of the event selection. The secondary CR leptons between $100 \mathrm{MeV}$ and $3 \mathrm{GeV}$ passing the on-board filter are composed of $f_{\text {obf }}^{-} \approx 0.28$ of electrons and $f_{\mathrm{obf}}^{+} \approx 0.72$ of positrons. Technically, the positron component is the sum of a reducible and an irreducible part; however, at this stage, the data set is still overwhelmingly dominated by reducible charged CRs, so that the irreducible contribution is effectively negligible:

$$
f_{\mathrm{obf}}^{+}=f_{\mathrm{obf}, \mathrm{red}}^{+}+f_{\mathrm{obf}, \text { irr }}^{+} \approx f_{\mathrm{obf}, \mathrm{red}}^{+} .
$$

The secondary CR leptons passing the P7ULTRACLEAN selection are composed of $f_{\mathrm{uc}}^{-} \approx 0.10$ of electrons and $f_{\mathrm{uc}}^{+}=f_{\mathrm{uc}, \text { red }}^{+}+$ $f_{\text {uc,irr }}^{+} \approx 0.90$ of positrons. Since the reducible electron and positron components are indistinguishable in the LAT, they scale identically

$$
f_{\mathrm{uc}, \text { red }}^{+}=\frac{f_{\mathrm{obf}, \mathrm{red}}^{+} f_{\mathrm{uc}}^{-}}{f_{\mathrm{obf}}^{-}} \approx \frac{f_{\mathrm{obf}}^{+} f_{\mathrm{uc}}^{-}}{f_{\mathrm{obf}}^{-}} ;
$$

therefore, we have

$$
f_{\mathrm{uc}, \mathrm{irr}}^{+}=1-f_{\mathrm{uc}}^{-}-f_{\mathrm{uc}, \mathrm{red}}^{+} \approx \frac{f_{\mathrm{obf}}^{-}-f_{\mathrm{uc}}^{-}}{f_{\mathrm{obf}}^{-}} \approx 0.64 .
$$

(i.e., $\approx 64 \%$ of the secondary leptons in the P7ULTRACLEAN event class are irreducible background events from positron annihilations).

The amount of irreducible background below $1 \mathrm{GeV}$ from inelastic scatters of protons can be estimated by evaluating the fraction of the residual simulated CR protons that does not enter the volume surrounded by the ACD. This is the case for about 95\% of the simulated CR protons passing the P7ULTRACLEAN selection.

For geometric reasons these scatters predominantly occur at the edges of the LAT (about $75 \%$ of the residual CR protons, while the remaining $25 \%$ scatter in the spacecraft body). Figure 29 shows the positions of the projected intersections with the top ACD plane for simulated CR proton events surviving the P7ULTRACLEAN selection with reconstructed energies below $1 \mathrm{GeV}$. An enhancement of the tracks from the edges of the LAT is clearly visible, but smeared out due to the finite accuracy of the direction reconstruction. This feature, in fact, suggests the possibility to suppress this type of irreducible background by 

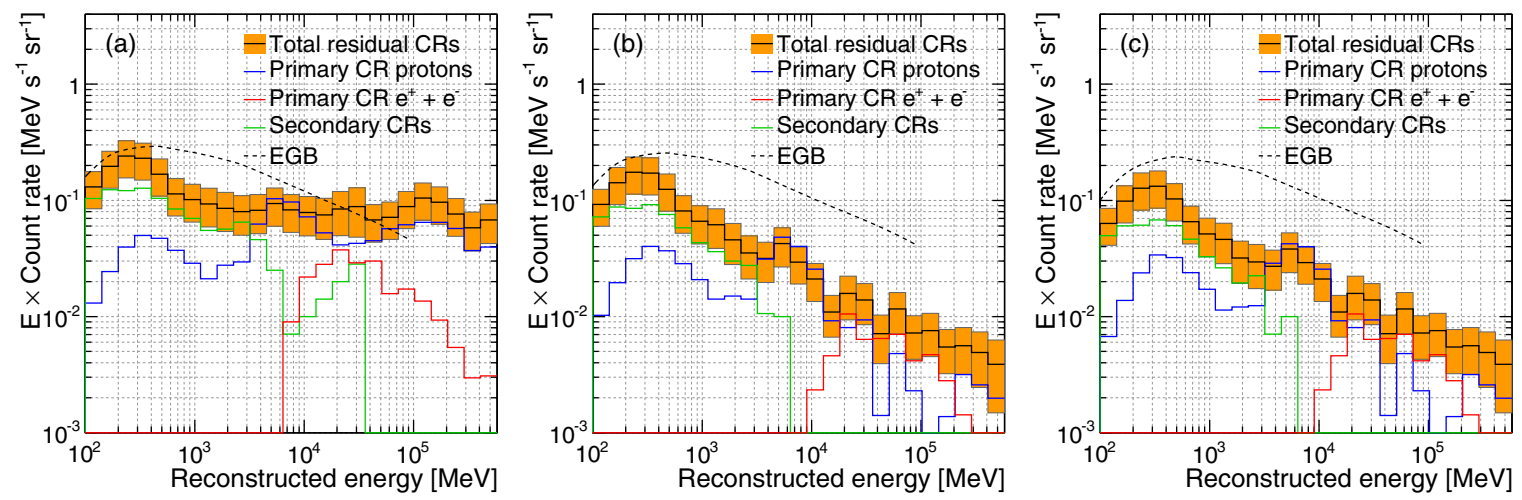

Figure 28. Best estimates of differential rates of residual particle backgrounds for the P7SOURCE (a), P7CLEAN (b), and P7ULTRACLEAN (c) event classes. Individual contributions from primary CR protons, primary CR electrons, and the secondaries from CR interactions are shown; the corresponding count rates for the extragalactic $\gamma$-ray background measured by Fermi (Abdo et al. 2010e) are also overlaid for comparison.

(A color version of this figure is available in the online journal.)

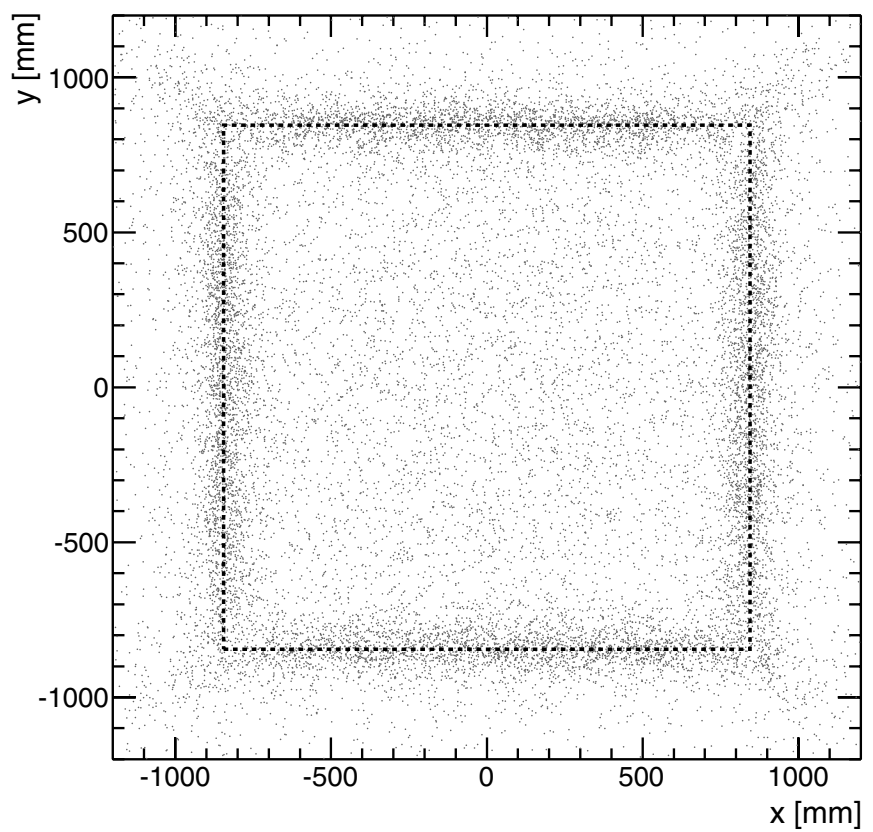

Figure 29. Positions of the projected intersections with the top ACD plane for simulated CR proton events surviving the P7ULTRACLEAN selection. The dashed line marks the edges of the area covered by the ACD.

rejecting events from the edges of the LAT. However, due to the size of the PSF at low energies tracks intersecting large regions of the LAT surface would have to be vetoed, resulting in an unacceptable loss of effective area. Therefore, such a veto has not been implemented in any of the event classes.

\subsection{Treatment of Particle Backgrounds}

After the event selection, even the purest class will contain residual background from misclassified CRs. This contribution needs to be accounted for in the modeling used for spectral and spatial analysis of celestial $\gamma$-ray sources. In particular, in the maximum likelihood model fitting framework of ScienceTools, the misclassified CRs must either be modeled separately or subsumed into one of the diffuse $\gamma$-ray components.

The simplest approach is to add a new source to the model to account for the residual background due to particle leakage. In most cases, since $\mathrm{CR}$ rates are related to geomagnetic (i.e., Earth) coordinates, for time intervals greater than a few months, residual background events become approximately isotropically distributed in sky coordinates.

The possibility of deriving an effective background template rests mainly on the assumption that the incidence angle dependence of $A_{\text {eff }}$ is the same for CRs and $\gamma$ rays. In many cases, we also use a simplifying assumption that the CR contamination rates in front- and back-converting events scale with the relative acceptances. However, as we will discuss below and in Section 4.6, that is not always the case.

The heuristic nature of such a source is evident if one considers how it changes for different event selections. When we analyze a real $\gamma$-ray source using different event class selections we expect to find the same spectral distribution within the known systematics; on the other hand, this template depends on the amount of residual background and therefore on the $\mathrm{CR}$ rejection efficiency of the event class, so the templates we derive for the various event selections are dramatically different. Each event class requires a dedicated background template.

Under the above assumptions, any isotropic $\gamma$-ray component (e.g., the contribution of unresolved extragalactic sources) is not separable from the background leakage by means of a standard high-level source analysis (likelihood analysis) without additional knowledge (e.g., of spectral differences), so the two components are collected into a single term, simply called the isotropic template. ${ }^{78}$ To derive an isotropic template for a given event selection we perform a likelihood analysis of the highlatitude $(|b|>30)$ sky, including all resolved individual sources and a model of the Galactic interstellar emission, ${ }^{79}$ fitting the spectrum of the isotropic component. It follows that the derived isotropic template depends on the assumed model for the Galactic interstellar emission, notably on the inverse Compton component, which is smooth and far from negligible even at high Galactic latitudes, since the interstellar radiation field and CR electrons are broadly distributed about the Galactic plane. Therefore, each Galactic interstellar emission model requires a different isotropic template.

Between $\sim 400 \mathrm{MeV}$ and $10 \mathrm{GeV}$ the assumptions mentioned above are rather good. In the 2FGL catalog analysis (Nolan et al. 2012), a single isotropic template was used and no significant systematics were observed above $400 \mathrm{MeV}$. Outside this energy range, the rate of residual background events in the back section

\footnotetext{
78 For example, iso_p7v6source.txt, available at http://fermi.gsfc.nasa.gov/ssc/data/access/lat/BackgroundModels.html.

79 For example, gal_2yearp7v6_v0.fits, available at

http://fermi.gsfc.nasa.gov/ssc/data/access/lat/BackgroundModels.html.
} 
is appreciably greater than for the front section: the use of a single isotropic template (describing a weighted average of front and back background contamination) leads to a small hardening of measured spectra of point sources (Nolan et al. 2012). The effect is maximum for low-significance, soft sources: on average the spectral indices of power-law spectra are hardened by less than half of the typical uncertainty in the measured spectral index. It is preferable to derive separate isotropic templates for front and back and use them in a combined likelihood approach, ${ }^{80}$ if front and back events are kept separate in the analysis, but the magnitude of this effect does not warrant such a complication for many analyses.

To derive the true isotropic $\gamma$-ray component from the measured isotropic component it is necessary to separately estimate and subtract the amount of residual background contamination (see Section 4.3).

The strategy to account for the CR background by means of an isotropic template, however, fails in the case of the $\gamma$-ray emission from the Earth limb. Residual background events in the FoV due to limb emission reconstructed in the tails of the PSF will produce a distinct pattern on the sky; its shape will depend on the pointing history and the time and energy ranges under consideration, and will be different for front- and backconverting events.

A more stringent cut on $\theta_{z}$ will reduce the contamination at the expense of exposure in certain regions of the sky. In particular, below $100 \mathrm{MeV}$ exclusion regions to effectively eliminate the residual background become prohibitively large and significant Earth limb emission remains in the data sample for the commonly used zenith angle limit of $100^{\circ}$.

For the analysis leading to the 2FGL catalog, $\gamma$ rays with $\theta_{z}>100^{\circ}$ were rejected. The remaining Earth limb emission was characterized by a template derived from the residual emission visible in the 50-68 MeV energy band, which extended up to about $400 \mathrm{MeV}^{81}$ (for details see Nolan et al. 2012). However, this template should not be used for periods much shorter than two years.

Finally, residual background associated with mischaracterized "back-entering" $\gamma$ rays (see Section 2.5.3), is another specific background that does not follow the $\gamma$-ray acceptance. The probability of accepting "back-entering" $\gamma$ rays into the P7SOURCE event selection is $\sim 1000$ times smaller than for "front-entering" $\gamma$ rays and they are assigned directions roughly $180^{\circ}$ away from the true directions. We consider the effect of this background in Section 4.6 and find that treating it as part of the isotropic background does not introduce significant errors into analyses of point sources.

\subsection{Propagating Systematic Uncertainties to High-level Science Analysis}

As discussed in the previous section, residual CR background is treated as an isotropic fictitious $\gamma$-ray source in highlevel science analysis of astrophysical $\gamma$-ray sources. As this approximation becomes less than perfect, significant systematic uncertainties can arise.

In addition, a slight inconsistency between front and back $A_{\text {eff }}$ (see Section 5.5) complicates the issue further, causing additional uncertainties when separately deriving the isotropic

\footnotetext{
${ }^{80}$ For example, isotropic_iem_front_v02.txt and isotropic_iem_back_v02.txt, available at http://fermi.gsfc.nasa.gov/ssc/data/access/lat/ BackgroundModels.html.

81 Available at http://fermi.gsfc.nasa.gov/ssc/data/access/lat/2yr_catalog.
}

emission for the two selections. In general, we can quantify the resulting systematic uncertainties by comparing estimates obtained from the front-only selection with the full data set.

The isotropic templates derived for the 2FGL catalog analysis for P7SOURCE_V6 events (and released via the FSSC) can be used for analyses spanning timescales of many months. On short timescales, especially less than the $\sim 53.4$ day precession period of the Fermi orbit, changes in the distribution of geomagnetic latitudes through which the LAT passes cause the residual background rates to be strongly dependent on the exact orbital history of the spacecraft and on the CR spectra at different geomagnetic locations. Analyses based on short time selections could do better either by using a dedicated estimate of the $\mathrm{CR}$ and isotropic backgrounds, e.g., by using a nearby control region, or one could take particular care to assess the impact of a possibly incorrect spectrum and spatial distribution of the background counts. Often this is done by allowing the isotropic component some freedom in the fitting procedure; see, for example, Section 8.4.

As discussed in Sections 4.1, 4.3, and 4.4, P7CLEAN and P7ULTRACLEAN event classes have much lower levels of background contamination than P7SOURCE. Accordingly, these samples can be used to study the dependence of any particular analysis on the level of particle background contamination in P7SOURCE analysis.

Finally, we have studied the distribution of residual CR backgrounds in the P7SOURCE event sample $b_{s}(E, \hat{p})$ by comparing the observed counts distribution $n_{s}(E, \hat{p})$ in that sample with the predicted distribution $\tilde{n}_{s}(E, \hat{p})$, which we obtain by scaling the distribution of the P7ULTRACLEAN $n_{u}(E, \hat{p})$ sample by the ratio of the exposure calculated with the P7SOURCE_V6 IRFs $\mathcal{E}_{s}(E, \hat{p})$ to the exposure calculated with the P7ULTRACLEAN_V6 IRFs $\mathcal{E}_{u}(E, \hat{p})$. Specifically,

$$
\begin{aligned}
& \tilde{n}_{s}(E, \hat{p})=n_{u}(E, \hat{p}) \frac{\mathcal{E}_{s}(E, \hat{p})}{\mathcal{E}_{u}(E, \hat{p})}, \\
& b_{s}(E, \hat{p})=n_{s}(E, \hat{p})-\tilde{n}_{s}(E, \hat{p}) .
\end{aligned}
$$

We studied the correlation between residual background and exposure as a function of energy. The detailed results are beyond the scope of this paper, but in general $b_{s}$ is not strictly proportional to $\mathcal{E}_{s}$. This implies that the effective acceptance for residual CR backgrounds in the P7SOURCE event sample is not the same as for $\gamma$ rays.

Although the spatial distribution of the residual CR background could impact studies of large-scale diffuse emission, the variation across a typical $\sim 20^{\circ}$ ROI used when analyzing point sources is less that the variation in the exposure $(2 \%-5 \%$, depending on the energy). Furthermore, for bright sources with sufficient statistics to make high-precision measurements, the correlation factor between the source parameters and the normalization of the isotropic component typically has a very small magnitude $(<0.03)$. Accordingly, we neglect the spatial variations in the $\mathrm{CR}$ background contamination when performing point-source analyses.

\section{EFFECTIVE AREA}

In order to correctly evaluate the spectra of astrophysical $\gamma$-ray sources, we need to know the effective collecting area of the LAT. In fact, $A_{\text {eff }}$ depends on the geometrical cross section of the LAT as well as the efficiency for converting and correctly identifying incident $\gamma$ rays. Because of the complexity of determining these, we use high statistics MC simulations 

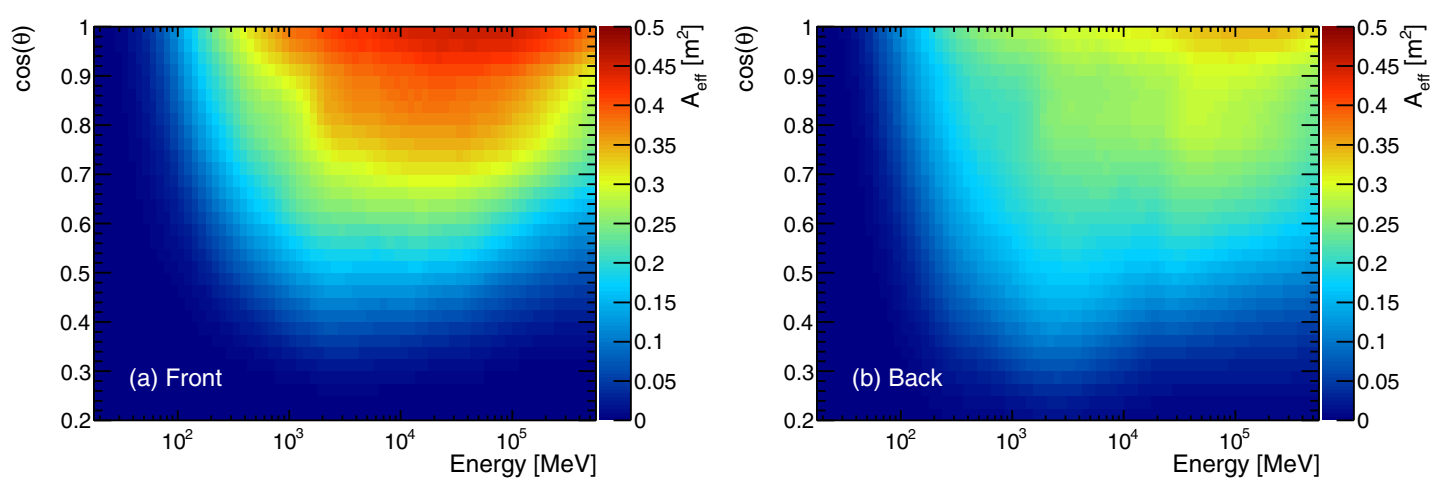

Figure 30. Graphical representation of the effective area tables for the P7SOURCE_V6 class, front (a) and back (b) sections of the LAT.

(A color version of this figure is available in the online journal.)

to evaluate $A_{\text {eff }}$. We then quantify any discrepancies between simulations and flight data, and if needed, correct the MC-based $A_{\text {eff }}$ accordingly.

As mentioned in Section 1, we express the effective area as a function of the incident $\gamma$-ray energy and direction in the LAT instrument frame. Therefore, the exposure $(\mathcal{E})$ at a given energy for any point in the sky depends on the effective area and the observing profile (see Equation (3)).

In practice, the observing profile depends on the direction in the sky, and is accurately known. Therefore, the uncertainties on $A_{\text {eff }}(E, \theta, \phi)$ are the dominant source of instrument-related systematic error. Of course, we must also consider the uncertainties on our measurements of the $\gamma$-ray direction (i.e., the PSF) and the $\gamma$-ray energy (i.e., the energy dispersion). However, as we will show in the next three sections, in many cases the uncertainty of $A_{\text {eff }}$ is more important to the analyses than those of the PSF and the energy dispersion.

In Sections 5.1 and 5.2 we will describe how we generate tables of $A_{\text {eff }}$ as a function of energy and incidence angle (for the front and back sections of the LAT separately), and how we apply small corrections to those tables to account for variations of $A_{\text {eff }}$ with the orbital position and azimuthal direction of the incoming $\gamma$ ray. Then in Section 5.3 we will describe how we have validated the MC predictions of the $\gamma$-ray selection efficiency for all the stages of the analysis using calibration samples within the flight data set, while in Section 5.4 we will describe corrections to the $A_{\text {eff }}$ tables motivated by disagreement between the measured and predicted efficiency in one step of the selection process. Finally, in Sections 5.5-5.7 we will evaluate the systematic uncertainties on $A_{\text {eff }}$ and show how we propagate these uncertainties into estimated systematic errors on measured astrophysical quantities such as fluxes and spectral indices.

\subsection{Effective Area Studies with Monte Carlo Simulations}

The starting point of the $A_{\text {eff }}$ evaluation is a dedicated allGamma sample (Section 2.5.3). Since the $\gamma$ rays are generated uniformly in $\log (E)$ and solid angle, the effective area in any of the bins, in which the parameter space is partitioned, can be expressed in terms of the total number of generated events $N_{\text {gen }}$ and the number of events $n_{i, j, k}$ passing the $\gamma$-ray selection criteria within the specific bin centered at $E=E_{i}, \theta=\theta_{j}$, and $\phi=\phi_{k}$ :

$$
\begin{aligned}
A_{\mathrm{eff}}\left(E_{i}, \theta_{j}, \phi_{k}\right)= & \left(6 \mathrm{~m}^{2}\right)\left(\frac{n_{i, j, k}}{N_{\mathrm{gen}}}\right)\left(\frac{2 \pi}{\Delta \Omega_{j, k}}\right) \\
& \times\left(\frac{\log _{10} E_{\mathrm{max}}-\log _{10} E_{\mathrm{min}}}{\log _{10} E_{\mathrm{max}, i}-\log _{10} E_{\mathrm{min}, i}}\right),
\end{aligned}
$$

where $\Delta \Omega_{j, k}$ is the solid angle subtended by the bin $j, k$ in $\theta$ and $\phi, E_{\min }$ and $E_{\max }$ give the energy range of the allGamma sample, and $E_{\min , i}$ and $E_{\max , i}$ are the boundaries of the $i$ th energy bin. (See Section 2.5.3 for more details about the numerical factors and about the allGamma simulations in general.) In practice, since the effective area is routinely averaged over $\phi$ in scientific analysis, we factor out the $\phi$ dependence and rewrite Equation (10) as

$$
\begin{aligned}
A_{\mathrm{eff}}\left(E_{i}, \theta_{j}, \phi_{k}\right)= & \left(6 \mathrm{~m}^{2}\right)\left(\frac{n_{i, j}}{N_{\mathrm{gen}}}\right)\left(\frac{2 \pi}{\Delta \Omega_{j}}\right) \\
& \times\left(\frac{\log _{10} E_{\mathrm{max}}-\log _{10} E_{\mathrm{min}}}{\log _{10} E_{\mathrm{max}, i}-\log _{10} E_{\mathrm{min}, i}}\right) \\
& \times R\left(E_{i}, \theta_{j}, \phi_{k}\right),
\end{aligned}
$$

where $R(E, \theta, \phi)$ is a small (of the order of $10 \%$ ) correction factor whose average over $\phi$ is 1 by construction for any $E$ and $\theta$ (see Section 5.2.3 for more details). Examples of effective area tables averaged over $\phi$ are shown in Figure 30.

When describing the instrument performance, we more commonly show the effective area at normal incidence as a function of the energy or the angular dependence of the effective area for a given energy (usually $10 \mathrm{GeV}$ ), as shown in Figure 31.

The integral of the effective area over the solid angle, called the acceptance

$$
\begin{aligned}
\mathcal{A}(E) & =\int A_{\mathrm{eff}}(E, \theta, \phi) d \Omega \\
& =\int_{0}^{\frac{\pi}{2}} \int_{0}^{2 \pi} A_{\mathrm{eff}}(E, \theta, \phi) \sin \theta d \theta d \phi,
\end{aligned}
$$

is another widely used performance measure and is shown as a function of energy in Figure 32.

Formally, the FoV is defined, at any given energy, as the ratio between the acceptance and the on-axis effective area:

$$
\operatorname{FoV}(E)=\frac{\mathcal{A}(E)}{A_{\text {eff }}(E, \theta=0)} .
$$

Figure 33 shows that the peak FoV of the LAT for the P7SOURCE event class is of the order of $2.7 \mathrm{sr}$ between 1 and $10 \mathrm{GeV}$. At lower energies the FoV decreases with energy, as $\gamma$ rays converting in the TKR at large angles pass through comparatively more material, and therefore are less likely to trigger the instrument. A similar (smaller) effect is observed at very high energy, where, due to backsplash from the CAL, it becomes more difficult to reconstruct events at large angles. 

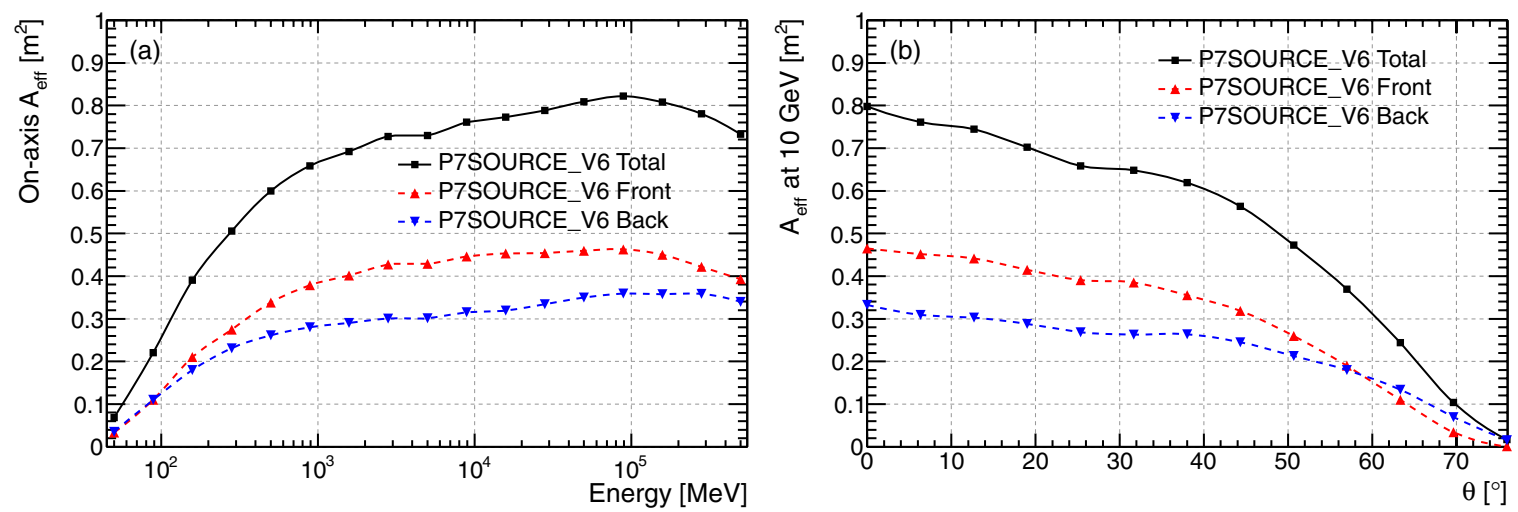

Figure 31. On-axis effective area as a function of the energy (a) and angular dependence (b) of the effective area at $10 \mathrm{GeV}$ for the P7SOURCE class. (A color version of this figure is available in the online journal.)
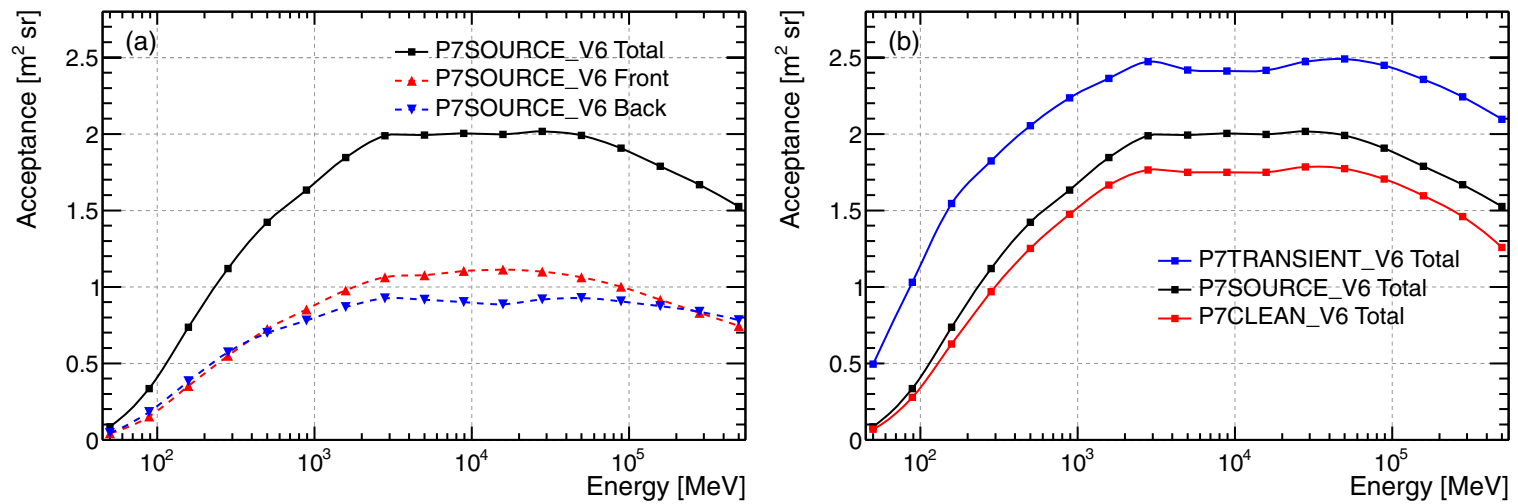

Figure 32. Acceptance as a function of energy for the P7SOURCE class (a) and for the other standard $\gamma$-ray classes (b).

(A color version of this figure is available in the online journal.)

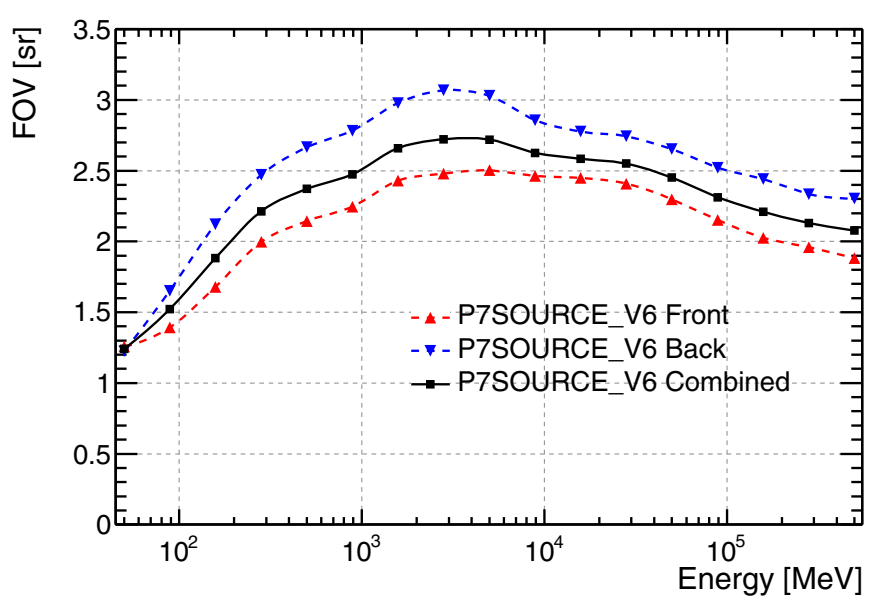

Figure 33. LAT FoV as a function of the energy for the P7SOURCE class. Frontand back-converting events are shown separately.

(A color version of this figure is available in the online journal.)

Finally, we note that for geometrical reasons (we require events in the standard classes to intersect the CAL) the FoV for the back section is typically larger than that for the front section.

\subsection{Corrections to the Effective Area Derived from Simulations}

In this section, we describe three refinements we made to the effective area characterization based on experience with flight data. In all three cases we have simulated the effects that we had previously ignored or averaged out, but that we discovered could significantly impact particular scientific analyses.

\subsubsection{Correction for Ghost Events}

As explained in more detail in Section 2.1.4, after the start of LAT operations, it became apparent that ghost signals led to a significant decrease in effective area with respect to the prelaunch estimates, for which this effect was not considered. The overlay procedure used to account for this effect, first introduced in the P6_V3 set of IRFs, is described in detail in Section 2.5.1 and its impact on the effective area is shown in Figure 34.

\subsubsection{Live Time Dependence}

The effect of ghost signals is corrected on average as described in the previous section. A smaller correction is necessary to account for the detailed dependence of $A_{\text {eff }}$ on the $\mathrm{CR}$ rates. To account for this we need an estimator for the rate of CRs entering the LAT; the obvious one is the trigger rate, but technical issues make this choice impractical. A variable that can be easily obtained from the pointing history files and which is linearly correlated with trigger rate is the live time fraction $F_{l}$, the fraction of the total observing time in which the LAT is triggerable and not busy reading out a previous event. The average value of $F_{l}$ is $\sim 90 \%$ and varies between $82 \%$ and 92\% over the Fermi orbit.

We bin events from a sample of periodic triggers according to the corresponding live time fraction and for each bin we produce 


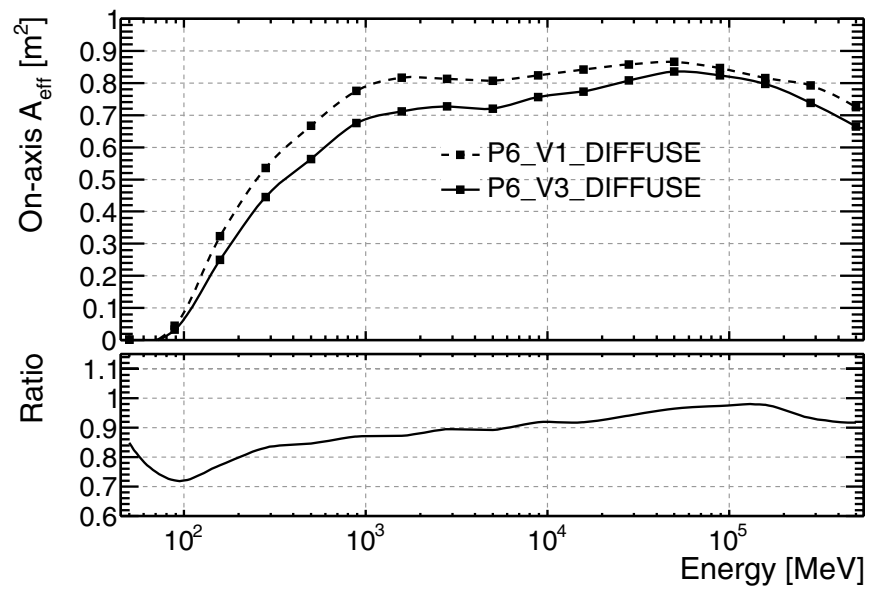

Figure 34. Effective area at normal incidence for the P6_DIFFUSE class, in the pre-launch version (P6_V1_DIFFUSE, not corrected for ghost effects) and in the updated version including a modeling of ghost effects (P6_V3_DIFFUSE). Adapted from Rando \& The Fermi LAT Collaboration (2009).

a dedicated allGamma simulation (Section 2.5.3); for each of the resulting overlay data sets we derive $A_{\text {eff }}$. We have found the dependence on the incidence angle to be small and so we choose to neglect it when studying this effect. Furthermore, we have found that at a given energy $A_{\text {eff }}$ varies linearly with the live time fraction. We perform a linear interpolation in each energy bin in accord with

$$
A_{\mathrm{eff}}\left(E, F_{l}\right)=A_{\mathrm{eff}}(E) \cdot\left(c_{0}(E) F_{l}+c_{1}(E)\right)
$$

separately for front and back events.

In Figure 35, we plot $c_{0}$ and $c_{1}$ as a function of energy for front-converting events in the P7SOURCE event class. As shown by the solid lines, we use a simple piecewise linear fit to describe the energy dependence and the fit parameters are stored in the $A_{\text {eff }}$ tables. As we mentioned, the effective area derived from allGamma simulations that have overlaid periodic trigger events is effectively corrected for the average effect of this live time dependence, so we treat this additional correction (which can be positive or negative) as a modulation of the tabulated $A_{\text {eff }}$. Correction parameters are read in from the $A_{\text {eff }}$ files of each set of IRFs, and used to correct the calculated exposures.

The resulting corrections to the average $A_{\text {eff }}$ can reach $-30 \%$ at $100 \mathrm{MeV}$, decreasing at higher energies to $<5 \%$ above $\sim 10 \mathrm{GeV}$. The uncertainties in the corrections are much smaller; studies using flight data confirm the MC-based predictions to better than $2 \%$. Note that over a 53.4 day orbital precession

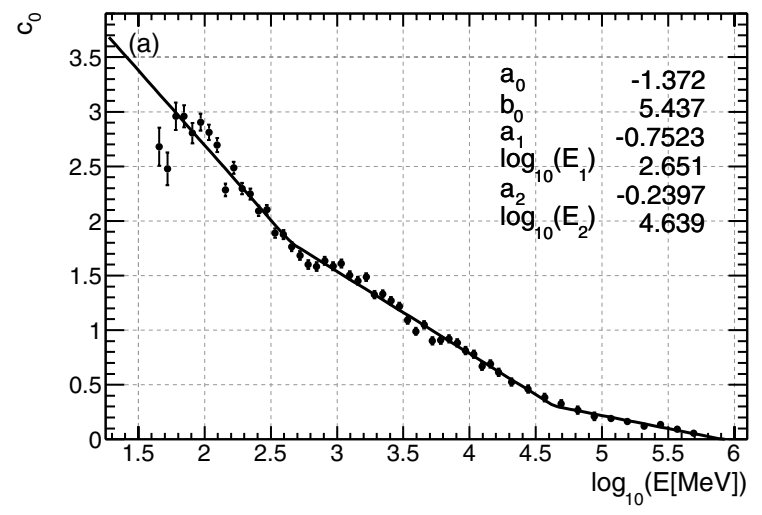

period this effect will tend to the overall average correction described in Section 5.2.1, with less than $1 \%$ variation across the sky.

Since the correction to the effective area is based on live time fraction, which is very strongly correlated with the CR intensity, it avoids any direct biases from long term changes in the CR intensity associated with the influence of solar activity on the geomagnetic field. However, the correction does not address the possibility that the $\mathrm{CR}$ population changes during the solar cycle in such a way as to change the effective area dependence on the live time fraction. Given the small change in the daily averaged LAT trigger accept rate observed in the mission to date $(<5 \%)$, we neglect this effect.

\subsection{3. $\phi$ Dependence}

The tabulated values of $A_{\text {eff }}$ are averaged over the azimuthal angle of incidence and shown in Figure 36. Much of the azimuthal dependence of the effective area is geometrical, due to the square shape of the LAT and the alignment of the gaps along the $x$ - and $y$-axes. The rms variation of the effective area as a function of $\phi$ is typically of the order of $5 \%$ and exceeds $10 \%$ only at low energies $(<100 \mathrm{MeV})$ or far off-axis $\left(\theta>60^{\circ}\right)$ where the effective area is small, and at very high energies $(>100 \mathrm{GeV})$ where the event rate is small.

In order to parameterize the azimuthal dependence of $A_{\text {eff }}$, we fold the azimuthal angle into the $\left[0^{\circ}, 45^{\circ}\right]$ interval and remap it into $[0,1]$ using the following transformation:

$$
\xi=\frac{4}{\pi}\left|\left(\phi \bmod \frac{\pi}{2}\right)-\frac{\pi}{4}\right|
$$

(the transformation maps $0^{\circ}$ to $1,45^{\circ}$ to $0,90^{\circ}$ to 1 , and so on). The allGamma events are binned in energy and $\theta$ and in each bin a histogram of $\xi$ is fitted (see Figure 37 for an example). Front- and back-converting events are treated separately. The fitting function is

$$
f(\xi)=1+q_{0} \xi^{q_{1}}
$$

The absolute scale is not important: we normalize the correction to result in an average multiplicative factor of 1 , so that the average $A_{\text {eff }}$ is tabulated. The fitted parameters $q_{0}$ and $q_{1}$ are stored in the $A_{\text {eff }}$ tables.

We note in passing that the default for high-level analyses is to disregard the azimuthal variations when calculating exposures because they average out when intervals of months or longer are

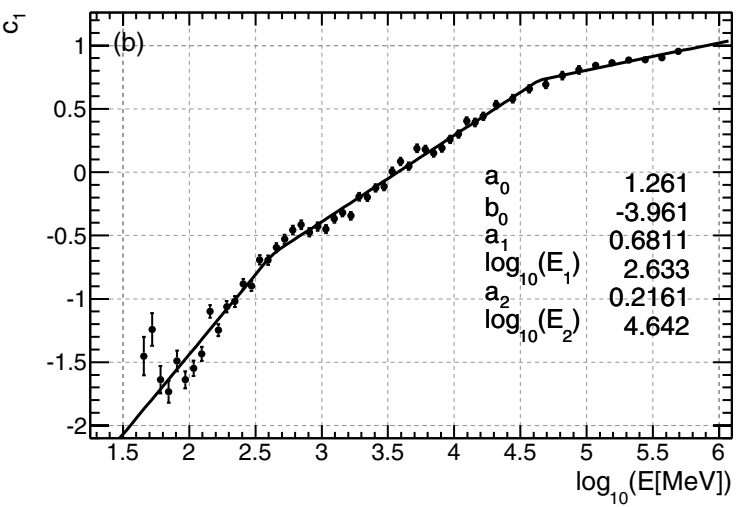

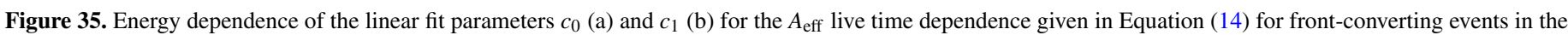
P7SOURCE : : FRONT event class. 


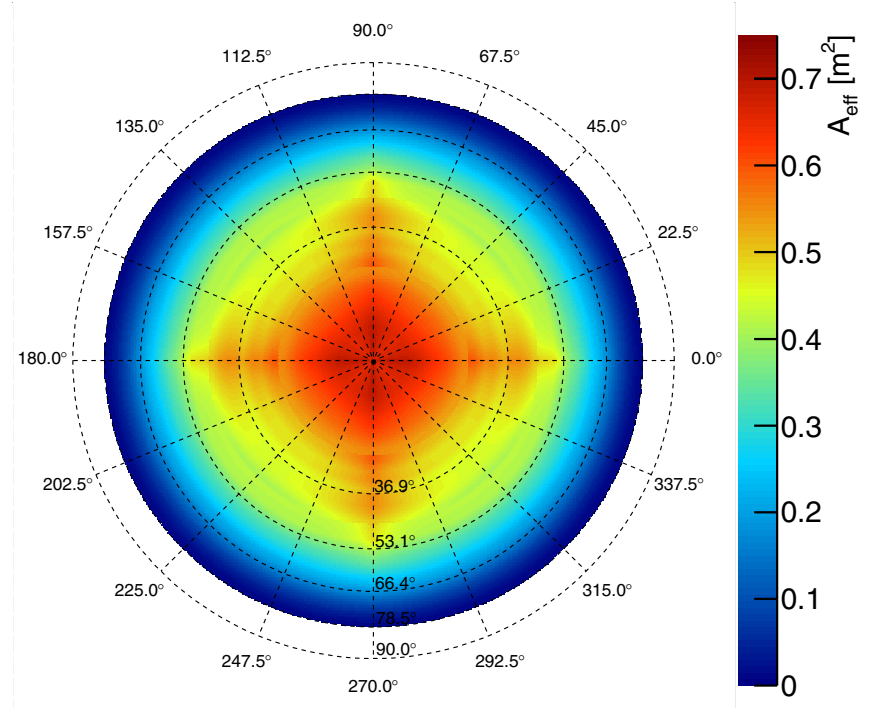

Figure 36. Total effective area at $10 \mathrm{GeV}$ as a function of the incidence angle $\theta$ and the azimuthal angle $\phi$ for the P7SOURCE event class. The plot is shown in a zenith equal-area projection with the LAT boresight at the center of the image; the concentric rings correspond to 0.2 increments in $\cos (\theta)$.

(A color version of this figure is available in the online journal.)

considered. ${ }^{82}$ Although the combined $\theta$ and $\phi$ dependence of the observing profile averages out only on year-long timescales, the eightfold symmetry of the LAT combined with the rotation of the $x$-axis to track the Sun results in effective averaging over $\phi$ on short timescales. In fact, we have found that ignoring the $\phi$ dependence of the effective area results in only a small variation of the exposure on $12 \mathrm{hr}$ timescales ( $<3 \% \mathrm{rms}$ at all energies).

\subsection{Step-by-step Performance of Cuts and Filters}

Before describing the studies we performed to validate our event selections, it is worth recalling that we require background rejection of $\sim 10^{6}$ while retaining high efficiency for $\gamma$ rays. To achieve this we must select events based on many different criteria that are applied in several stages (see Sections 2 and 3), which complicates the task of measuring the overall efficiency. For validation purposes, we specifically examine the agreement of the selection efficiencies $(\eta)$ between flight data and MC simulation. Therefore, the most relevant quantity is

$$
R=\frac{\eta_{\text {data }}}{\eta_{\mathrm{mc}}}
$$

which is in general a function of direction, energy, and conversion point of the incident $\gamma$ ray. The approach described in this section can be used both to quantify the systematic uncertainties in $A_{\text {eff }}$ and possibly to correct the IRFs derived from the MC simulations, in cases of severe discrepancies with the flight data (e.g., see Section 5.4).

\subsubsection{Background Subtraction}

To measure the efficiency of a cut on flight data, we can perform background subtraction before and after the cut and compare the excess in the signal region in the two cases. To

\footnotetext{
82 Information on how to include the $\phi$ dependence of the effective area in exposure calculations can be found at http://fermi.gsfc.nasa.gov/ssc/data/ analysis/scitools/binned_likelihood_tutorial.html and http://fermi.gsfc.nasa.gov/ssc/data/analysis/scitools/help/gtltcube.txt.
}

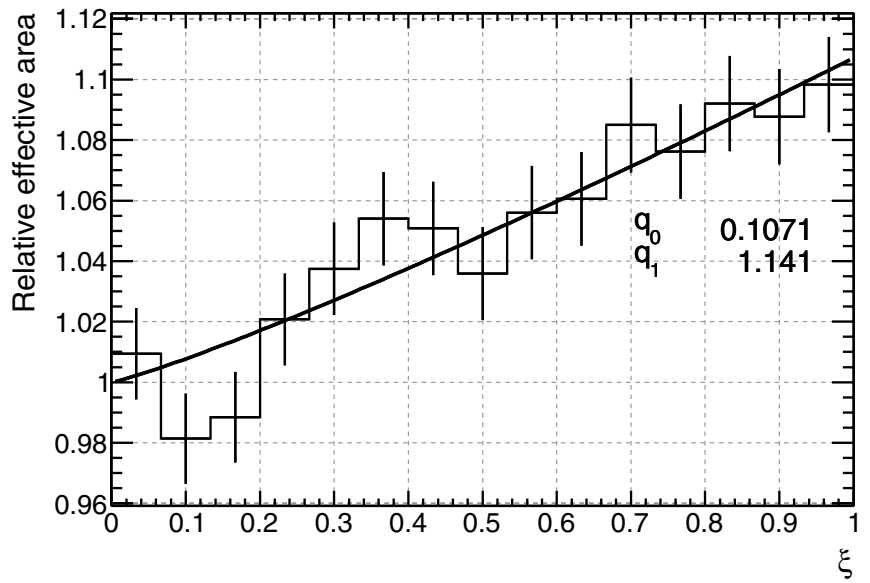

Figure 37. Example of $A_{\text {eff }}$ azimuthal dependence fit. The plot refers to the bin centered at $7.5 \mathrm{GeV}$ and $30^{\circ}$ for the P7SOURCE class, front section-a similar fit is performed in each $(E, \theta)$ bin. The folded azimuthal angle $\xi$ is defined in Equation (15) and the fit function in Equation (16). Note that this plot shows $A_{\text {eff }}$ relative to $A_{\text {eff }}$ for $\xi=0$.

the extent that the background subtraction is correct, the cut efficiency is simply the ratio of the number of backgroundsubtracted events before and after the cut we are testing

$$
\eta=\frac{n_{s, 1}-r n_{b, 1}}{n_{s, 0}-r n_{b, 0}}
$$

where the subscripts $s$ and $b$ indicate the signal and background regions, the subscripts 0 and 1 indicate the samples before and after the cut under test, and $r$ is the ratio of the size of the background region to the size of the signal region. Note that the sample after the cut is a subset of the sample before the cut. Therefore, for a reasonable sample size $\left(n_{s}>10\right)$, the statistical uncertainty of the efficiency is

$$
\delta \eta=\left[\frac{\left[\left(n_{s, 0}-n_{s, 1}\right)+r^{2}\left(n_{b, 0}-n_{b, 1}\right)\right] \eta^{2}+\left(n_{s, 1}+r^{2} n_{b, 1}\right)(1-\eta)^{2}}{\left(n_{s, 0}-r n_{b, 0}\right)^{2}}\right]^{1 / 2} .
$$

An example application of this technique using data from the Vela calibration data set is shown in Figure 38.

In many cases our cuts have significant overlap-two cuts may reject many of the same events. In such cases, measuring the efficiency of the second cut after the first cut has been applied would give a quite different result than measuring the efficiency of the second cut without the first cut. Therefore, whenever possible, we use the background subtraction technique described above to evaluate the efficiency of each step of our event selection both independently of the other steps as well as after all the other cuts have been applied.

\subsubsection{Track-finding and Fiducial Cut Efficiency}

Although the track-finding and fiducial cuts are applied midway through our event selection process, we choose to discuss them first for two reasons: (1) we require an event direction to be able to perform a background subtraction on flight data and (2) we express $A_{\text {eff }}$ as a function of $E$ and $\theta$. Therefore, for performance studies we need to apply some minimum event quality and fiducial cuts before considering events for analysis. The standard cuts require at least one track found, with at least $5 \mathrm{MeV}$ of energy in the CAL and that the track extrapolates through at least four radiation lengths in the CAL (see Section 3.4.1). 

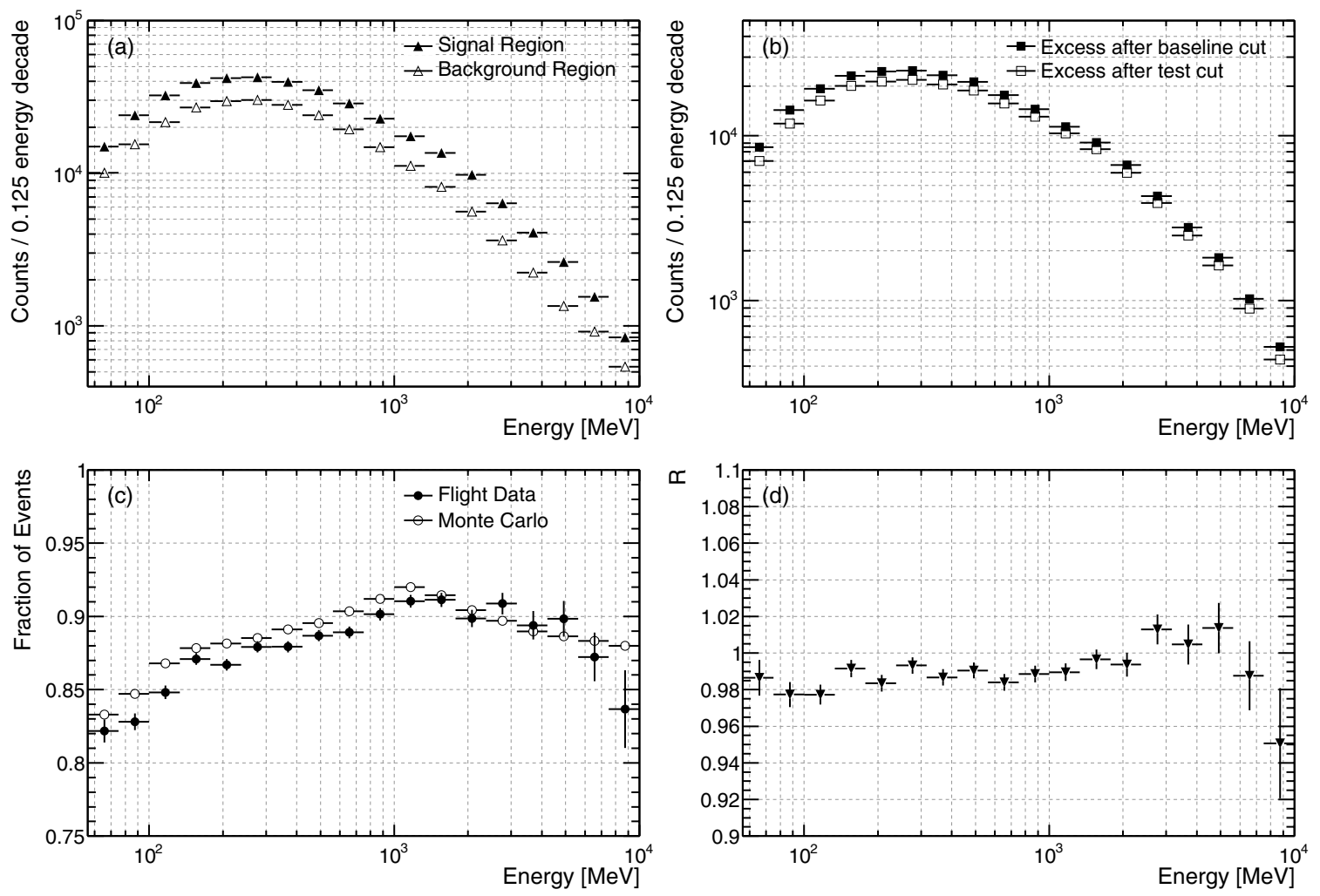

Figure 38. Measurement of the efficiency of a cut using the background subtraction technique: (a) count spectra in the signal and background regions for the flight data set-note that the background counts have been scaled by $1 / 2$ to account for the different phase ranges of the two regions, (b) excess in the signal region before and after the application of the test cut for the flight data sets - note that, for both (a) and (b), the corresponding plots for the MC data sets are not shown, (c) efficiency of the test cut $\eta$ for the flight and MC data sets, (d) ratio $R$ of the efficiencies as measured with flight data to the MC predictions. In this case, the baseline sample is the P7SOURCE event selection on the Vela calibration sample and the cut being tested is the P7CLEAN event selection.

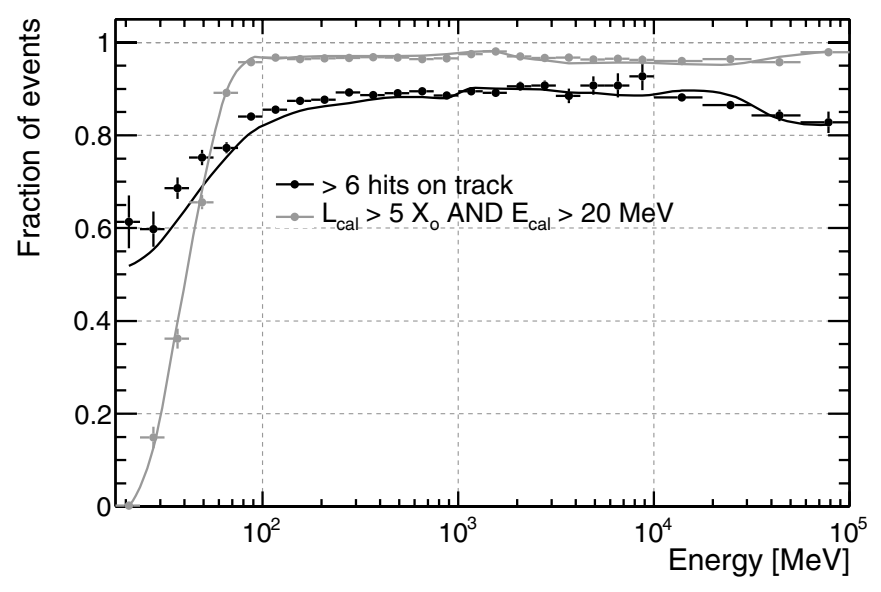

Figure 39. Validation of the track-finding and fiducial cuts. The data points show the fraction of P7SOURCE events in the Vela $(<10 \mathrm{GeV})$ and Earth limb $(>10 \mathrm{GeV})$ calibration samples that pass the fiducial cuts with some margin: having more than the absolute minimum number of hits on a track (black points), or having more than $20 \mathrm{MeV}$ of energy deposition and crossing more than 5 radiation lengths in the CAL (gray points). The curves show the MC predictions for comparison.

Most events that fail these cuts have either poorly reconstructed directions, poorly reconstructed energies, or both-which makes it difficult to study the performance as a function of energy and direction. Therefore, we choose to study the efficiency of the track-finding and fiducial cuts by selecting events that almost fail these cuts in the P7SOURCE calibration samples. Specifically, we study the fraction of events that are very close to the cut thresholds and verify that the flight data agree with our MC simulations, see Figure 39.

\subsubsection{Trigger Conditions and Trigger Request Efficiency}

The LAT hardware trigger, trigger configuration, and onboard filter are described in Section 3.1. For our purpose here we first consider the fractions of $\gamma$-ray events that have one of the five physics trigger conditions (TKR, ROI, CAL_LO, CAL_HI, CNO) asserted. Furthermore, only two of them (TKR and CAL_HI) effectively serve to initiate a trigger request. Of the others, ROI does not exist without TKR, CAL_LO alone does not open the trigger window and from the point of view of selecting $\gamma$-ray events CNO is primarily a veto rather than a trigger. Since the CAL_HI requires at least $1 \mathrm{GeV}$ in a CAL channel, and our fiducial cuts and quality cuts require at least one track, we are effectively using the TKR condition as the primary trigger for $\gamma$ rays up to such energies that the CAL_HI is very likely to be asserted.

Using the DIAGNOSTIC filter events described in Section 3.1, we can measure the fractions of all trigger requests that have individual primitives asserted. However, because of the high particle background rates this does not really probe the trigger stage of the $\gamma$-ray selection process. On the other hand, for each of the five relevant trigger primitives we measure this efficiency as a function of energy for the allGamma sample as well as the P7TRANSIENT selection. For the P7TRANSIENT selection, we also measure the fractions of events with each trigger primitive asserted and compare these to the MC predictions. These are shown in Figure 40. The only notable discrepancy is that the MC overpredicts the fraction of events having ROI 

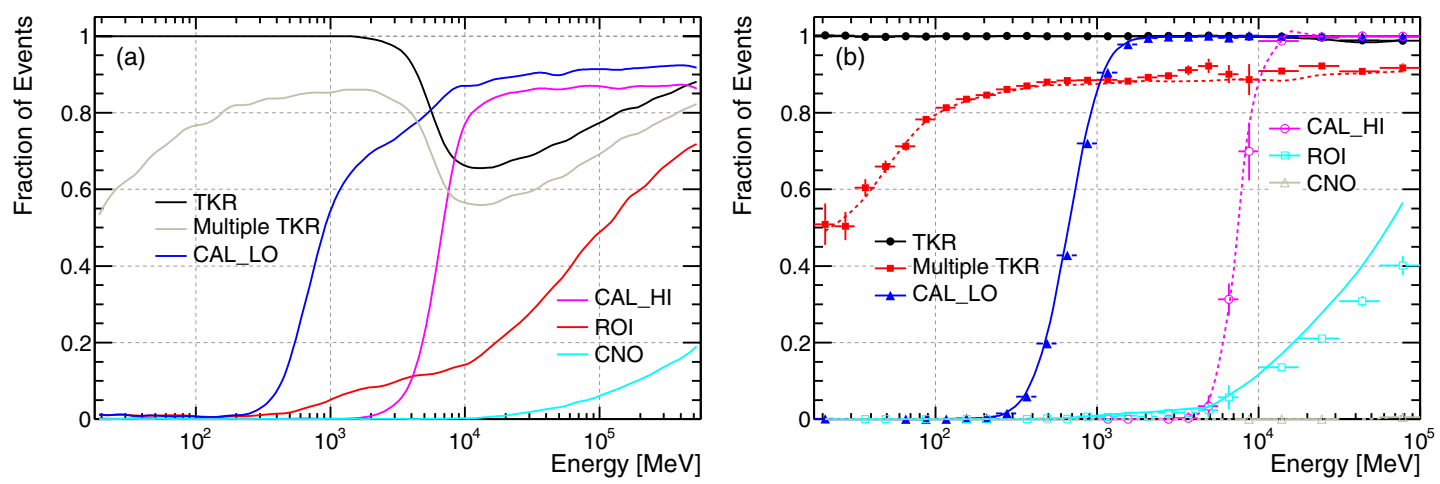

Figure 40. (a) Fractions of all triggered events with various trigger conditions asserted as a function of the energy of the simulated event and (b) fraction of all P7TRANSIENT events that have the same conditions asserted as a function of the reconstructed energy of the event, for MC simulations (lines) and flight data (points). "Multiple TKR" refers to the events with more than one three-in-a-row combinations asserted.

(A color version of this figure is available in the online journal.)

asserted at very high energies; this is likely related to imperfect simulation of the backsplash from the CAL, and since the CAL_LO and CAL_HI are typically asserted for these events, it does not affect the trigger readout decision (see Table 3).

1. The CAL_LO trigger condition requires $100 \mathrm{MeV}$ of energy to be deposited in any CAL channel. This condition starts to be asserted for $\gamma$ rays at $\sim 300 \mathrm{MeV}$ and reaches full efficiency for events contained within the CAL at $\sim 1 \mathrm{GeV}$.

2. The CAL_HI trigger condition requires that $1 \mathrm{GeV}$ of energy be deposited in any CAL channel. This condition starts to be asserted for $\gamma$ rays at $\sim 3 \mathrm{GeV}$ and reaches full efficiency at $\sim 15 \mathrm{GeV}$.

3. The CNO condition requires any ACD tile to have a very large signal, consistent with the passage of a heavy ion. The CNO condition actually serves more to veto than to select an event for readout, but only becomes active at very high energies (>100 GeV), where CAL_LO and CAL_HI are already active.

4. The ROI condition requires any ACD tile in a predefined region of interest associated with a TKR tower to have a signal above 0.45 MIP. The ROI condition actually serves more to veto than to select an event for readout and becomes active at a few $\mathrm{GeV}$.

5. Since the TKR condition serves as the primary trigger, it is very difficult to measure the efficiency of the condition. However, we can estimate how well the MC simulates this efficiency by studying how well it models cases where the events almost fail the trigger request conditions. This is possible by calculating the fraction of events with exactly one combination of three hit layers in a row in the TKR. Most high-energy $\gamma$ rays have many such combinations and would have triggered the LAT even if one hit had been lost. However, for back-converting low-energy events $(<100 \mathrm{MeV})$ the fraction of single combination events becomes significant.

Since the fiducial cuts for our standard event selection require that a track is found, and that the track extrapolates into the $\mathrm{CAL}$, the interplay between the trigger and the standard event selections are actually quite simple.

1. At very low energies, the LAT trigger starts to become efficient at $\sim 10 \mathrm{MeV}$ and follows the efficiency of the TKR condition, which becomes fully efficient by $\sim 100 \mathrm{MeV}$.

2. At around $1 \mathrm{GeV}$ the CAL_LO condition becomes active. By design, this is considerably lower than the $10 \mathrm{GeV}$ where the ROI starts to be asserted because of backsplash.
3. Above $\sim 10 \mathrm{GeV}$ the CAL_HI condition becomes active and we no longer rely on the TKR condition as the primary driver of the trigger.

Furthermore, events that are rejected because ROI is asserted are extremely unlikely to pass standard event class selections. Taken together, this means that the only part of the LAT energy band where the trigger has strong influence on $A_{\text {eff }}$ is below $\sim 100 \mathrm{MeV}$.

\subsubsection{On-board Filter Efficiency}

Although the GAMMA filter has many different cuts (see Section 3.1.2), most events that are rejected by the GAMMA filter would be either rejected by the fiducial cuts (see Section 3.4.1) or by the P7TRANSIENT event selection. Accordingly, we choose to study the efficiency of the GAMMA filter as a whole, and only for those events which pass all the other selection criteria for the P7TRANSIENT class event sample.

Since we downlink a small fraction of the events that fail the GAMMA filter (see Section 3.1), we can check that this is indeed the case for this diagnostic sample. However, the large prescale factor (250) for the DIAGNOSTIC filter and high level of background rejection in the P7TRANSIENT selection severely limit our statistics for this study, and we can do little more than confirm that the GAMMA filter is highly efficient for events in the P7TRANSIENT sample (see Figure 41).

\subsubsection{P7TRANSIENT Class Efficiency}

Measuring the efficiency of the selection for the P7TRANSIENT event class is the most technically challenging part of the $A_{\text {eff }}$ validation for two reasons: (1) at the output of the GAMMA filter the background rates are still great enough to overwhelm almost all traces of $\gamma$-ray signals and (2) the event analysis directs events that are tagged as likely due to CRs away from the remainder of the $\gamma$-ray selection criteria, which means that many of the CT analyses are never applied, and cannot be used to construct a cleaner sample on which we can measure the efficiency of any of these cuts independently of the other cuts.

Figure 42(a) shows the efficiency of each part of the P7TRANSIENT selection on events from the allGamma sample that have passed the GAMMA filter. As stated above, the high levels of CR background make it infeasible to use flight data to obtain stringent constraints on the efficiency of these selection criteria. Therefore, similar to what we did for the track-finding and fiducial cuts, we also study the events that almost fail these 


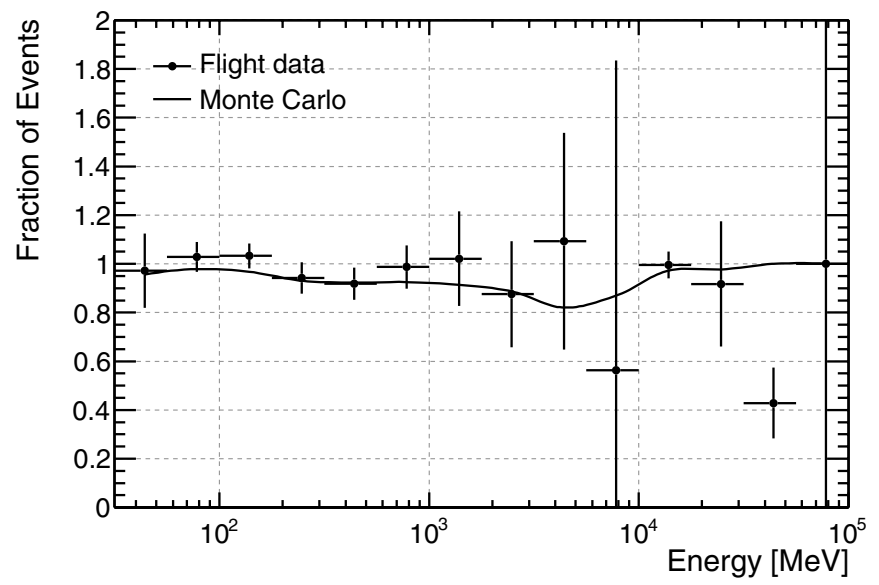

Figure 41. Fraction of events passing the P7TRANSIENT selection that also pass the GAMMA filter for the DIAGNOSTIC filter flight data compared to the MC predictions. Note that point at $42 \mathrm{GeV}$ is well above the $20 \mathrm{GeV}$ high-energy pass criteria where the GAMMA filter becomes fully efficient, so the low measured value of the efficiency is likely due to a statistical fluctuation in the subtracted background.

cuts in the P7TRANSIENT calibration samples: these comparisons are shown in Figure 42(b).

Because of the difficulty in validating the efficiency of the P7TRANSIENT event selection criteria, we have chosen to use the consistency checks described in Section 5.5 to estimate the systematic uncertainty in our $A_{\text {eff }}$ representation.

\subsubsection{P7SOURCE, P7CLEAN, and P7ULTRACLEAN Class Efficiencies}

Although the P7TRANSIENT event class is dominated by residual background across the entire LAT energy range, the background levels are at least reduced to the point where the $\gamma$-ray signals in the calibration samples described in Section 3.6 are clearly detectable. This makes the validation of the effective area from this point on much easier. We can compare the efficiency of each cut, as measured on flight data, to the $\mathrm{MC}$ prediction with the method described in Section 5.3.1. These comparisons are shown in Figure 43.

\subsection{In-flight Effective Area}

We observed that the efficiency for one part of our Pass 6 event selection was systematically lower, near $10 \mathrm{GeV}$ for flight data, than for the allGamma we used to evaluate the effective area, and we attempted to correct the effective area tables to provide more accurate flux measurements for $\gamma$-ray sources. To be more specific, the offending cut is the Pass 6-equivalent of the P7SOURCE cut on the quality of direction reconstruction described in Section 3.4.3 (item 4 in the numbered list). It is important to note that for Pass 7 we chose instead to make this particular cut less stringent to avoid the need to make such a correction, so that the in-flight corrections discussed here do not apply to the effective area tables for the Pass 7 standard $\gamma$-ray classes. Furthermore, we traced the discrepancy to limitations in the pre-launch calibration algorithm of CAL light asymmetry that resulted in degraded position and direction resolution in the CAL: above $\sim 1 \mathrm{GeV}$ consistency between the TKR and CAL position and direction measurements is a strong indicator of accurate direction reconstruction.

We measure the ratio of cut efficiency between flight data and $\mathrm{MC}$ as a function of energy and incidence angle (see Figure 44) - as described in Section 5.3.1—and we use it to correct the MC-based $A_{\text {eff }}$. Because most of our calibration sources have limited statistics relative to the allGamma samples we are forced to use fewer bins when calculating the efficiency ratios. Furthermore, to avoid inducing sharp spectral features in measurements of $\gamma$-ray sources, we smooth the energy and angle dependence of the efficiency ratio. Specifically, we split the data into two $\cos (\theta)$ bins: $[0.2,0.7]$ and $[0.7,1.0]$. For values between the bin centers (i.e., $\cos (\theta) \in[0.45,0.85]$ ), we perform a linear interpolation in $\cos (\theta)$. For values outside that range, we use the correction factor from the appropriate $\cos (\theta)$ bin.

This procedure yields a correction map that we use to convert from the MC $A_{\text {eff }}$ to our best estimate of the true in-flight $A_{\text {eff }}$ for the P6_V11_DIFFUSE set of IRFs, as shown in Figure 45. Note that these IRFs should only be used together with the corresponding rescaled models for Galactic and isotropic diffuse emission provided by the FSSC. ${ }^{83}$

\subsection{Consistency Checks}

In this section, we will describe several consistency checks we performed to estimate how well we understand $A_{\text {eff }}$. Each of these tests consists of splitting a specific $\gamma$-ray event selection into two subsets and comparing the fraction in each of the subsets from the flight data to the predictions from MC simulations.

\footnotetext{
83 Specifically, gll_iem_v02_P6_V11_DIFFUSE.fit and isotropic_iem_v02_P6_V11_DIFFUSE.txt, available at http://fermi.gsfc.nasa.gov/ssc/data/access/lat/BackgroundModels.html.
}
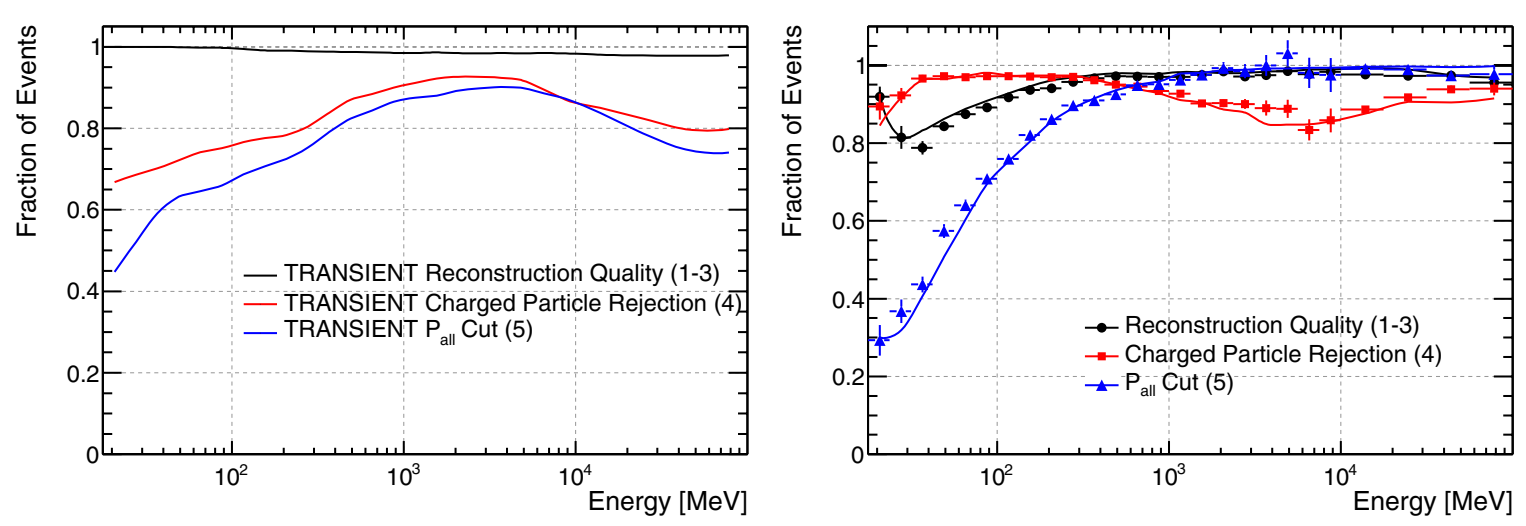

Figure 42. (a) Fractions of events in the allGamma sample passing the GAMMA filter, track-finding, and fiducial cuts that also pass each part of the P7TRANSIENT (Section 3.4.2) event selection. (b) Fractions of events in the P7TRANSIENT calibration samples that pass similar cuts with some margin for MC simulations (lines) and flight data (points). The numbers in the legends refer to the list of cuts in Section 3.4.2.

(A color version of this figure is available in the online journal.) 

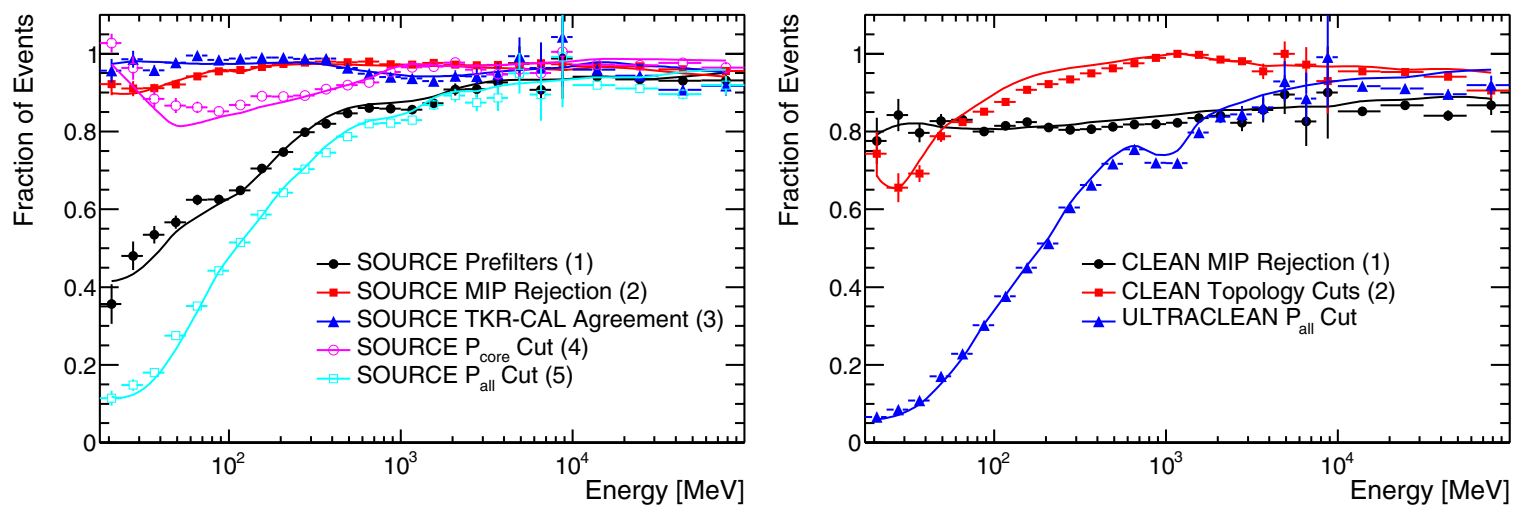

Figure 43. Fractions of events in the P7TRANSIENT calibration samples that pass each part of the (a) P7SOURCE event selection (Section 3.4.3) and (b) P7CLEAN (Section 3.4.4) and P7ULTRACLEAN (Section 3.4.5) event selections for MC simulations (lines) and flight data (points). The numbers in the legends refer to the list of cuts in Sections 3.4.3 and 3.4.4.

(A color version of this figure is available in the online journal.)
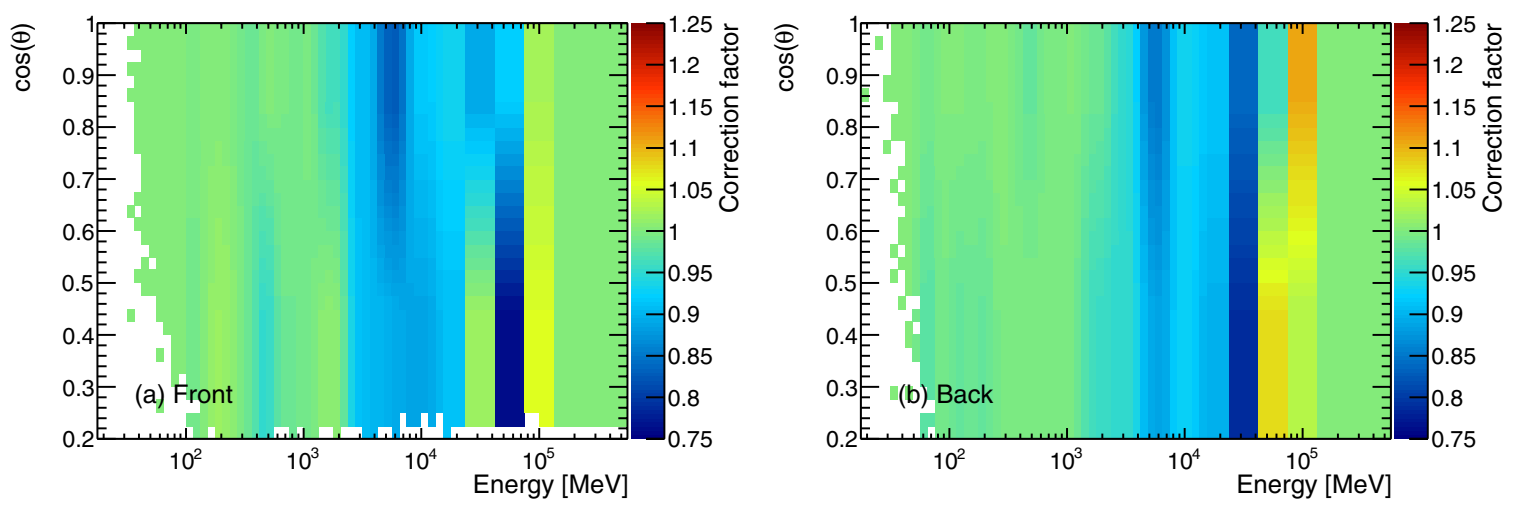

Figure 44. Ratio of the P6_V11_DIFFUSE to P6_V3_DIFFUSE $A_{\text {eff }}$ for front-converting (a) and back-converting (b) events. The P6_V11_DIFFUSE $A_{\text {eff }}$ tables have a correction factor relative to P6_V3_DIFFUSE that is based on the ratio of the efficiencies between flight and simulated data for the selection cut on the direction reconstruction quality (see item 4 in Section 3.4.3). The underflow bins (white areas) had no $A_{\text {eff }}$ in P6_V3_DIFFUSE. As we did not interpolate the correction factor along the energy axis, fluctuations in the correction factor lead to the vertical bands visible in this figure.

(A color version of this figure is available in the online journal.)

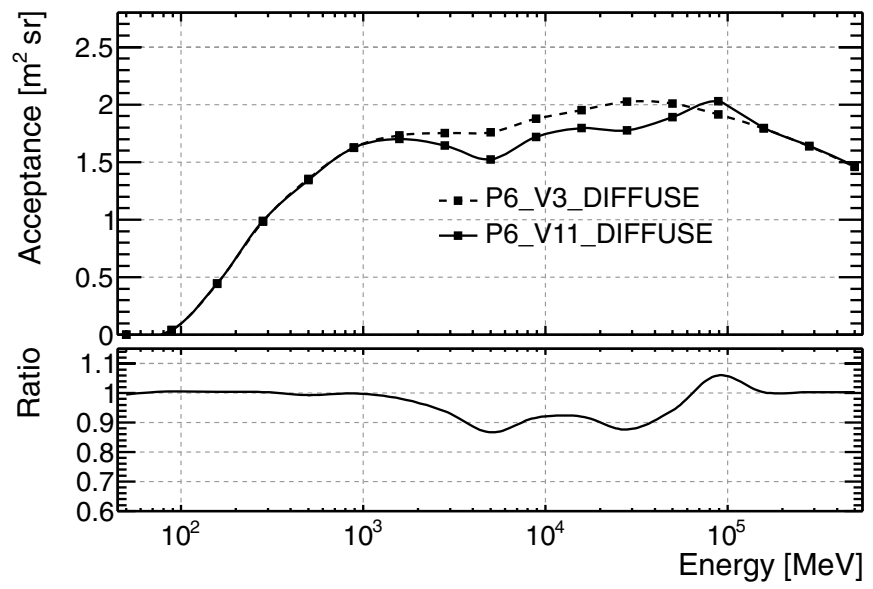

Figure 45. Comparison of acceptance between P6_V3_DIFFUSE and P6_V11_DIFFUSE IRFs. The only difference between the IRFs is the application of the corrections to $A_{\text {eff }}$ for P6_V11_DIFFUSE described in Section 5.4.

As a simple check of our method, we split the event sample into subsets of events converting the $+x$ and $-x$ sides of the LAT. As noted in Section 5.2, the $\phi$ dependence of $A_{\text {eff }}$ is the strongest between directions toward the corners of the LAT relative to directions toward the sides of the LAT; accordingly, we split the data into events coming from the sides $(\xi<0.5)$ or corners $(\xi>0.5)$, based on Equation (15). Finally, we tested the $\theta$ dependence of the $A_{\text {eff }}$ by splitting the data into on-axis $(\cos \theta>0.7)$ and off-axis $(\cos \theta<0.7)$ subsets. The results of these tests are shown in Figure 46. In each of these examples, we used the P7SOURCE event sample as the starting point.

We also compare the fluxes we measure with different event classes. By doing so, we can check the accuracy of our measured efficiency loss for each of the selection cuts to go from one event class to the next. Technically, we do this by asking what fraction of events in one event class also remain into an event class with tighter selection. Figure 46 also shows the results of comparing the P7CLEAN selection to the P7SOURCE selection.

We also compared the fluxes we measure with frontconverting events relative to back-converting events (Figure 47). Since the primary difference between these two parts of the LAT is in the distribution of conversion material, this test is especially sensitive to issues with our Geant 4 simulation and probes our modeling of the trigger and track-finding efficiency.

In each case, we find that the fraction of events in each subset for the flight data are consistent with MC predictions to better than $15 \%$. In fact, for most of the cases the agreement is far better than that, closer to the $2 \%-3 \%$ level. The most significant discrepancies we see are between front- and back-converting events (Figure 47). 

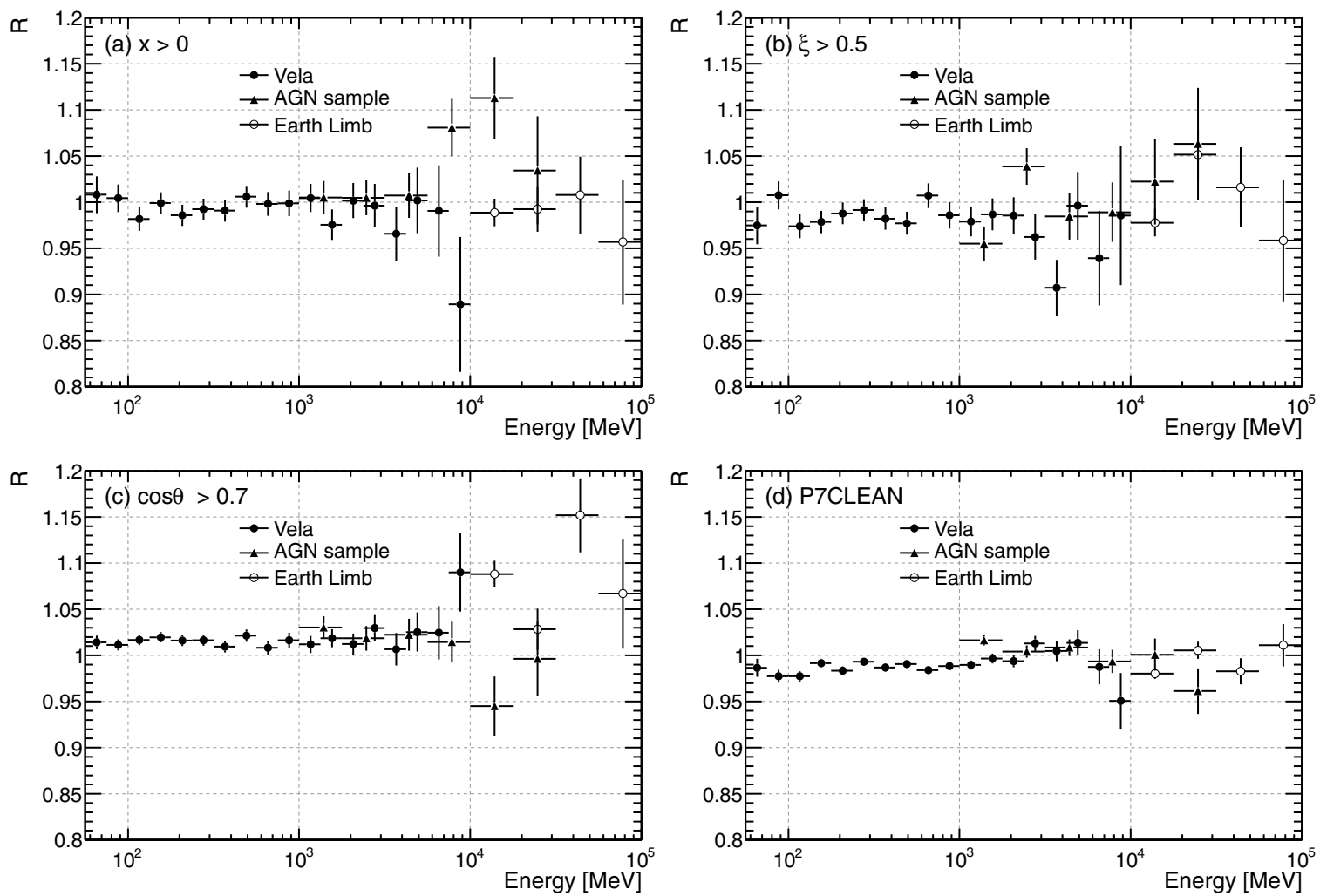

Figure 46. Ratio of the fraction of events as seen in flight data relative to the prediction from MC simulations: (a) $\gamma$ rays converting on the $+x$ side of the LAT, (b) $\gamma$ rays pointing in $45^{\circ}$ ranges of azimuth toward the corners of the LAT (i.e., $\xi>0.5$ ), (c) $\gamma$ rays from near the LAT boresight, i.e., for which cos $\theta>0.7$, (d) $\gamma$ rays passing the P7CLEAN selection.

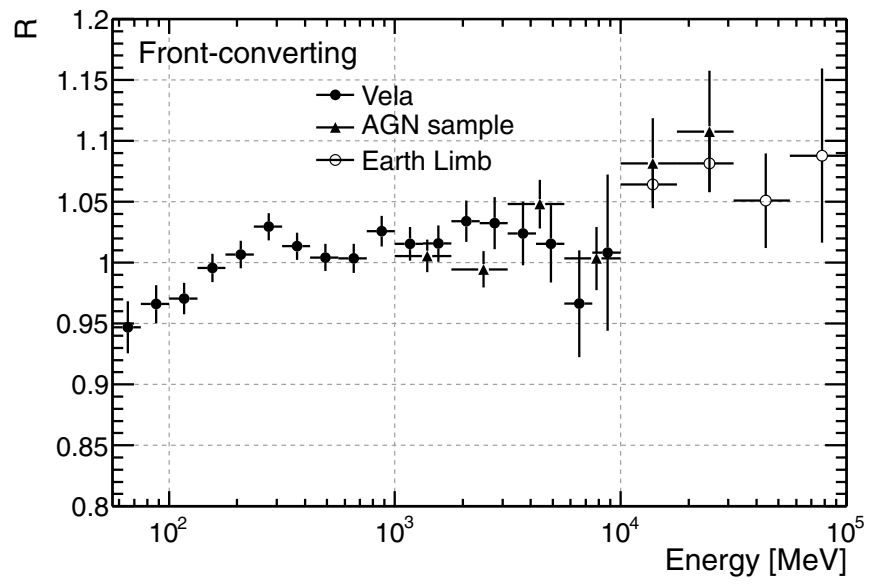

Figure 47. Ratio of the fraction of front-converting events as seen in flight data relative to the prediction from MC simulations.

\subsection{Uncertainties on the Effective Area}

\subsubsection{Overall Uncertainty of the Effective Area}

From the consistency checks described in the previous section, we arrive at a rough overall estimate of the uncertainty of $A_{\text {eff }}$, which is shown in Figure 48. Note that this estimate is assigned simply to account for the largest observed inconsistency, namely the mismatch between the front- and backconverting events. Roughly speaking, for the P7SOURCE_V6 and P7CLEAN_V6 event classes these uncertainties may be quoted as $10 \%$ at $100 \mathrm{MeV}$, decreasing to $5 \%$ at $560 \mathrm{MeV}$, and increasing to $10 \%$ at $10 \mathrm{GeV}$ and above. It is important to note that these uncertainties are statements about overall uncertainty of $A_{\text {eff }}$ at

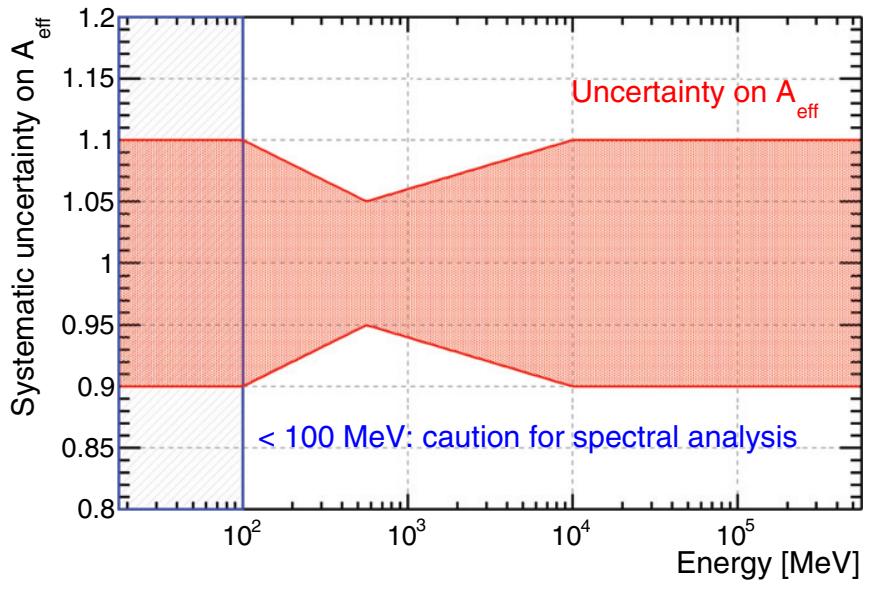

Figure 48. Systematic uncertainty band on $A_{\text {eff }}$ as a function of energy. The interplay between the steeply decreasing effective area and the degrading energy resolution below $100 \mathrm{MeV}$ and the resulting impact on spectral analysis will be thoroughly discussed in Section 7.

(A color version of this figure is available in the online journal.)

various energies, and do not include any statement about what types of deviations we might expect within the stated uncertainty bands, nor about the point-to-point correlations in any systematic biases of $A_{\text {eff }}$. Those questions are addressed in the next sections.

\subsubsection{Point-to-point Correlations of the Effective Area}

Since our selection criteria are generally scaled with energy (see Section 3.3.1), we expect any biases of $A_{\text {eff }}$ to be highly correlated from one energy band to the next. This point is very 

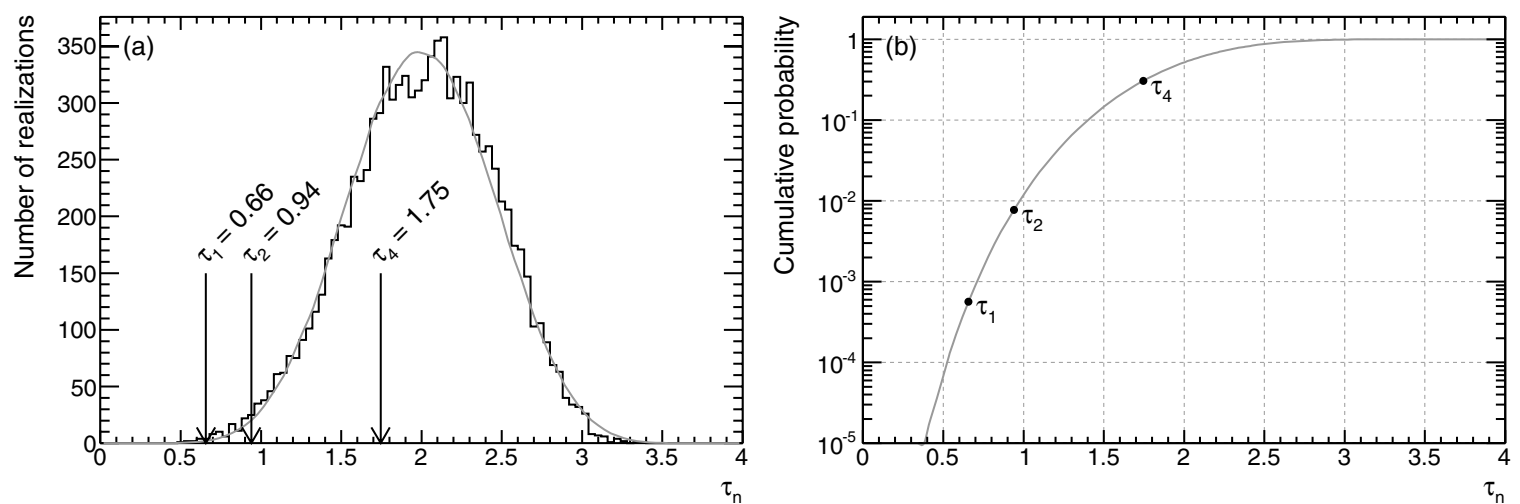

Figure 49. (a) Values of $\tau_{n}(n=1,2$, and 4) defined in Equation (21) for the front/back consistency check with the Vela data set shown in Figure 47. The parent distribution of $\tau_{n}$ for random normal uncorrelated deviations is shown in gray, while the black histogram represents the values of $\tau_{n}$ for 10,000 random permutations of the original data points. (Note that in both cases the distributions are independent of $n$.) (b) Cumulative probability distribution for random normal uncorrelated deviations.

important when estimating the size of potential instrumental spectral artifacts.

We have studied the point-to-point correlation of the effective area through the consistency checks described in Section 5.5, where it is evident that the deviations from unity are not independent for neighboring energy bins. In order to quantify this correlation we first scale the values $r_{i}$ of the data-to-MC ratio $R$ in each of the $N$ energy bins (indexed by $i$ ), turning them into normalized deviations

$$
d_{i}=\frac{\left(r_{i}-m\right)}{\sqrt{\frac{1}{(N-1)} \sum_{i=1}^{N}\left(r_{i}-m\right)^{2}}},
$$

and $m$ is the arithmetic mean of the $r_{i}$ ) and then we construct the metrics

$$
\tau_{n}=\frac{\sum_{i=1+n}^{N}\left(d_{i}-d_{i-n}\right)^{2}}{(N-n)} .
$$

The quantity in Equation (21) is related to a reduced $\chi_{N-n}^{2}(2 x)$; it is small for highly positively correlated deviations (in which case the differences between neighboring bins are generally small), while the expectation value is 2 , for all values of $n$, for normal uncorrelated errors.

Figure 49 shows this metric, for different values of $n$, calculated on the front versus back consistency check shown in Figure 47. (Only the Vela calibration data set is considered here.) The extremely small value of $\tau_{1}$ indicates that neighboring logarithmic energy bins are highly positively correlated (as can be naively inferred from the plot in Figure 47), while on the scale of half a decade in energy $(n=4)$ there is little evidence of a correlation. This implies that the systematic uncertainties on the effective area are not likely to introduce significant spectral features over scales much smaller than half a decade in energy (which is much larger than the LAT energy resolution). The results for all the consistency checks are summarized in Table 8.

For many analyses, especially those that are limited by statistics, it is enough to consider the overall uncertainty and allow for worst case deviations within the stated uncertainty bands. However, doing so will result in very conservative systematic error estimates. We will discuss this in more detail in Section 5.7 when we describe techniques to propagate the estimates of the uncertainty of $A_{\text {eff }}$ to uncertainties on quantities such as fluxes and spectral indices. Furthermore, we will come back to the issues of point-to-point correlations and the potential induced spectral features when we discuss the uncertainties associated with the energy reconstruction in Section 7.
Table 8

Summary of the Point-to-point Correlations for the Consistency Checks Described in Section 5.5

\begin{tabular}{lcc}
\hline \hline Consistency Check & $\tau_{1}$ & $P\left(\tau<\tau_{1}\right)$ \\
\hline$+x$ vs. $-x$ & 1.24 & $4.9 \times 10^{-2}$ \\
Azimuthal dependence & 2.21 & 0.69 \\
$\theta$ dependence & 1.05 & $1.6 \times 10^{-2}$ \\
P7CLEAN selection & 0.95 & $8.4 \times 10^{-3}$ \\
Front vs. back & 0.66 & $5.0 \times 10^{-4}$ \\
\hline
\end{tabular}

Note. Most of them indicate a strong positive correlation of the systematic biases between adjacent bins on the effective area (the expectation value for random normal uncorrelated deviations is $\tau_{n}=2$ for all values of $n$ ).

\subsubsection{Variability Induced by Errors in the Effective Area}

As a source moves across the LAT FoV and $A_{\text {eff }}$ changes with the viewing angle, any errors in the $A_{\text {eff }}$ parameterization as a function of $\theta$ potentially could induce artificial variability. We have searched for such induced variability with Vela. We split the data set into $12 \mathrm{hr}$ periods (indexed by $i$ ) and compared the number of $\gamma$ rays observed $\left(n_{i}\right)$ during each period with the number of $\gamma$ rays we predict $\left(\tilde{n}_{i}\right)$ based on the fraction of the total exposure for Vela that we integrated during that $12 \mathrm{hr}$ period.

On average, our Vela calibration sample contains 230 (176) on-peak (off-pulse) P7SOURCE class events in the $100 \mathrm{MeV}$ to $10 \mathrm{GeV}$ energy band every $12 \mathrm{hr}$. Since the off-pulse region is twice the size of the on-peak region, background subtraction yields an average on-peak excess of $n_{i}=142 \gamma$ rays with an average statistical uncertainty of $\sigma_{i}=16 \gamma$ rays in each time interval.

The exposure calculation requires several points of input.

1. The spacecraft pointing and live time history, which are binned in $30 \mathrm{~s}$ intervals.

2. The P7SOURCE_V6 $A_{\text {eff }}$ parameterization, which we use when deriving $A_{\mathrm{eff}}(E, t)$, the effective area for $\gamma$ rays for Vela for each $30 \mathrm{~s}$ interval.

3. The P7SOURCE_V6MC PSF (see Section 6.1) parameterization, which allows us to calculate the energy-dependent containment $C\left(E, t, 15^{\circ}\right)$ within the $15^{\circ}$ ROI for each $30 \mathrm{~s}$ interval.

4. A parameterization of the spectrum of Vela $F(E)$ so that we may correctly integrate $A_{\mathrm{eff}}(E, t)$ and $C\left(E, t, 15^{\circ}\right)$ over the energy range. 

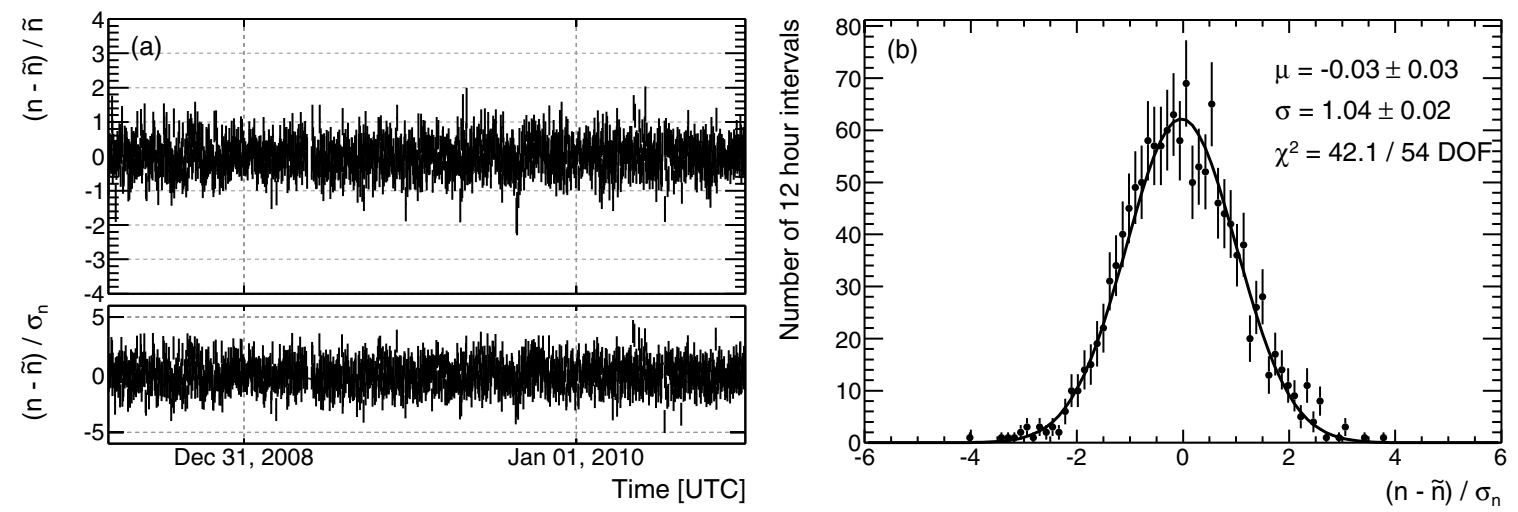

Figure 50. Check for variability induced by errors in the $A_{\text {eff }}$ tables P7SOURCE Vela calibration sample and the P7SOURCE_V6MC IRFs. Panel (a) shows the fractional difference and normalized residuals between the observed counts in the $100 \mathrm{MeV}$ to $10 \mathrm{GeV}$ band and the prediction based on the fraction of the total exposure accumulated during each of the $140012 \mathrm{hr}$ time intervals. Panel (b) shows the normalized residuals, which are very well fit with a Gaussian with unit width and zero mean.

In order to minimize dependence on the modeled flux, we calculate the exposure independently for 24 energy bins (which we index by $j$ ). The exposure in a single time and energy bin is

$$
\mathcal{E}_{i, j}=\int^{E_{i}} \int^{t_{j}} A_{\mathrm{eff}}(E, t) C\left(E, t, 15^{\circ}\right) \frac{F(E)}{\int^{E_{i}} F(E) d E} d t d E .
$$

We can then express the expected number of $\gamma$ rays in each time and energy bin $\left(\tilde{n}_{i, j}\right)$ as a fraction of the total number of $\gamma$ rays in that energy bin $\left(n_{j}\right)$,

$$
\tilde{n}_{i, j}=\frac{\mathcal{E}_{i, j}}{\sum_{i} \mathcal{E}_{i, j}} n_{j}
$$

Then we sum $n_{i, j}$ and $\tilde{n}_{i, j}$ across energy bins to find $n_{i}$ and $\tilde{n}_{i}$.

We have performed this analysis, dividing the first 700 days of the Vela data sample into $140012 \mathrm{hr}$ time intervals and using the phase-averaged flux model

$$
F(E) \propto E^{-\Gamma} e^{-\frac{E}{E_{0}}}
$$

with $\Gamma=1.38$ and $E_{0}=1.360 \mathrm{GeV}$ (Abdo et al. 2009e). Figure 50 shows the fractional variation,

$$
\frac{n_{i}-\tilde{n}_{i}}{\tilde{n}_{i}}
$$

the normalized residuals,

$$
\frac{n_{i}-\tilde{n}_{i}}{\sigma_{i}}
$$

and the distribution of the normalized residuals for each of the time intervals. The normalized residuals are very nearly normally distributed. Furthermore, the Fourier transformation of the time series (Figure 51) shows only a small peak corresponding to the orbital precession period and is otherwise consistent with Poisson noise. Note that unlike more complicated analyses that involve fitting the flux of a point source, this analysis tests only the accuracy of the $A_{\text {eff }}$ representation (and to a much lesser extent, the $15^{\circ}$ containment of the PSF). We attribute the peak in the Fourier spectrum to small, incidence angle-dependent errors in the effective area. As the orbit precesses, the range of

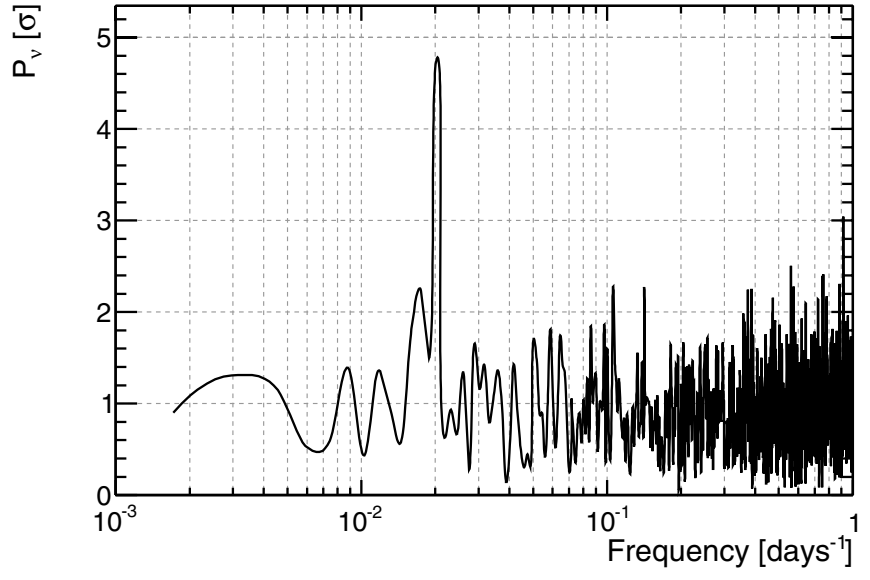

Figure 51. Discrete Fourier transform of the $(n-\tilde{n}) / \sigma_{n}$ time series. The only peak visible above the noise floor corresponds to the 53 day orbital precession period. Note that the figure is normalized and the vertical scale is expressed in units of the statistical uncertainty.

incidence angles sampled, and hence the bias in the calculated exposure, varies slightly.

Although we performed this analysis with $12 \mathrm{hr}$ time intervals, as noted in Section 2.3, the LAT boresight follows very similar paths across the sky during successive two-orbit periods. Therefore, the level of instrument-induced variability observed with $12 \mathrm{hr}$ time intervals is likely to be indicative of the systematic uncertainties for variability analyses down to $3 \mathrm{hr}$ timescales.

Although the estimate used in Section 5.6.1 that the systematic uncertainty of $A_{\text {eff }}$ is less than the disagreement between the extreme cases is quite conservative for long timescale observations, it is somewhat less conservative for shorter observations. For example, in observations less than the Fermi orbital precession period of $\sim 53.4$ days, a particular region of the sky might be preferentially observed at incidence angles where the bias of $A_{\text {eff }}$ is particularly large, or during parts of the orbit in which Fermi is exposed to particularly high CR background rates and the correction described in Section 5.2.2 leaves some residual bias in the calculated exposure. Finally, we have observed that ignoring the $\phi$ dependence of the effective area (Section 5.2.3) can induce artificial quarterly periodicity in the fluxes from directions near extremely bright sources, in particular the Vela pulsar. 

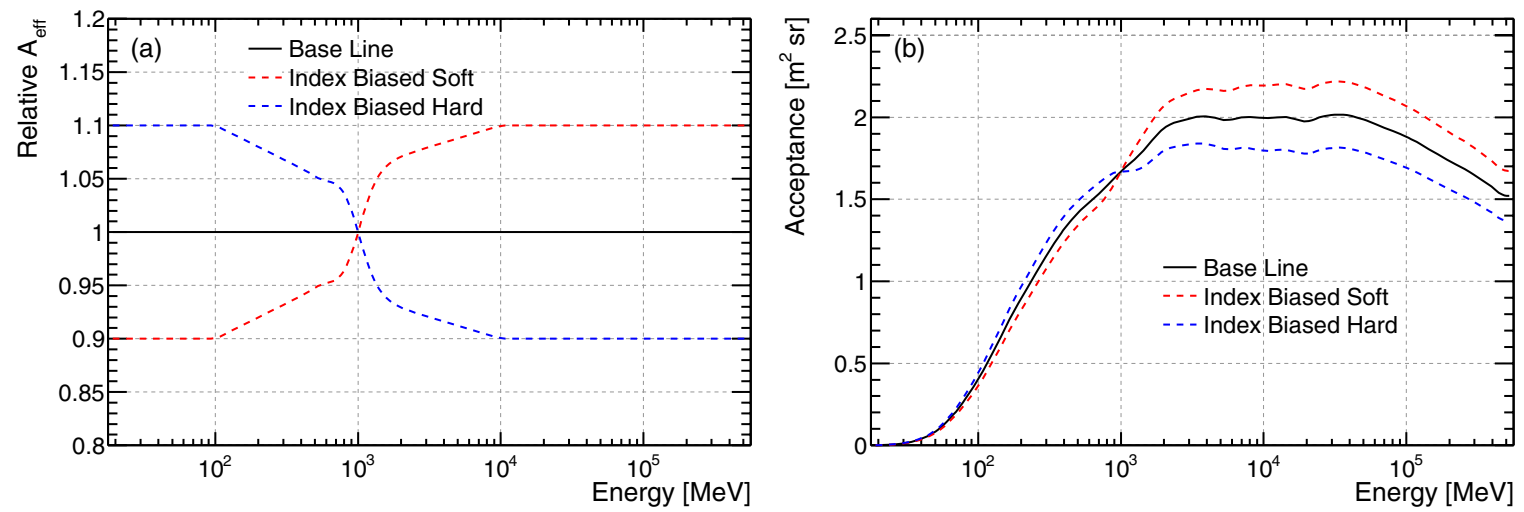

Figure 52. Bracketing IRFs for $A_{\text {eff }}$ designed to estimate possible systematic biases of the measurement of the spectral index. (a) Energy dependence of the scaling parameter $\epsilon(E) B(E)$. (b) Acceptance of the resulting scaled $A_{\text {eff }}$ for P7SOURCE. For this example, we used a pivot energy $E_{0}=1 \mathrm{GeV}$.

(A color version of this figure is available in the online journal.)

\subsection{Propagating Uncertainties on the Effective Area to High-level Science Analysis}

As we hinted in the previous section, translating uncertainties on $A_{\text {eff }}$ into systematic errors on quantities such as fluxes and spectral indices depends on the particular analysis and requires assumptions about the variation of $A_{\text {eff }}$ within the uncertainty bands.

\subsubsection{Using Custom-made IRFs to Generate an Error Envelope}

A somewhat brute force approach to this problem is to generate IRFs that represent worst case scenarios for measuring specific quantities like fluxes or spectral parameters and use these bracketing IRFs to repeat the analysis and extract the variation in the measured quantities. Of course, the nature of the variations between the IRFs depends on the quantity in question.

We address this in a generic way by scaling $A_{\text {eff }}$ by the product of the relative systematic uncertainty

$$
\epsilon(E)=\frac{\delta A_{\mathrm{eff}}(E)}{A_{\mathrm{eff}}(E)}
$$

and arbitrary bracketing functions $B(E)$ taking values in the $[-1,1]$ interval. Specifically, we define modified $A_{\text {eff }}$ as

$$
A_{\mathrm{eff}}^{\prime}(E, \theta)=A_{\mathrm{eff}}(E, \theta) \cdot(1+\epsilon(E) B(E)) .
$$

The simplest bracketing functions, $B(E)= \pm 1$ clearly minimize and maximize $A_{\text {eff }}$ within the uncertainty band. On the other hand, to maximize the effect on the spectral index in a power-law fit, we choose a functional form that changes sign at the pivot or decorrelation energy $E_{0}$ (i.e., the energy at which the fitted differential flux and spectral index are uncorrelated):

$$
B(E)= \pm \tanh \left(\frac{1}{k} \log \left(E / E_{0}\right)\right) .
$$

The parameter $k$ controls the slope of the transition near $E_{0}$; in practice we use $k=0.13$, which corresponds to smoothing over twice the LAT energy resolution of $\Delta E / E \sim$ 0.15 . The bracketing IRFs used for effective area studies are listed in Table 9. Figure 52 shows the bracketing functions for $c \_i n d e x \_s o f t$ and $c \_i n d e x \_h a r d$ and their effects on the on-axis $A_{\text {eff }}$.

We have studied the following two sources from the AGN sample to obtain estimates of the effects of instrumental uncertainties on the measured fluxes and spectral parameters.
Table 9

Bracketing $A_{\text {eff }}$ and Corresponding Energy-dependent Scaling Functions Used to Create Them

\begin{tabular}{lc}
\hline \hline Name & $B(E)$ \\
\hline$c \_f l u x \_l o$ & +1 \\
$c \_f l u x \_h i$ & -1 \\
c_index_soft & $+\tanh \left(\frac{1}{k} \log \left(E / E_{0}\right)\right)$ \\
c_index_hard & $-\tanh \left(\frac{1}{k} \log \left(E / E_{0}\right)\right)$ \\
\hline
\end{tabular}

1. PG 1553+113: associated with 2FGL J1555.7+1111, which has a spectral index of $1.66 \pm 0.02$, making it one of the hardest bright AGNs.

2. B2 1520+31: associated with 2FGL J1522.1+3144, which has a spectral index of $2.37 \pm 0.02$ (when fit with a power law), making it one of the softest bright AGNs.

In each case we used ScienceTools (version v9r25p2) to perform a series of binned maximum likelihood fits to a $20^{\circ} \times 20^{\circ}$ region centered at the source position over the energy range $100 \mathrm{MeV}-100 \mathrm{GeV}$. For each individual fit we followed the same procedure.

1. Used the $\gamma$-ray and time interval selection criteria as for the Vela calibration sample (see Section 3.6).

2. Included all $2 \mathrm{FGL}$ sources within $20^{\circ}$ in our likelihood model, with the same spectral parameterizations as were used in the 2FGL catalog.

3. Included models of the Galactic diffuse emission (ring_2year_P76_vo.fits) and the isotropic diffuse emission (isotrop_2year_P76_source_v1.txt) rescaled by the inverse of the function used to rescale the effective area (so as to ensure the same distribution of expected counts from these diffuse sources).

4. Freed the spectral parameters for all point sources within $8^{\circ}$ from the center of the region, as well as the overall normalizations of both diffuse components.

Following what was done for the 2FGL catalog, we used a simple power law

$$
\frac{d N}{d E}=N_{0}\left(\frac{E}{E_{0}}\right)^{-\Gamma}
$$


Table 10

Fit Parameters and Integral Fluxes Obtained Using the $A_{\text {eff }}$ Bracketing IRFs for PG 1553+113

\begin{tabular}{lcccc}
\hline \hline Bracketing $A_{\mathrm{eff}}$ & $\begin{array}{c}N_{0} \\
\left(\mathrm{MeV}^{-1} \mathrm{~cm}^{-2} \mathrm{~s}^{-1}\right)\end{array}$ & $\Gamma$ & $\begin{array}{c}F_{25} \\
\left(\mathrm{~cm}^{-2} \mathrm{~s}^{-1}\right)\end{array}$ & $\begin{array}{c}S_{25} \\
\left(\mathrm{MeV} \mathrm{cm}^{-2} \mathrm{~s}^{-1}\right)\end{array}$ \\
\hline Nominal & $2.54 \times 10^{-12}$ & 1.68 & $6.91 \times 10^{-8}$ & $1.19 \times 10^{-4}$ \\
c_flux_hi & $2.75 \times 10^{-12}$ & 1.67 & $7.32 \times 10^{-8}$ & $1.31 \times 10^{-4}$ \\
c_flux_lo & $2.37 \times 10^{-12}$ & 1.69 & $6.54 \times 10^{-8}$ & $1.09 \times 10^{-4}$ \\
c_index_hard & $2.57 \times 10^{-12}$ & 1.64 & $6.45 \times 10^{-8}$ & $1.30 \times 10^{-4}$ \\
c_index_soft & $2.53 \times 10^{-12}$ & 1.73 & $7.44 \times 10^{-8}$ & $1.11 \times 10^{-4}$ \\
\hline
\end{tabular}

Note. The quoted precision is roughly equivalent to the fit uncertainties and the pivot energy is $E_{0}=2240 \mathrm{MeV}$ for this source.

Table 11

Fit Parameters and Integral Fluxes Obtained Using the $A_{\text {eff }}$ Bracketing IRFs for B2 1520+31

\begin{tabular}{lccccc}
\hline \hline Bracketing $A_{\mathrm{eff}}$ & $\begin{array}{c}N_{0} \\
\left(\mathrm{MeV}^{-1} \mathrm{~cm}^{-2} \mathrm{~s}^{-1}\right)\end{array}$ & $\alpha$ & $\beta$ & $\begin{array}{c}F_{25} \\
\left(\mathrm{~cm}^{-2} \mathrm{~s}^{-1}\right)\end{array}$ & $\begin{array}{c}S_{25} \\
\left(\mathrm{MeV} \mathrm{cm}^{-2} \mathrm{~s}^{-1}\right)\end{array}$ \\
\hline Nominal & $5.23 \times 10^{-10}$ & 2.24 & 0.08 & $4.09 \times 10^{-7}$ & $1.33 \times 10^{-4}$ \\
c_flux_hi & $5.60 \times 10^{-10}$ & 2.26 & 0.07 & $4.44 \times 10^{-7}$ & $1.44 \times 10^{-4}$ \\
c_flux_lo & $4.90 \times 10^{-10}$ & 2.22 & 0.08 & $3.79 \times 10^{-7}$ & $1.24 \times 10^{-4}$ \\
c_index_hard & $5.20 \times 10^{-10}$ & 2.15 & 0.10 & $3.93 \times 10^{-7}$ & $1.35 \times 10^{-4}$ \\
c_index_soft & $5.27 \times 10^{-10}$ & 2.33 & 0.05 & $4.29 \times 10^{-7}$ & $1.32 \times 10^{-4}$ \\
\hline
\end{tabular}

Note. The quoted precision is roughly equivalent to the fit uncertainties and the pivot energy is $E_{0}=281 \mathrm{MeV}$ for this source.

to model the differential flux from PG $1553+113$ and a "logparabola"

$$
\frac{d N}{d E}=N_{0}\left(\frac{E}{E_{0}}\right)^{-\left(\alpha+\beta \ln \left(E / E_{0}\right)\right)}
$$

to model that of B2 $1520+31$. (All of this will also be relevant for the tests with bracketing PSFs described in Section 6.5.1 and with energy dispersion included in the likelihood fit described in Section 7.4.)

Tables 10 and 11 show the fit results for PG $1553+113$ and B2 $1520+31$, respectively, using these $A_{\text {eff }}$ bracketing functions, as well as the integral counts $\left(F_{25}\right)$ and energy $\left(S_{25}\right)$ fluxes between $100 \mathrm{MeV}$ and $100 \mathrm{GeV}$.

The ranges of the fit values indicate propagated uncertainties from the uncertainty in $A_{\text {eff }}$ (Table 12). It is important to note that the systematic error estimates resulting from this technique represent conservative estimates within the instrumental uncertainties, rather than random variations. Furthermore, many of the bracketing IRFs are mutually exclusive, so when considering relative variations between $\gamma$-ray sources it is more appropriate to compare how the relative values change for each set of bracketing IRFs.

\subsubsection{Using a Bootstrap Method to Generate an Error Envelope}

Alternatively, given a family of plausible $A_{\text {eff }}$ curves, we can use a weighted bootstrap approach (see Efron \& Tibshirani 1993, for more details) for propagating the systematic uncertainties on $A_{\text {eff }}$. The weighed bootstrap approach is closely related to the bracketing IRFs method described in the previous section and to the methods discussed in Lee et al. (2011) in the context of the analysis of Chandra X-ray data.

The basic idea is that, for each trial, the event data are bootstrap resampled using a weighting based on an effective area scaling function that is drawn from a family of plausible curves. The simplest of such families of $A_{\text {eff }}$ curves (Figure 53) can be constructed starting from Equation (28) and multiplying the scaling function $\epsilon(E) B(E)$ by a normally distributed random
Table 12

Systematic Variations Arising from Uncertainties in the Effective Area

\begin{tabular}{lcccc}
\hline \hline Parameter & $\begin{array}{c}\text { B2 } 1520+31 \\
\text { (Soft) }\end{array}$ & $\begin{array}{c}\text { PG 1553+113 } \\
\text { (Hard) }\end{array}$ \\
\hline$\delta N_{0} / N_{0}$ & $+7.2 \%$ & $-6.3 \%$ & $+8.0 \%$ & $-6.9 \%$ \\
$\delta \Gamma(\delta \alpha)$ & +0.09 & -0.09 & +0.05 & -0.05 \\
$\delta \beta$ & +0.02 & -0.02 & \multicolumn{2}{c}{$\ldots$} \\
$\delta F_{25} / F_{25}$ & $+8.5 \%$ & $-7.2 \%$ & $+7.7 \%$ & $-6.6 \%$ \\
$\delta S_{25} / S_{25}$ & $+8.1 \%$ & $-6.9 \%$ & $+10.0 \%$ & $-8.3 \%$ \\
\hline
\end{tabular}

Notes. For the spectral index ( $\Gamma$ or $\alpha$ ) and spectral curvature $(\beta)$, we give the absolute variation with respect to the nominal value (e.g., $\delta \Gamma)$. For the flux prefactor and the integral fluxes, we give the relative variation with respect to the nominal value (e.g., $\delta N_{0} / N_{0}$ ).

number $\xi$ with zero mean-which effectively becomes the parameter controlling the family itself:

$$
A_{\mathrm{eff}}^{\prime}(E, \theta, \xi)=A_{\mathrm{eff}}(E, \theta) \cdot(1+\xi \epsilon(E) B(E)) .
$$

We have found the results of the weighted bootstrap with the family of scaling functions defined in Equation (32) to be in good agreement with those of the bracketing IRFs approach described in the previous section. This should not be surprising as the bracketing IRFs use the $\pm 1 \sigma$ excursions of the bracketing function and the weighted bootstrap draws from a Gaussian distribution of function scalings that are also based on that same bracketing function. The real benefits of the weighted bootstrap arise when one has families of plausible effective area functions that have more complicated dependencies (e.g., on incidence angle as well as energy) such that exposures would need to be recalculated to apply the bracketing method.

\subsection{Comparison of Pass 6 and Pass 7}

The Pass 7 event classes were designed to meet the same high-level analysis needs as the Pass 6 classes. Table 5 summarizes the correspondence between the Pass 7 standard $\gamma$-ray 


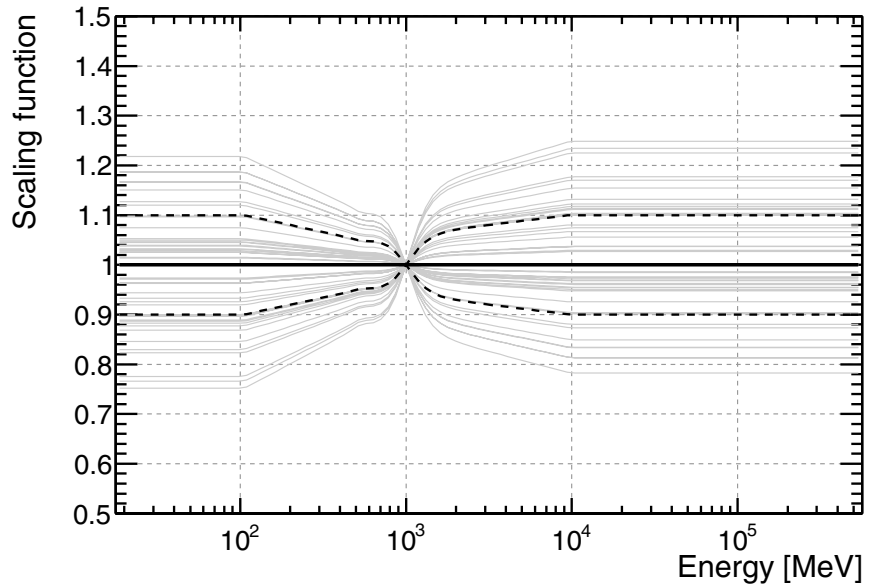

Figure 53. Family of effective area scaling functions obtained for different values of the normal random variable $\xi$ in Equation (32).

classes and their closest Pass 6 equivalents. The corresponding acceptances are shown in Figure 54.

As discussed in Section 3, the main technical improvement in Pass 7 was optimizing the event selections on simulations that included the ghost signals, and on flight data. The principal outcome for Pass 7 is a substantial increase of $A_{\text {eff }}$ below $\sim 300 \mathrm{MeV}$, especially for the cleaner event classes. At $100 \mathrm{MeV}$ the P7SOURCE_V6 and P7CLEAN_V6 $\gamma$-ray classes feature an acceptance of $\sim 0.3 \mathrm{~m}^{2}$ sr, to be compared with the $\sim 0.1 \mathrm{~m}^{2} \mathrm{sr}$ of the Pass 6 equivalent (Figure 54). Pass 7 has opened a window on astronomy with the LAT data below $100 \mathrm{MeV}$, though the reader should bear in mind the specific caveats in Sections 6.5 and 7.4 when performing spectral analyses at these energies.

Additionally, in Pass 7, the energy dependence of $A_{\text {eff }}$ is smoother. This was accomplished in conjunction with obtaining a better overall understanding of the effective area itself, owing to the extensive use of on-orbit data to verify the fidelity of our MC simulation at each step of the $\gamma$-ray selection process.

\section{POINT-SPREAD FUNCTION}

As discussed in Section 2.1.1, at low energies the PSF is determined by multiple scattering. For example, the calculated multiple scattering for a normally incident $100 \mathrm{MeV} \gamma$ ray converting in the middle of a thin (front) section tungsten foil is $\sim 3.1$ (space angle). The $68 \%$ containment angle as measured from flight data for $100 \mathrm{MeV} \gamma$ rays near the LAT boresight averaged over all towers is $\sim 3.3$ and is in agreement with the MC simulations (see Section 6.2). The small difference is due to missing measurements when the trajectories happen to pass through regions without SSD coverage and the fact that the electron and positron from a conversion can undergo hard scattering processes such as bremsstrahlung.

If multiple scattering were the only consideration, the PSF should become narrower as $E^{-1}$. The measured PSF, however, improves more slowly with energy, instead falling as $\sim E^{-0.78}$ (see Section 3.3.3). This slower improvement relative to that expected for pure multiple scattering is also due to missed measurements and hard scattering processes and is predicted by the MC calculations.

Above a few $\mathrm{GeV}$, the narrowing of the PSF with energy is limited by the finite hit resolution of the SSDs. The strip pitch of $228 \mu \mathrm{m}$ and the lever arm for the direction measurement result in a limiting precision for the average conversion of

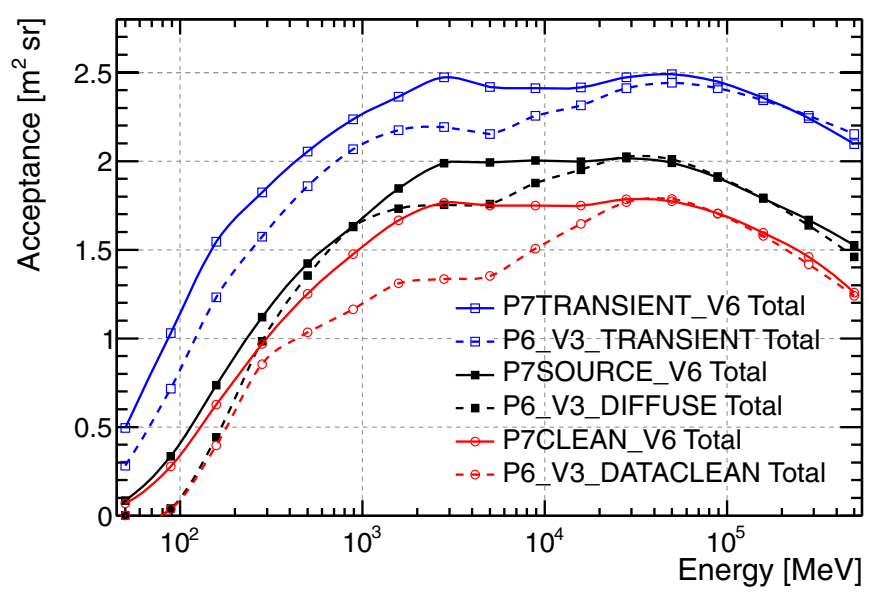

Figure 54. Comparison of the acceptances for the Pass 7 and the Pass 6 standard $\gamma$-ray classes.

(A color version of this figure is available in the online journal.)

$\sim 0.1$ at normal incidence. The transition to this measurement precision-dominated regime as predicted by the MC should occur between $\sim 3 \mathrm{GeV}$ and $\sim 20 \mathrm{GeV}$. Estimates of the limiting PSF from flight data however indicate a worse performance above $\sim 3 \mathrm{GeV}$; the PSF $68 \%$ containment levels off at almost double the calculated value (i.e., $\sim 0.16$ ). This departure is one of the few instances where the MC results significantly differ from real data (see Section 6.2). The LAT Collaboration has identified limitations in the pre-launch calibration algorithms of the CAL light asymmetry (for more details see Abdo et al. 2009a) as the primary cause of these discrepancies, and is assessing the improvement of the flight data-derived PSF at high energies for data which were reprocessed with improved calibration constants $^{84}$ (see Section 3.3.3 for details of how the CAL energy centroid is used in the event direction analysis).

\subsection{Point-spread Function from Monte Carlo Simulations}

Equivalently to what we stated in Section 1, the PSF is the likelihood to reconstruct a $\gamma$ ray with a given angular deviation $\delta v=\left|\hat{v}^{\prime}-\hat{v}\right|$. We write it as $\mathrm{P}$.

As for the effective area, events from a dedicated allGamma MC simulation that pass the selections for the event class in question are binned in true energy and incidence angle. Note again that we ignore any $\phi$ dependence of the PSF. As discussed below, in almost all cases the $\phi$ dependence of the PSF is much weaker than the $\theta$ dependence, and in Section 8.4 we show that ignoring the $\theta$ dependence induces at most a $\sim 4 \% \mathrm{rms}$ variation of the flux. To allow for some additional smoothing of the variations of the parameters of the PSF fitting function with energy and angle a procedure based on running averages is implemented: in each bin events belonging to nearby bins (by default \pm 1 bin, where the bin sizes are 0.1 in $\cos \theta$ and 0.25 decades in energy) along the energy and angle axes are included. For every bin, we build a histogram with the angular deviations of detected $\gamma$ rays. This distribution is fitted and the fit parameters are saved. Note that, although the PSF is parameterized in the LAT reference frame, the angular deviation is the same whether expressed in celestial or LAT reference frames.

Since the PSF varies with $\theta$, it is often useful to consider the PSF averaged over the observing profile (Section 2.3) for a

\footnotetext{
84 http://fermi.gsfc.nasa.gov/ssc/data/analysis/documentation/Cicerone/ Cicerone_LAT_IRFs/IRF_PSF.html
} 

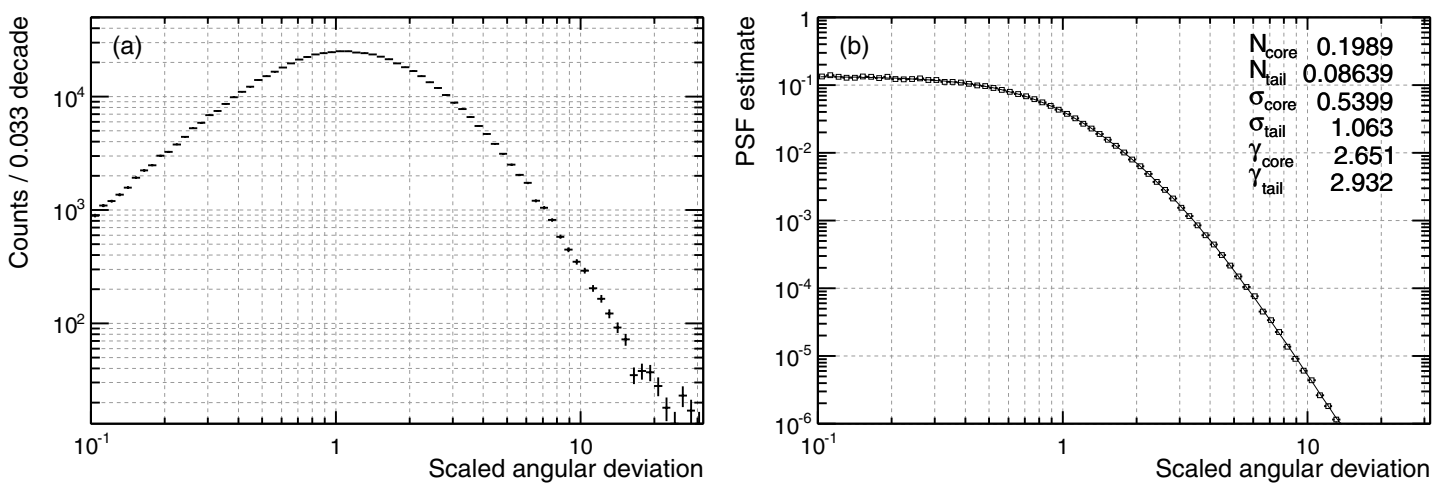

Figure 55. (a) Scaled angular deviation histogram and (b) PSF estimate in the range $E=[5.6,10] \mathrm{GeV}$ and $\theta=[26,37]^{\circ}$ for the P7SOURCE_V6 event class, front section.

Table 13

Parameters of the Angular Deviation Scaling Function $S_{P}$ for the PSF Parameterization

\begin{tabular}{lccc}
\hline \hline Conversion Type & $\begin{array}{c}c_{0} \\
\left({ }^{\circ}\right)\end{array}$ & $\begin{array}{c}c_{1} \\
\left({ }^{\circ}\right)\end{array}$ & $\beta$ \\
\hline Front & 3.32 & 0.022 & 0.80 \\
Back & 5.50 & 0.074 & 0.80
\end{tabular}

source of interest:

$$
\bar{P}(\delta v ; E)=\frac{\int P(\delta v ; E, \theta) A_{\mathrm{eff}}(E, \theta) t_{\mathrm{obs}}(\theta) d \Omega}{\int A_{\mathrm{eff}}(E, \theta) t_{\mathrm{obs}}(\hat{\theta}) d \Omega} .
$$

\subsubsection{Point-spread Function: Scaling and Fitting}

In our parameterized description of the PSF most of the energy dependence is factored into a scaling term:

$$
S_{P}(E)=\sqrt{\left[c_{0} \cdot\left(\frac{E}{100 \mathrm{MeV}}\right)^{-\beta}\right]^{2}+c_{1}^{2}}
$$

Despite a careful investigation, we did not find a simple satisfactory description of the $\theta$ dependence to be incorporated in the scaling function.

When building our MC-based PSF, we use a set of scaling function parameters based on pre-launch simulations and confirmed with analysis from beam tests with the Calibration Unit (see Section 7.3.1 for more details). The values of these parameters are shown in Table 13. Note that $S_{P}(\mathrm{E})$ has same functional form as for $C_{68}$ in Section 3.3.3, however we have updated the parameters slightly based on the scaling observed in our allGamma sample.

We define the scaled angular deviation $x$ as

$$
x=\frac{\delta v}{S_{P}(E)} .
$$

An example of scaled deviation is shown in Figure 55. The effect of the scaling is to make the profile almost independent of energy, in that the maximum is always close to $x=1$ for all energy bins while the PSF $68 \%$ containment varies by almost two orders of magnitude from $100 \mathrm{MeV}$ to $100 \mathrm{GeV}$.

Before the fit is performed, each scaled deviation histogram is converted into a probability density with respect to solid angle. The result is illustrated in Figure 55.

Note that, although the scaling removes most of the energy dependence, the simulation indicates significant variation of the PSF with $\theta$. At larger incidence angle the tracks must cross more material in each TKR plane. At energies below $\sim 1 \mathrm{GeV}$, this degrades the PSF owing to the increased multiple scattering, while at higher energies (above $\sim 1 \mathrm{GeV}$ ), the additional complication of hard scattering processes in the TKR and additional hits in the TKR from the nascent electromagnetic shower complicate the track-finding and degrade the PSF. Figure 56 shows how the scaled containment radii evolve with energy and incidence angle. To test the $\phi$ dependence of the PSF we have also measured the containment radii independently for events with $\xi>0.33$ and $\xi<0.33$, where $\xi$ is the folded azimuthal angle defined by Equation (15). The $68 \%$ and $95 \%$ containment radii for the two $\xi$ ranges differ by $<5 \%$ for all energies and angles except at high energies $(>10 \mathrm{GeV})$ and low incidence angles $(\cos \theta>0.7)$ for back-converting events, and at even higher energies $(>100 \mathrm{GeV})$ and large incidence angles $(\cos \theta<0.7)$ for front-converting events. Even then the maximum difference in the $68 \%$ containment radius for events is only $10 \%$ at $10 \mathrm{GeV}$ and $25 \%$ at $100 \mathrm{GeV}$. In summary, the variations of the containment radii with $\phi$ are many times smaller than the corresponding variations with $\theta$ for all but the highest energies $(\gtrsim 100 \mathrm{GeV})$.

The base function for the PSF is the same as used by the XMM-Newton mission (Kirsch et al. 2004; Read et al. 2011):

$$
K(x, \sigma, \gamma)=\frac{1}{2 \pi \sigma^{2}}\left(1-\frac{1}{\gamma}\right) \cdot\left[1+\frac{1}{2 \gamma} \cdot \frac{x^{2}}{\sigma^{2}}\right]^{-\gamma},
$$

which Kirsch et al. (2004) refer to as a King function (King 1962), and is isomorphic to the well-studied Student's $t$-distribution (Student 1908). Note that this function is defined so as to satisfy the normalization condition:

$$
\int_{0}^{\infty} K(x, \sigma, \gamma) 2 \pi x d x=1
$$

the extra $2 \pi x$ comes from the integral over the solid angle $d \Omega=\sin (x) d x d \phi \sim 2 \pi x d x$. However, at very low energies the PSF widens to the point that the small angle approximation fails by more than a few percent and $K(x) \sin (x)$ must be normalized numerically.

To allow for more accurate descriptions of the tails of the distributions, we use the sum of two King functions to represent the dependence of the PSF on scaled deviation for a given incidence angle and energy:

$P(x)=f_{\text {core }} K\left(x, \sigma_{\text {core }}, \gamma_{\text {core }}\right)+\left(1-f_{\text {core }}\right) K\left(x, \sigma_{\text {tail }}, \gamma_{\text {tail }}\right)$. 

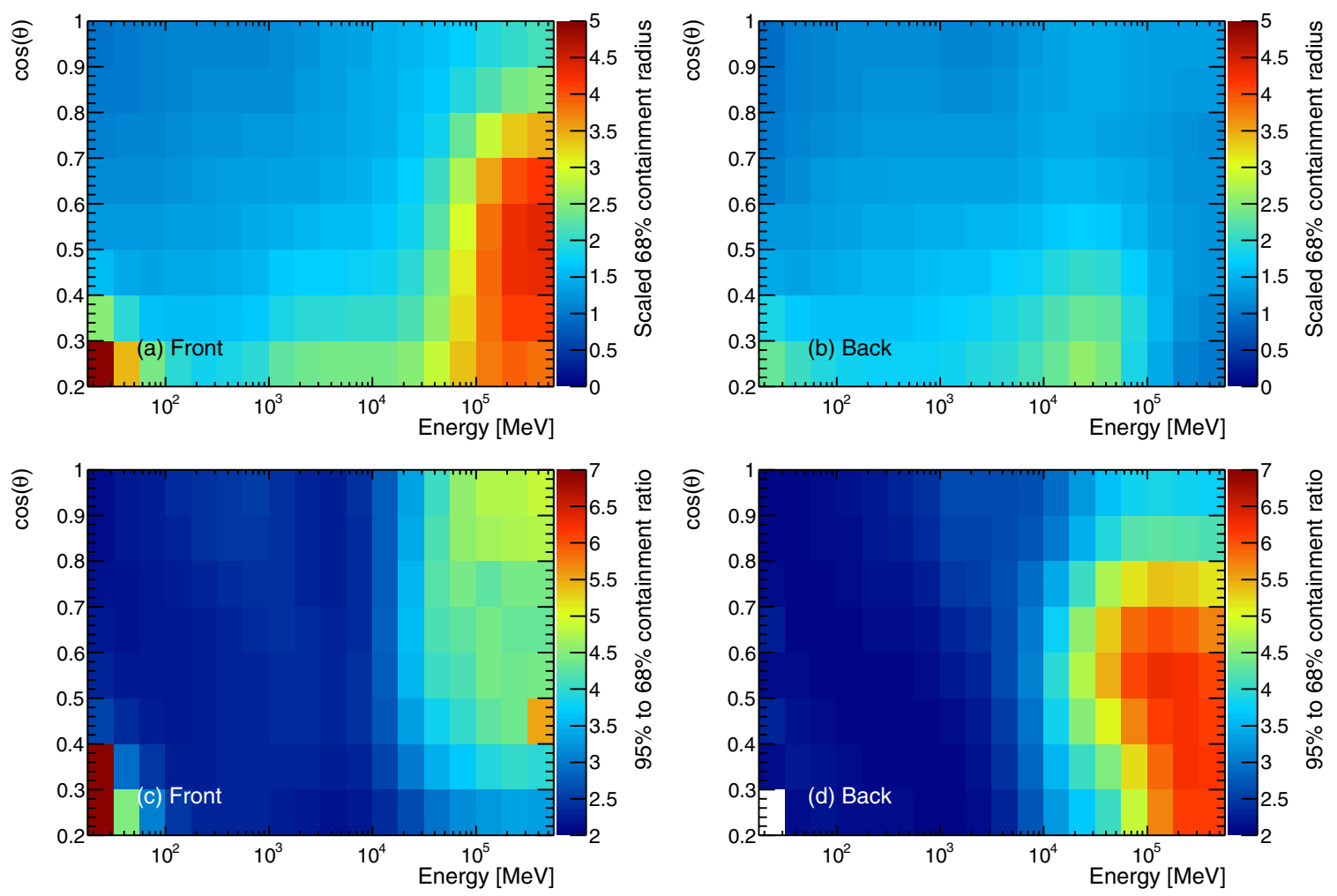

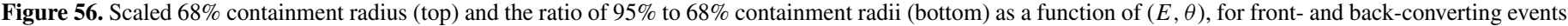
in the P7SOURCE_V6 IRFs.

(A color version of this figure is available in the online journal.)

The $\sigma$ and $\gamma$ values are stored in tables of PSF parameters as SCORE, STAIL, GCORE and GTAIL respectively. Because of the arbitrary normalization used in fitting the PSF function, $f_{\text {core }}$ must be extracted from the NTAIL table parameter, in conjunction with SCORE and STAIL:

$$
f_{\text {core }}=\frac{1}{1+\mathrm{NTAIL} \cdot \mathrm{STAIL}^{2} / \mathrm{SCORE}^{2}} .
$$

The fitting function has been revised several times since the development of the first preliminary response functions. The version described here is the one currently being used and is different from e.g., that used for P6_V3 IRFs. A description of the fit functions used in the past is given in Section 6.1.2.

\subsubsection{Legacy Point-spread Function Parameterization}

The first set of publicly released IRFs, P6_V3, used a slightly different PSF parameterization. Specifically, it allowed for only one $\sigma$ parameter and fixed the relative normalization of the two King functions by constraining the two to contribute equally at $x_{b}=2 \sqrt{5} \sigma$. So for P6_V3 we used

$$
P(x)=f_{\text {core }} K\left(x, \sigma, \gamma_{\text {core }}\right)+\left(1-f_{\text {core }}\right) K\left(x, \sigma, \gamma_{\text {tail }}\right),
$$

with

$$
f_{\text {core }}=\frac{1}{1+K\left(x_{b}, \sigma, \gamma_{\text {core }}\right) / K\left(x_{b}, \sigma, \gamma_{\text {tail }}\right)} .
$$

\subsection{Point-spread Function from On-orbit Data}

During the first year of the mission, we observed that for energies greater than a few $\mathrm{GeV}$ the distributions of $\gamma$ rays around isolated point sources were systematically wider than the expectations based on the PSF estimated from the MC simulations. We observed the same discrepancies for pulsars and blazars. In order to obtain a more accurate description of the core of the PSF for sources that are observed at a typical range of incidence angles, starting from the then-current Pass 6 IRFs, we derived the PSF directly from flight data, by means of a stacking analysis of selected point sources. The details of this analysis are described in Ackermann et al. (2012e). Here we summarize the procedure, the associated uncertainties, and the impact on high-level source analysis.

\subsubsection{Angular Containment from Pulsars}

In the 1-10 GeV energy range bright pulsars are excellent sources for evaluating the in-flight PSF: not only they are among the brightest $\gamma$-ray sources, providing abundant statistics, the pulsed emission is very stable and the angular distributions of $\gamma$ rays around the true positions can be estimated readily by phase selecting $\gamma$ rays. The Vela pulsar is the brightest pulsar and an analysis with adequate statistics can be based on Vela alone. In the remainder of this section, we describe the procedure for Vela only; the extension to an arbitrary number of pulsars is straightforward (see Ackermann et al. 2012e).

We use the P7SOURCE Vela calibration sample, which we divided into front- and back-converting subsamples, and bin the data in 4 energy bins per decade. We then calculate the pulsar phase for each $\gamma$ ray. As mentioned in Section 3.6.1, we define $[0.12,0.17] \cup[0.52,0.57]$ as the "on" interval and $[0.8,1.0]$ as "off" (see Figure 15 for the phase histogram). Next we calculate the containment angles: the position of Vela is known with a precision that greatly exceeds the LAT angular resolution, which can be assumed as the true source position. We make histograms of the angular deviations from Vela for 
the on-peak and off-pulse intervals and normalize them for the relative phase ranges. To estimate the PSF from flight data, we measure the containment radii from the difference between the histograms.

\subsubsection{Angular Containment from Active Galactic Nuclei}

Above $\sim 10 \mathrm{GeV}$ spectral cutoffs of pulsars leave AGNs as the only attractive sources for studying the PSF. In Ackermann et al. (2012e), we address the potential contribution from pair halos around AGN and conclude that we see no indication that this phenomenon is the explanation for the PSF being broader than predicted. Thus, we treat AGNs as point sources. Many $\gamma-$ ray sources are considered to be only "associated" with AGNs, as opposed to "firmly identified" (see Nolan et al. 2012, for a discussion of the distinction), because of the limited angular resolution of the LAT. In the present analysis we consider only sources with high-confidence associations. As for pulsars, the positions of AGN are known with high precision from other wavelengths, and the angular distances from the true directions can be calculated. To accumulate enough statistics we stacked several sources and performed a joint analysis. We selected AGNs from among the LAT sources with the highest significances above $10 \mathrm{GeV}$ outside the Galactic plane. This energy limit is set by the source density: below a few $\mathrm{GeV}$ the LAT PSF is broad enough that nearby sources frequently overlap.

A significant difference with respect to the pulsar analysis is the necessity of modeling the background in evaluating the distribution of angular deviations. We assume that after the stacking of the sky regions far from the Galactic plane the background count distribution can be assumed to be isotropic. At each energy the background is modeled as a flat distribution normalized by the amplitude in an annulus centered on the stacked data set. The inner radius of this annulus was chosen to be significantly larger than the region containing $\gamma$ rays from the stacked AGN sample. The uncertainty of the containment radius in each energy bin was set to the rms of a large sample of MC realizations for the signal and background distributions.

\subsubsection{Point-spread Function Fitting}

We have developed a procedure (described in detail in Ackermann et al. 2012e), to fit our PSF model to the measured containment radii for different energy ranges. Given the statistical limitations we use a single King function, Equation (36). For the same reason we do not measure the dependence of the PSF on the incidence angle, i.e., we calculate an acceptanceweighted average over the incidence angle. We first fit the experimental $68 \%$ and $95 \%$ containment radii $\left(R_{68}\right.$ and $\left.R_{95}\right)$ with Equation (36). Then we extract a new scaling relation. And finally, we use the fitted (rather than the measured) $68 \%$ and $95 \%$ containment radii to obtain a new set of PSF parameters for each energy bin. By using the fitted containment radii, this procedure smooths out the statistical fluctuations across the energy bins.

The $68 \%$ and $95 \%$ angular containment radii for the flightbased P7SOURCE_V6 PSF are shown in Figure 57.

\subsection{Uncertainties of the Point-spread Function}

The uncertainty of the derived PSF was estimated by comparing the $68 \%$ and $95 \%$ PSF containment radii from a set of calibration point sources with the corresponding containment radii derived from the P7SOURCE_V6 PSF (Ackermann et al.

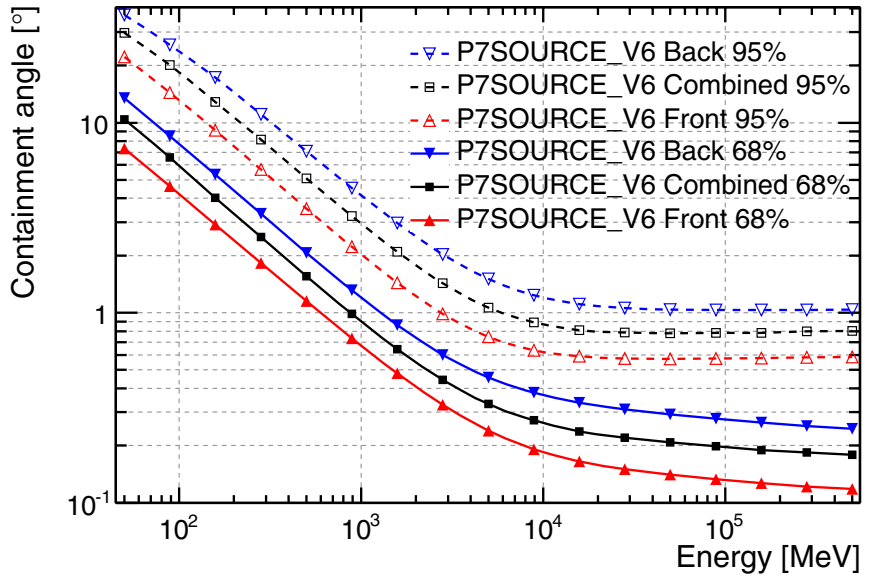

Figure 57. $68 \%$ and $95 \%$ containment angles as a function of energy for the P7SOURCE_V6 event class.

(A color version of this figure is available in the online journal.)

2012e). The $68 \%$ and $95 \%$ containment radii measure of the accuracy representation of the PSF in the core and tail, respectively.

The analysis was performed as a function of energy with four energy bins per decade. To determine the accuracy of the PSF fit as a function of incidence angle, subsamples were also studied in which $\gamma$ rays were additionally split into three bins of $\cos \theta$ $([0.2,0.5),[0.5,0.75)$, and $[0.75,1.0])$.

Figure 58 shows the $68 \%$ and $95 \%$ containment radii as a function of energy for front- and back-converting $\gamma$ rays averaged over incidence angle. The smooth lines show the model predictions for the MC (P7SOURCE_V6MC) and in-flight (P7SOURCE_V6) IRFs. At energies below $3 \mathrm{GeV}$ the containment radii match the MC PSF with fractional residuals no larger than $10 \%$. Above $3 \mathrm{GeV}$ the MC PSF begins to systematically underestimate the $68 \%$ containment radius by as much as $50 \%$ for both front- and back-converting $\gamma$ rays. As shown in Figure 58, the P7SOURCE_V6 PSF reproduces the flattening of the energy dependence of the PSF containment at high energies. However, owing to the limitations of using a single King function to parameterize the PSF, this model overpredicts the PSF tails as represented by the $95 \%$ containment radii.

At large incidence angles $(\cos \theta \in[0.2,0.5])$ the LAT PSF broadens by approximately a factor of 1.5. Due to the sky survey observing mode of Fermi and the decreased effective area at large incidence angles the fit of the in-flight PSF is dominated by $\gamma$ rays at smaller incidence angles $(\cos \theta \in[0.5,1.0])$. The variation of the PSF with incidence angle is most relevant for the analysis of transient phenomena in which the timescale of interest is comparable to or shorter than the orbital period of Fermi, such as GRBs and short-period time-series analyses. As shown in Figure 59, the agreement of in-flight and MC PSF models with the data appears worse at large incidence angles $(\cos \theta \in[0.2,0.5])$ although the limited statistics limit a rigorous comparison. The in-flight PSF model, which does not incorporate $\theta$ dependence, significantly underpredicts the width of the $68 \%$ containment radius for both front- and backconverting $\gamma$ rays. The effect of these discrepancies on highlevel analysis will be considered in Section 6.5.

\subsection{The "Fisheye" Effect}

For sources observed only in a narrow range of incidence angle, particularly near the edges of the LAT FoV, we must consider an additional complication: particles that scatter toward 

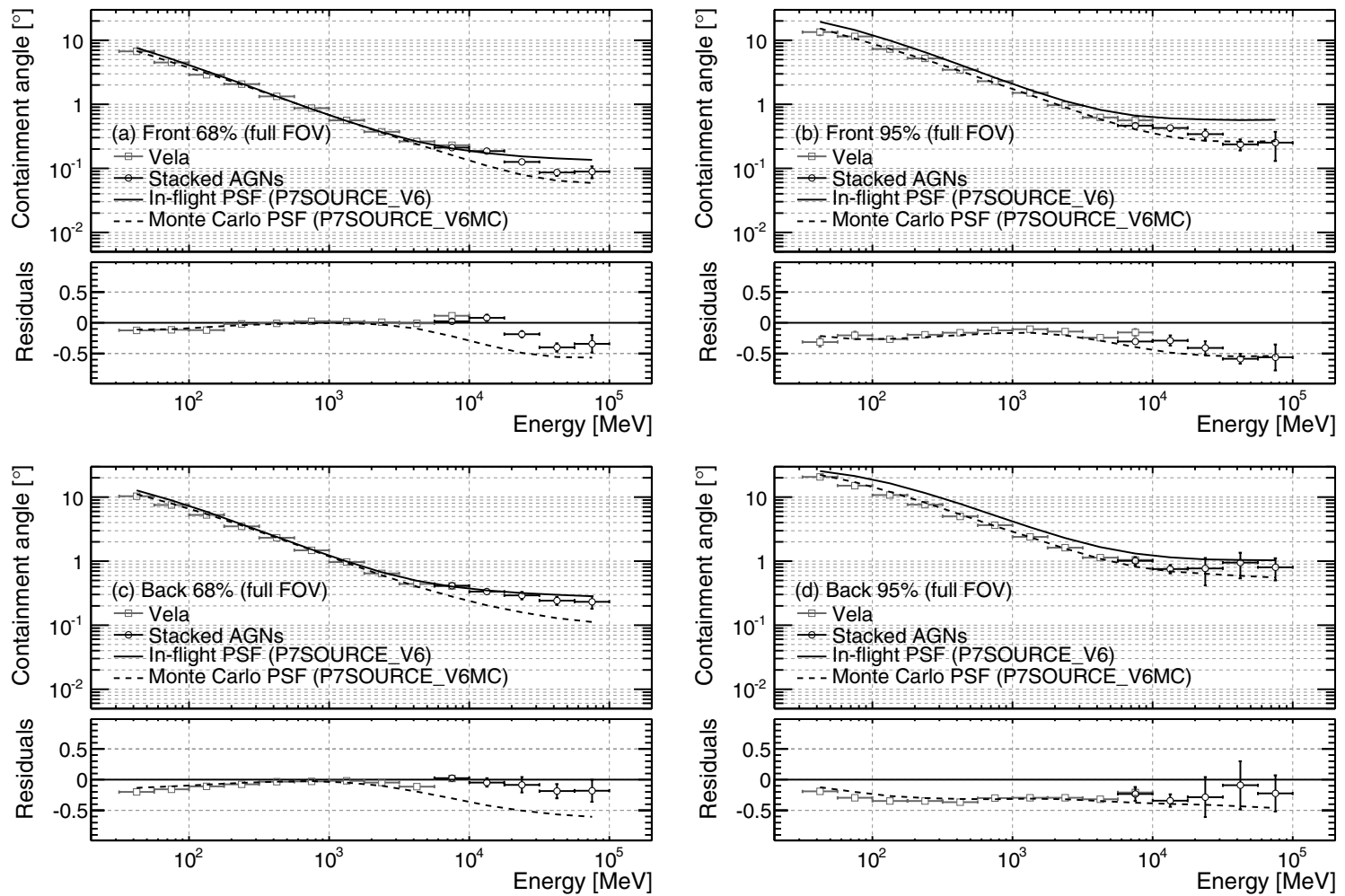

Figure 58. 68\% and 95\% containment radii for $\gamma$ rays averaged over all incidence angles ( $\cos \theta \in[0.2,1.0]$ ) as a function of energy for front (top) and back (bottom). Data points with error bars show the measured containment radii derived from the Vela and AGN PSF calibration data sets. Solid lines show the P7SOURCE_V6 model predictions in each energy bin; dashed lines show the predictions from the MC simulations (P7SOURCE_V6MC). Residual plots indicate the fractional deviation with respect to P7SOURCE_V6.
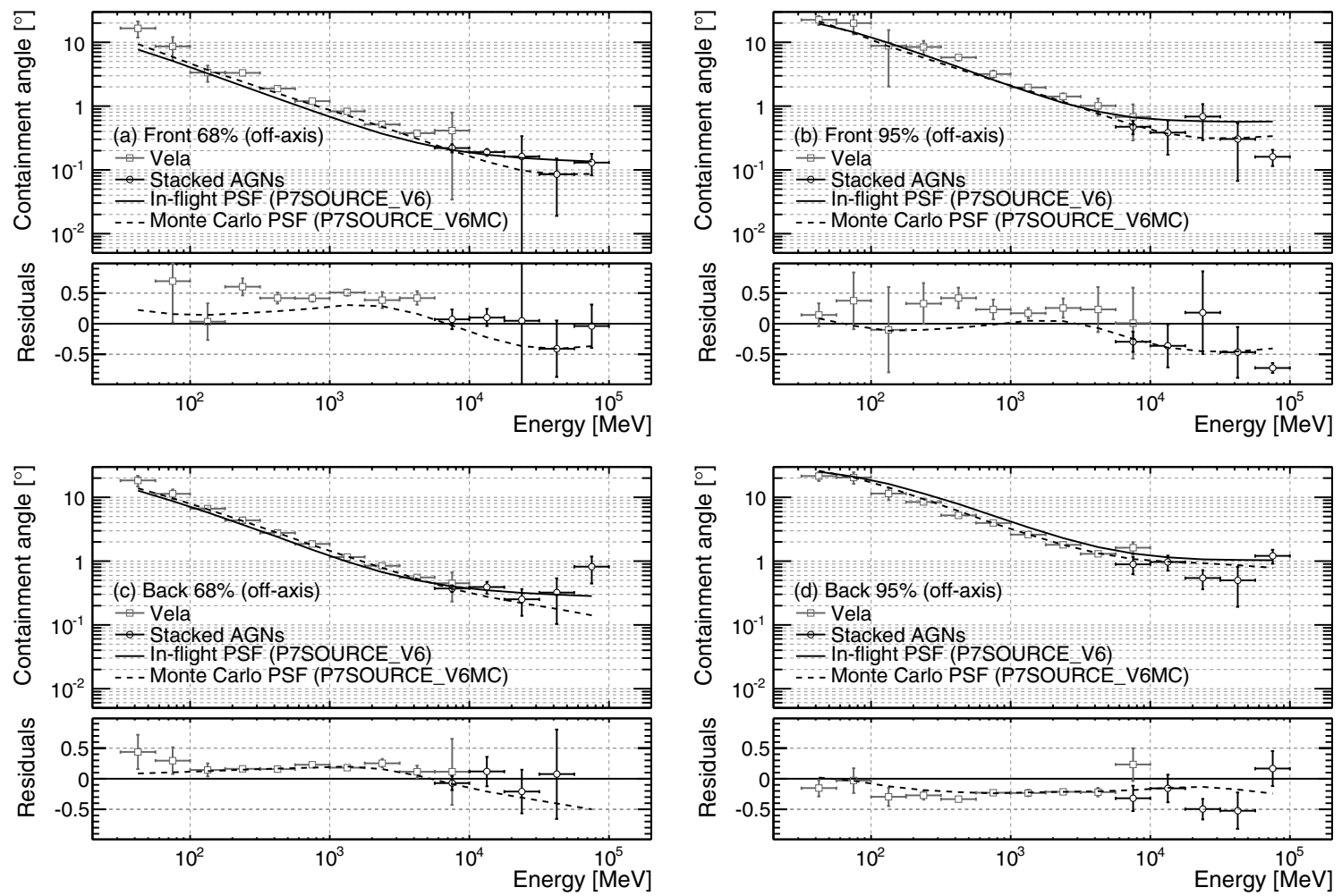

Figure 59. 68\% and 95\% containment radii for off-axis $\gamma$ rays $(\cos \theta \in[0.2,0.5])$ as a function of energy for front (top) and back (bottom). Data points with error bars show the measured containment radii derived from the Vela and AGN PSF calibration data sets. Solid lines show the P7SOURCE_V6 model predictions in each energy bin; dashed lines show the predictions from the MC simulations (P7SOURCE_V6MC). Residual plots indicate the fractional deviation with respect to P7SOURCE_V6. 

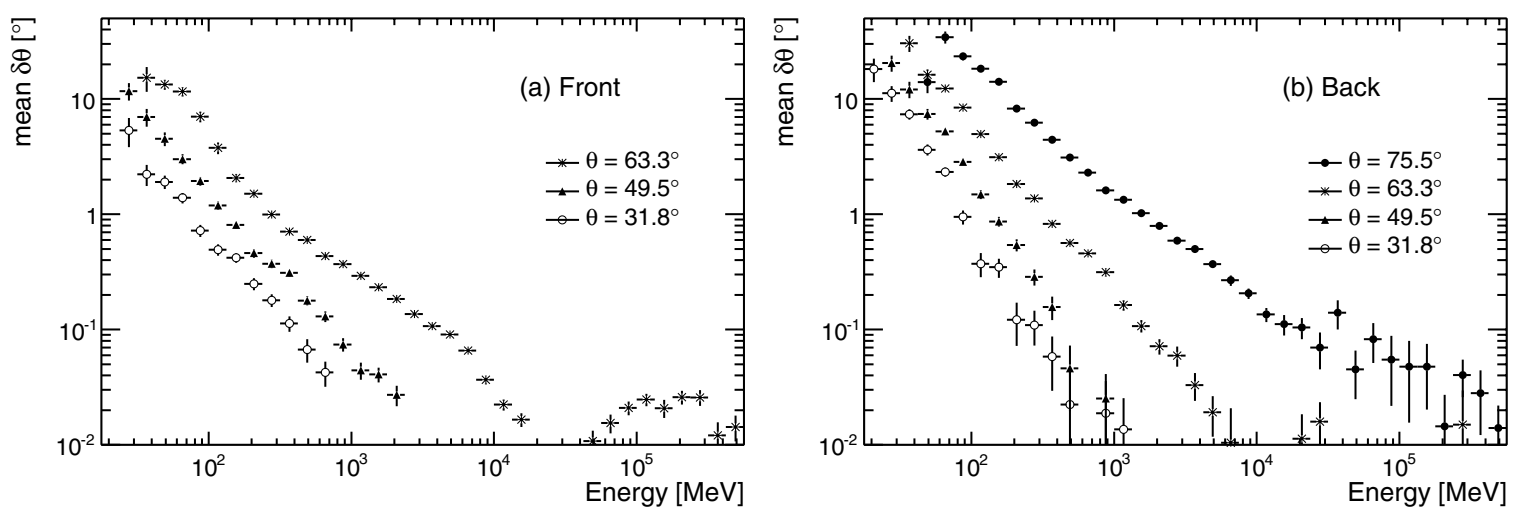

Figure 60. Mean of $\delta \theta$ for MC simulated data as a function of energy for several incidence angles for the P7SOURCE event selection for front-converting (a) and back-converting (b) events. There are not enough statistics for front-converting events at $\theta=75.5$ to extract reliable values.
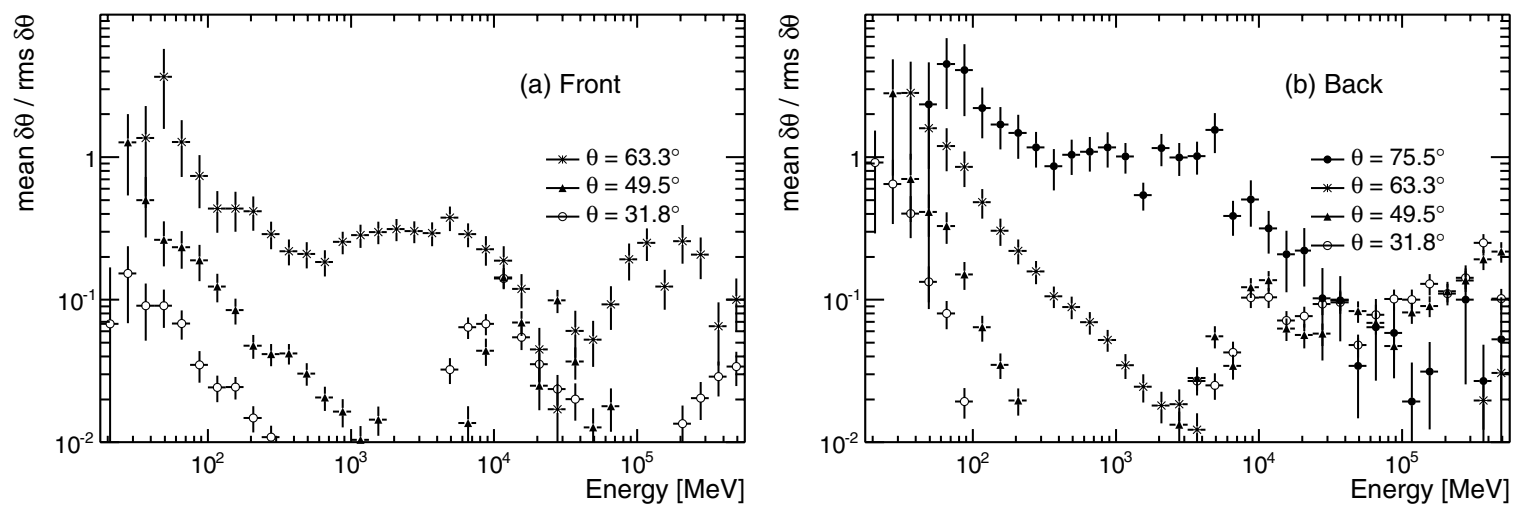

Figure 61. Ratio of the mean to the rms of $\delta \theta$ for MC simulated data as a function of energy for several incidence angles for the P7SOURCE event selection for front-converting (a) and back-converting (b) events. There are not enough statistics for front-converting events at $\theta=75.5$ to extract reliable values.

the LAT boresight are more likely to trigger the LAT and be reconstructed than particles that scatter away from the boresight. Furthermore, since the event selections do not require the separate reconstruction of both tracks from the $\gamma \rightarrow e^{+} e^{-}$ conversion and the reconstruction code will estimate an event direction using a single track if no vertex is found, for some events we base our direction estimate only on the particle that scattered more toward the LAT boresight. This effect increases as the energy decreases, since multiple scattering causes larger deviations.

Over the course of a year the position of the LAT boresight relative to any given direction in the sky is fairly uniformly distributed in azimuth; however, for shorter periods several factors can lead to large non-uniformities (see Section 2.3). Furthermore, by construction, the PSF is averaged over azimuth. Therefore, for long ( $>1$ year) integration periods the treatment of the PSF described in the previous sections is perfectly adequate and the fisheye effect simply results in a broadening of the PSF which is correctly described by the IRFs. However, for shorter periods the fisheye effect can result in systematic biases in localization, which should be accounted for. To do so we consider the polar and azimuthal components of the angular separation between the true and reconstructed $\gamma$-ray directions in the allGamma sample. Taking $\hat{v}$ as the true $\gamma$-ray direction (in the LAT frame), $\hat{v}^{\prime}$ as the reconstructed direction, and $\hat{z}$ as the LAT boresight we can define the local polar and azimuthal directions:

$$
\hat{\phi}=\frac{\hat{z} \times \hat{v}}{|\hat{z} \times \hat{v}|}, \quad \hat{\theta}=\frac{\hat{\phi} \times \hat{v}}{|\hat{\phi} \times \hat{v}|}
$$

Then we calculate the component of the misreconstruction along each:

$$
\delta \phi=\sin ^{-1}\left(\hat{\phi} \cdot\left(\hat{v}^{\prime}-\hat{v}\right)\right), \quad \delta \theta=-\sin ^{-1}\left(\hat{\theta} \cdot\left(\hat{v}^{\prime}-\hat{v}\right)\right)
$$

(the extra negative sign in the above equation is applied so that the fisheye effect represents a bias toward positive values of $\delta \theta$ ). It is also worth noting that simply considering the distributions of differences between the true and reconstructed $\theta$ is complicated by the fact that the amount of solid angle varies with $\theta$.

Figure 60 shows how the mean of $\delta \theta$ varies as a function of energy and incidence angle for the P7SOURCE event selection. Although the bias can be very large at high incidence angles and low energies, it is important to recall that (1) the PSF is quite wide in those cases and (2) there is relatively little acceptance in that region. Figure 61 shows the ratio of the mean to the rms of $\delta \theta$ for the same energies and angles, and we see that except for the furthest off-axis events and the lowest energies, the fisheye effect is a small contributor to the overall width of the PSF when considering persistent $\gamma$-ray sources.

We should recall that the P7TRANSIENT event selection does not include as tight constraints on the quality of the event reconstruction as the P7SOURCE selection. Accordingly, the fisheye effect is more pronounced for this event selection, as can be seen in Figures 62 and 63. This is of particular importance for GRBs, for which almost all of the exposure might be at a single incidence angle. For soft GRBs in the LAT, the bias for the P7TRANSIENT $\gamma$ rays near $100 \mathrm{MeV}$ that contribute the most to the localization can be up to $6^{\circ}$ at $50^{\circ}$ off-axis. Furthermore, since the orientation of the LAT boresight relative to a GRB might not change significantly during the GRB outburst, these 

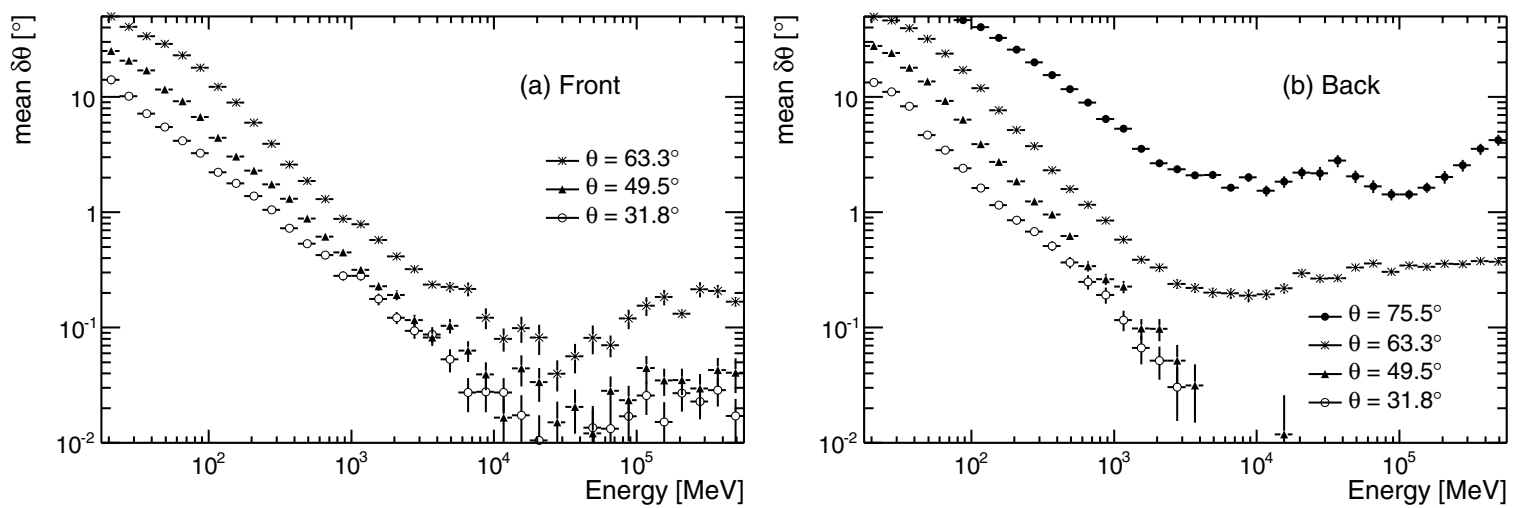

Figure 62. Mean of $\delta \theta$ for MC simulated data as a function of energy for several incidence angles for the P7TRANSIENT event selection for front-converting (a) and back-converting (b) events. There are not enough statistics for front-converting events at $\theta=75.5$ to extract reliable values.
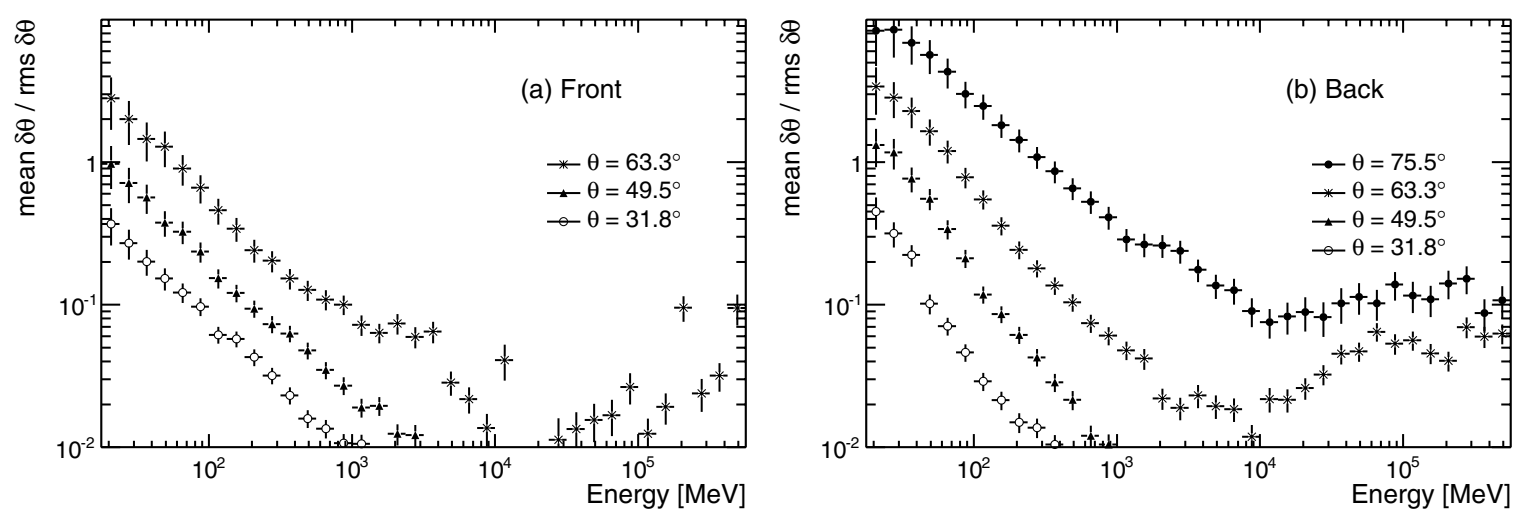

Figure 63. Ratio of the mean to the rms of $\delta \theta$ for MC simulated data as a function of energy for several incidence angles for the P7TRANSIENT event selection for front-converting (a) and back-converting (b) events. There are not enough statistics for front-converting events at $\theta=75^{\circ} 5$ to extract reliable values.

$\gamma$ rays will tend to be biased in the same direction, causing an overall bias in the localization of the GRB. Therefore, when statistics permit, more robust localizations can be obtained by using events with energies $>200 \mathrm{MeV}$, or by using P7SOURCE rather than P7TRANSIENT class events.

\subsection{Propagating Uncertainties of the Point-spread Function to High-level Science Analysis}

Uncertainties in the PSF parameterization lead to an imperfect source model in the high-level source analysis (likelihood fitting), and therefore to systematic uncertainties in source spectra (Section 6.5.1), localizations and measurements of source extensions (Section 6.5.2).

An additional source of systematic uncertainty comes from neglecting the $\theta$ dependence of the PSF in the derivation of the parameterization from flight data. In particular, this can lead to artificial variability as the PSF changes with the varying observing profile.

\subsubsection{Using Custom IRFs to Generate an Error Envelope}

We can create custom PSFs in a similar manner to that used for the effective area (see Section 5.7), though with the additional complication that we have to explore variations in the shape of the PSF as a function of energy and incidence angle. Because the dependence of the PSF on $\gamma$ is not intuitive we choose to express the bracketing functions in terms of the observable quantities $R_{68}$ and $r=R_{95} / R_{68}$ rather than in terms of $\sigma$ and $\gamma$. Specifically, in each bin of energy and incidence angle, we can
Table 14

Bracketing PSFs and the Energy-dependent Scaling Functions Used to Create Them

\begin{tabular}{lcc}
\hline \hline Name & $B_{68}(E)$ & $B_{r}(E)$ \\
\hline c_scalehi_t_nom & +1 & 0 \\
c_scalelo_t_nom & -1 & 0 \\
c_pivothit_nom & $\tanh \left(\frac{1}{k} \log \left(E / E_{0}\right)\right)$ & 0 \\
c_pivotlo_t_nom & $-\tanh \left(\frac{1}{k} \log \left(E / E_{0}\right)\right)$ & 0 \\
c_nom_t_scalehi & 0 & +1 \\
c_nom_t_scalelo & 0 & -1 \\
c_nom_t_pivothi & 0 & $\tanh \left(\frac{1}{k} \log \left(E / E_{0}\right)\right)$ \\
c_nom_t_pivotlo & 0 & $-\tanh \left(\frac{1}{k} \log \left(E / E_{0}\right)\right)$
\end{tabular}

Note. As in Section 5.7 we use $k=0.13$, which corresponds to smoothing over $\Delta E / E \sim 0.30$.

define the bracketing values $R_{68}^{\prime}$ and $r^{\prime}$ in terms of $R_{68}$ and $r$ :

$$
\begin{aligned}
R_{68}^{\prime} & =R_{68}\left(1+\epsilon_{68}(E) B_{68}(E)\right), \\
r^{\prime} & =r\left(1+\epsilon_{r}(E) B_{r}(E)\right) .
\end{aligned}
$$

We can then solve for the King function parameters $\sigma^{\prime}$ and $\gamma^{\prime}$ which would correspond to these values.

We re-analyzed both the B2 $1520+31$ and the PG $1553+113$ ROIs with each of the PSF bracketing functions listed in Table 14 using the procedure described in Section 5.7. Based on the quality of the fits described in Section 6.3, in particular the residuals on the $68 \%$ and $95 \%$ containment radii, we have 
Table 15

Fit Parameters and Integral Fluxes Obtained Using the PSF Bracketing IRFs for PG 1553+113

\begin{tabular}{lcccc}
\hline \hline Bracketing PSF & $\begin{array}{c}N_{0} \\
\left(\mathrm{MeV}^{-1} \mathrm{~cm}^{-2} \mathrm{~s}^{-1}\right)\end{array}$ & $\Gamma$ & $\begin{array}{c}F_{25} \\
\left(\mathrm{~cm}^{-2} \mathrm{~s}^{-1}\right)\end{array}$ & $\begin{array}{c}S_{25} \\
\left(\mathrm{MeV} \mathrm{cm}^{-2} \mathrm{~s}^{-1}\right)\end{array}$ \\
\hline Nominal & $2.54 \times 10^{-12}$ & 1.68 & $6.91 \times 10^{-8}$ & $1.19 \times 10^{-4}$ \\
c_nom_t_scalelo & $2.48 \times 10^{-12}$ & 1.70 & $6.94 \times 10^{-8}$ & $1.13 \times 10^{-4}$ \\
c_nom_t_scalehi & $2.54 \times 10^{-12}$ & 1.66 & $6.68 \times 10^{-8}$ & $1.22 \times 10^{-4}$ \\
c_nom_t_pivotlo & $2.46 \times 10^{-12}$ & 1.68 & $6.67 \times 10^{-8}$ & $1.15 \times 10^{-4}$ \\
c_nom_t_pivothi & $2.58 \times 10^{-12}$ & 1.68 & $6.99 \times 10^{-8}$ & $1.21 \times 10^{-4}$ \\
c_scalelo_t_nom & $2.46 \times 10^{-12}$ & 1.67 & $6.53 \times 10^{-8}$ & $1.18 \times 10^{-4}$ \\
c_scalehi_t_nom & $2.63 \times 10^{-12}$ & 1.70 & $7.32 \times 10^{-8}$ & $1.21 \times 10^{-4}$ \\
c_pivotlo_t_nom & $2.60 \times 10^{-12}$ & 1.70 & $7.30 \times 10^{-8}$ & $1.18 \times 10^{-4}$ \\
c_pivothi_t_nom & $2.49 \times 10^{-12}$ & 1.66 & $6.50 \times 10^{-8}$ & $1.21 \times 10^{-4}$ \\
\hline
\end{tabular}

Note. The quoted precision is roughly equivalent to the fit uncertainties and the pivot energy is $E_{0}=2240 \mathrm{MeV}$ for this source.

Table 16

Fit Parameters and Integral Fluxes Obtained Using the PSF Bracketing IRFs for B2 1520+31

\begin{tabular}{lccccc}
\hline \hline Bracketing PSF & $\begin{array}{c}N_{0} \\
\left(\mathrm{MeV}^{-1} \mathrm{~cm}^{-2} \mathrm{~s}^{-1}\right)\end{array}$ & $\alpha$ & $\beta$ & $\begin{array}{c}F_{25} \\
\left(\mathrm{~cm}^{-2} \mathrm{~s}^{-1}\right)\end{array}$ & $\begin{array}{c}S_{25} \\
\left(\mathrm{MeV} \mathrm{cm}^{-2} \mathrm{~s}^{-1}\right)\end{array}$ \\
\hline Nominal & $5.23 \times 10^{-10}$ & 2.24 & 0.08 & $4.09 \times 10^{-7}$ & $1.33 \times 10^{-4}$ \\
c_nom_t_scalelo & $5.34 \times 10^{-10}$ & 2.31 & 0.06 & $4.28 \times 10^{-7}$ & $1.33 \times 10^{-4}$ \\
c_nom_t_scalehi & $5.02 \times 10^{-10}$ & 2.19 & 0.09 & $3.85 \times 10^{-7}$ & $1.30 \times 10^{-4}$ \\
c_nom_t_pivotlo & $5.00 \times 10^{-10}$ & 2.22 & 0.09 & $3.86 \times 10^{-7}$ & $1.26 \times 10^{-4}$ \\
c_nom_t_pivothi & $5.43 \times 10^{-10}$ & 2.29 & 0.06 & $4.34 \times 10^{-7}$ & $1.38 \times 10^{-4}$ \\
c_scalelo_t_nom & $4.87 \times 10^{-10}$ & 2.21 & 0.08 & $3.77 \times 10^{-7}$ & $1.26 \times 10^{-4}$ \\
c_scalehi_t_nom & $5.57 \times 10^{-10}$ & 2.27 & 0.07 & $4.40 \times 10^{-7}$ & $1.40 \times 10^{-4}$ \\
c_pivotlo_t_nom & $5.27 \times 10^{-10}$ & 2.31 & 0.06 & $4.26 \times 10^{-7}$ & $1.34 \times 10^{-4}$ \\
c_pivothi_t_nom & $5.16 \times 10^{-10}$ & 2.17 & 0.10 & $3.91 \times 10^{-7}$ & $1.32 \times 10^{-4}$ \\
\hline
\end{tabular}

Note. The quoted precision is roughly equivalent to the fit uncertainties and the pivot energy is $E_{0}=281 \mathrm{MeV}$ for this source.

assigned

$$
\begin{aligned}
\epsilon_{68}(E) & =10 \%, \\
\epsilon_{r}(E) & =50 \% .
\end{aligned}
$$

Tables 15 and 16 show the fit results for PG $1553+113$ and B2 $1520+31$ as well as the integral counts and energy fluxes between $100 \mathrm{MeV}$ and $100 \mathrm{GeV}$ obtained using these PSF bracketing functions. The ranges of the fit values indicate propagated uncertainties from the uncertainty in the PSF (Table 17).

The greater influence of the uncertainty of the PSF on the flux and spectral measurements for the softer source (B2 1520+31) comes about because at lower energies the wider PSF makes resolving sources more difficult and results in greater correlation with the Galactic and isotropic diffuse components in the likelihood fit, which varied by up to $\pm 4 \%$ and $\pm 5 \%$, respectively.

\subsubsection{Effects on Source Extension}

For sufficiently long exposures, the LAT can spatially resolve a number of $\gamma$-ray sources. In the 2FGL catalog, 12 spatially extended LAT sources were identified (Nolan et al. 2012) using 24 months of LAT data; and several additional extended sources have been recently resolved from these data by Lande et al. (2012) using special techniques for modeling the spatial extension. Understanding the possible spatial extension of LAT sources is important for identifying multiwavelength counterparts, and using a source model with the correct spatial extent produces more accurate spectral fits and avoids biases in the model parameters.
Table 17

\begin{tabular}{|c|c|c|}
\hline Parameter & 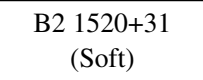 & $\begin{array}{c}\text { PG } 1553+113 \\
\text { (Hard) }\end{array}$ \\
\hline$\delta N_{0} / N_{0}$ & $+6.6 \%-6.8 \%$ & $+3.5 \%-3.4 \%$ \\
\hline$\delta \Gamma(\delta \alpha)$ & $+0.07-0.07$ & $+0.02-0.02$ \\
\hline$\delta \beta$ & $+0.02-0.02$ & $\ldots$ \\
\hline$\delta F_{25} / F_{25}$ & $+7.7 \% \quad-7.7 \%$ & $+6.0 \%-5.9 \%$ \\
\hline$\delta S_{25} / S_{25}$ & $+5.4 \% \quad-5.5 \%$ & $+2.4 \%-4.9 \%$ \\
\hline
\end{tabular}

Systematic Variations Arising from Uncertainties in the PSF

Notes. For the spectral index ( $\Gamma$ or $\alpha$ ) and spectral curvature $(\beta)$, we give the absolute variation with respect to the nominal value (e.g., $\delta \Gamma$ ). For the flux prefactor and the integral fluxes, we give the relative variations with respect to the nominal value (e.g., $\delta N_{0} / N_{0}$ ).

In addition to using a correct spatial model, the accuracy of the PSF can also affect the analyses of extended sources; and using an incorrect PSF will result in biases both in the fitted model parameters and in the significance of any spatial extension that is found. For example, flight data indicate that the MC-based PSF in the P7SOURCE_V6MC IRFs is too narrow at energies $>3 \mathrm{GeV}$ (Section 6.2). Fitting the extension of SNR IC 443 (Abdo et al. 2010g) with a uniform disk for data from the first two years of observations and using the flight-determined PSF in P7SOURCE_V6, we find a best fit disk radius of IC 443 of $\sigma=0.35 \pm 0.01$ (Lande et al. 2012). By contrast, fitting these same data, but using the MC-based PSF in P7SOURCE_V6MC, we find a best fit radius of $\sigma=0.39$. This corresponds to a $\sim 10 \%$ systematic bias in the measurement of the extension of IC 443. 

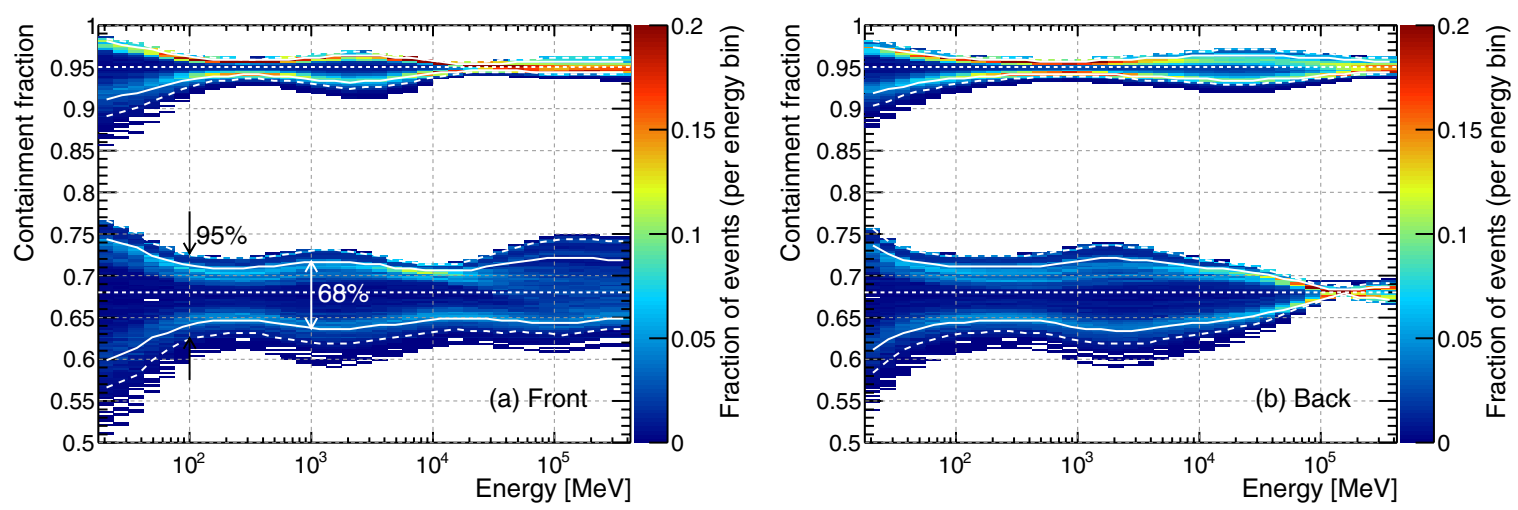

Figure 64. Fraction of the PSF contained at the mission-averaged 68\% and 95\% containment radii in the direction of the Vela pulsar for the P7SOURCE_V6MC IRFs as a function of energy for front-converting (a) and back-converting (b) $\gamma$ rays for each of the $140012 \mathrm{hr}$ time intervals.

(A color version of this figure is available in the online journal.)

Since IC 443 is a fairly hard source, with photon index $\Gamma=2.2$, the bias found for softer sources would be somewhat smaller.

Use of an imperfect model of the PSF can also affect the assessment of the statistical significance of the extension of the source. We determine the significance of a measured source extension using the test statistic

$$
\mathrm{TS}_{\mathrm{ext}}=2 \log \left(L_{\mathrm{ext}} / L_{\mathrm{pt}}\right) .
$$

This is twice the difference in log-likelihood found when fitting the source assuming it is spatially extended versus assuming it is a point source. The use and validity of this formula is described in Lande et al. (2012). For IC 443, using the P7SOURCE_V6 IRFs, we find $\mathrm{TS}_{\mathrm{ext}}=640$, corresponding to a formal statistical significance of $\sim 25 \sigma$. Performing the same analysis using P7SOURCE_V6MC, we obtain $\mathrm{TS}_{\mathrm{ext}}=1300$, corresponding to $\sim 37 \sigma$.

\subsubsection{Effects on Variability}

Neglecting the dependence of the PSF on incidence angle (Section 6.3) may introduce time-dependent biases of the estimated flux of the source, due to the different distribution of the source position with respect to the LAT boresight in each time interval. To estimate the potential size of such biases we consider how great an effect on source fluxes we might see if we were to naively use the nominal containment radius for aperture photometry analysis (i.e., an analysis where we simply count the $\gamma$ rays within a given aperture radius). Specifically, we use the MC-based PSF to extract the containment radii averaged over the first two years of the mission $\bar{R}(E)$ in the direction of the Vela pulsar. Then we split the data into much shorter time intervals (indexed by $i$ ) and compute the fraction of $\gamma$ rays $C_{i}(E$; vela) that fall within $\bar{R}(E$; vela) for each of those shorter intervals:

$$
\begin{aligned}
C_{i}(E, \text { vela }) \\
\quad=\frac{\iint_{0}^{\bar{R}(E ; \text { vela })} A_{\text {eff }}(E, \theta ; \text { vela }) t_{\mathrm{obs}, \mathrm{i}}(\theta ; \text { vela }) \sin \theta d \theta d r}{\int A_{\mathrm{eff}}(E, \theta) t_{\mathrm{obs}, \mathrm{i}}(\theta ; \text { vela }) \sin \theta d \theta} .
\end{aligned}
$$

Here we consider $68 \%$ and $95 \%$ containment radii. Figure 64 shows how $C_{68, i}\left(E\right.$; vela) and $C_{95, i}(E$; vela) vary for each $12 \mathrm{hr}$ time interval over the first 700 days of routine science operations. If there were no $\theta$ dependence to the PSF this figure would show lines at 0.68 and 0.95 . However, we clearly see that ignoring the $\theta$ dependence will cause slight misestimates in the fraction of $\gamma$ rays falling within the mission-averaged $\bar{R}_{68}$ and $\bar{R}_{95}$. In fact, the width of the bands (i.e., the spread of that misestimation) indicate the errors in flux we would expect if we were making a flux estimate based on aperture photometry with aperture cuts at $\bar{R}$. It is worth pointing out explicitly that the $C_{95, i}(E)$ band is significantly narrower, simply because most of the $\gamma$ rays are already contained within the $\bar{R}_{95}(E)$ and the derivative of the PSF is smaller.

Understanding the effect of the PSF dependence on incidence angle will have on a full likelihood analysis is much more complicated, as it depends on the other sources in the likelihood model. However, it is reasonable to take the width of the $C_{68}(E)$ bands as indicative of the magnitude of the effect when analyzing sources in complex regions where source proximity is a issue and the width of the $C_{95}(E)$ bands as indicative when analyzing isolated sources.

\subsection{Comparison of Pass 6 and Pass 7}

As a consequence of the issues discussed in Section 5.4, Pass 7 event classes have less systematic uncertainty for $A_{\text {eff }}$ at the expense of a slightly broader PSF across the entire parameter space.

In addition, Pass 7 standard classes (P7SOURCE and cleaner) feature a PSF derived from data above $1 \mathrm{GeV}$; see Section 6.2. Below $1 \mathrm{GeV}$, where the PSF is derived from MC simulations, the PSF parameters are recalculated in order to ensure a smooth variation of the containment levels as a function of energy (Section 6.2.3).

Figure 65 compares the $68 \%$ containment angles for Pass 6 and Pass 7 classes recommended for routine analyses of $\gamma$-ray point sources. While the difference between P6_V3_DIFFUSE and P7SOURCE_V6 is due mostly to the data-derived PSF in the latter, there is also a clear difference across all energies between the P7SOURCE_V6 PSF and in-flight P6_V11_DIFFUSE PSF.

While the difference in the containment radii we described is typically within the PSF uncertainties this comparison was performed with the incidence angle dependence ignored, so the tabulated PSF is an average over the FoV weighted by the average exposure over long timescales. This may be an issue for the analysis of bright sources over short timescales, when the observation angle distribution in the LAT reference system is significantly different from the average. Future Pass 7 IRFs may address this by reintroducing the $\theta$ dependence. 

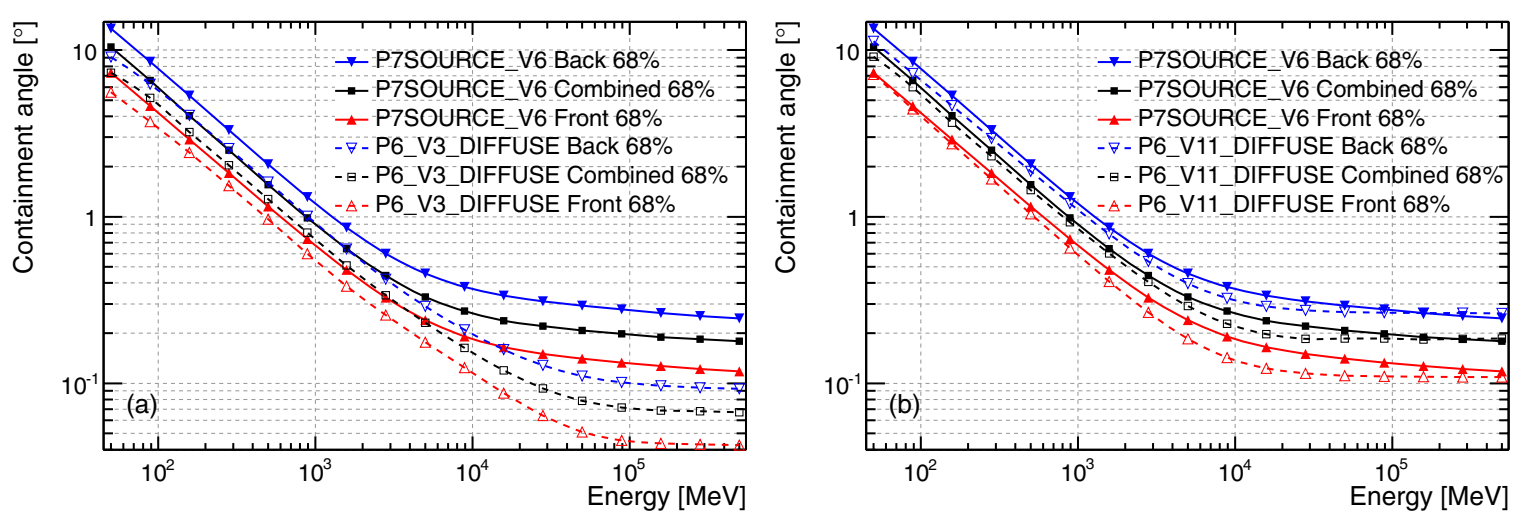

Figure 65. Comparison of the $68 \%$ containment radius of the PSF for P7SOURCE_V6 with respect to P6_V3_DIFFUSE (a) and P6_V11_DIFFUSE (b). The P6_V3_DIFFUSE is plotted for normal incidence; P6_V11_DIFFUSE and P7SOURCE_V6 do not include dependence on the incidence angle.

(A color version of this figure is available in the online journal.)

Current Pass 7 IRFs parameterize the PSF with a simplified version of Equation (38) for which the tail term is omitted as described in Section 6.2.3. The less accurate model of the PSF tails in the Pass 7 classes with on-orbit PSF (to date P7SOURCE_V6, P7CLEAN_V6, and P7ULTRACLEAN_V6) is probably not an issue for most analyses, but caution is recommended when comparing the dispersion of $\gamma$ rays around the true position of bright sources with the expected distribution derived from the IRFs.

\section{ENERGY DISPERSION}

The dispersion of measured energies around the true values (i.e., the energy dispersion, or redistribution function) is generally asymmetric, with the most prominent tail being toward lower energies. This feature, characteristic of thin electromagnetic calorimeters (such as the LAT CAL, as opposed to full-containment calorimeters) makes the energy redistribution difficult to parameterize. As a matter of fact, since most source spectra are steeply falling with energy, low-energy tails in the energy dispersion are relatively harmless, while overestimating the event energy can potentially lead to overestimating the hardness of the spectrum. In the event selections we specifically make an effort to suppress the high-energy tails, rejecting events for which we might overcompensate with the energy corrections (see Sections 3.2.1 and 3.3.2).

While the width and shape of the energy redistribution are well understood, by default the energy dispersion is not taken into account in the standard likelihood analysis, primarily due to computational limitations. Furthermore, as we will see in Section 7.4, the effect of neglecting the energy redistribution is usually small enough that it can be ignored. When that is not the case, it is easy to check the magnitude of the bias induced with dedicated simulations. If needed, the energy dispersion can be accounted for in the spectral analysis either by specifically enabling the functionality in the binned likelihood (ScienceTools version 09-26-00 or later) or by means of unfolding techniques, as we briefly describe in Section 7.4.

It is worth stressing that an in-flight validation of the energy response is much more difficult than the corresponding validations of the effective area and the PSF described in the previous section, as there is no known astrophysical calibration source that provides a spectral line at a well-defined energy that would play the role that point sources have for the PSF (see, however, the discussion in Section 7.3.3). The energy reconstruction validation will be described in Section 7.3.
Table 18

Numerical Values of the Coefficients Defining the Energy Resolution Scaling Function $S_{D}$ in Equation (48) for the Pass 6 and Pass 7 Event Classes

\begin{tabular}{ccccccc}
\hline \hline & $c_{0}$ & $c_{1}$ & $c_{2}$ & $c_{3}$ & $c_{4}$ & $c_{5}$ \\
\hline Front & 0.0210 & 0.0580 & -0.207 & -0.213 & 0.042 & 0.564 \\
Back & 0.0215 & 0.0507 & -0.220 & -0.243 & 0.065 & 0.584 \\
\hline
\end{tabular}

\subsection{Energy Dispersion and Parameterization from Monte Carlo Simulations}

As for $A_{\text {eff }}$ and PSF, in order to derive the energy dispersion events from an allGamma simulation belonging to a specified event class are binned in true energy $E$ and incidence angle $\theta$ and the distribution of measured energy $E^{\prime}$ is fitted (and the parameters tabulated). A complex functional form is necessary and attention is required to avoid fit instability and overly rapid variation of the fit parameters.

\subsubsection{Scaling}

The success of scaling the PSF distributions (see Section 6.1.1) prompted a similar approach to the histogramming and fitting of the energy deviations. The scaling function $S_{D}$ currently employed, derived by fitting the $68 \%$ containment of the measured fractional energy distribution $\left(E^{\prime}-E\right) / E$ across the entire $E-\theta$ plane, is

$$
\begin{aligned}
S_{D}(E, \theta)= & c_{0}\left(\log _{10} E\right)^{2}+c_{1}(\cos \theta)^{2}+c_{2} \log _{10} E+c_{3} \cos \theta \\
& +c_{4} \log _{10} E \cos \theta+c_{5} .
\end{aligned}
$$

Front- and back-converting events are treated separately. The parameters used since the Pass 6 analysis are listed in Table 18. These are included in the IRF files for Pass 6 and Pass 7 event classes.

The scaling function $S_{D}$ allows us to define a scaled energy deviation $x$ for which much of the energy and angular dependence is already accounted for

$$
x=\frac{\left(E^{\prime}-E\right)}{S_{D}(E, \theta) E} .
$$

This scaled value is calculated for each simulated event and histogrammed, as shown in Figure 66. Scaling the distribution achieves two main effects: the width of the core of the distribution is almost constant for all energies and incidence angles, and the distribution thus expressed is significantly simpler to parameterize than the unscaled version. 


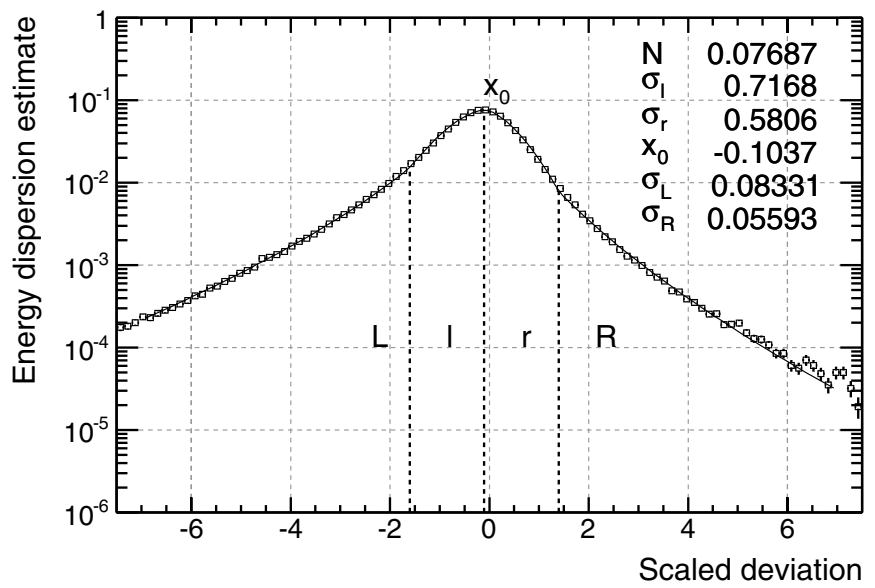

Figure 66. Histogram of the scaled energy deviation, as defined in Equation (49), fitted with the function $D(x)$ in Equation (51). The plot refers to the $(E, \theta)$ bin centered at $7.5 \mathrm{GeV}$ and $30^{\circ}$ for the front section.

Table 19

Numerical Values of the Split Point $\tilde{x}$ and of the Four Exponents $\gamma$ in Equation (51) that are Fixed When Fitting the Scaled Energy Deviations

\begin{tabular}{lllll}
\hline \hline$\tilde{x}$ & $\gamma_{L}$ & $\gamma_{l}$ & $\gamma_{r}$ & $\gamma_{R}$ \\
\hline 1.5 & 0.6 & 1.6 & 1.6 & 0.6 \\
\hline
\end{tabular}

\subsubsection{Fitting the Scaled Variable}

The scaled energy deviation, as illustrated by the example in Figure 66, has a well-defined core accompanied by elongated tails. We found we can effectively fit this structure with several piecewise functions of the form

$$
R\left(x, x_{0}, \sigma, \gamma\right)=N \exp \left(-\frac{1}{2}\left|\frac{x-x_{0}}{\sigma}\right|^{\gamma}\right)
$$

Indeed we model the energy dispersion $D(x)$ within each $(E, \theta)$ bin as four piecewise functions:

$$
D(x)= \begin{cases}N_{L} R\left(x, x_{0}, \sigma_{L}, \gamma_{L}\right) & \text { if }\left(x-x_{0}\right)<-\tilde{x} \\ N_{l} R\left(x, x_{0}, \sigma_{l}, \gamma_{l}\right) & \text { if }\left(x-x_{0}\right) \in[-\tilde{x}, 0] \\ N_{r} R\left(x, x_{0}, \sigma_{r}, \gamma_{r}\right) & \text { if }\left(x-x_{0}\right) \in[0, \tilde{x}] \\ N_{R} R\left(x, x_{0}, \sigma_{R}, \gamma_{R}\right) & \text { if }\left(x-x_{0}\right)>\tilde{x} .\end{cases}
$$

The values of the split point $\tilde{x}$ and of the four exponents $\gamma$ of the energy dispersion parameterization in Equation (51) are fixed as specified in Table 19. Moreover, the relative normalizations are set by requiring continuity at $x=x_{0}$ and $\left|x-x_{0}\right|=\tilde{x}$ and therefore the fit is effectively performed with a total of six free parameters, which are stored in the IRF FITS files: the overall normalization $N_{r}=N_{l}$ (NORM), the centroid position $x_{0}$ (BIAS), the two core scales $\sigma_{r}$ (RS1) and $\sigma_{l}$ (LS1), and the two tail scales $\sigma_{R}(\mathrm{RS} 2)$ and $\sigma_{L}(\mathrm{LS} 2)$.

\subsubsection{Energy Resolution}

The energy resolution is a figure of merit which is customarily used to summarize in a single number the information contained in the energy dispersion parameterization. As illustrated in Figure 67, we define the energy resolution as the half-width of the energy window containing $34 \%+34 \%$ (i.e., $68 \%$ ) of the energy dispersion on both sides of its most probable value, divided by the most probable value itself. We note that this

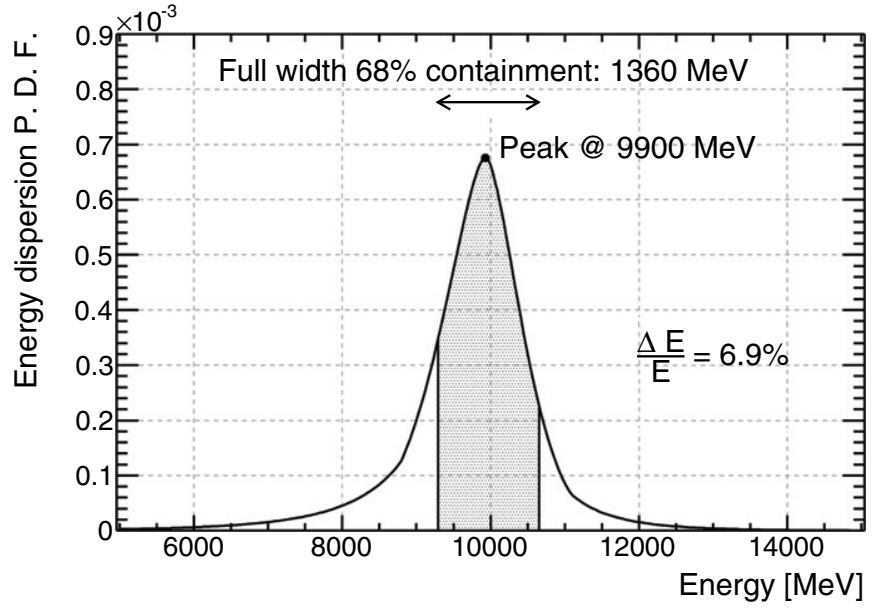

Figure 67. Energy dispersion at $10 \mathrm{GeV}$ for front-converting P7_SOURCE events $30^{\circ}$ off-axis. The most probable value of the distribution and the $68 \%$ containment window are indicated.

prescription gives slightly larger values of energy resolution than using the smallest $68 \%$ containment window.

Figures 68 and 69 show the energy resolution for P7SOURCE events as a function of energy and incidence angle. As mentioned in Section 2.1.2 the energy resolution has a broad minimum between $\sim 1$ and $\sim 10 \mathrm{GeV}$, degrading at lower energies due to the energy deposited in the TKR and at higher energies due to the leakage of the electromagnetic shower out the sides and the back of the CAL. Conversely, the energy resolution tends to improve as the incidence angle increases. This is especially true at high energy, where a longer path length in the CAL implies less shower leakage - though front-converting events more than $55^{\circ}$ off-axis tend to exit the sides of the CAL and have worse energy resolution.

\subsubsection{Correlation between Energy Dispersion and PSF}

As stated in Section 1, we factorize the IRFs, effectively assuming that the energy and direction measurements are uncorrelated. We use our allGamma MC sample to test this hypothesis. We locate each event within the cumulative PSF and measured energy distributions:

$$
\begin{aligned}
& P_{P}=\int_{0}^{\alpha} P(\alpha ; E, \hat{v}) d \alpha, \\
& P_{D}=\int_{0}^{E^{\prime}} D\left(E^{\prime} ; E, \hat{v}\right) d E^{\prime} .
\end{aligned}
$$

If the IRFs perfectly described the MC sample, both of these distributions should be flat. Furthermore, if there are no correlations between the energy measurement and the direction estimate, the two-dimensional combined distribution should also be flat. In practice this is nearly the case; the correlation coefficient between $P_{P}$ and $P_{D}$ is small $\left(\left|C_{P, D}\right|<0.1\right)$ across most of the energy and incidence angle range, as shown in Figure 70(a). However, in many bins of $(E, \theta)$, we do observe a small excess of counts with $P_{P} \sim 1$ and $P_{D} \sim 0$. Those values correspond to events that are in the tails of the PSF and have a energy estimate that are significantly lower than the true $\gamma$-ray energy. To quantify the magnitude of this effect, we considered the fraction of highly anti-correlated events $f_{a}$ with $P_{P}>0.98$ and $P_{D}<0.02$ as a function of $(E, \theta)$. In the absence of correlations, this fraction should be $f_{a} \sim 4 \times 10^{-4}$. In fact, for highly off-axis $\gamma$ 

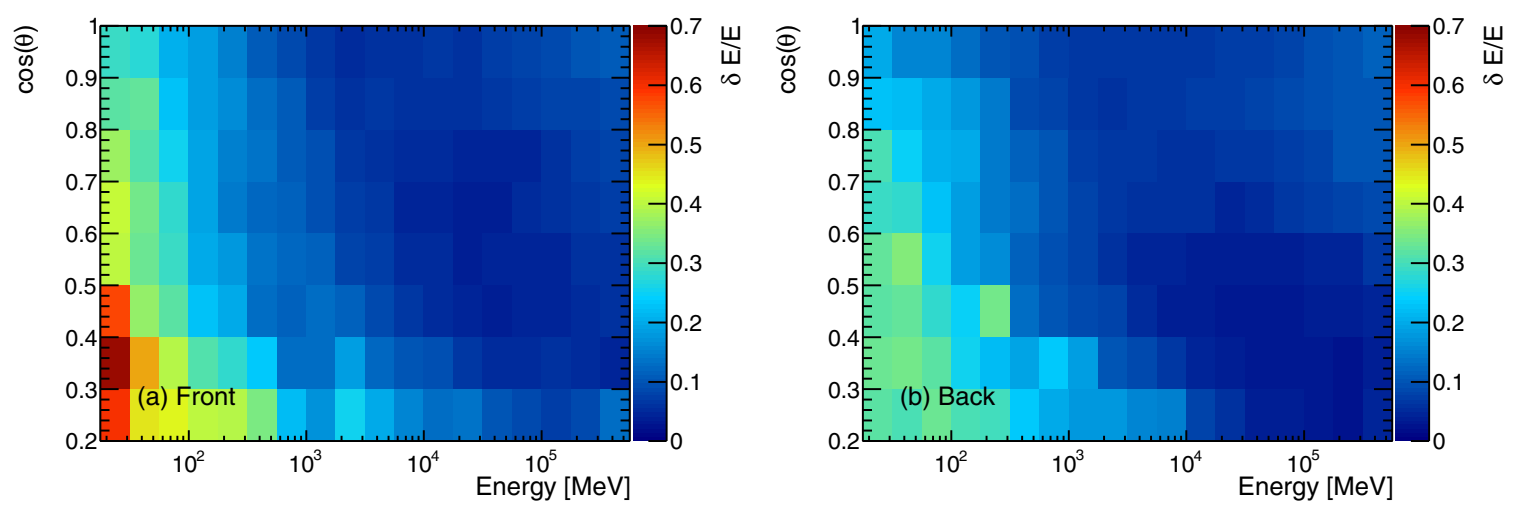

Figure 68. Energy resolution (68\% containment half-width, as described in the text) as a function of energy and incidence angle for front (a) and back (b) conversions. (A color version of this figure is available in the online journal.)
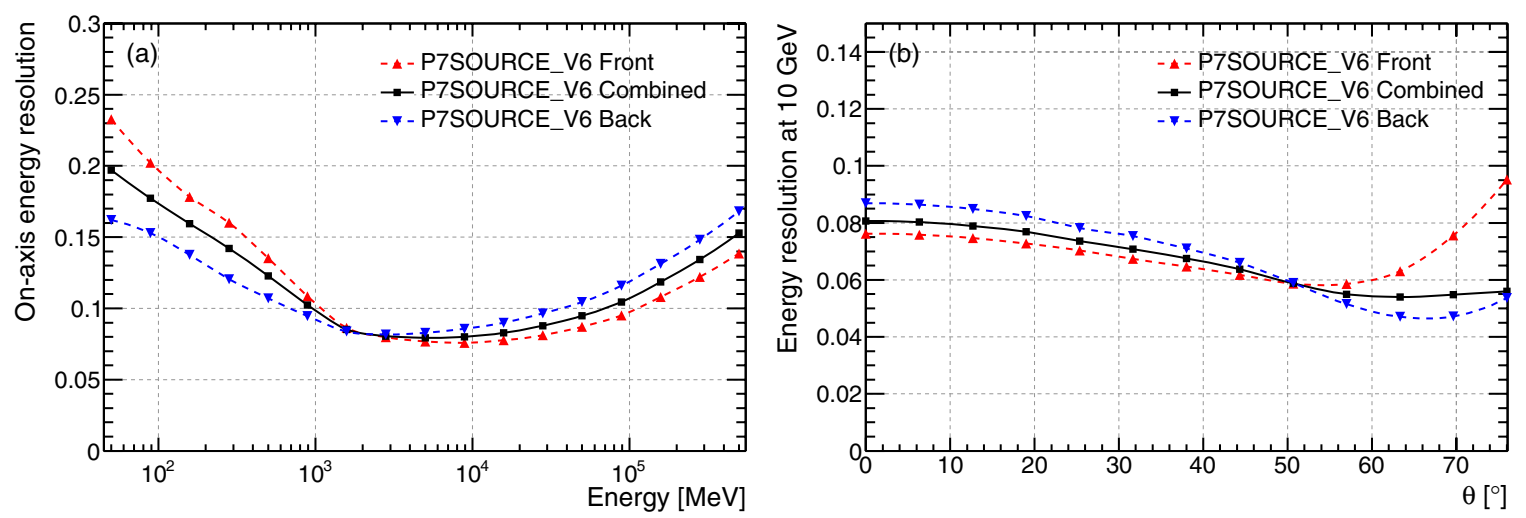

Figure 69. Energy resolution as a function of energy on-axis (a) and incidence angle at $10 \mathrm{GeV}$ (b) for the P7SOURCE_V6 event class.

(A color version of this figure is available in the online journal.)
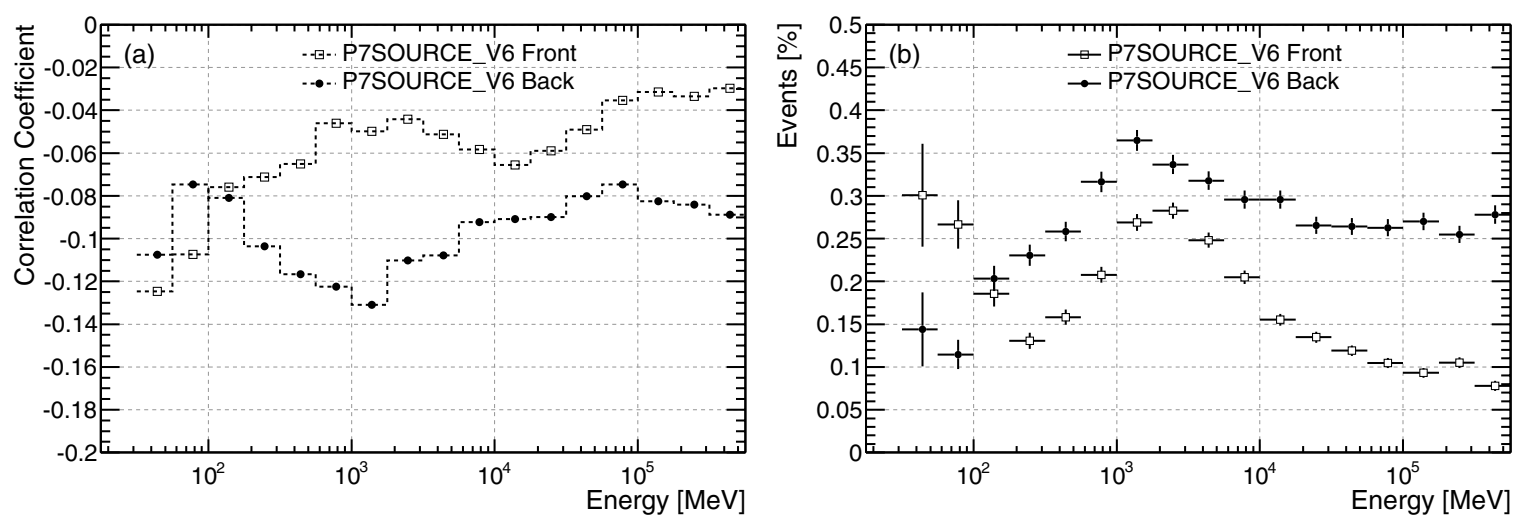

Figure 70. Correlation between the energy dispersion and the PSF in the allGamma (Section 2.5.3) sample: (a) correlation coefficient $C_{P, D}$ between $P_{P}$ and $P_{D}$ as a function of energy and (b) fraction of events with $P_{P}>0.98$ and $P_{D}<0.02$ (i.e., fraction of events which are in the tail of the PSF and also in the low side tail of the measured energy distribution).

rays in some energy ranges this fraction approaches $f_{a} \sim 0.02$; however, averaged over the FoV, it is in the $0.001-0.0035$ range for both front- and back-converting events at all energies, as shown in Figure 70(b).

Although we cannot reproduce this analysis with flight data, we have studied the correlations between the energy and direction quality estimators $\left(P_{\mathrm{E}}\right.$, see Section 3.3.2 and $P_{\text {core }}$, see Section 3.3.3) and found similarly small effects.

In summary, averaged over several orbital precession periods any biases caused by the correlation between the energy dispersion and the PSF are negligible compared to other systematic uncertainties we consider in the paper. However, it is certainly a potential contributor to instrument-induced variability (see Section 8.4).

\subsection{Spectral Effects Observed with Simulations}

As noted in Section 3.3.2, the maximum likelihood (LH) energy correction algorithm described in Section 3.2.1 (and used only in Pass 6) is by construction a binned energy estimator. We have observed that it introduces spectral artifacts corresponding to the bins used in creating the likelihood parameterization. In addition, due to the fact that the correction is not reliable above a few hundred $\mathrm{GeV}$, the method is 

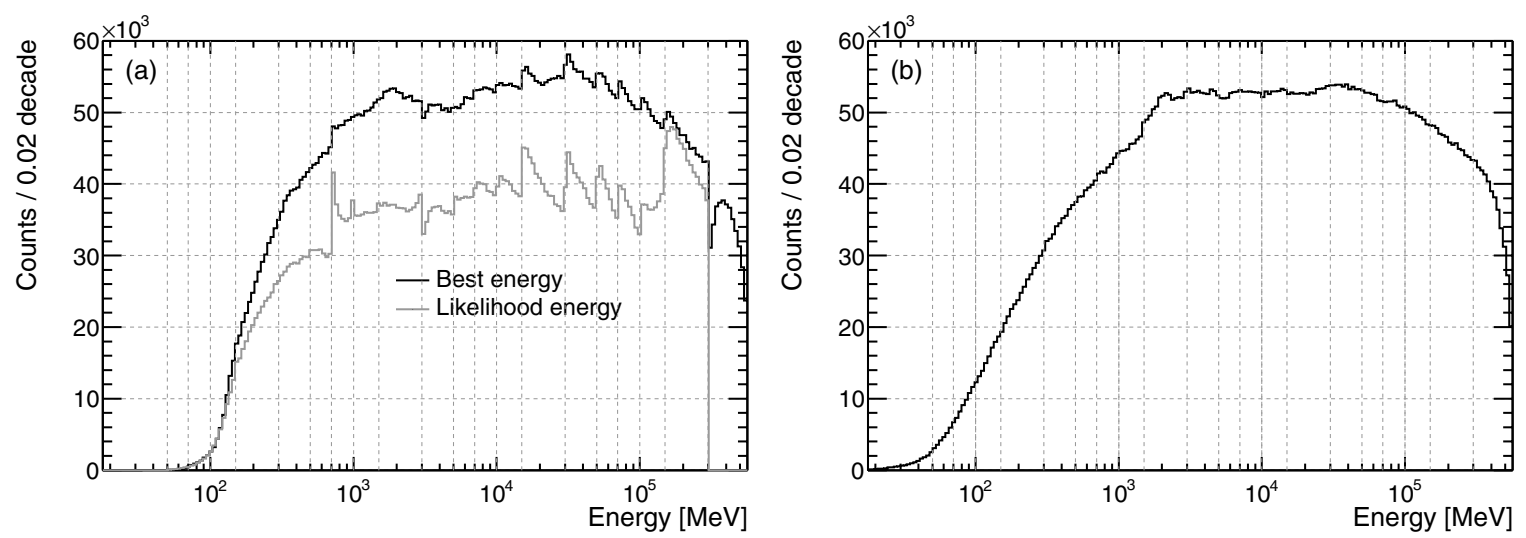

Figure 71. Finely binned (50 bins per energy decade) count spectra for events passing the P6_V3_DIFFUSE (a) and the P7_SOURCE_V6 (b) event selections; (a) also shows the LH energy estimates for the subset of events where they are available (note that the LH energy estimate is not always available, nor is it always selected when it is available). The final energy measurement in Pass 6 reflects the artificial sawtooth structures of the underlying LH energy estimator, which was removed in Pass 7. For both panels the vertical hashing corresponds to the bin boundaries of the LH energy estimator.

specifically designed not to return values above $300 \mathrm{GeV}$. As a consequence, it tends to concentrate events into a relatively narrow feature just below this energy.

Both aspects are illustrated in Figure 71, where count spectra from standard allGamma simulations (Section 2.5.3) are shown for both Pass 6 and Pass 7. While the overall shapes of the spectra reflect the LAT acceptance for the corresponding event classes (P6_V3_DIFFUSE and P7_SOURCE_V6), a clear sawtooth structure is visible in the Pass 6 count spectrum (with a typical width comparable with the LAT energy resolution and peak-totrough amplitude of the order of $\sim 5 \%$ ), along with a prominent feature at $300 \mathrm{GeV}$, above which energies from the LH estimator cannot be selected.

The binning of the count spectra in Figure 71 (50 bins per energy decade) is deliberately much finer than the instrumental resolution-and therefore much finer than the binning one would use for a real spectral analysis of Fermi-LAT data. As a matter of fact, the features of the LH energy estimator have little or no effect in most practical situations. One noticeable exception is the search for spectral lines, such as might occur from the annihilation or decay of a massive particle into a twobody final state including $\gamma$ rays (see Abdo et al. 2010f, for a more detailed discussion).

As already mentioned, in Pass 7 we chose to remove the LH algorithm from consideration in the energy assignment-see Figure 71(b).

\subsection{Uncertainties in the Energy Resolution and Scale}

In this section, we will briefly review the systematic uncertainties on the absolute energy scale and the energy dispersion. The energy measurement is a complex process involving several different steps (see Sections 3.2.1 and 3.3.2). Verification of the process is correspondingly complex, involving tests with sea-level CR muons, tests at accelerators, and analysis of flight data.

\subsubsection{The Calibration Unit Beam Test Campaign}

Since a direct calibration of the LAT with a particle beam was impractical for schedule and cost reasons, the LAT Collaboration assembled for this purpose a dedicated Calibration Unit (CU) composed of two complete flight spare towers, one additional CAL module, and five ACD tiles. An intensive beam test campaign was performed on the $\mathrm{CU}$, between
Table 20

Summary of the 2006 Calibration Unit Beam Test Campaign, Adapted from Baldini et al. (2007)

\begin{tabular}{lccr}
\hline \hline Particle & Line & Energy & Triggers \\
\hline$\gamma$ & PS & $0-2.5 \mathrm{GeV}$ & $12 \mathrm{M}$ \\
$\gamma$ (tagged) & PS & $0.02-1.5 \mathrm{GeV}$ & $4 \mathrm{M}$ \\
$e^{-}$ & PS & $1-5 \mathrm{GeV}$ & $6.4 \mathrm{M}$ \\
$e^{+}$ & PS & $1 \mathrm{GeV}$ & $2.5 \mathrm{M}$ \\
$\pi^{-}$ & PS & $5 \mathrm{GeV}$ & $0.6 \mathrm{M}$ \\
$p$ & PS & $6-10 \mathrm{GeV}$ & $0.6 \mathrm{M}$ \\
\hline$e^{-}$ & SPS & $10-280 \mathrm{GeV}$ & $17.8 \mathrm{M}$ \\
$\pi^{-}$ & SPS & $20 \mathrm{GeV}$ & $1.6 \mathrm{M}$ \\
$p$ & SPS & $20-100 \mathrm{GeV}$ & $0.8 \mathrm{M}$ \\
\hline${ }^{12} \mathrm{C}$ & GSI & $1-1.5 \mathrm{GeV} \mathrm{nucleon}$ & -1 \\
${ }^{131} \mathrm{Xe}$ & GSI & $1-1.5 \mathrm{GeV}$ nucleon & $3 \mathrm{M}$ \\
\hline
\end{tabular}

2006 July and November, with the primary goal of validating the $\mathrm{MC}$ simulation used to define the event reconstruction and the background rejection. The $\mathrm{CU}$ was exposed to beams of bremsstrahlung $\gamma$ rays, protons, electrons, positrons and pions-with energies ranging between $50 \mathrm{MeV}$ and $280 \mathrm{GeV}$ (Table 20)—produced by the CERN Proton Synchrotron (PS) and Super Proton Synchrotron (SPS). In addition, the CU was irradiated with $1-1.5 \mathrm{GeV}$ nucleon ${ }^{-1}{ }^{12} \mathrm{C}$ and ${ }^{131} \mathrm{Xe}$ beams in the Gesellschaft für Schwerionenforschung (GSI) facility with the purpose of studying the instrument response to heavy ions.

A complete review of the results of the beam test campaign is beyond the scope of this paper. The reader is referred to Baldini et al. (2007) for a description of the experimental setup and a review of the main results and to Ackermann et al. (2010) for additional related material.

From the standpoint of the energy measurement one of the primary goals of the beam test campaign was to validate the leakage correction algorithms and, ultimately, the ability of our MC simulation to reliably predict the energy resolution at high energy. The tests confirmed that we understand the overall shower development and the energy resolution to better than $10 \%$ up the maximum available electron energy, i.e., $280 \mathrm{GeV}$ (Ackermann et al. 2010). As we will see in the next section, this implies that the effect of systematic uncertainties on the energy resolution itself, when propagated to the high-level spectral analysis, is essentially irrelevant in the vast majority of practical cases. 
Table 21

Readout Ranges and Energy Conversion Factors for the CAL Crystals, Adapted from Abdo et al. (2009a)

\begin{tabular}{lllcc}
\hline \hline Range & Diode & Gain & Energy Range & MeV Bin $^{-1}$ \\
\hline LEX8 & Small & High & $2 \mathrm{MeV}-100 \mathrm{MeV}$ & 0.033 \\
LEX1 & Small & Low & $2 \mathrm{MeV}-1 \mathrm{GeV}$ & 0.30 \\
HEX8 & Large & High & $30 \mathrm{MeV}-7 \mathrm{GeV}$ & 2.3 \\
HEX1 & Large & Low & $30 \mathrm{MeV}-70 \mathrm{GeV}$ & 20 \\
\hline
\end{tabular}

The most significant discrepancy was observed in the raw energy deposited in the calorimeter, as measured values were on average $\sim 9 \%$ higher when compared with simulations (with smaller fluctuations around this value, slightly dependent on the particle energy and incidence angle). The origin of this discrepancy is unknown, and possibly could be attributed to residual environmental effects not properly accounted for, although an imperfect calibration of the CU cannot be excluded. In Section 7.3.3, we will see that we have indications from flight data that we do understand the absolute energy scale to a precision better than $9 \%$.

\subsubsection{Crystal Calibrations with Cosmic-ray Data}

The calibration of the CAL crystals is the starting point of the energy reconstruction chain and underlies all the subsequent steps (a detailed description of LAT on-orbit calibrations is given in Abdo et al. 2009a). As explained in Section 2.1.2, the large dynamic range of the LAT CAL (2 MeV to $70 \mathrm{GeV}$ per crystal) is achieved by means of four independent chains of electronics per crystal, with different amplifications (or ranges), as summarized in Table 21 . In the most common readout mode (the so-called single-range), the highest gain range that is not saturated is selected as the best estimate and converted into a digital signal that is eventually used in the event reconstruction.

What is relevant for the discussion here is the on-orbit calibration of the crystal response, as determined by the crystal light yield and the linearity of the electronics. The lower energy scales are calibrated using primary protons that do not undergo nuclear interactions in the LAT, which are selected through a dedicated event analysis. For each crystal, the most probable value of the energy deposition, corrected for the path length, is compared with the MC prediction and the conversion factor between digital signal and $\mathrm{MeV}$ is computed. This procedure was first tested and validated with sea-level CR muons. The high-energy ranges are calibrated in the energy range of overlap between LEX1 and HEX8 using events collected by a special on-board filter for selecting heavy ions (see Figure 72), read out in full four-range mode (i.e., with all four energy ranges read out at each log end). The nonlinearity of the electronics is characterized across the entire energy range using a dedicated internal charge injection system and is corrected for in the energy measurement.

Though we originally intended to use heavy primary nuclei for an independent calibration of the high-energy ranges, the uncertainty in the scintillation efficiency of heavy nuclei in the $\mathrm{CsI}(\mathrm{Tl})$ crystals relative to electromagnetic showers makes the heavy ion peaks unsuitable for an independent cross-check of the absolute scale. The magnitude of the effect was measured with 600-1600 MeV nucleon ${ }^{-1} \mathrm{C}$ and $\mathrm{Ni}$ beams (Lott et al. 2006), but we are uncertain how to scale the effect to the typical on-orbit energies ( $\sim 5 \mathrm{GeV}$ nucleon ${ }^{-1}$ or greater) with our desired accuracy of a few percent.

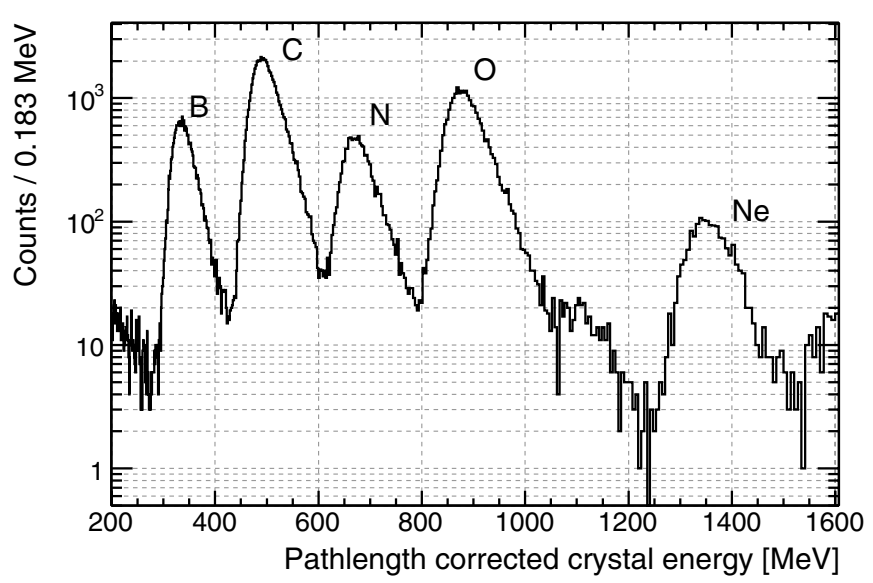

Figure 72. Distribution of the path length-corrected crystal energy deposition for a sample of events used to inter-calibrate the low-energy and high-energy CAL energy ranges. The peaks corresponding to the most abundant Galactic $\mathrm{CR}$ species are clearly visible, though the composition differs somewhat from the Galactic CRs because of secondary production in the ACD and TKR.

We have used protons and Galactic CRs from Be to $\mathrm{Fe}$ at incidence angles ranging from on-axis to $60^{\circ}$ off-axis to demonstrate that the crystal scintillation signal is proportional to path length for each species-i.e., the crystal response is piecewise linear over factors of two in signal. Because the peaks from $\mathrm{Be}$ to $\mathrm{O}$ are closely spaced and easily resolved, this method demonstrates that the CAL energy scale is linear over at least the range $\sim 180 \mathrm{MeV}$ to $\sim 1500 \mathrm{MeV}$ per crystal, so any residual error in absolute energy scale applies equally over that entire range. Unfortunately, it is difficult to bridge the gap between protons at $60^{\circ}(\sim 22 \mathrm{MeV})$ and $\mathrm{He}$ on-axis $(\sim 45 \mathrm{MeV})$, and between $\mathrm{He}$ at $60^{\circ}(\sim 90 \mathrm{MeV})$ and $\mathrm{Be}$ on-axis $(\sim 180 \mathrm{MeV})$, so we cannot demonstrate linearity in this region with this technique. We are exploring alternative CR event selections and geometries to cover these energy regions. Nonetheless, the relative variations of the peak positions (for both non-interacting protons and heavier nuclei) can be measured with high accuracy, which effectively allows us to monitor the time drift of the absolute energy scale and prompt the update of new calibration constants when necessary. The results in Figure 73 are in good agreement with the pre-launch estimates of the crystal light yield attenuation due to radiation damage, which results predominantly from trapped charged particles in the SAA. A fit with the function

$$
s(t)=\left(p_{0}-p_{1}\right)+p_{1} e^{-\frac{\left(t-t_{0}\right)}{\tau}}
$$

yields a time constant $\tau$ of the order of $\sim 2$ years, and predicts an overall shift $\left(\sim p_{0}-p_{1}\right)$ of the energy scale (which can be corrected for) on the order of $4.5 \%$ after 10 years.

\subsubsection{Absolute Measurement of the Energy Scale Using the Earth's Geomagnetic Cutoff}

As mentioned in the beginning of this section, there are essentially no astronomical sources with spectral features that are sharp enough and whose absolute energies are known to such a level of accuracy that they can be effectively exploited for an on-orbit validation of the absolute energy scale.

One exception, perhaps unique in the energy range of the LAT, is the narrow $67.5 \mathrm{MeV}$ pion decay line predicted to originate from interactions of primary CRs with the surface of the Moon (Moskalenko \& Porter 2007). Unfortunately, this feature is located at the lower end of the LAT energy range. While it is 


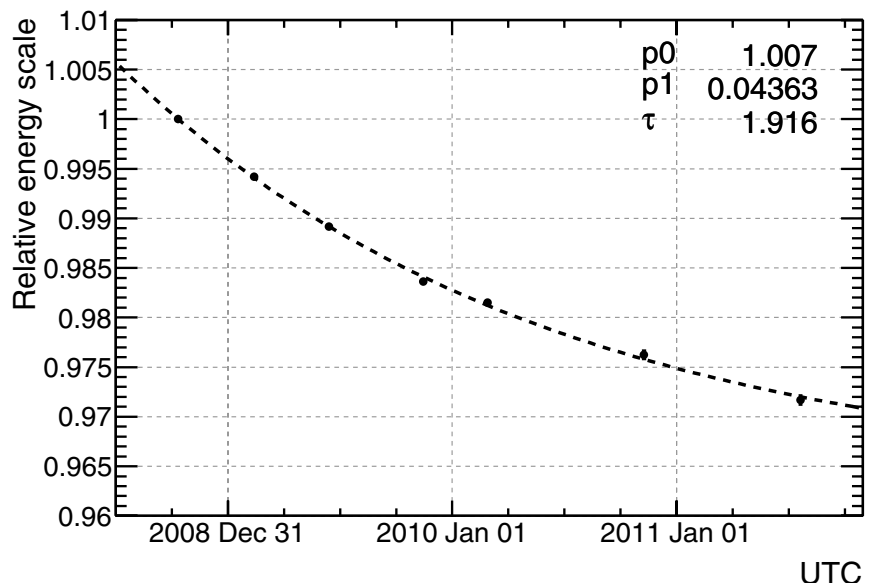

Figure 73. Relative variation of the absolute energy scale, as measured from the position of the proton peak position, throughout the first three years of the mission.

not inconceivable that the LAT possibly could provide the first evidence for the existence of such a line, the limited energy and angular resolution at these energies, together with the brightness of the limb of the Moon in continuum $\gamma$ rays, make the lunar pion line impractical as an absolute energy calibrator.

A practical alternative is the geomagnetic rigidity cutoff in the CR electron spectrum. The LAT is effectively shielded from CRs by the Earth's magnetic field at a low rigidity. The convolution of this shielding with the primary power-law spectrum results in a peaked spectral feature, whose shape and absolute energy can be predicted with good precision by taking advantage of accurate models of the geomagnetic field that are available (Finlay et al. 2010) and particle tracing computer programs (Smart \& Shea 2005). See Section 4.1 for additional details.

Figure 74 shows an example of such predictions in different parts of the Fermi orbit. The count spectra, averaged over the LAT FoV and folded with the LAT energy resolution, are shown in linear, rather than logarithmic, scale in order to emphasize the peaked spectral shape. Comparison of the predicted and measured peak positions is an in-flight measurement of the systematic bias in the energy scale. Since the magnitude of the Earth's magnetic field varies across the Fermi orbit, this approach has the potential to provide a series of calibration points, specifically between $\sim 6 \mathrm{GeV}$ and $\sim 13 \mathrm{GeV}$. The fact that this is the energy range for which the energy resolution of the LAT is the best is beneficial for the measurement itself. Moreover, since both electrons and $\gamma$ rays generate electromagnetic showers in the detector, they are effectively indistinguishable from the calorimeter standpoint-so that energy measurements for one species directly apply to the other.

We have used this approach for an in-flight calibration of the absolute energy scale using one year of data. The details of the analysis are beyond the scope of this paper and are discussed in Ackermann et al. (2012a). The main conclusion is that the measured cutoff energies exceed the predictions by $2.6 \% \pm 0.5 \%$ (stat) $\pm 2.5 \%$ (sys) in the range $6-13 \mathrm{GeV}$.

\subsubsection{Summary of the Uncertainties in the Energy Resolution and Scale}

Here we summarize the results related to the systematic uncertainties of energy measurements with the LAT; in the next section, we will discuss how these impact the high-level spectral analysis.

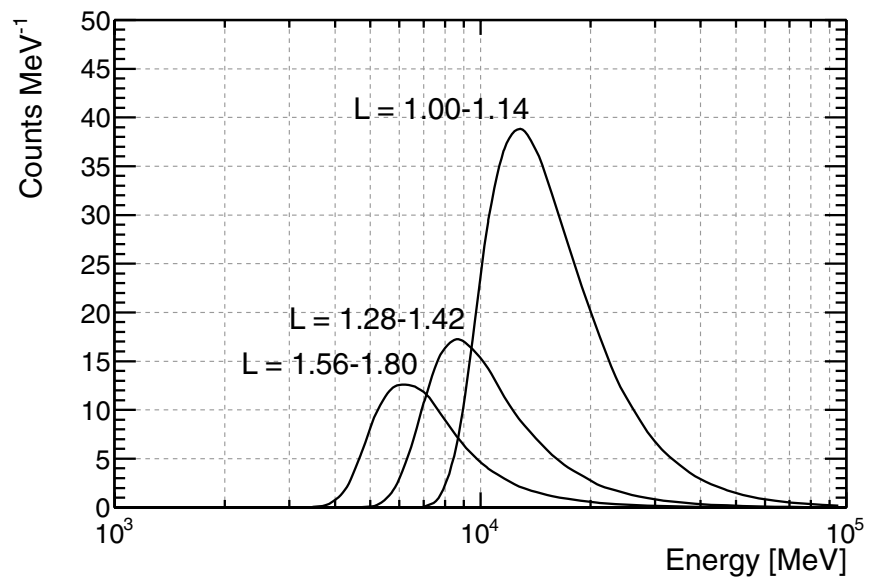

Figure 74. Examples of simulated CR electron count spectra in bins of McIlwain L for the Fermi orbit. They are averaged over the FoV and folded with the energy resolution. Effectively these are the templates that the electron count spectra from real data are compared with in order to measure the absolute energy scale. The peaked shape is the result of the power-law spectrum of primary electrons being convolved with the screening effect of the Earth's magnetic field, which, in each bin of McIlwain L, is effective below a certain cutoff energy. The relative normalizations reflect the fact that Fermi spends the most live time at low McIlwain L.

The results from the $\mathrm{CU}$ beam test campaign indicated that the energy resolution, as predicted by our MC simulations, is accurate to within $10 \%$ up to the maximum accessible beam energy (i.e., $280 \mathrm{GeV}$ ). As we will see in the next section, this implies that this particular systematic effect is negligible in most practical situations.

Though we were not able to identify the reason for the $\sim 9 \%$ discrepancy in the energy deposited in the CU calorimeter measured at the beam tests (Section 7.3.1), we do have compelling indications, from the measurement of the CR electron geomagnetic cutoff (Section 7.3.3), that in fact the systematic error on the absolute energy scale is smaller than that. We stress that this measurement involves the real LAT, with the real flight calibrations, in its on-orbit environment. Although the measurement derived from the CR electron cutoff applies only to a small portion of the LAT energy range, the $\sim 9 \%$ effect measured at the beam test affected essentially the entire range of energy and incidence angle, with a weak dependence on both (see also the remarks in Section 7.3.2 about the additional evidence for the fractional error on the energy scale being constant over a wide energy range). This evidence is further supported by the excellent internal consistency of the analysis measuring the electron and positron spectra separately (Ackermann et al. 2012b). However, it is more difficult to constrain the energy scale at the low and high ends of the LAT energy range, where the energy resolution degrades by a factor of $\sim 2$. Based on the full body of information currently available we conclude that that the energy scale for the LAT is correct to $+20 \% /-50 \%$ of the energy resolution of the LAT at a given energy. This corresponds to an uncertainty of $+2 \% /-5 \%$ on energy scale over the range $1-100 \mathrm{GeV}$, and increases to $+4 \% /-10 \%$ below $100 \mathrm{MeV}$ and above $300 \mathrm{GeV}$.

Finally, the measured energies of the Galactic CR peaks are being monitored to gauge the time stability of the absolute energy scale, which we can control at the $1 \%$ level by applying calibration constants on a channel-by-channel basis. 


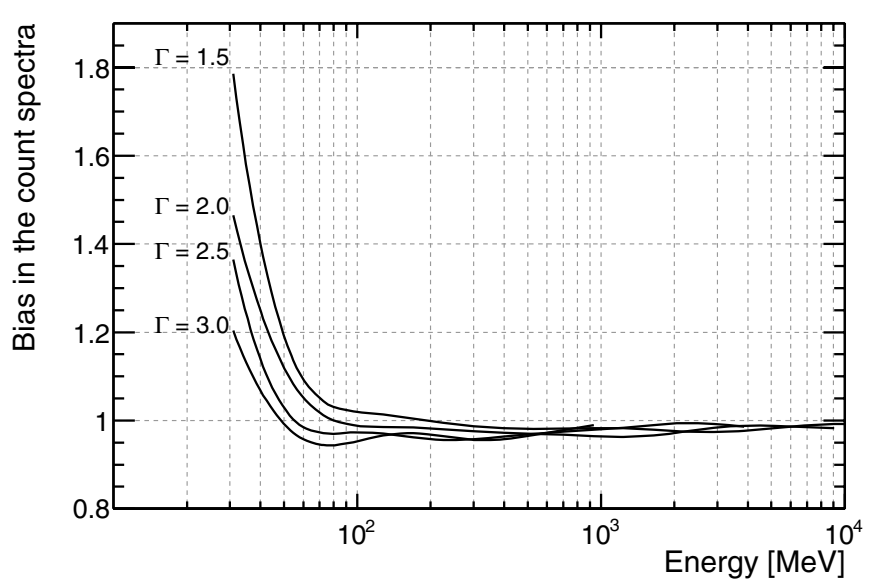

Figure 75. Bias factor for count spectra induced by not taking into account the finite energy resolution of the LAT. The plots refer to simulated point sources (with no background) with power-law spectra, for several different values of the spectral index.

\subsection{Propagating Systematic Uncertainties of the Energy Resolution and Scale to High-level Science Analyses}

Uncertainties in the energy resolution and scale introduce systematics in spectral analyses, the magnitude of which depends on energy and the spectral shape of the sources under study. Since these systematic effects are a consequence of event redistribution between energy bins, effects are also strongly coupled to the energy dependence of $A_{\text {eff }}$. As the implications of the systematic uncertainties are so dependent on the analysis, the main purpose of this section is to illustrate the basic ideas and the tone of the discussion is deliberately general.

The effect of the finite energy resolution is generally negligible for spectral analysis above $\sim 100 \mathrm{MeV}$, which as already mentioned, is one of the main reasons why it is not taken into account in the standard likelihood analysis. Even above $\sim 100 \mathrm{GeV}$, where the energy resolution is significantly degraded by the leakage of the shower from the CAL, the effect is negligible compared with other sources of systematics-at least for sources with steep spectra, like primary CR electrons (Ackermann et al. 2010). MC studies with simulated point sources, though, show that the finite resolution can induce a bias in the count spectra as high as $20 \%-30 \%$ at $50 \mathrm{MeV}$ for power-law spectra with $\Gamma \sim 1.5$. The effect is strongly dependent on the spectral index and is less severe for softer sources, as shown in Figure 75.
Table 22

Systematic Variations Arising from Ignoring the Effects of Energy Dispersion When Performing Likelihood Fitting

\begin{tabular}{lcc}
\hline \hline Parameter & \begin{tabular}{c} 
B2 $\begin{array}{c}1520+31 \\
\text { (Soft) }\end{array}$ \\
\hline$\delta N_{0} / N_{0}$
\end{tabular} & $\begin{array}{c}\text { PG } 1553+113 \\
\text { (Hard) }\end{array}$ \\
$\delta \Gamma \delta \alpha$ & $+3.9 \%$ & $+2.2 \%$ \\
$\delta \beta$ & -0.04 & +0.01 \\
$\delta F_{25} / F_{25}$ & +0.02 & $\ldots$ \\
$\delta S_{25} / S_{25}$ & $+1.4 \%$ & $+3.3 \%$ \\
\hline
\end{tabular}

Notes. For the spectral index ( $\Gamma$ or $\alpha$ ) and spectral curvature $(\beta)$, we give the absolute variation with respect to the value obtained when ignoring the energy dispersion (e.g., $\delta \Gamma$ ). For the flux prefactor and the integral fluxes, we give the relative variation with respect to the value obtained when ignoring the energy dispersion (e.g., $\delta N_{0} / N_{0}$ ).

A bias in a count spectrum, in general, does not trivially translate into an effect of the same order of magnitude in the parameter values derived from a spectral analysis. When fitting a source spectrum with a single power law, for instance, the combination of the long lever arm of the high-energy data points and the inability of the spectral model to accommodate any curvature in the count spectrum, results in the energy dispersion having very little effect on the fit parameters (even though it can produce very significant residuals). Figure 76(a) shows that in this setup the bias in the measured spectral index, when fitting down to $30 \mathrm{MeV}$, is smaller than 0.03 for any reasonable input spectrum.

The situation can be quite different for other spectral shapes-e.g., a power-law spectrum with exponential cutoff, as illustrated in Figure 76(b). Particularly, the bias introduced by the energy dispersion reaches in this case $\sim 0.15$ for hard spectral indices. The problem can be studied, for a specific source model, by means of dedicated MC simulations, and this is strongly recommended, especially for spectral analysis below $100 \mathrm{MeV}$. Generally speaking, enabling the energy dispersion handling in the standard binned likelihood analysis significantly reduces the bias in the fit parameters.

To quantify the magnitude of the bias introduced by ignoring energy dispersion in the likelihood fit we have re-analyzed both the B2 $1520+31$ and the PG $1553+113$ ROIs with the energy dispersion treatment enabled using ScienceTools (version v9r28p0). We list the changes with respect to the fit results with the energy dispersion ignored in Table 22.
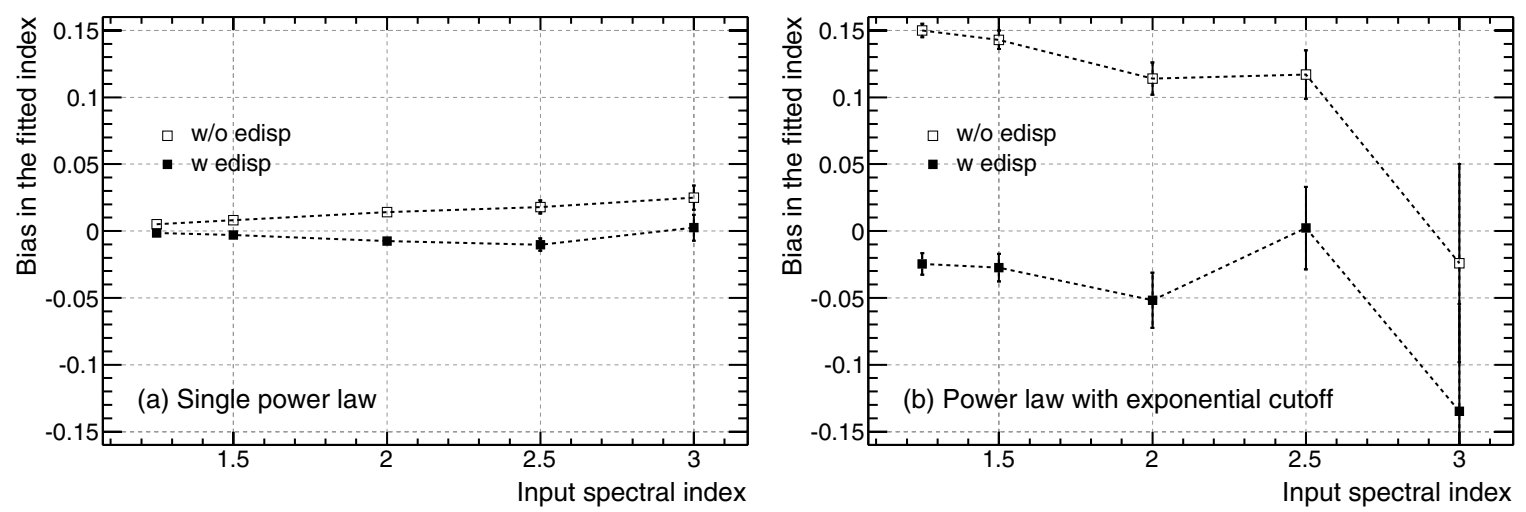

Figure 76. Bias in the spectral index returned by a binned likelihood analysis with a minimum energy of $30 \mathrm{MeV}$ (this setup is taken as a worst case scenario; increasing the minimum energy for the fit to $100 \mathrm{MeV}$ decreases the bias by a factor of two or more). The plots refer to simulated point sources (with no background) with two different spectral shapes - a single power law (a) and power law with an exponential cutoff at $1 \mathrm{GeV}$ (b) - for several different values of the input index. Enabling the energy dispersion handling in ScienceTools significantly reduces the bias in the fit parameters. 
Table 23

Systematic Variations of Integral Fluxes Arising from Uncertainties in the Absolute Energy Scale

\begin{tabular}{lcc}
\hline \hline Parameter & $\begin{array}{c}\text { B2 } 1520+31 \\
\text { (Soft) }\end{array}$ & $\begin{array}{c}\text { PG } 1553+113 \\
\text { (Hard) }\end{array}$ \\
\hline$\delta F_{25} / F_{25}$ & $+13.4 \%-4.6 \%$ & $+3.4 \%-2.5 \%$ \\
$\delta S_{25} / S_{25}$ & $+3.8 \%-1.4 \%$ & $+1.7 \%-0.6 \%$ \\
\hline
\end{tabular}

Note. We give the relative variation with respect to the nominal value (e.g., $\delta F_{25} / F_{25}$ ).

MC simulations also show that the effects of systematic uncertainties in the energy resolution, at the level we understand the energy dispersion of the detector, are essentially negligible over the entire energy range when studying $\gamma$-ray sources that do not have sharp spectral features-which accounts for the vast majority of cases of practical interest. One noticeable exception is the search for $\gamma$-ray lines, which requires a dedicated analysis when evaluating limits. But even in that case the systematic uncertainty on the energy resolution is not a major source of concern: if the actual energy resolution was $\sim 10 \%$ broader than that predicted by the MC the fitted signal counts would be $\sim 10 \%$ lower, i.e., not enough to dramatically decrease the sensitivity to a spectral line.

The effect of systematic uncertainties on the absolute energy scale is also strongly dependent on the energy range of interest. In the ideal case of an energy-independent effective area, it can be shown that, for a power-law spectrum with index $\Gamma$, a relative bias $b$ in the absolute scale translates into a rigid shift of the spectrum itself by the amount $\Delta F / F=(\Gamma-1) b$ (or $\Delta S_{25} / S_{25}=(\Gamma-2) b$ if we consider the integral energy flux). In short, the measurement of the spectral index is not affected-while the flux obviously is.

As a concrete example, we estimate the effect of systematic uncertainties of the absolute energy scale on the integral fluxes $F_{25}$ and $S_{25}$ of PG $1553+113$ and B2 $1520+31$ by integrating the fitted source spectra and shifting the limits of integration by the uncertainties stated in Section 7.3.4. Specifically, we consider the integration ranges $96 \mathrm{MeV}$ to $95 \mathrm{GeV}$ and $104 \mathrm{MeV}$ to $102 \mathrm{GeV}$; the resulting uncertainty estimates are reported in Table 23.

Along the same lines, measured cutoff energies for sources with curved spectra reflect the bias in the energy scale directly: $\Delta E_{c} / E_{c}=b$. These statements are useful rules of thumb to estimate the order of magnitude of the effect above $\approx 1 \mathrm{GeV}$, where the effective area is not strongly dependent on energy (Figure 77). In fact, since we have good indications that we understand the energy scale of the detector at the $\sim 5 \%$ level (see Sections 7.3.1 and 7.3.3) at these energies, the effect of systematic uncertainties in the scale is generally smaller than other sources of systematics in this energy range, and does not affect the measurement of the spectral index in the power-law case.

Below a few hundred $\mathrm{MeV}$ the systematic uncertainties in the absolute energy scale should be carefully considered in any analysis due to the unfortunate combination of a steeply increasing effective area and a worsening of the energy resolution at low energies. As for the energy dispersion the effect is more pronounced for steep sources. For the typical case of a spectral index of $\Gamma \approx 2, \mathrm{a} \pm 5 \%$ uncertainty in the energy scale is negligible down to $\sim 100 \mathrm{MeV}$ but at $50 \mathrm{MeV}$ can easily translate into an additional $10 \%-20 \%$ bias in the count spectrum. Again, the order of magnitude of the effect can be easily assessed on

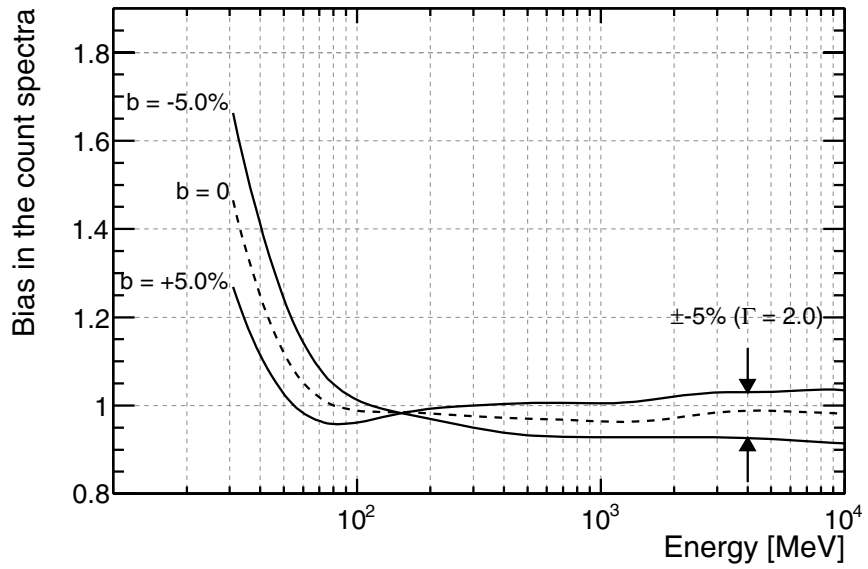

Figure 77. Bias factor on the count spectra induced by systematic uncertainties on the absolute energy scale for a simulated point source (with no background) with power-law spectrum with $\Gamma=2$. The parameter $b$ indicates the relative bias in the absolute energy scale; the case $b=0$ corresponds to the line at $\Gamma=2$ in Figure 75.

a case-by-case basis, depending on the spectral shape of the source under study and energy range of interest.

Spectral unfolding (deconvolution) is an approach for taking into account the energy dispersion in the spectral fitting. Unfolding is in effect the opposite of the forward folding approach implemented in many popular astronomical data analysis packages (and used in the standard likelihood point-source analysis implemented in ScienceTools), where the IRFs are folded with the spectral model and the model parameters are varied until the best match with the measured count spectrum is found-e.g., this is how XSPEC (Arnaud 1996) handles the energy dispersion.

Energy unfolding has been used for several analyses of LAT data, mainly to cross-check the results of the standard likelihood analysis (Abdo et al. 2010a) and especially for the lowest or highest energies: below $\sim 100 \mathrm{MeV}$ (Abdo et al. 2010d) or above $\sim 100 \mathrm{GeV}$ (Ackermann et al. 2010; Abdo et al. 2009b). The Bayesian approach detailed in D'Agostini (1995) is that typically being used by the LAT Collaboration.

\subsection{Event Analysis-induced Spectral Features}

Though we discuss potential event analysis-induced spectral features in the context of energy reconstruction, we emphasize that the effective area is also germane to this topic. We conclude that the event analysis and IRFs do not introduce significant artificial spectral features because bright $\gamma$-ray sources are devoid of spectral features - particularly the Earth limb (Section 3.6.3), which is the single brightest source in the LAT energy range.

Figure 78 shows a count spectrum of P7CLEAN_V6 $\gamma$ rays from the Earth limb based on the first two years of data. The spectrum is made with $\sim 16$ bins per energy decade-corresponding to a bin width slightly smaller than the typical LAT energy resolution-and its smoothness is a good qualitative indicator of the smallness of any possible instrument-induced spectral feature.

In order to quantify this we compared the counts $n_{i}$ in the $i$ th energy bin with the value $f_{i}$ returned by log-parabola fit to the four nearest bins (i.e., two bins on each side). We can construct two obvious metrics, namely the normalized residuals

$$
r_{i}^{n}=\frac{\left(n_{i}-f_{i}\right)}{\sqrt{f_{i}}},
$$




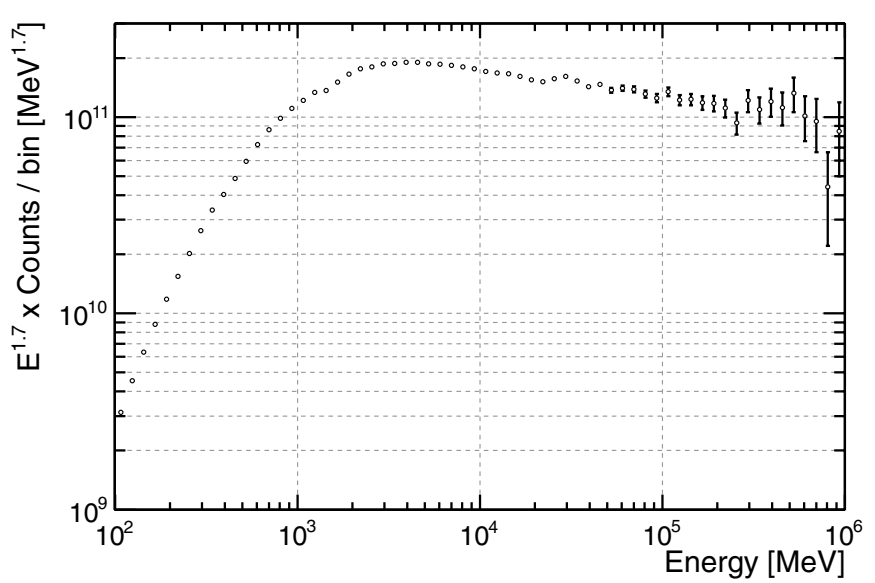

Figure 78. Count spectrum of the Earth limb based on the first two years of data, including P7CLEAN_V6 events within three times the acceptance-averaged PSF $68 \%$ containment from the nominal zenith angle of the limb. Since the limb itself is typically observed at large incidence angle in the instrument frame, events impinging on the LAT more than $65^{\circ}$ off-axis have been removed in order not to overweight the edge of the FoV relative to typical analyses of celestial sources. The turnover below $\sim 1 \mathrm{GeV}$ is mostly due to the decrease of the LAT acceptance, rather than to an intrinsic roll-off of the source spectrum.

and the fractional residuals

$$
r_{i}^{f}=\frac{\left(n_{i}-f_{i}\right)}{f_{i}} .
$$

Figure 79 shows a scatter plot of the two metrics, each point corresponding to an energy bin in the count spectrum in Figure 78. There are two striking features: (1) a nearly horizontal branch corresponding to the energy bins below $\sim 1 \mathrm{GeV}$, where the statistics are large and the curvature of the count spectrum makes the quadratic fits less accurate, and (2) a nearly vertical branch, corresponding to the high-energy points, whose relatively larger fractional deviations (up to $\sim 10 \%$ ) are just due to statistical fluctuations. We note for completeness that there are no points farther than $5 \sigma$ from the fit value above $2 \mathrm{GeV}$, which implies that we do not expect to be able to see artificial spectral features above this energy in any practical case. At lower energies the high counting statistics of the Earth limb emission allows placing an upper limit on possible narrow (i.e., less than about twice the energy resolution) spurious features at the level of a few percent. This method for setting limits on spurious line features is decreasingly sensitive to broader features, which are more likely to arise from correlated errors in $A_{\text {eff }}$ (see Section 5.6.2).

\subsection{Comparison of Pass 6 and Pass 7}

Though the underlying energy reconstruction algorithms are exactly the same, Pass 7 differs from Pass 6 in (1) the new unbiasing stage described in Section 3.2.1 and (2) the fact that the LH estimator is no longer used in the event-level analysis for the final energy assignment (see Section 3.3.2).

Figure 80 shows that the energy resolution for the P7SOURCE_V6 event class is quite comparable to that of P6_V3_DIFFUSE over most of the LAT energy range. The most noticeable difference, namely a slight worsening below $1 \mathrm{GeV}$ (and especially below $100 \mathrm{MeV}$ ), is a small tradeoff for the much greater Pass 7 low-energy acceptance. On the other hand, Pass 7 has uniformly better energy resolution than Pass 6 in the energy range above $10 \mathrm{GeV}$.

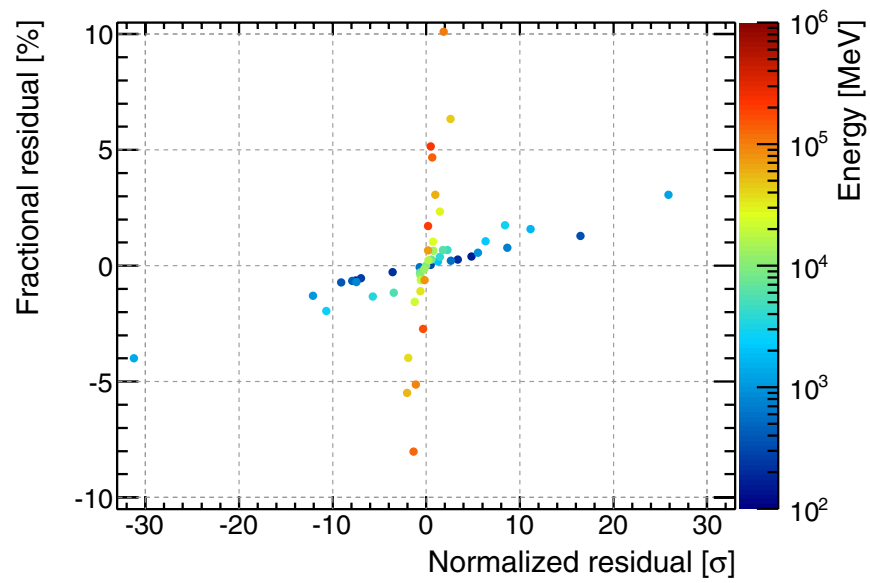

Figure 79. Single-point residuals of the count spectrum in Figure 78 with respect to a log-parabola fit of the four nearest points.

(A color version of this figure is available in the online journal.)

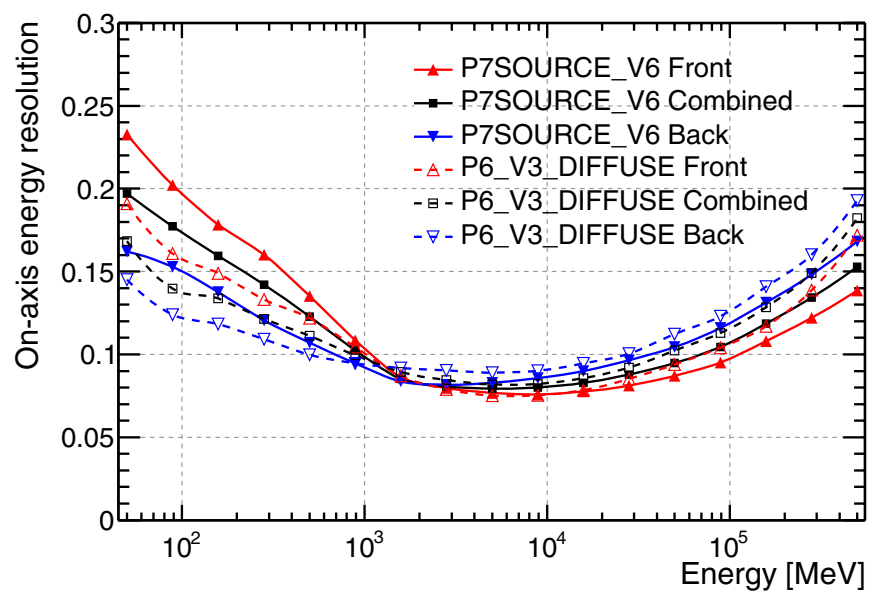

Figure 80. Comparison of the on-axis energy resolutions of the P7SOURCE_V6 and P6_V3_DIFFUSE IRFs.

(A color version of this figure is available in the online journal.)

\section{PERFORMANCE FOR HIGH-LEVEL SCIENCE ANALYSIS}

We report here the high-level science analysis performance of the LAT for the Pass 7 event selections.

\subsection{Point-source Sensitivity}

A detailed description of the point-source sensitivity of the LAT is given in Abdo et al. (2010b) and Nolan et al. (2012). In particular, following the procedure described in Abdo et al. (2010b), a semi-analytical estimate of the LAT sensitivity for point sources can be calculated for the Pass 7 event analysis. Here we calculate the LAT sensitivity to a point source for the P7SOURCE_V6 IRFs - as described in Abdo et al. (2010b) for P6_V3-under the following assumptions.

1. Power-law spectrum with $\Gamma=2$.

2. Diffuse Galactic emission as described in the twoyear Galactic diffuse model, publicly distributed as gal_2yearp7v6_v0.fits.

3. Diffuse isotropic background as described in the two-year template, publicly distributed as iso_p7v6source.txt.

4. No confusion with nearby sources. 

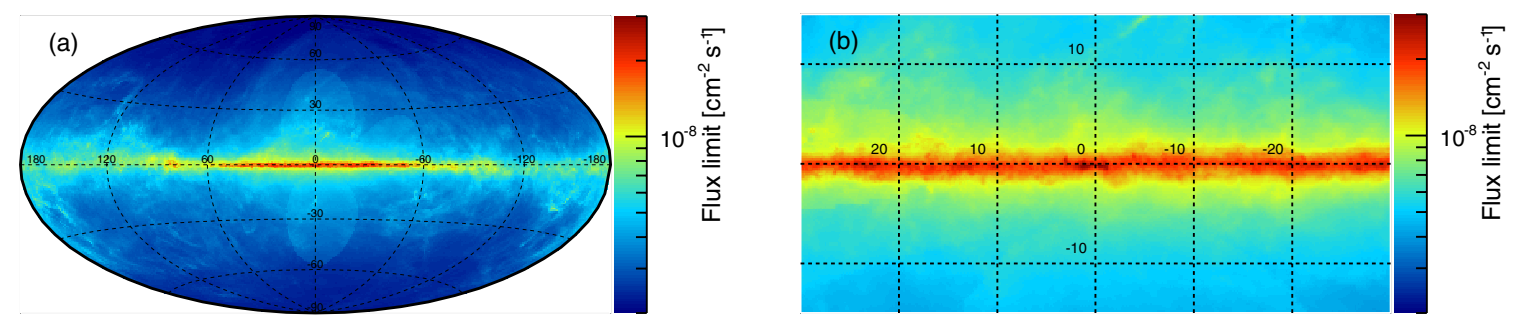

Figure 81. Flux above $100 \mathrm{MeV}$ required for $5 \sigma$ sensitivity for the P7SOURCE_V6 event class for a point source with power-law spectrum with index $\Gamma=2$. The calculation assumes a three-year exposure. The entire sky (a) and a zoom on the Galactic center (b) are shown.

(A color version of this figure is available in the online journal.)

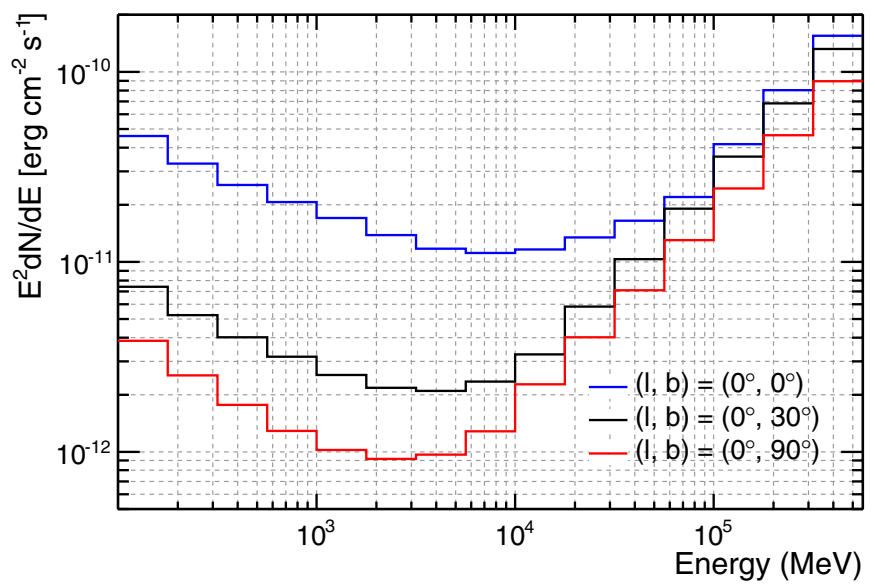

Figure 82. Differential sensitivity for class P7SOURCE_V6 for a point source; calculation for three-year exposure, four bins per energy decade. Requirements are $5 \sigma$ sensitivity and at least 10 counts per bin. The sensitivity is calculated at three locations in the sky: at the Galactic pole, at an intermediate latitude and on the Galactic plane.

(A color version of this figure is available in the online journal.)

5. Exposure calculated for the first three years of nominal science operations.

A map of the flux limit is shown in Figure 81. Away from the Galactic plane the sensitivity is rather uniform. In reality, the presence of bright point sources will affect the flux limit and a dedicated analysis is recommended to evaluate the limit for any particular circumstance.

In Figure 82, we show the corresponding differential sensitivity curves. The curves show the flux limits for narrow energy ranges and illustrate the sensitivity for spectral measurements as a function of energy. We require that in each energy band the source be bright enough to have $5 \sigma$ detection and cause at least $10 \gamma$ rays to be collected.

Finally, we stress that along the Galactic plane the uncertainties of the Galactic diffuse emission can affect the detection significance of a source, and structured residuals in the Galactic diffuse model can be mischaracterized as point sources. Further discussion can be found in Fermi-LAT point-source catalogs and papers about the Galactic diffuse emission (Abdo et al. 2010b; Nolan et al. 2012; Ackermann et al. 2012d).

\subsection{Point-source Localization}

The performance of the LAT for the localization of a point source at the detection threshold also can be evaluated with a semi-analytical approach. The estimated two-year performance (see Nolan et al. 2012) indicates that the $95 \%$ localization radius for an isolated point source detected at $5 \sigma$ significance at high

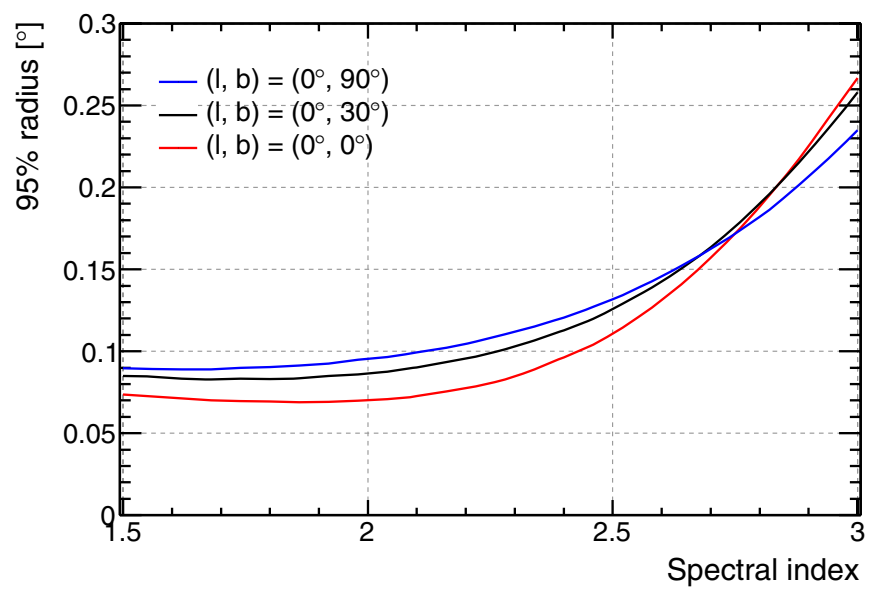

Figure 83. 95\% localization radius for class P6SOURCE_V6 for a point source at the detection threshold with power-law spectrum, as a function of the spectral index (three-year exposure for different locations in the sky). For sources in the Galactic plane detected at a given significance, the localization performance for those sources which are softer than the Galactic diffuse background (which consists of spectral components $\Gamma \in[2.1,2.7])$ is significantly degraded with respect to harder sources.

(A color version of this figure is available in the online journal.)

Galactic latitude ranges from $\sim 0.1$ for a hard spectral index $(\Gamma=1.5)$ to $\sim 0.3$ for a soft spectral index $(\Gamma=3.0)$.

Scaling the source location determinations to more intense background levels is not straightforward. First of all, localization regions are usually elliptical in shape, as described in Nolan et al. (2012). Second, as the astrophysical background increases, e.g., in the Galactic plane, for a given value of the spectral index, a higher flux is needed to reach the detection threshold. As a consequence, the size of the localization region, which is more sensitive to the number of high-energy $\gamma$ rays than the pointsource sensitivity, will be smaller within the Galactic plane. This apparently counterintuitive result is shown in Figure 83, where the $95 \%$ localization radius for a power-law source is shown as a function of the spectral index for three different locations. The improvement of the localization radius with time (Figure 83) is of the order of $10 \%$ or less going from a three-year to a five-year exposure.

Nolan et al. (2012) compared the positions and error regions of $\gamma$-ray sources to the positions of associated multiwavelength counterparts and empirically corrected the localization uncertainties by multiplying the error region by a 1.1 scale factor and adding 0.005 in quadrature to the $95 \%$ error ellipse axes. Although the uncertainties of the Galactic diffuse emission are significantly larger along the Galactic plane, we did not observe any systematic increase in the localization offsets with respect to associated multiwavelength counterparts in the Galactic plane. 

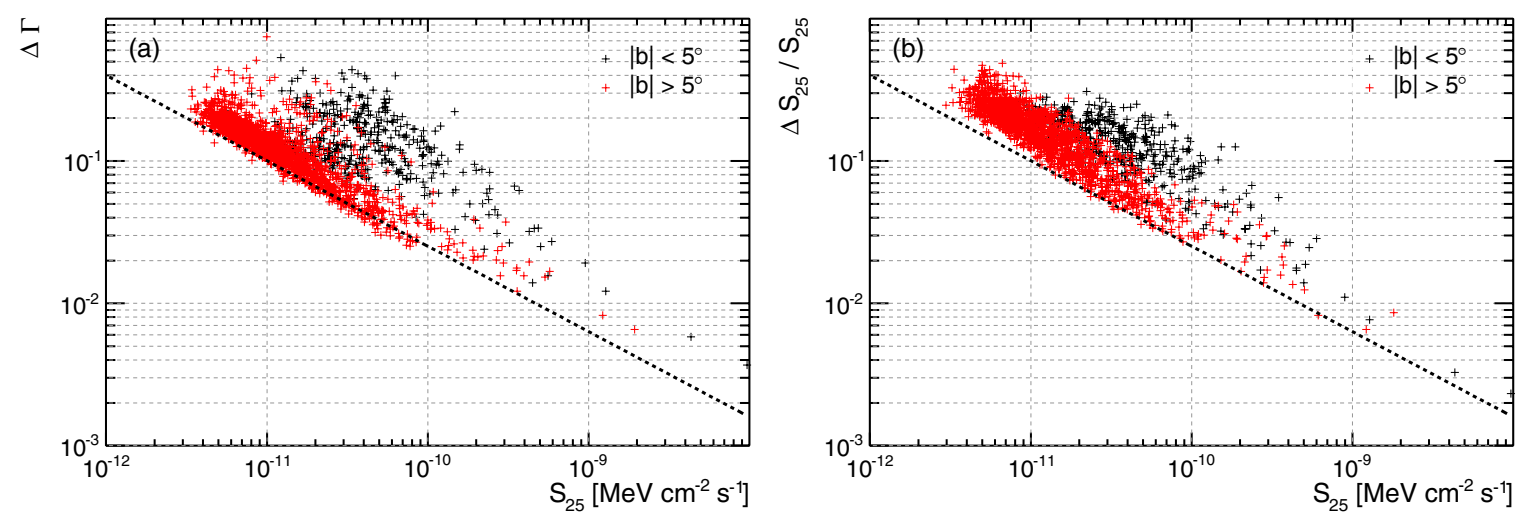

Figure 84. Statistical uncertainties on spectral index $\Gamma$ (a) and integral energy flux above $100 \mathrm{MeV} S_{25}$ (b) as a function of $S_{25}$ for all sources in the $2 \mathrm{FGL}$ catalog (Nolan et al. 2012). The dashed lines show a $S_{25}^{-0.6}$ dependence for comparison.

(A color version of this figure is available in the online journal.)
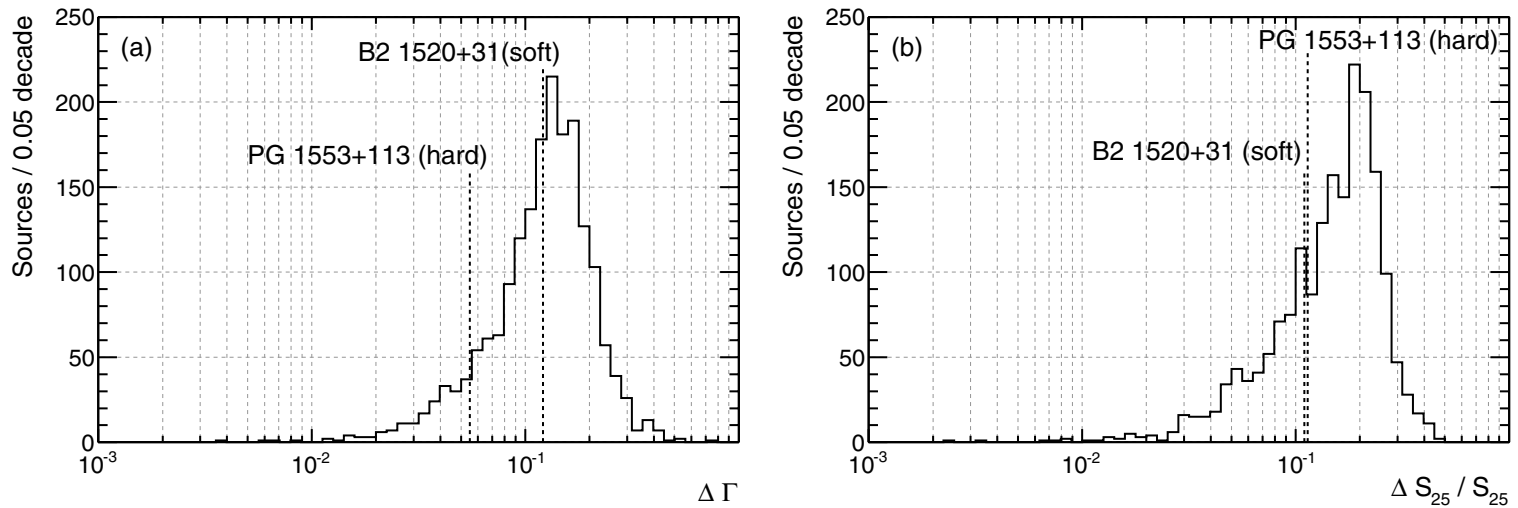

Figure 85. Statistical uncertainties on $\Gamma$ (a) and $S_{25}$ (b) for all 2FGL sources. For comparison, the estimated systematic uncertainties for B2 $1520+31$ and PG $1553+113$ are also shown.

\subsection{Flux and Spectral Measurements}

The precision to which we can measure the flux and spectral index of any source depends primarily on the counting statistics, which in turn depends on the flux of the source. However, since the width of the PSF decreases with energy, high-energy $\gamma$ rays contribute more to source detection, so given similar energy fluxes, we can measure harder sources more precisely. Finally, the flux of the source relative to nearby diffuse backgrounds and other nearby sources will also limit the precision of the parameter measurements in a likelihood analysis. Figure 84 shows the dependence of the statistical uncertainties of spectral index $\Gamma$ and the integral energy flux between $100 \mathrm{MeV}$ and $100 \mathrm{GeV}\left(S_{25}\right)$ for the sources in the 2FGL catalog (Nolan et al. 2012). In both cases, the minimum statistical uncertainty decreases as roughly $S_{25}^{-0.6}$.

For comparison, we combine the systematic uncertainty estimates based on the bracketing tables for the effective area (Section 5.7), the PSF (Section 6.5), and the effect of ignoring the energy dispersion (Section 7.4) for the two sources we studied in detail (B2 1520+31 and PG 1553+113). Since we have not seen any evidence of correlated biases in the different terms of the IRFs (see, e.g., Section 7.1.4), we simply combine the uncertainties in quadrature, as summarized in Table 24.

Figure 85 shows the distributions of the statistical uncertainties on $\Gamma$ and $S_{25}$ from the 2FGL catalog, along with our estimates of the typical systematic uncertainties for hard and soft sources based on our analysis of B2 1520+31 and PG 1553+113. For the majority of sources in the 2FGL catalog the measurement
Table 24

Rough Estimates of the Magnitude of the Effects of Various Sources of Systematic Errors on the Integral Energy Flux and Spectral Index of B2 $1520+31$ and PG $1553+113$

\begin{tabular}{lccccc}
\hline \hline Quantity & \multirow{2}{*}{$A_{\text {eff }}$} & PSF & \multicolumn{2}{c}{ Energy } & Total \\
\cline { 3 - 5 } & & & Dispersion & Scale & \\
\hline$\delta \Gamma_{\mathrm{PG} 1553+113}$ & 0.05 & 0.02 & 0.01 & $\ldots$ & 0.05 \\
$\delta \Gamma_{\mathrm{B} 21520+31}$ & 0.09 & 0.07 & 0.04 & $\ldots$ & 0.12 \\
$\frac{\delta S_{25}}{S_{25}} \mathrm{PG} 1553+113^{\delta}$ & $10 \%$ & $5 \%$ & $1 \%$ & $2 \%$ & $11 \%$ \\
$\frac{\delta S_{25}}{S_{25} \mathrm{~B} 21520+31}$ & $8 \%$ & $6 \%$ & $2 \%$ & $4 \%$ & $11 \%$ \\
\hline
\end{tabular}

precision is still limited by the statistical uncertainties, though for the brightest sources the systematic uncertainties dominate. For both $\Gamma$ and $S_{25}$ the transition from statistical limitation to systematic limitation occurs for $S_{25} \sim 10^{-11} \mathrm{erg} \mathrm{cm}^{-2} \mathrm{~s}^{-1}$.

We emphasize that the results in this section were derived using bracketing IRFs designed to maximize the variation of the fit results for the particular sources under study. As such, they represent the systematic uncertainties of measurements on any single source. Since measurements of different sources share the same IRFs and associated uncertainties, any relative comparison between measurements is significantly more precise.

Finally, along the Galactic plane the uncertainties of the Galactic diffuse emission can affect measurements of source parameters and spectral indices. As with the question of how these uncertainties affect the LAT point-source detection sensitivity, detailed discussion is beyond the scope of this paper, but 

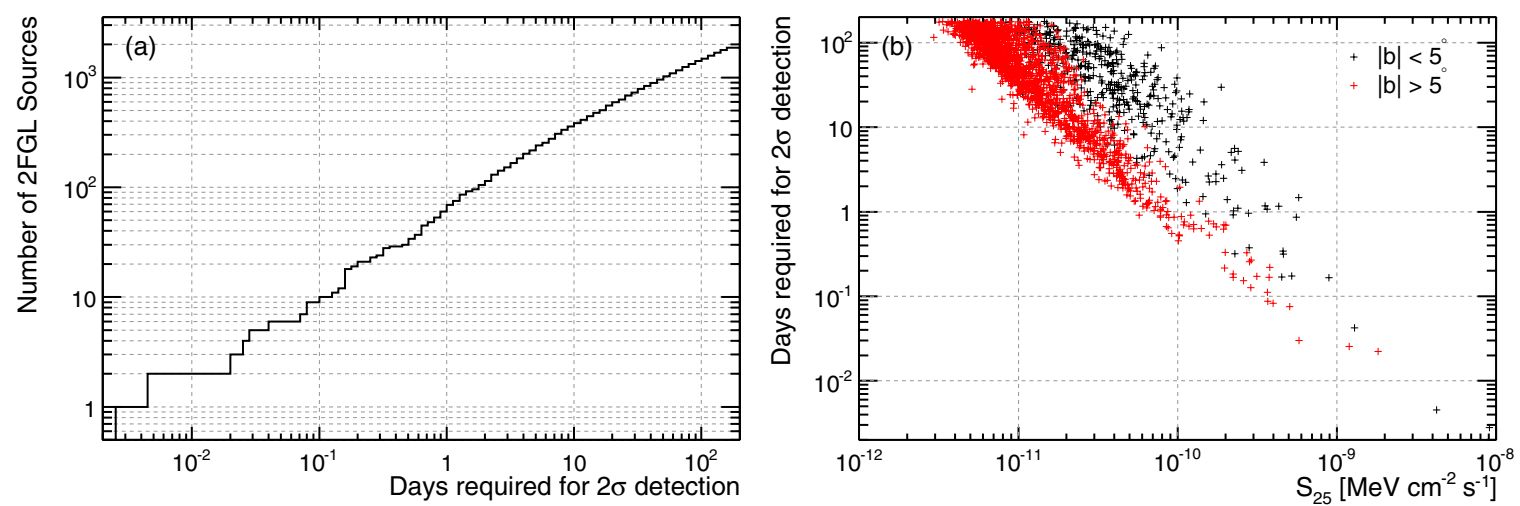

Figure 86. LAT sensitivity to variability of all 2FGL catalog sources. Panel (a) shows the cumulative distribution of the estimator $\tau_{2 \sigma}$, an estimate of the time needed to detect a steady source with $2 \sigma$ significance. Panel (b) shows $\tau_{2 \sigma}$ as a function of the integral energy flux between $100 \mathrm{MeV}$ and $100 \mathrm{GeV}\left(S_{25}\right)$ for both low and high Galactic latitude sources.

(A color version of this figure is available in the online journal.)

can be found in Abdo et al. (2010b), Nolan et al. (2012), and Ackermann et al. (2012d).

\subsection{Variability}

The LAT's ability to measure the variability of any given source depends on the characteristics of both the source and the astrophysical backgrounds.

We can use the detection significances of sources in the 2FGL catalog to derive a rough estimate of the timescales at which we can probe the variability of those sources. For detecting variability, the worst-case scenario is a steady source, so we ask how long would be required on average to detect any source at a particular threshold, assuming steady emission. Specifically, we can construct a metric $\tau_{2 \sigma}$, the time required to achieve $2 \sigma$ detection of a source, assuming steady emission at the average 2FGL level, which can be expressed in terms of the significance of the source in the $2 \mathrm{FGL}$ catalog $\left(\sigma_{2 \mathrm{FGL}}\right)$ and the amount of data used to construct the catalog $(\sim 730$ days):

$$
\tau_{2 \sigma} \sim 4 \sigma_{2 \mathrm{FGL}}^{-2} 730 \text { days. }
$$

Figure 86 shows this estimator for all 2FGL sources, we emphasize that we are sensitive to more significant flux changes on timescales shorter than $\tau_{2 \sigma}$.

In Sections 5.6.3 and 6.5.3 we examined how instrumental uncertainties in the $A_{\text {eff }}$ and PSF can change over time, and could potentially induce artificial variability in the measurement of point-source fluxes.

More specifically, in the case of the $A_{\text {eff-related variability }}$ we showed that the phase-gated counts excess was extremely stable, with the Fourier transform of $\delta n=n-\tilde{n}$ showing only a single small feature consistent with the 53.4 day orbital precession period. However, since that analysis did not treat the PSF, nor allow for the effect of changing statistics from differing exposures on the Fourier transform of $\delta n$, we could not use it to predict the level of instrumentally induced variability we might measure in a likelihood-based analysis using ScienceTools.

Similarly, our analysis of PSF-related variability estimated how much the containment as defined by the $\theta$-averaged $R_{68}$ and $R_{95}$ might vary over a precession period, but did not attempt to quantify the effect on a likelihood-based analysis using ScienceTools. In order to quantify these effects, we have studied the Vela and Geminga (PSR J0633+1746; Abdo et al. 2010h) pulsars in $12 \mathrm{hr}$ time bins with the ScienceTools (version
09-26-02) unbinned likelihood analysis and the following analysis parameters.

1. We started with the $\gamma$-ray and time interval selection criteria as for the Vela calibration sample (see Section 3.6); for the Geminga sample we used the same $15^{\circ}$ radius for the ROI.

2. We tightened the zenith angle requirement to $\theta_{z}<95^{\circ}$ and used all $\gamma$ rays with energies $E>70 \mathrm{MeV}$.

3. We included all 2 FGL sources within $20^{\circ}$ in our likelihood model, with the same spectral parameterizations as were used in the 2FGL catalog.

4. We included models of the Galactic diffuse emission (ring_2year_P76_v0.fits) and the isotropic diffuse emission (isotrop_2year_P76_source_v1.txt).

5. We held all of the parameters of the model fixed except for the normalization of the flux of the pulsar under test and isotropic diffuse emission.

We performed this study with both the P7SOURCE_V6 and P7SOURCE_V6MC IRFs, and included the $\phi$ dependence of the $A_{\text {eff }}$ in the exposure calculations. In all cases we measured the pulsars to be almost consistent with having constant fluxes during the first 700 days of the mission. Specifically, for Geminga $P\left(\chi^{2}\right)=0.04$, while for Vela $P\left(\chi^{2}\right)=6.3 \times 10^{-5}$, but adding $2 \%$ error in quadrature brought the $P\left(\chi^{2}\right)$ up to 0.17 . Furthermore, no peak was visible in the Fourier spectra of the fitted fluxes between $12 \mathrm{hr}$ and 700 days. On the other hand, the fitted normalization for the isotropic diffuse component was not consistent with the constant, and in both cases the Fourier analysis showed a significant peak at the 53.4 day orbital precession period (see Figure 87 for results of the Fourier analysis of the Vela flux and associated isotropic normalization). The variability in the normalization of isotropic background is caused by the CR background leakage not having the same $\theta$ dependence as the $\gamma$-ray effective area, so that exposure calculations used to predict the expected counts from the isotropic background template suffer slightly different biases during different phases of the orbital precession period. As we have shown here, this can be handled by leaving the normalization of the isotropic template free, provided that the source dominates the nearby isotropic background.

By comparing results obtained on the Vela pulsar with the P7SOURCE_V6MC IRFs (which include the $\theta$ dependence of the PSF) to results obtained with the P7SOURCE_V6 IRFs (which do not), we estimate that ignoring the $\theta$ dependence of the PSF causes a $\sim 4 \%$ rms variation of the flux of Vela when fitted in 

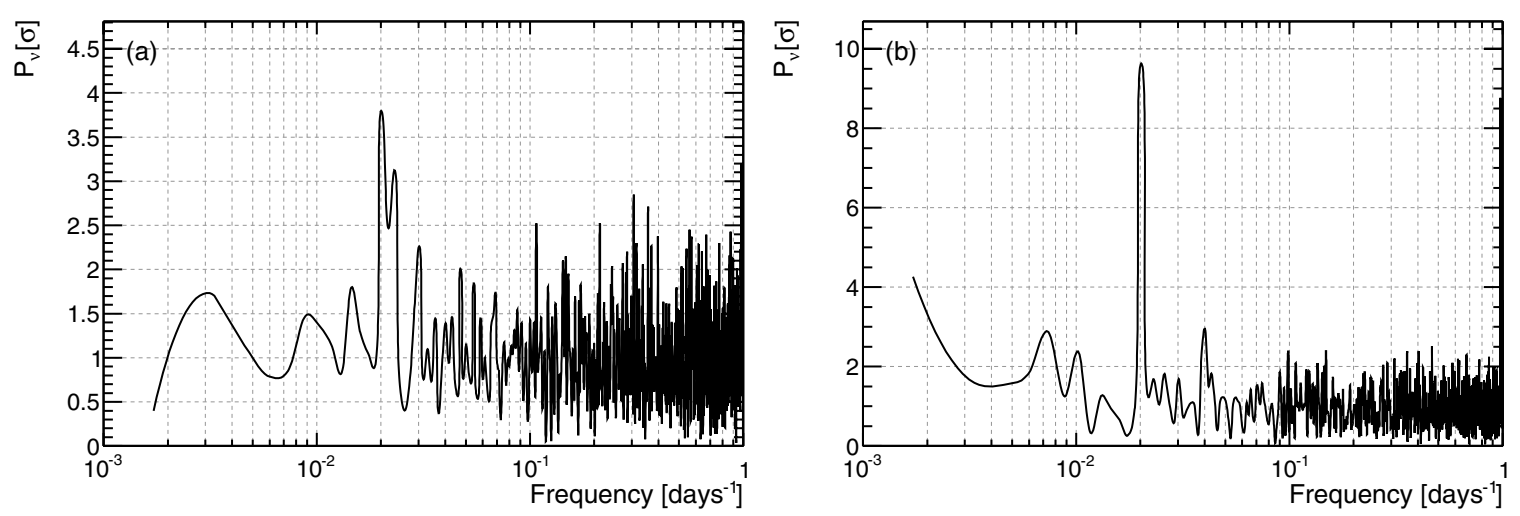

Figure 87. Fourier transforms of the normalized residuals of the Vela integral counts flux (a) and the isotropic normalization factor (b). Note that the figures are normalized and the vertical scale is expressed in units of the statistical uncertainty.

$12 \mathrm{hr}$ time intervals. The effect decreases on longer timescales. Since, as stated in Section 6.1, the PSF depends much more strongly on $\theta$ than on $\phi$, we neglect the $\phi$ dependence of the $\mathrm{PSF}$ as a potential source of instrument-induced variability.

In summary, when the angular dependence of the IRFs is properly accounted for, and time variations of the CR background leakage are absorbed into the normalization of the isotropic component in the likelihood fit, we find the level of instrument-induced variability to be small $(<5 \%)$ for all timescales between $12 \mathrm{hr}$ and 2 years. Since the LAT boresight follows very similar paths across the sky every two orbits, resulting in similar observing profiles, we believe these systematic uncertainties are also applicable at the $3 \mathrm{hr}$ timescale. On the other hand, for observations that do not consist of a complete orbit, and which are therefore more susceptible to biases in the IRFs at specific incidence angles, the $(\sim 10 \%)$ uncertainties in the effective area quoted in Section 5.6.1 are more applicable.

Finally, we note that the slow change in the light yield of the CsI logs of the CAL, and the corresponding change in the energy scale described in Section 7.3.2 can induce slow shifts in observed fluxes by shifting spectra as a function of time. The effect depends on the spectrum of the source under study, as well as on the energy band used, and differs for counts fluxes and energy fluxes, as summarized in Table 23.

\section{SUMMARY}

Since the beginning of science operations in 2008 August, the Fermi-LAT has opened a new window on the $\gamma$-ray sky, providing the science community with an unrivaled set of $\gamma$-ray data. The LAT data set covers the entire sky over the energy range from $\sim 20 \mathrm{MeV}$ to greater than $300 \mathrm{GeV}$ every $3 \mathrm{hr}$.

During these first years of the mission, the LAT Collaboration has studied the on-orbit performance of the LAT, the character of the flight data from the LAT, and the optimization of event selections and IRFs for science. This has led to significant improvements in the event analysis (in particular the Pass 7 version of the event analysis described in Section 3.3) and in the science analysis, and to important clarifications of the systematic uncertainties at each level of the analysis. In addition, the procedures we designed and developed to perform these investigations can be reiterated with minimum modifications whenever a new event reconstruction and classification is adopted.

We have shown that the LAT has performed extremely well: the data are of uniformly high quality, we have lost less than $0.5 \%$ of potential observing time (i.e., when Fermi is outside the SAA) to unplanned outages and instrumental issues. Furthermore, the LAT response has been extremely stable: we are able to use a single set of IRFs for the entire mission to date, with any variations in performance contributing negligibly to systematic uncertainties (Section 2).

We have also confirmed that the LAT data reduction and analysis tools have performed very well. The combination of a configurable hardware trigger, an on-board event filter, and ground-based event reconstruction and analysis have allowed us to reduce the CR contamination from 5 to $10 \mathrm{kHz}$ passing through the instrument to $\sim 1 \mathrm{~Hz}$ in the P7SOURCE event sample (and $\sim 0.1 \mathrm{~Hz}$ in the P7CLEAN event sample, Section 3) while maintaining a peak acceptance of over $2 \mathrm{~m}^{2} \mathrm{sr}$ in the P7SOURCE sample (and $>1.75 \mathrm{~m}^{2} \mathrm{sr}$ in the P7CLEAN sample; Section 5).

We have validated the quality of our MC simulations and found only one significant discrepancy between the MC simulations and the flight data. Specifically, limitations in the pre-launch calibration algorithm of the CAL light asymmetry produced calibration constants that did not match the MC predictions of the spatial resolution performance of the CAL. However, we have quantified the effects of this discrepancy on the $A_{\text {eff }}$ (Section 5.4) and PSF (Section 6.2) and are currently assessing the improvement of the PSF for data that were reprocessed with improved calibration constants.

Pass 7 data have been available for public analysis since 2011 August and provide substantial improvements over Pass 6, primarily due to greatly increased $A_{\text {eff }}$ below $\sim 300 \mathrm{MeV}$ (Section 5.8) and improved modeling of the IRFs. Coupled with improved understanding of the effects of energy dispersion (Section 7), this increase in effective area is opening a new window for analysis of LAT data for energies below $100 \mathrm{MeV}$. Table 25 lists the features of the components included in each of the IRF sets recommended for analysis with both Pass 6 and Pass 7 (Section 7).

In addition to the caveats ${ }^{85}$ and the documentation ${ }^{86}$ accompanying the data and the science analysis software, we provide with this paper a reference document for all currently known systematic issues. Table 26 provides numerical estimates of the residual uncertainties and refers to the sections of this paper where we detail procedures to estimate the systematics uncertainties for many analyses.

Finally, in Section 8, we provide details on the science performance we obtain with the Pass 7 event analysis and IRFs.

\footnotetext{
85 http://fermi.gsfc.nasa.gov/ssc/data/analysis/LAT_caveats.html

86 http://fermi.gsfc.nasa.gov/ssc/data/analysis/documentation/
} 
Table 25

IRF Sets Recommended for Data Analysis, and the Features Included in the Various Components

\begin{tabular}{lcc}
\hline \hline IRF Set & $A_{\text {eff }}$ & PSF \\
\hline P6_V3_TRANSIENT & $f_{\text {live }}, \phi$ & Legacy MC \\
P6_V3_DIFFUSE & $f_{\text {live }}, \phi$ & Legacy MC \\
P6_V3_DATACLEAN & $f_{\text {live }}, \phi$ & Legacy MC \\
\hline P6_V11_DIFFUSE & $f_{\text {live }}, \phi$, in-flight & In-flight \\
\hline P7TRANSIENT_V6 & $f_{\text {live }}, \phi$ & In-flight \\
P7SOURCE_V6 & $f_{\text {live }}, \phi$ & In-flight \\
P7CLEAN_V6 & $f_{\text {live }}, \phi$ & In-flight \\
P7ULTRACLEAN_V6 & $f_{\text {live }}, \phi$ & In-flight \\
\hline P7SOURCE_V6MC & $f_{\text {live }}, \phi$ & MC \\
P7CLEAN_V6MC & $f_{\text {live }}, \phi$ & MC \\
\hline
\end{tabular}

Notes. For $A_{\text {eff }}, f_{\text {live }}$ and $\phi$ refer to the live time fraction and $\phi$ dependence, respectively (Sections 5.2.2 and 5.2.3), and "in-flight" refers to flight-based corrections (Section 5.4). For the PSF "Legacy MC" refers to an early version of the parameterization fit to allGamma samples (Section 6.1.2), "in-flight" refers to the flight-based PSF (Section 6.2), and "MC" refers to the more recent parameterization fit to allGamma samples (Section 6.1.1). Finally, we have used the same parameterization of the energy dispersion for all of the IRF sets (Section 7.1.2).

In particular, we provide estimates of the source detection sensitivity threshold (Section 8.1), the source localization performance (Section 8.2), the expected precision and accuracy of measurements of fluxes and spectral indices (Section 8.3), and the precision and accuracy of variability measurements (Section 8.4).

As stated in Section 1, the LAT team will continue to make both major improvements and minor refinements to many aspects of the event reconstruction, analysis, and to the associated IRFs. We will continue to keep the $\gamma$-ray astronomy community informed of the state of the art of our understanding of the LAT and issues relating to analyzing LAT data.

The Fermi-LAT Collaboration acknowledges generous ongoing support from a number of agencies and institutes that have supported both the development and the operation of the LAT as well as scientific data analysis. These include the National Aeronautics and Space Administration and the Department of Energy in the United States, the Commissariat à l'Energie Atomique and the Centre National de la Recherche Scientifique/Institut National de Physique Nucléaire et de Physique des Particules in France, the Agenzia Spaziale Italiana and the Istituto Nazionale di Fisica Nucleare in Italy, the Ministry of Education, Culture, Sports, Science and Technology (MEXT), High Energy Accelerator Research Organization (KEK) and Japan Aerospace Ex-
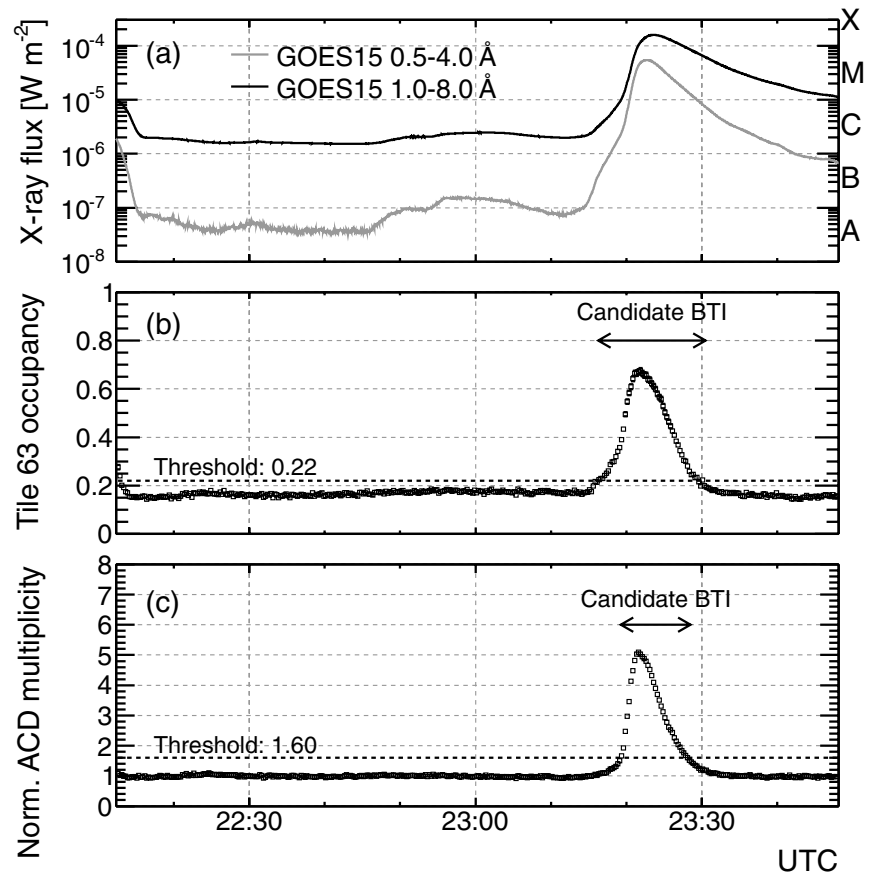

Figure 88. (a) X-ray flux for the X1.5 SF on 2011 March 9 measured by the GOES-15 satellite in the energy bands $0.5-4.0 \AA(3.1-24.8 \mathrm{keV})$ and $1.0-8.0 \AA$ $(1.5-12.4 \mathrm{keV})$. The latter is customarily used to classify SFs based on their $\mathrm{X}$-ray radiance, as indicated by the letters $\mathrm{A}, \mathrm{B}, \mathrm{C}, \mathrm{M}$, and $\mathrm{X}$ on the right. The effect on two of the basic ACD quantities (see the text for more details) is clearly visible in panels (b) and (c).

ploration Agency (JAXA) in Japan, and the K. A. Wallenberg Foundation, the Swedish Research Council and the Swedish National Space Board in Sweden.

Additional support for science analysis during the operations phase is gratefully acknowledged from the Istituto Nazionale di Astrofisica in Italy and the Centre National d'Études Spatiales in France.

The Parkes radio telescope is part of the Australia Telescope, which is funded by the Commonwealth Government for operation as a National Facility managed by CSIRO. We thank our colleagues for their assistance with the radio timing observations.

\section{APPENDIX A}

\section{SOLAR FLARES AND BAD TIME INTERVALS}

With the beginning of the solar activity connected with Cycle 24 , in early 2009, it became clear that SFs can cause significant $\mathrm{X}$-ray pileup in the ACD, clearly visible in many low-level ACD

Table 26

Rough Estimates of the Magnitude of the Effects of Various Sources of Systematic Errors for Commonly Measured $\gamma$-Ray Source Properties

\begin{tabular}{lcccc}
\hline \hline Quantity & \multicolumn{1}{c}{$A_{\text {eff }}$} & PSF & Energy \\
\cline { 3 - 5 } & & & Dispersion & Scale \\
\hline$F_{25}$ & $\sim 8 \%($ Section 5.7$)$ & $\sim 8 \%($ Section 6.5$)$ & $\sim 3 \%($ Section 7.4$)$ & $+13 \%-5 \%($ Section 7.4$)$ \\
$S_{25}$ & $\sim 10 \%($ Section 5.7$)$ & $\sim 6 \%($ Section 6.5$)$ & $\sim 2 \%($ Section 7.4$)$ & $+4 \%-2 \%($ Section 7.4$)$ \\
$\Gamma$ & $\sim 0.09$ (Section 5.7$)$ & $\sim 0.07($ Section 6.5$)$ & $\sim 0.04($ Section 7.4$)$ & $\ldots$ \\
Variability & $\sim 3 \%($ Section 5.6$)$ & $\sim 3 \%($ Section 6.5$)$ & $\ldots$ & $\ldots$ \\
Localization & $\ldots$ & $\sim 0.005(\text { Section } 8.2)^{\mathrm{a}}$ & $\ldots$ & $\ldots$ \\
\hline
\end{tabular}

Notes. We also provide references to the relevant sections with more details.

a See Nolan et al. (2012) for a discussion of the systematic uncertainties on source localization. 


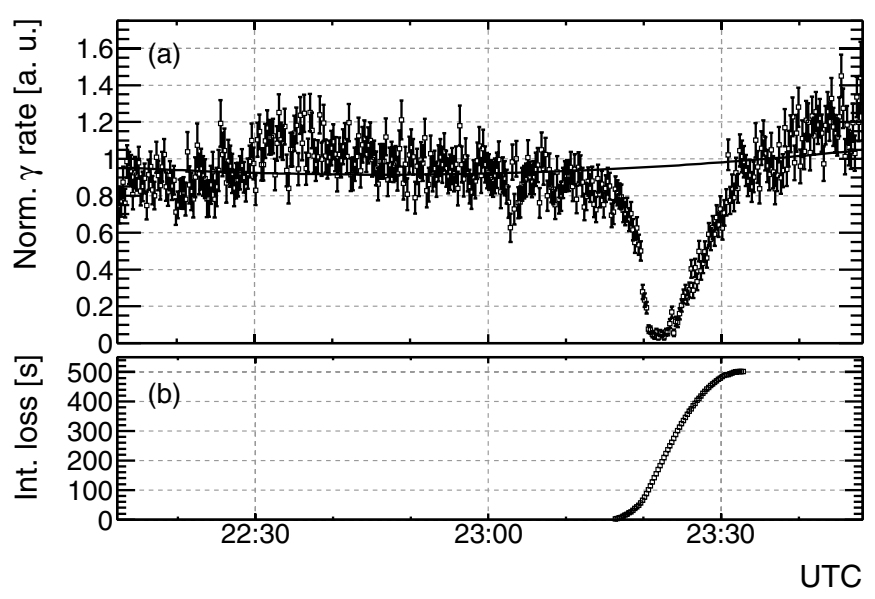

Figure 89. (a) Normalized rate of P7TRANSIENT events for the same time interval shown in Figure 88 and (b) integrated time loss over the candidate BTI in Figure 88(b). Each data point corresponds to a $15 \mathrm{~s}$ time bin. The solid line is a second-order polynomial fit to the normalized rate outside the candidate BTI and it is used to calculate the integrated time loss, as defined in Equation (A1).

quantities (see Figure 88). The on-board ACD veto electronics (see Table 1) are fast enough that this is not causing any change in the efficiency of the trigger and/or that of the GAMMA filter (i.e., there is essentially no loss of $\gamma$ rays onboard). However, the effects of the pileup are potentially much more severe at the level of the event selection on the ground-where the slow signals are used - as the additional activity in the ACD can cause $\gamma$ rays to be misclassified as background.

The basic phenomenology is somewhat similar to the ghostinduced loss of efficiency introduced in Section 2.1.4 and discussed in detail throughout the paper, the main difference being that during the most intense SFs, the effect can be large enough to make the LAT essentially blind to $\gamma$ rays. From the point of view of the data analysis, the most relevant implication is that there are time intervals in which the standard IRFs do not accurately represent the detector and therefore the results of the likelihood analysis are potentially unreliable. While the LAT Collaboration is considering possible modifications to the event reconstruction and selection aimed at mitigating the problem, these bad time intervals (BTIs) are being systematically identified and flagged.

Operationally, a BTI is characterized by a suppression of the rate of events in the standard $\gamma$-ray classes. The $\gamma$-ray rates intrinsically feature large orbital variations depending on both the geomagnetic environment (through the background leakage and the ghost effect) and the rocking angle of the observatory (through the change of the arc length of the Earth limb in the FoV). These variations can be parameterized to an accuracy of 20\%-30\% and in fact are accounted for (at a similar level of accuracy) in event rates normalized to predicted values that are routinely accumulated in $15 \mathrm{~s}$ time bins for data monitoring purposes (see, e.g., Figure 89). Still, the residual variations (especially during rocking maneuvers or non-standard pointings) make the normalized rates not directly suitable for identifying the BTIs. We use a two-step procedure instead, in which

1. within each orbit we search for evidence of significant $\mathrm{X}$-ray pileup activity in the ACD in order to define a list of candidate BTIs (see Figure 88) and

2. we search for a temporally coincident decrease in the transient $\gamma$-ray rate within each of those intervals (see Figure 89).

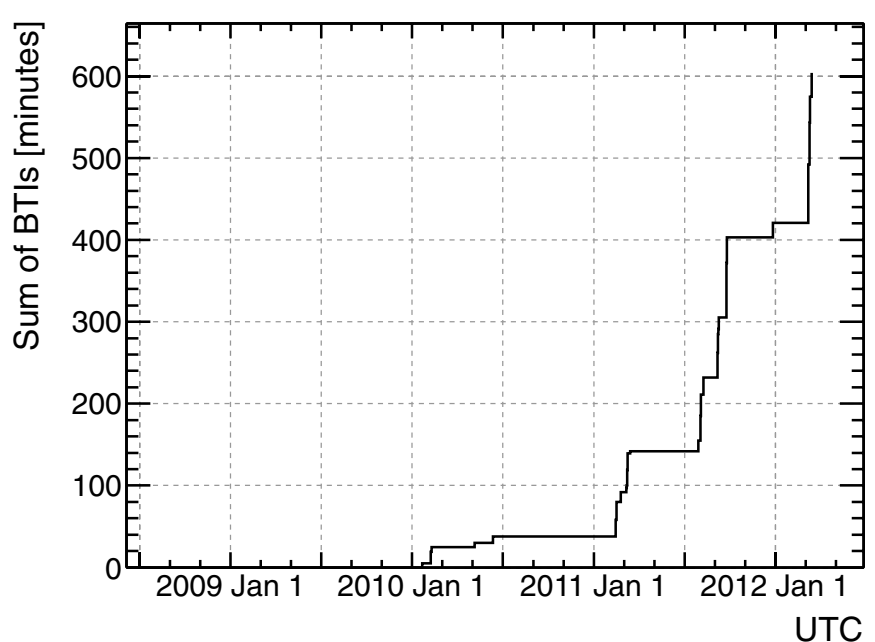

Figure 90. Time history of the sum of BTIs induced by SFs. The sum of time intervals marked as $B A D$ as of 2012 March is $\sim 600$ minutes (i.e., $\sim 10 \mathrm{hr}$ ).

As shown in Figure 88, X-ray pileup induced by bright flares is typically visible both in the normalized ACD hit multiplicity and in the single tile hit occupancy-particularly in tile 63, which is the largest tile on the $+x$ side of the observatory (i.e., the side facing the Sun). Though the correlation with the $\mathrm{X}$-ray radiance measured by GOES is far from perfect (with a zero-suppression threshold of $\sim 100 \mathrm{keV}$ the ACD is sensitive to somewhat higher energies than GOES), both quantities are good proxies for the increase in the X-ray flux. Figure 89(a) shows the corresponding large suppression of the normalized P7TRANSIENT rate for the same SF. As shown in Figure 89(b), by fitting this normalized rate outside the candidate BTI it is also possible to define an integrated time loss $T_{\text {loss }}$ corresponding to a particular candidate BTI

$$
T_{\text {loss }}=\sum_{\text {BTI }}\left(f_{i}-r_{i}\right) \Delta t
$$

(where $f_{i}$ and $r_{i}$ are the fitted and measured normalized rates in the $i$ th time bin, respectively, and $\Delta t$ is the bin width, i.e., $15 \mathrm{~s}$ ). Roughly speaking, if $T_{\text {loss }}$ is larger than a few minutes we will mark the relevant time interval as bad.

All LAT $\gamma$-ray data automatically have the DATA_QUAL field in the spacecraft pointing and live time history file (spacecraft file $)^{87}$ set to 1 by the data processing system and are immediately exported to the FSSC where they become publicly available. The potential for BTIs is reviewed only after the fact. This is because such a high percentage of the LAT data is good and making them publicly available as quickly as possible is a priority.

For each flare, the entire information available is reviewed manually and if there is a significant loss of efficiency the corresponding time period is marked as $B A D$ (specifically, the DATA_QUAL field is set to -1) and a new spacecraft file is generated with the appropriate data quality flag in the corresponding $30 \mathrm{~s}$ time bins. This new file is then exported to the FSSC and supersedes the original file. As of 2012 March, the sum of time intervals marked as $B A D$ is $\sim 10 \mathrm{hr}$ (Figure 90).

\footnotetext{
87 http://fermi.gsfc.nasa.gov/ssc/data/analysis/documentation/Cicerone/ Cicerone_Data/LAT_Data_Columns.html\#SpacecraftFile
} 


\section{APPENDIX B}

\section{LIST OF ACRONYMS AND ABBREVIATIONS}

\begin{tabular}{|c|c|}
\hline 2FGL & Fermi-LAT second source catalog \\
\hline $\mathrm{ACD}$ & LAT anticoincidence detector subsystem \\
\hline AGN & Active galactic nucleus \\
\hline BTI & Bad time interval \\
\hline BSPR & Blind search pattern recognition \\
\hline CAL & LAT imaging calorimeter subsystem \\
\hline $\mathrm{CNO}$ & Carbon, nitrogen, oxygen (CR species) \\
\hline $\mathrm{CPF}$ & Charged particle in the field of view analysis \\
\hline CR & Cosmic ray \\
\hline CSPR & Calorimeter-seeded pattern recognition \\
\hline $\mathrm{CT}$ & Classification tree \\
\hline $\mathrm{CU}$ & LAT Calibration Unit \\
\hline EGRET & Energetic Gamma-Ray Experiment Telescope \\
\hline Fermi & Fermi Gamma-ray Space Telescope \\
\hline FoV & Field of view \\
\hline FSSC & Fermi Science Support Center \\
\hline GRB & Gamma-ray burst \\
\hline GSI & Gesellschaft für SchwerIonenforschung \\
\hline IRF & Instrument response function \\
\hline ISOC & Instrument Science Operations Center \\
\hline LAT & Fermi Large Area Telescope \\
\hline LH & Maximum likelihood (energy estimation algorithm) \\
\hline $\mathrm{MC}$ & Monte Carlo \\
\hline MET & Mission elapsed time \\
\hline MIP & Minimum ionizing particle \\
\hline $\mathrm{PC}$ & Parametric correction (energy estimation algorithm) \\
\hline p.e. & photoelectron \\
\hline PMT & Photomultiplier tube \\
\hline PS & CERN Proton Synchrotron \\
\hline PSF & Point-spread function \\
\hline ROI & Region of interest \\
\hline SAA & South Atlantic Anomaly \\
\hline SF & Solar flare \\
\hline SP & Shower profile (energy estimation algorithm) \\
\hline SSD & Silicon strip detector \\
\hline SPS & CERN Super Proton Synchrotron \\
\hline TKR & LAT tracker/converter subsystem \\
\hline
\end{tabular}

Section 3.6.2

Section 2.1

Section 3.6.2

Section A

Section 3.2.2

Section 2.1

Section 5

Section 3.3.5

Section 1

Section 3.2.2

Section 3.3.2

Section 7.3.1

Section 2.1.3

Section 1

Section 1

Section 1

Section 3.4.1

Section 7.3.1

Section 1

Section 1

Section 1

Section 3.2.1

Section 1

Section 6

Section 2.1.1

Section 3.2.1

Section 2.1.3

Section 2.1.3

Section 7.3.1

Section 2

Section 6

Section 2.2

Section 2.1.4

Section 3.2.1

Section 2.1

Section 7.3.1

Section 2.1

\section{APPENDIX C}

NOTATION

$A_{\mathrm{eff}}$
$B, B_{\mathrm{a}}, B_{\mathrm{idx}}$
$B, B_{68}, B_{r}$
$b$
$b$
$(l, b)$
$C_{68}$
$C_{i}$

Effective area

Bracketing functions for $A_{\text {eff }}$

Bracketing functions for the PSF

Bias in the energy scale

Distribution of CR background events mischaracterized as $\gamma$ rays

Galactic coordinates

$68 \%$ containment radius of the PSF

Actual containment at a given nominal containment radius
Section 1

Section 5.7.1

Section 6.5.1

Section 7.4

Section 4.6

Section 3.3.3

Section 6.5.3 


\begin{tabular}{|c|c|}
\hline$c_{i}$ & Generic fitting constants \\
\hline$D$ & Energy dispersion parameterization \\
\hline$d_{i}$ & Normalized deviations \\
\hline$E$ & Energy \\
\hline$F_{25}$ & Integral counts flux between $100 \mathrm{MeV}$ and $100 \mathrm{GeV}$ \\
\hline$F_{l}$ & Live time fraction \\
\hline$f_{\text {core }}$ & Fraction of counts in the "core" King function for the PSF \\
\hline$K$ & King function (used to model the PSF) \\
\hline$k$ & $A_{\text {eff }}$ bracketing smoothing constant \\
\hline$L$ & McIlwain L \\
\hline$L_{\text {ext }}$ & log likelihood for fit as an extended source \\
\hline$L_{\mathrm{pt}}$ & log likelihood for fit as a point source \\
\hline$M$ & Predicted counts distribution of $\gamma$ rays. \\
\hline$N_{0}$ & Flux prefactor \\
\hline$N_{\text {gen }}$ & Number of events generated (when making IRFs) \\
\hline$n$ & Number of observed counts \\
\hline$\tilde{n}$ & Expected counts \\
\hline$P$ & Point-spread function parameterization \\
\hline $\bar{P}$ & PSF averaged over the observing profile \\
\hline$P_{\text {all }}$ & Combined estimator that the event is a $\gamma$ ray \\
\hline$P_{\mathrm{CAL}}$ & Estimator that the event is a $\gamma$ ray, based on the CAL topology analysis \\
\hline$P_{\text {core }}$ & Estimator of the quality of direction measurement \\
\hline$P_{\mathrm{CPF}}$ & Estimator that the event is a $\gamma$ ray, based on the CPF analysis \\
\hline$P_{\mathrm{E}}$ & Estimate of the quality of energy measurement \\
\hline$P_{\mathrm{TKR}}$ & Estimator that the event is a $\gamma$ ray, based on the TKR topology analysis \\
\hline$\hat{p}$ & Incident direction in celestial reference frame \\
\hline$R$ & Rando function, used to model the energy dispersion \\
\hline$R$ & Ratio of efficiency for flight data to MC simulated data \\
\hline$R_{68}$ & $68 \%$ containment radius of the PSF (also $R_{95}$ ) \\
\hline$r$ & Ratio of size of signal region to background region \\
\hline$r$ & Ratio of $95 \%$ to $68 \%$ containment radii of the PSF \\
\hline$r_{i}^{n}$ & Normalized residuals \\
\hline$r_{i}^{f}$ & Fractional residuals \\
\hline$S$ & Source distribution of $\gamma$ rays \\
\hline$S_{25}$ & Integral energy flux between $100 \mathrm{MeV}$ and $100 \mathrm{GeV}$ \\
\hline$S_{D}$ & Energy dispersion scaling function \\
\hline$S_{P}$ & PSF scaling function \\
\hline$s$ & Light yield scaling function \\
\hline $\mathrm{TS}_{\mathrm{ext}}$ & Test statistic for source extension \\
\hline$t_{\mathrm{obs}}$ & Observing time \\
\hline$\hat{v}$ & Incident direction in the LAT frame \\
\hline$x$ & Scaled angular deviation \\
\hline$x$ & Scaled energy redistribution \\
\hline$\tilde{x}$ & Rando function break point \\
\hline$\alpha$ & Spectral index in the "log-parabola" model \\
\hline$\beta$ & Spectral curvature parameter in the "log-parabola" model \\
\hline$\beta$ & PSF energy scaling index \\
\hline$\Gamma$ & Spectral index \\
\hline$\gamma$ & King function "tail” parameter \\
\hline$\gamma$ & Rando function "tail" parameter \\
\hline
\end{tabular}

Section 1

Section 5.6.2

Section 1

Section 5.7.1

Section 5.2.2

Section 6.1.1

Section 6.1.1

Section 5.7.1

Section 2.5.1

Section 6.5.2

Section 6.5.2

Section 1

Section 5.7.1

Section 5.1

Section 5.1

Section 5.6.3

Section 1

Section 6.1

Section 4

Section 3.3.7

Section 3.3.3

Section 3.3.5

Section 3.3.2

Section 3.3.6

Section 1

Section 7.1.2

Section 5.3

Section 6.2.3

Section 5.3.1

Section 6.5.1

Section 7.5

Section 7.5

Section 1

Section 5.7.1

Section 7.1.1

Section 1

Section 7.3.2

Section 6.5.2

Section 1

Section 1

Section 6.1.1

Section 7.1.1

Section 7.1.2

Section 5.7.1

Section 5.7.1

Section 6.1.1

Section 5.7.1

Section 6.1.1

Section 7.1.2 


$\epsilon$
$\eta_{\text {data }}, \eta_{\mathrm{mc}}$
$(\theta, \phi)$
$\left(\theta_{z}, \phi_{z}\right)$
$\hat{\theta}, \hat{\phi}$
$\xi$
$\sigma$
$\sigma$
$\tau_{n}$

Relative uncertainty in $A_{\text {eff }}$

Efficiencies for flight data and MC simulated data

Polar angle and azimuth of the incident direction in the LAT frame

Zenith angle and Earth azimuth angle

Unit vector along local $\theta, \phi$ directions

Folded $\phi$

King function width parameter

Rando function width parameter

Correlation metric
Section 5.7.1

Section 5.3.1

Section 2.1

Section 2.2

Section 6.4

Section 5.2.3

Section 6.1.1

Section 7.1.2

Section 5.6.2

\section{REFERENCES}

Abdo, A. A., Ackermann, M., Agudo, I., et al. 2010a, ApJ, 716, 30 Abdo, A. A., Ackermann, M., Ajello, M., et al. 2009a, Astropart. Phys., 32, 193 Abdo, A. A., Ackermann, M., Ajello, M., et al. 2009b, Phys. Rev. Lett., 102, 181101

Abdo, A. A., Ackermann, M., Ajello, M., et al. 2009c, Phys. Rev. D, 80, 122004

Abdo, A. A., Ackermann, M., Ajello, M., et al. 2010b, ApJS, 188, 405

Abdo, A. A., Ackermann, M., Ajello, M., et al. 2010c, ApJ, 713, 154

Abdo, A. A., Ackermann, M., Ajello, M., et al. 2010d, ApJ, 708, 1254

Abdo, A. A., Ackermann, M., Ajello, M., et al. 2010e, Phys. Rev. Lett., 104, 101101

Abdo, A. A., Ackermann, M., Ajello, M., et al. 2010f, Phys. Rev. Lett., 104, 091302

Abdo, A. A., Ackermann, M., Ajello, M., et al. 2010g, ApJ, 712, 459

Abdo, A. A., Ackermann, M., Ajello, M., et al. 2010h, ApJ, 720, 272

Abdo, A. A., Ackermann, M., Asano, K., et al. 2009d, ApJ, 707, 580

Abdo, A. A., Ackermann, M., Atwood, W. B., et al. 2009e, ApJ, 696, 1084

Ackermann, M., Ajello, M., Allafort, A., et al. 2012a, Astropart. Phys., 35, 346

Ackermann, M., Ajello, M., Allafort, A., et al. 2012b, Phys. Rev. Lett., 108, 011103

Ackermann, M., Ajello, M., Allafort, A., et al. 2012c, ApJ, 745, 144

Ackermann, M., Ajello, M., Atwood, W. B., et al. 2010, Phys. Rev. D, 82, 092004

Ackermann, M., Ajello, M., Atwood, W. B., et al. 2012d, ApJ, 750, 3

Ackermann, M., et al. 2012e, ApJ, submitted

Agostinelli, S., Allison, J., Amako, K., et al. 2003, Nucl. Instrum. Methods Phys. Res. A, 506, 250

Arnaud, K. A. 1996, in ASP Conf. Ser. 101, Astronomical Data Analysis Software and Systems V, ed. G. H. Jacoby \& J. Barnes (San Francisco, CA: ASP), 17

Atwood, W. B., Abdo, A. A., Ackermann, M., et al. 2009, ApJ, 697, 1071

Atwood, W. B., Bagagli, R., Baldini, L., et al. 2007, Astropart. Phys., 28, 422

Baldini, L., Barbiellini, G., Bellazzini, R., et al. 2007, in AIP Conf. Proc. 921, The First GLAST Symposium, ed. S. Ritz, P. Michelson, \& C. A. Meegan (Melville, NY: AIP), 190

Breiman, L. 1996, Mach. Learn., 24, 123
Breiman, L., Friedman, J., Olshen, R., \& Stone, C. 1984, Classification and Regression Trees (New York: Chapman \& Hall), 358

D’Agostini, G. 1995, Nucl. Instrum. Methods Phys. Res. A, 362, 487

Efron, B., \& Tibshirani, R. 1993, An Introduction to the Bootstrap (Boca Raton, FL: Chapman \& Hall)

Esposito, J. A., Bertsch, D. L., Chen, A. W., et al. 1999, ApJS, 123, 203

Finlay, C. C., Maus, S., Beggan, C. D., et al. 2010, Geophys. J. Int., 183, 1216

Fruhwirth, R. 1987, Nucl. Instrum. Methods Phys. Res. A, 262, 444

Grove, J. E., \& Johnson, N. 2010, Proc. SPIE, 7732, 77320J

Hobbs, G. B., Edwards, R. T., \& Manchester, R. N. 2006, MNRAS, 369, 655

Kalman, R. E. 1960, J. Basic Engineering D, 82, 35

King, I. 1962, AJ, 67, 471

Kirsch, M. G. F., Altieri, B., Chen, B., et al. 2004, Proc. SPIE, 5488, 103

Lande, J., Ackermann, M., Allafort, A., et al. 2012, ApJ, 756, 5

Lee, H., Kashyap, V. L., van Dyk, D. A., et al. 2011, ApJ, 731, 126

Lott, B., Piron, F., Blank, B., et al. 2006, Nucl. Instrum. Methods Phys. Res. A, 560,395

Mattox, J. R., Bertsch, D. L., Chiang, J., et al. 1996, ApJ, 461, 396

Mizuno, T., Kamae, T., Godfrey, G., et al. 2004, ApJ, 614, 1113

Moiseev, A. A., Hartman, R. C., Ormes, J. F., et al. 2007, Astropart. Phys., 27, 339

Moskalenko, I. V., \& Porter, T. A. 2007, ApJ, 670, 1467

Nolan, P. L., Abdo, A. A., Ackermann, M., et al. 2012, ApJS, 199, 31

Pelassa, V., Preece, R., Piron, F., et al. 2010, in Proc. 2009 Fermi Symp. eConf. Proc. C091122

Rando, R., \& The Fermi LAT Collaboration. 2009, in Proc. ICRC 31

Read, A. M., Rosen, S. R., Saxton, R. D., \& Ramirez, J. 2011, A\&A, 534, A34

Rochester, L., Usher, T., Johnson, R. P., \& Atwood, B. 2010, in Proc. 2009 Fermi Symp. eConf. Proc. C091122

Smart, D. F., \& Shea, M. A. 2005, Adv. Space Res., 36, 2012

Smith, D. A., Guillemot, L., Camilo, F., et al. 2008, A\&A, 492, 923

Student 1908, Biometrika, 6, 1

Walt, M. 2005, Introduction to Geomagnetically Trapped Radiation, Cambridge Atmospheric and Space Science Series (Cambridge: Cambridge Univ. Press)

Weltevrede, P., Johnston, S., Manchester, R. N., et al. 2010, PASA, 27, 64 